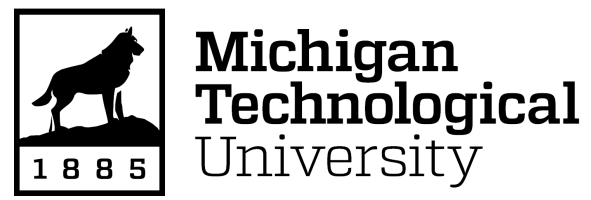

Michigan Technological University Digital Commons @ Michigan Tech

Dissertations, Master's Theses and Master's Reports

2018

Meniscus Modeling and Emission Studies of an Ionic Liquid Ferrofluid Electrospray Source Emitting from a Magneto-Electric Instability

Brandon Jackson

Michigan Technological University, bajackso@mtu.edu

Copyright 2018 Brandon Jackson

Recommended Citation

Jackson, Brandon, "Meniscus Modeling and Emission Studies of an Ionic Liquid Ferrofluid Electrospray Source Emitting from a Magneto-Electric Instability", Open Access Dissertation, Michigan Technological University, 2018.

https://doi.org/10.37099/mtu.dc.etdr/587

Follow this and additional works at: https://digitalcommons.mtu.edu/etdr

Part of the Other Mechanical Engineering Commons, and the Propulsion and Power Commons 


\title{
MENISCUS MODELING AND EMISSION STUDIES OF AN IONIC LIQUID FERROFLUID ELECTROSPRAY SOURCE EMITTING FROM A MAGNETO- ELECTRIC INSTABILITY
}

By

Brandon A. Jackson

\begin{abstract}
A DISSERTATION
Submitted in partial fulfillment of the requirements for the degree of DOCTOR OF PHILOSOPHY

In Mechanical Engineering_Engineering Mechanics
\end{abstract}

MICHIGAN TECHNOLOGICAL UNIVERSITY

2018

C2018 Brandon A. Jackson 

This dissertation has been approved in partial fulfillment of the requirements for the Degree of DOCTOR OF PHILOSOPHY in Mechanical Engineering - Engineering Mechanics.

Department of Mechanical Engineering - Engineering Mechanics

Dissertation Advisor:

Dr. Lyon B. King

Committee Member:

Dr. Jeffrey S. Allen

Committee Member:

Dr. Chang K. Choi

Committee Member:

Dr. Durdu O. Guney

Department Chair:

Dr. William W. Predebon 

“In nature's infinite book of secrecy

A little I can read."

William Shakespeare

(Antony and Cleopatra) 



\section{Contents}

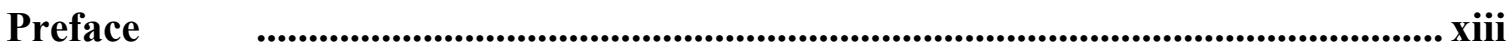

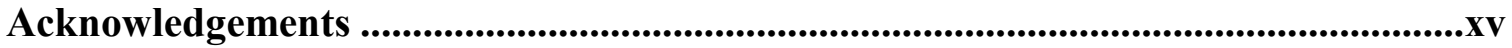

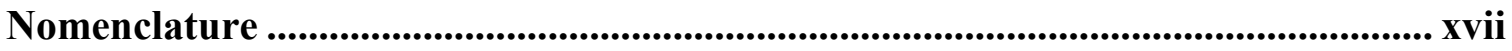

Abstract

Chapter 1 Electric Spacecraft Propulsion ..............................................................1

1.1 Application of Electrospray Propulsion ................................................

1.2 Electrospray Propulsion - Past and Present ...............................................5

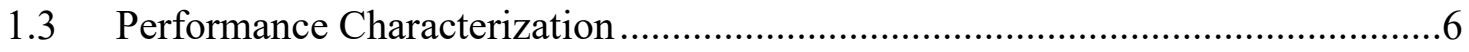

1.4 Ionic Liquid Ferrofluid Electrospray Thrusters ...............................................

Chapter 2 Overview of Electrospray and Electrohydrodynamics .......................13

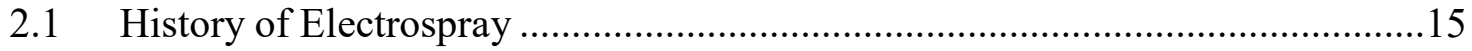

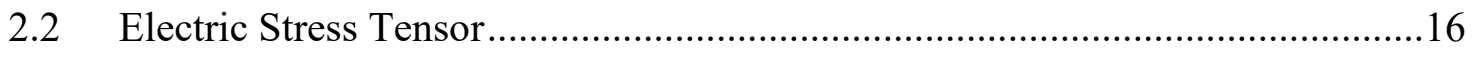

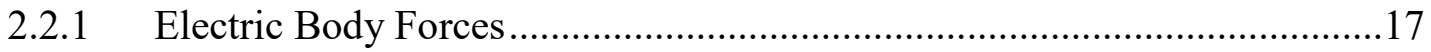




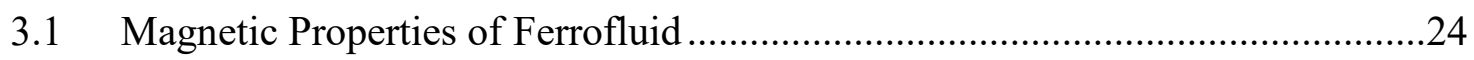

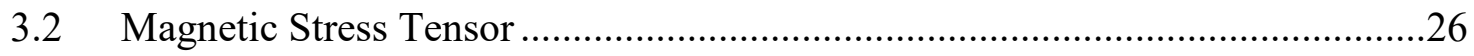

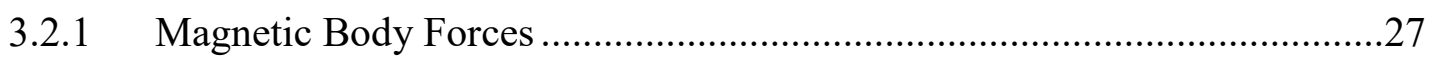

3.2.2 Ferrofluid Interface Stress Components …........................................28

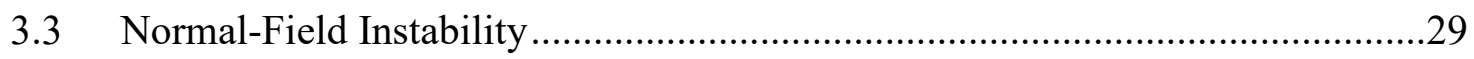

Chapter 4 Modeling Interface Deformation and Spray Onset of a Ferrofluid Meniscus.................................................................................................................33

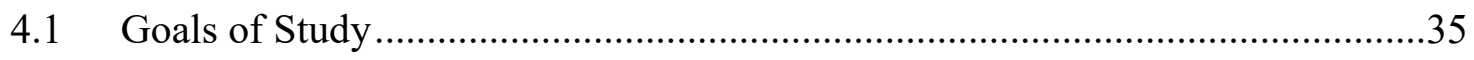

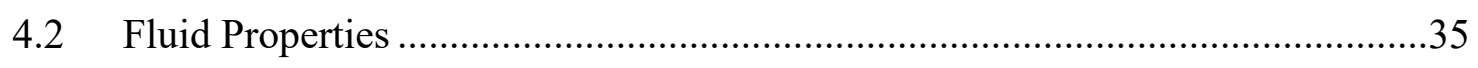

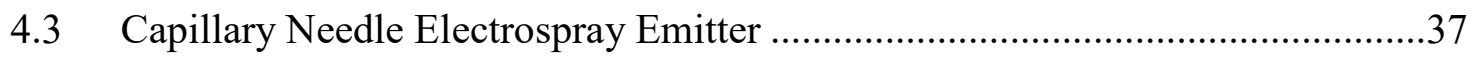

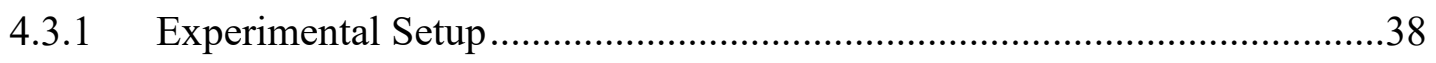

4.3.2 Computational Approach ............................................................. 39

4.3.3 Simulation Results and Comparison with Experiment ............................45

4.4 Predictive Model of Electromagnetic Sessile Droplet Deformation ..................48

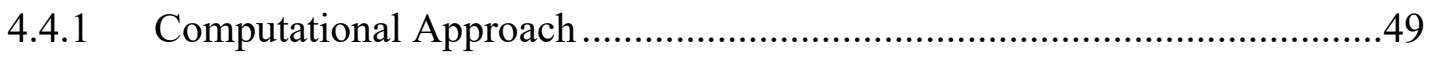

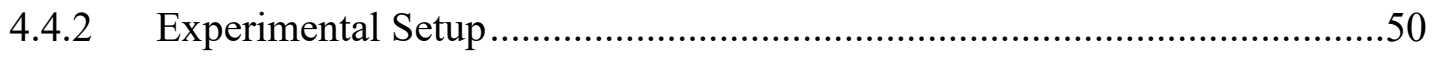

4.4.3 Simulation Results and Comparison with Experiment .............................55 
4.5 Conclusions

\section{Chapter 5 Emission Studies of an ILFF Electrospray via the Normal-Field}

Instability ...........................................................................................61

5.1 Goals of Study

5.2 Equipment and Testing Methodology.

5.2.1 Electrospray Source

5.2.2 High Voltage Power Supply .64

5.2.3 Segmented Faraday Probe and $\mu \mathrm{A}$ Current Measurement. 66

5.2.4 Vacuum Facility. .69

5.2.5 Testing Methodology and Telemetry Phases..... .70

5.3 Influence of Peak Sharpness on Emission Behavior. .73

5.4 Long Duration Emission of a Single Emitter.

5.4.1 Mass Flow Rate. .85

5.4.2 Derived Propulsion Performance Parameters . .91

5.5 Source Sensitivity and Emission Site Dynamics.......

5.6 Conclusions 100

\section{Chapter 6 Angular Emission Profile of an ILFF Electrospray Operating via the} Normal-Field Instability . .103

6.1 Goals of Study 105 
6.2 Overview of Divergence Measuring Techniques 106

6.3 In Situ Measurement of Angular Current Distribution Methodology and Results.......

6.4 Angular Power Efficiency Factor.

6.4.1 Dependence on Beam Polarity

6.4.2 Dependence on Emission Current and Mass Flow Rate

6.5 Conclusions

7.1 Goals of Study 126

7.2 Kelvin Force Modeling and Results......

7.2.1 Modeling Approach

7.2.2 Kelvin Force Modeling Results ....

7.3 Lorentz Force Modeling and Results

7.4 Conclusions

\section{Chapter 8 Conclusions}

8.1 Electric Propulsion Viability. 142

8.2 Electrically and Magnetically Stressed Fluid Surface Modeling Techniques...144 
A1. Surface Stress Components

A2. Normal Stress Component ...................................................................... 148

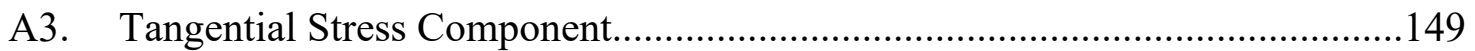

Appendix B Magnetic Stress Component Derivations.........................................151

B1. Surface Stress Components ..................................................................... 151

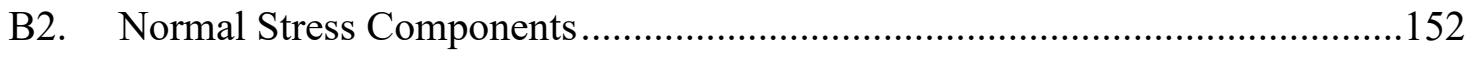

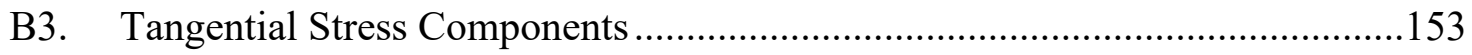

Appendix C Methodology of Processing Time-lapse Imagery to Estimate Mass

Flow Rate ....................................................................................................155

Appendix D Initial Angular Divergence Studies via Rotating Faraday Collector 159

Appendix E Angular Power Efficiency Factor and Correlations Calculation

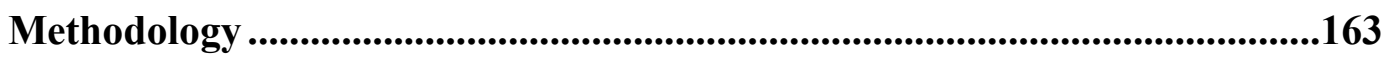

E1. Angular Power Efficiency Factor............................................................ 163

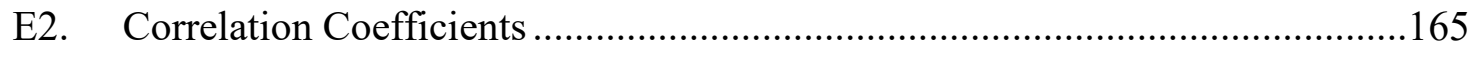

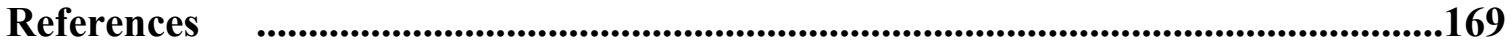





\section{Preface}

A majority of Chapter 4 titled "Modeling Interface Deformation and Spray Onset of a Ferrofluid Meniscus" appears in the following publication:

Jackson, B. A., Terhune, K. J., and King, L. B. "Ionic liquid ferrofluid

interface deformation and spray onset under electric and magnetic stresses," Physics of Fluids Vol. 29, No. 6, 2017, p. 064105. doi: $10.1063 / 1.4985141$

The copyright is retained by Brandon A. Jackson. In this publication Kurt J. Terhune performed the experiential onset studies for the needle emitter utilized for the model validation. Material presented in this dissertation resulting from his efforts are credited where they appear. Lyon B. King motivated and guided the research, assisted in the preparation of the manuscript.

Throughout this report the term "traditional electrospray" will be included regularly. This phrase will reserved to describe electrospray achieved from internally and externally wetted needles as well as porous emitter arrays. 



\section{Acknowledgements}

In finishing this dissertation, I am grateful to many who have helped and supported me during my endeavors at Michigan Technological University.

- First, my advisor Dr. Lyon (Brad). King. Thank you for taking a chance on me when I first contacted you expressing interest in working in your lab. I am thankful for you for giving me the opportunity to pursue a Ph.D. Your guidance as a mentorship were instrumental in my successful completion of this work and professional development.

- My gratitude also goes out to Dr. Jeff Allen. Thank you for opening your office to my frequent surprise visits and providing advice and support as I completed this research.

- Next, I would like to thank my parents. Since I was young, you have instilled the importance of education in my life and worked hard to provide me with the opportunities to pursue it. You have always had confidence in and believed in me, even though I can't say I have always felt the same about myself.

- Finally, I would like to thank my girlfriend Megan who has been supportive and patient with me as I pursued this degree. 



\section{Nomenclature}

$\begin{array}{ll}\vec{a}_{m} & \text { Lorentz Acceleration }\left(\mathrm{m} / \mathrm{s}^{2}\right) \\ \vec{B} & \text { Magnetic Field Vector }(\mathrm{T}) \\ B_{e} & \text { Electric Bond Number } \\ B_{m} & \text { Magnetic Bond Number } \\ \vec{D} & \text { Electric Displacement Field }\left(\mathrm{C} / \mathrm{m}^{2}\right) \\ d & \text { Emitter-Extractor Separation }(\mathrm{m}) \\ d_{p} & \text { Magnetic Particle Diameter (m) } \\ d_{j} & \text { Jet Diameter (m) } \\ \vec{E} & \text { Electric Field (V/m) } \\ E_{a} & \text { Apex Electric Field (V/m) } \\ \vec{F}_{e} & \text { Coulomb Force (N) } \\ \vec{f}_{m} & \text { Kelvin Force Density }\left(\mathrm{N} / \mathrm{m}^{3}\right) \\ \vec{F}_{m} & \text { Kelvin Force (N) } \\ G(\varepsilon) & \text { Jet scaling function } \\ g_{0} & \left.\text { Standard Gravity (m/s }{ }^{2}\right) \\ H & \text { Magnetizing Field }(\mathrm{A} / \mathrm{m}) \\ I & \text { Emission Current }(\mathrm{A}) \\ I_{s p} & \text { Specific Impulse }(\mathrm{s}) \\ K & \text { Electrical Conductivity }(\mathrm{S} / \mathrm{m})\end{array}$

xvii 


\begin{tabular}{|c|c|}
\hline$k$ & Boltzmann Constant $(\mathrm{J} / \mathrm{K})$ \\
\hline$L(\alpha)$ & Langevin Function \\
\hline$\vec{M}$ & Magnetization (A/m) \\
\hline$M_{c}$ & Critical Magnetization for Normal-Field Instability \\
\hline$M_{d}$ & Saturation Magnetization (A/m) \\
\hline$m$ & Particle/Ion Mass (kg) \\
\hline$\dot{m}$ & Mass flow rate $(\mathrm{kg} / \mathrm{s})$ \\
\hline$m_{f}$ & Final Payload Mass (kg) \\
\hline$m_{0}$ & Initial Payload Mass (kg) \\
\hline$\vec{P}$ & Polarization $\left(\mathrm{C} / \mathrm{m}^{2}\right)$ \\
\hline$P_{i n}$ & Electrical Input Power to Thruster (W) \\
\hline$P_{E}$ & Electric Stress at Apex $(\mathrm{Pa})$ \\
\hline$p$ & Fluid Pressure $(\mathrm{Pa})$ \\
\hline$\Delta p$ & Passive emission pressure gradient $(\mathrm{Pa})$ \\
\hline$Q$ & Volumetric Flow Rate (nL/s) \\
\hline$Q_{r}$ & Rayleigh Charge Limit (C) \\
\hline$q$ & Particle/Ion Charge (C) \\
\hline$R_{0}$ & Characteristic Radius for Bond Number (mm) \\
\hline$R_{c}$ & Critical Apex Radius at Onset (m) \\
\hline$R_{\text {mean }}$ & Mean Curvature (m) \\
\hline$r^{*}$ & Characteristic Jet Radius (m) \\
\hline$r_{L}$ & Larmor Radius (m) \\
\hline$T$ & Temperature (K) \\
\hline$T / P)_{\text {ideal }}$ & Thrust-to-Power Ratio (N/watt) \\
\hline$T_{\text {ideal }}$ & Idealized Thrust (N) \\
\hline $\mathbf{T}_{1,2}$ & Fluid Stress Tensor $\left(\mathrm{N} / \mathrm{m}^{2}\right)$ \\
\hline
\end{tabular}




\begin{tabular}{|c|c|}
\hline $\mathbf{T}_{e}$ & Electric Stress Tensor $\left(\mathrm{N} / \mathrm{m}^{2}\right)$ \\
\hline $\mathbf{T}_{m}$ & Magnetic Stress Tensor $\left(\mathrm{N} / \mathrm{m}^{2}\right)$ \\
\hline $\mathbf{T}_{s}$ & Stokes Stress Tensor $\left(\mathrm{N} / \mathrm{m}^{2}\right)$ \\
\hline$\vec{t}_{e, m}$ & Electric/Magnetic Surface Stress Vector $(\mathrm{Pa})$ \\
\hline$\vec{u}$ & Fluid Velocity Vector (m/s) \\
\hline$\vec{u}_{m e s h}$ & Mesh Interface Velocity $(\mathrm{m} / \mathrm{s})$ \\
\hline$U(z)$ & Energy of Perturbation Surface \\
\hline$U_{g}$ & Gravitational Energy (J) \\
\hline$U_{m}$ & Magnetic Field Energy $(\mathrm{J})$ \\
\hline$U_{s}$ & Surface Energy (J) \\
\hline$V$ & Electric Potential (V) \\
\hline$\forall$ & Droplet Volume $\left(\mathrm{m}^{3}\right)$ \\
\hline$V_{\text {start }}$ & Critical Voltage for Onset of Emission (V) \\
\hline$V_{m}$ & Magnetic Scalar Potential (A) \\
\hline$v_{e}$ & Propellant Exhaust Velocity $(\mathrm{m} / \mathrm{s})$ \\
\hline$\Delta v$ & Change in spacecraft velocity $(\mathrm{m} / \mathrm{s})$ \\
\hline$Z$ & Height for Apex Scaling (mm) \\
\hline$Z_{i m p}$ & Hydraulic Impedance \\
\hline$\alpha$ & Energy Ratio $(m H / k T)$ \\
\hline$\beta$ & Langevin Fit Parameter $(\mathrm{A} / \mathrm{m})$ \\
\hline$\gamma$ & Surface Tension $\left(\mathrm{N} / \mathrm{m}^{2}\right)$ \\
\hline$\varepsilon$ & Material Permittivity $(\mathrm{F} / \mathrm{m})$ \\
\hline$\varepsilon_{0}$ & Permittivity of Free Space $(\mathrm{F} / \mathrm{m})$ \\
\hline$\varepsilon_{r}$ & Relative Permittivity \\
\hline$\eta$ & Coulomb/Kelvin Force Ratio \\
\hline$\eta_{E}$ & Energy Efficiency \\
\hline
\end{tabular}




$\begin{array}{ll}\eta_{i} & \text { Ionization Efficiency } \\ \eta_{p} & \text { Polydisperse Efficiency } \\ \eta_{T} & \text { Total Power Efficiency } \\ \eta_{t r} & \text { Transmission Efficiency } \\ \eta_{\theta} & \text { Angular Efficiency } \\ \mu & \text { Viscosity (mPas }) \\ \mu_{0} & \text { Vacuum Permeability }(\mathrm{V} \cdot \mathrm{s} /(\mathrm{A} \cdot \mathrm{m})) \\ \mu_{r} & \text { Relative Permeability } \\ \rho & \left.\text { Fluid Density (kg/m }{ }^{3}\right) \\ \rho_{I}(\theta) & \text { Angular Current Density }(\mathrm{A}) \\ \sigma_{1}, \sigma_{2} & \text { Fluid Conductivity }(\mathrm{S} / \mathrm{m}) \\ \sigma_{n, \text { elec }} & \text { Surface Normal Electric Stress }\left(\mathrm{N} / \mathrm{m}^{2}\right) \\ \sigma_{n, m a g} & \text { Surface Normal Magnetic Stress }\left(\mathrm{N} / \mathrm{m}^{2}\right) \\ \tau & \text { Langavin Fit Parameter }(\mathrm{m} / \mathrm{A}) \\ \tau_{r} & \text { Electric Relaxation Time }(\mathrm{s}) \\ \chi_{t} & \text { Volume Fraction of Nanoparticles } \\ \nabla_{H} & \text { Gragnetic Susceptibility } \\ & \end{array}$




\section{Abstract}

This dissertation presents three studies on the electrospray of ionic liquid ferrofluid. Ionic liquid ferrofluids are electrically conductive super-paramagnetic fluids which respond strongly in the presence of electric and magnetic fields. When a small reservoir of ionic liquid ferrofluid is positioned within a magnetic field, magnetic stresses will deform the fluid interface into a peak. The addition of a strong electric field will further stress the fluid interface until a threshold stress is reached at which point the surface tension cannot contain the combined stresses and a spray of fluid or ions results at the apex. This process is termed electrospray, albeit a less understood form of electrospray owing to the addition of magnetic stresses which are not present in traditional electrospray.

The first study included in this dissertation presents a computational fluid dynamics model of the combined electro-magnetic instability critical for electrospray. The developed model utilized the static formulation of the Maxwell equations to calculate the Maxwell stress tensor for an ionic liquid ferrofluid. When combined with the Stokes stress tensor, the duo of equations capture the fluid stresses present within the instability. The model was first employed to study the influence of a magnetic field on the onset potential of a capillary needle electrospray source. The simulation predicted onset potential agreed well with the experimentally captured onset under matching field conditions. The numerical tool was then utilized to study the dynamics of sessile ionic liquid ferrofluid droplets. The computational results were verified against laboratory images of sessile drops obtained under matching field conditions. The simulation performed exceptionally up until about $85 \%$ of the onset potential at which point the simulation began to over predict the apex height of the combined instability. 
The second portion of this dissertation consisted of long duration emission studies of an ionic liquid ferrofluid normal-field source. An operational procedure was developed which permitted a source consisting of a single emitter to operate with constant extraction potential for spans in extent of 15 hours. Time-lapse imagery of source enabled the mass flow rate to be approximated, permitting derived propulsion performance parameters to be obtained. Three different magnetic field strengths were investigated, and it was found that the magnetic field strength has no identifiable impact on propulsion performance. On average, the mass flow rate of the source was $28 \mathrm{ng} / \mathrm{s}(15.5 \mathrm{pL} / \mathrm{s})$, with a specific impulse of $1385 \mathrm{~s}$ and a thrust of $0.380 \mu \mathrm{N}$ per emitter. During the telemetry, the sensitivity of the source was analyzed and it was found that for moderate changes in extraction potential the source remained stable, but for increases on the order of $25-30 \%$ of the baseline voltage secondary emission sites were observed to form.

The final set of studies included in this dissertation focuses investigated the angular divergence of ferrofluid electrospray emitting via the normal-field instability. The angular current density was measured through the use of a segmented Faraday probe and quantified in terms of an angular power utilization efficiency factor. For the source, the average power efficiency was found to be $94 \%$. A strong correlation was found between increased emission current and increased mass flow rate and decreased power efficiency. Finally, a very small difference in efficiency was resolved between the positive and negative emission polarities.

The last chapter of this dissertation models the magnitude of the Kelvin and Lorentz forces in the emission plume to determine their potential to influence particle trajectories. It was found that in the apex region, the Coulomb force dominates the Kelvin force by several orders of magnitude - indicating that the Kelvin force is unlikely to affect the trajectories of emitted magnetic particles. It was also found that the magnitude of the Lorentz force in the apex region was too small to influence particle trajectories for even the lightest ions expected. 


\section{Chapter 1 Electric Spacecraft Propulsion}

Electrically powered spacecraft thrusters are a highly efficient means of providing in space propulsion for a spacecraft. This technology utilizes electrical energy to accelerate a propellant which in exchange changes the velocity of a spacecraft. This is typically done by ionizing the propellant and accelerating it using an electric field, or through using an electric current to rapidly heat propellant.* As a result, a very large amount of energy can be used to accelerate a given mass of propellant. Electric propulsion (EP) systems generally acquire their energy from the sun via solar panels; however, nuclear batteries have the potential of providing the required energy.

In contrast, chemical propulsion systems acquire their energy via combustion or catalytic decomposition of propellants. This process is limited by the amount of energy per unit mass of reactants released during the reaction-yielding a barrier on the maximum achievable exhaust velocity. Electric propulsion technologies, free of this heat of reaction barrier, can deliver more energy per unit exhaust mass, thus permitting a higher propellant velocity and mass efficiency.

In 1903, the Russian scientist Konstantin Tsiolkovsky presented an equation relating the propellant velocity, desired change in spacecraft velocity, and mass change of the spacecraft. This relation came to bear his name, although it is worth noting that several

\footnotetext{
* A detailed summary of electric propulsion is provided by Jahn and Choueiri in "Electric Propulsion" in the Encyclopedia of Physical Science and Technology. ${ }^{3}$
} 
previous authors had published the relationship before him. ${ }^{\dagger}$ The Tsiolkovsky equation is also known simply as the ideal rocket equation. This relation reads as follows:

$$
\Delta v=v_{e} \ln \left(\frac{m_{0}}{m_{f}}\right)
$$

Where $\Delta v$ (delta-v) is the change in spacecraft velocity, $v_{e}$ is the propellant velocity, $m_{0}$ and $m_{f}$ are the mass of the spacecraft before and after the maneuver, respectively. The difference between $m_{0}$ and $m_{f}$ is the amount of propellant that is consumed. Simply stated, $\Delta m$ is the required propellant mass necessary to yield a spacecraft velocity change of $\Delta v$ for a given propellant velocity. The relation can also be expressed in terms of the specific impulse, $I_{s p}$, where:

$$
v_{e}=I_{s p} g_{0}
$$

The parameter $g_{0}$ is the standard acceleration of gravity defined at sea level. The specific impulse has units of seconds and is often used as a metric to compare propulsion systems. A higher specific impulse denotes a more efficient use of the propellant mass as demonstrated in Figure 1.1. ${ }^{\ddagger}$ The higher efficiency of the propulsion system can enable (1) a lower launch mass, (2) a higher payload mass, or (3) a longer mission.

\footnotetext{
$\dagger$ In 1813, the British mathematician William Moore published his "Treatise on the Motion of Rockets: To which is Added an Essay on Naval Gunnery in Theory and Practice." Moore's essay presented an equation relating of rocket motion closely resembling Tsiolkovsky's equation. ${ }^{4}$

$\$$ Bipropellant propulsion systems (solid or liquid) have specific impulses on the order of 200468 seconds. Hall effect EP systems range from 1000-1700 seconds. ${ }^{5}$ Advanced Hall effect thruster designs have demonstrated impulses around 3000 seconds. ${ }^{6,7}$ Electrostatic ion engines range from $1200-5000$ seconds. ${ }^{5}$
} 


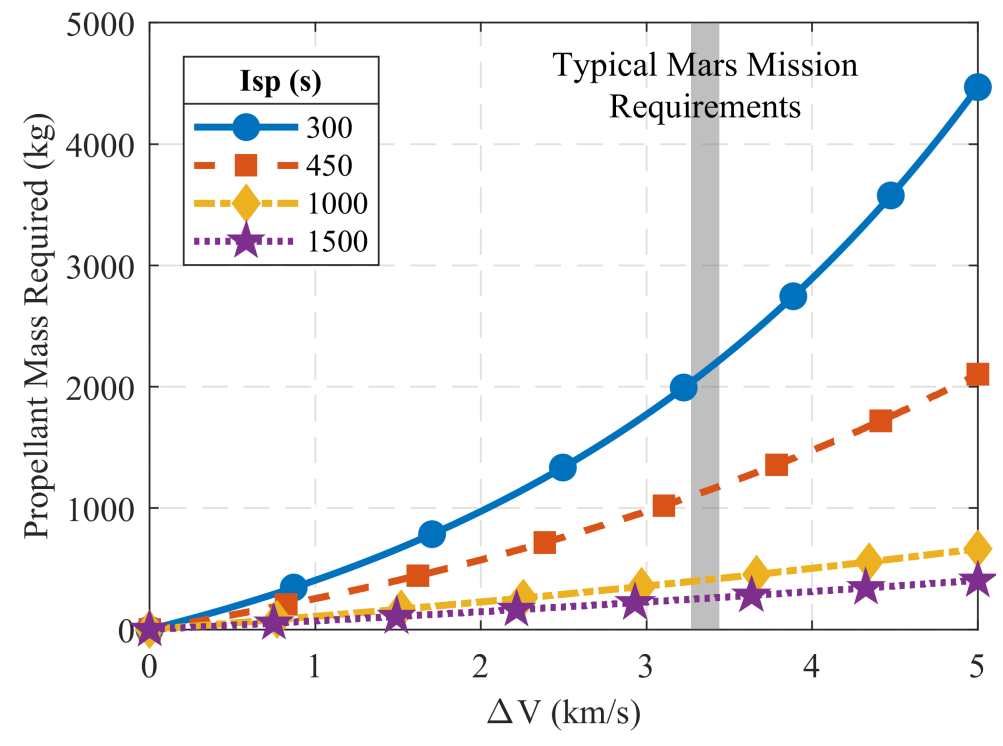

Figure 1.1: Propellant mass requirements to deliver a specific delta-v for a $1000 \mathrm{~kg}$ spacecraft. A spacecraft requiring a delta-v of $3 \mathrm{~km} / \mathrm{s}$ using a propulsion system with an $I_{s p}$ of 300 would require $1772 \mathrm{~kg}$ of propellant. By comparison, a similar mission with a 1500 s $I_{s p}$ propulsion system would require $226 \mathrm{~kg}$.

Generally speaking, electric propulsion systems offer much lower thrust levels than those achievable by chemical means. Any maneuver performed with electric propulsion tends to take a longer duration to achieve the same delta-v which may be undesirable in certain scenarios. The source of propulsion most optimal depends on the mission objectives; however, in the case of interplanetary travel where delta-v requirements are substantial and communication satellites for station keeping, EP obtains a large market share.

Common types of electric propulsion include: gridded ion thrusters, Hall-effect thrusters, resistojets, and arcjets. Spacecraft operating on these technologies have launch masses ranging from 1000-7000 kg. ${ }^{8}$ With the advent of microsatellite (10 to $100 \mathrm{~kg}$ ) and nanosatellites (1-10 kg) a desire for suitable micropropulsion systems has materialized. Micropropulsion systems for this class of satellites are an enabling technology capable of improving mission capability by providing propulsion for orbital maintenance, orbital changes, station keeping, and potentially missions beyond Earth orbit.

One promising solution for electric micropropulsion are colloid/electrospray thrusters. The term colloid thruster is typically reserved to describe electrospray thrusters operating 
in the droplet emission regime. Electrospray thrusters use an electric field to extract droplets or ions from a liquid, which are then accelerated away from the spacecraftimparting a small thrust on the spacecraft. Individual emitters can be manufactured on a sub-millimeter scale, yielding compact scalable thrusters. These thrusters use inert, nontoxic propellant and operate at high specific impulses.

This chapter will investigate the history of electrospray propulsion, the target market for electrospray propulsion, propulsion performance parameters, and ionic liquid ferrofluid electrospray.

\subsection{Application of Electrospray Propulsion}

In 1999, a trio of researchers from California Polytechnic State University and Stanford started what would eventually develop into the cubesat standard nanosatellites. The standard specifies a 1-U 10x10x10 cm cube form factor which integrates satellite with a deployment system, called a P-POD. A satellite can be composed of multiple units, with a $3 \mathrm{U} 10 \times 10 \times 30 \mathrm{~cm}$ form factor being very popular.

The adoption of the standard combined with the increased availability of low-cost commercial off-the-shelf (COTS) electronics and willingness of launch providers to allow ride-along secondary payloads has resulted a rapid growth in CubeSat launches. A nanosatellite database compiled by nanosats.eu shows that as of the $1^{\text {st }}$ of January 2018, 811 satellites abiding by the CubeSat standard have been launched. ${ }^{\S}$ Companies and universities are the leading developers of CubeSats.

Quick to design and build, low-cost and capable of being deployed in large numbers these satellites have potential applications ranging from Earth observation and sensing, communications, and space exploration. Unfortunately, the lack of an efficient propulsion systems has limited CubeSats from reaching their full potential. Differential drag can be used to help phase a constellation of satellites in an orbital plane, ${ }^{9}$ however, drag compensation, plane changes, and raising an orbit all require controllable propulsion.

$\S$ Nanosatellite Database by Erik: http://www.nanosats.eu/index.html\#database 
The CubeSat standard limits the amount of stored chemical energy permitted onboard the satellite without a waiver to protect the primary and other secondary payloads. Because of these strict launch restrictions, few viable propulsion systems exist. Cold-gas thrusters are ineffective at providing any considerable $\Delta v$ to the spacecraft due to their lack of energy. ${ }^{10}$ Traditional electric propulsion systems like Hall-thrusters and gridded ion thrusters are unable to scale to the size levels of these satellites while maintaining a reasonable level of efficiency. Consequently, electrospray propulsion has received considerable attention to provide nanosatellites with in space propulsion.

\subsection{Electrospray Propulsion - Past and Present}

Electrospray was first investigated as a potential means of providing spacecraft propulsion in the 1960s and early 1970s. The early thrusters developed used a glycerol propellant doped with agents to increase their conductivity. ${ }^{11}{ }^{12}$ Early research into electrospray propulsion faded in the 1970s, which has been attributed, in part, to (1) improved performance of competing propulsion systems, (2) an inability to achieve the required levels of thrust, and (3) technical challenges related to the high voltages required to achieve emission using propellants available at the time. ${ }^{13}$ Interest in the technology was recently renewed primarily as a result of miniaturization of spacecraft. Additionally, developments in electrospray ${ }^{13}$ and MEMS manufacturing pioneered by other fields has overcome many of the challenges faced by early researchers.

Various approaches have been employed for the design electrospray thruster. In 2015, MIT developed the Scalable ion Electrospray Propulsion System (S-iEPS) thruster which is manufactured via laser ablation of porous glass to provide hydraulic impedance. ${ }^{14}$ Two graduate students from MIT worked to commercialize this technology which is sold by Accion Systems. The University of California Irvine recently demonstrated a 64 emitter MEMS fabricated microchannel thruster etched out of silicon with microchannels for flow impedence. ${ }^{2}$ Researchers at EPFL also fabricated a thruster using MEMS fabrication but filled the emitter capillary with microbeads to provide hydraulic impedance. ${ }^{15}$ This was by 
no means a comprehensive list of all the research being performed on electrospray thrusters and is simply intended to present a list of the paths being pursued.

Current research utilizes room temperature ionic liquids for the spray fluid, which are often simply referred to as ionic liquids (IL). Ionic liquids are best described as room temperature molten salts. As a result of their ionic forces within the liquid and the low melting point, these fluids have extremely low vapor pressures ${ }^{16}$-a fluid property ideal for electrospray propulsion, otherwise the propellant will evaporate in a vacuum without a heavy pressure vessel or a propellant cooling system. Ionic liquids also have favorably high conductivities ranging from $0.1-1 \mathrm{~S} / \mathrm{m},{ }^{17}$ a range of conductivities enabling the ionic emission electrospray regime to be achieved. ${ }^{18}$ Electrospray propulsion systems operating in the ionic emission mode, rather than the droplet mode, achieve a higher specific impulse while having a lower thrust-to-power ratio. Performance characterization of electrospray thrusters will be elaborated on in the Section 1.3.

From a propellant storage standpoint, ionic liquids have excellent propellant storage densities without using pressurized storage vessels. EMIM-Ntf2 and EMI-BF 4, two common ionic liquids used in electrospray propulsion research, have densities of 1523 $\mathrm{kg} / \mathrm{m}^{3}$ and $1294 \mathrm{~kg} / \mathrm{m}^{3}$, respectively. ${ }^{19}$ By comparison, Xenon, a common propellant utilized in Hall-effect and gridded ion thrusters at $50{ }^{\circ} \mathrm{C}$ requires a storage pressure of 126 bar to obtain storage density of $1500 \mathrm{~kg} / \mathrm{m}^{3}$.

In December 2015, Busek Co. Inc became the first company to have a flight-qualified electrospray thruster launch on the LISA Pathfinder spacecraft. ${ }^{20}$ In November 2016, an electrospray thruster developed by the Space Propulsion Lab at MIT was launched aboard two AeroCube 8 cubesats, built by the Aerospace Corporation. At the time of writing, no published work regarding the on-orbit performance of these thruster was found in the literature, likely a consequence of the recent mission launch dates.

\subsection{Performance Characterization}

To analyze the propulsive performance of an electrospray thruster, we will first investigate an idealized thruster. For this, we will consider an electrospray thruster which 
emits a single species of particles with a mass $m$ and charge $q$ and have an initial electrical potential $V$. This can be considered an ideal electrospray thruster and provides an excellent metric for the role of particle mass-to-charge ratios on power and thrust. As the particles travel through the electric field towards the extractor electrode, electrical potential energy is converted into kinetic energy - accelerating the particles.

$$
\frac{1}{2} m v_{e}^{2}=q V \quad \Rightarrow \quad v_{e}=\sqrt{2 \frac{q}{m} V}
$$

If the emitted particles possess no off-axis velocities, thrust produced by the electrospray source becomes:

$$
T_{\text {ideal }}=\frac{d}{d t}\left(m \vec{v}_{e}\right)=I \sqrt{\frac{2 V}{q / m}}
$$

where $I$ is the total emission current. Substituting Eq. (1.3) into Eq. (1.2) yields the ideal specific impulse for this electrospray thruster:

$$
I_{s p}=\frac{v_{e}}{g_{0}} \Rightarrow I_{s p, \text { ideal }}=\frac{1}{g_{0}} \sqrt{2 \frac{q}{m} V}
$$

If no electrical inefficiencies are present within the system, the thrust-to-power ratio becomes:

$$
\left.\frac{T}{P}\right)_{\text {ideal }}=\sqrt{\frac{2 m}{q V}}
$$

From Eq. (1.4), it can be observed that thrust can be increased by increasing emission current, the mass-to-charge ratio of the emitted particles, or by increasing the extraction voltage. However, increasing the mass-to-charge ratio has the adverse effect of decreasing specific impulse (Eq. (1.5)) while increasing the thrust to power ratio (Eq. (1.6)). The optimal propellant and electric propulsion technology will ultimately depend on more than 
just specific impulse. Factors including mission requirements, power limitations, ionizing energy, and storage capacity must all be considered. For example, a higher thrust, lower specific impulse thruster operation could be desirable if a more rapid orbital transfer is acceptable at the cost of more propellant.

The efficiency at which the available electrical power on the spacecraft is utilized in the production of thrust can be described by the thrust power efficiency factor $\eta_{T}$ :

$$
\eta_{T}=\frac{T^{2}}{2 \dot{m} P_{i n}}
$$

For the idealized thruster model discussed previously in the section, $\eta_{T}$ will simply be 1 . However, the assumption that all particles are emitted at the same mass-to-charge ratio deviates significantly from what is observed in practice for electrospray and colloid thrusters. Additionally, emitted particles are not born at a uniform potential $V$ and often have off-axis velocity components.

Lozano presented a model to describe the efficiency of a thruster that bridges the gap between the idealized thruster previously discussed and a physical thruster. In his model, the thrust efficiency is expressed as a product of individual factors which contribute to the overall inefficiency. ${ }^{21}$ This relation becomes:

$$
\eta_{T}=\eta_{i} \eta_{t r}^{2} \eta_{\theta} \eta_{E} \eta_{P}
$$

in which $\eta_{i}$ and $\eta_{t r}$ are the ionization and transmission efficiencies, respectively. These terms account for account for inefficiencies in ionizing all of the available propellant and intercepted current by the extractor or accelerator. The angular power efficiency is expressed as $\eta_{\theta}$, while $\eta_{E}$ is the energy efficiency, which accounts for the inefficiencies resulting from not accelerating a particle to the full extraction potential. Finally, $\eta_{P}$ is the polydispersive efficiency, which accounts for energy wasted accelerating particles of different charge-to-mass ratios. Note: Eq. (1.8) makes the assumption that the efficiency 
factors are completely decoupled and that there is, for example, no angular dependence in the polydispersive efficiency. In practice, some terms are observed to have angular dependence; however, this model serves as a concise statement of various factors impacting efficiency.

Real thrust production and power efficiency can be determined experimentally from one of two approaches; through direct measurement or indirectly by determining angular divergence and simultaneous measuring the distribution of the charge-to-mass ratio and electric potential. A direct measurement of thrust can be difficult to achieve. Thrust produced by existing electrospray thrusters, which contain multiple thruster units, ranges up to $100 \mu \mathrm{N} .^{22}$ The precise design, calibration, and measurement noise intrinsic of these systems presents formidable challenges. However, thrust stands have been developed to achieve measurements in this range. ${ }^{23,24}$

Indirect measurements to obtain a thrust are not free of imposing challenges. The comprehensive set of measurements necessary with minimal approximation would require the net emission current and mass-to-charge ratio spectrum at each retarding potential. These measurements must be taken at each angular location within the spray. Such a compilation of measurements could be obtained by integrating a retarding potential analyzer with a time-of-flight mass spectrometer. However, certain assumptions can be employed to simplify indirect measurements with only minimal loss in accuracy., 25

\subsection{Ionic Liquid Ferrofluid Electrospray Thrusters}

In 2013, Meyer and King at Michigan Technological University were the first to demonstrate that electrospray emission can be achieved ionic liquid ferrofluids (ILFF). ${ }^{26-}$ ${ }^{28}$ At the time of their research, only one other publication could be found to exist in the literature dealing with electrospray from any ferrofluids. This research, conducted by Mkrtchyan et al, used a very low conductivity fluid. ${ }^{29} \mathrm{~A}$ ferrofluid is an intriguing class of colloidal fluid. These fluids exhibit super paramagnetic behavior, and will be discussed in detail in Chapter 3. For now, these fluids are special because they exhibit an ability to form sharp peaks in the presence of a magnetic field. Meyer and King's technique utilizes this 
ferrofluid instability to create the initial concentration in the electric field necessary to achieve electrospray emission. When an electric field is applied to this formation, the peaks grow and become progressively sharper until electrospray emission can be achieved from one or more of the peaks. The process of emission is discussed in Figure 1.2:

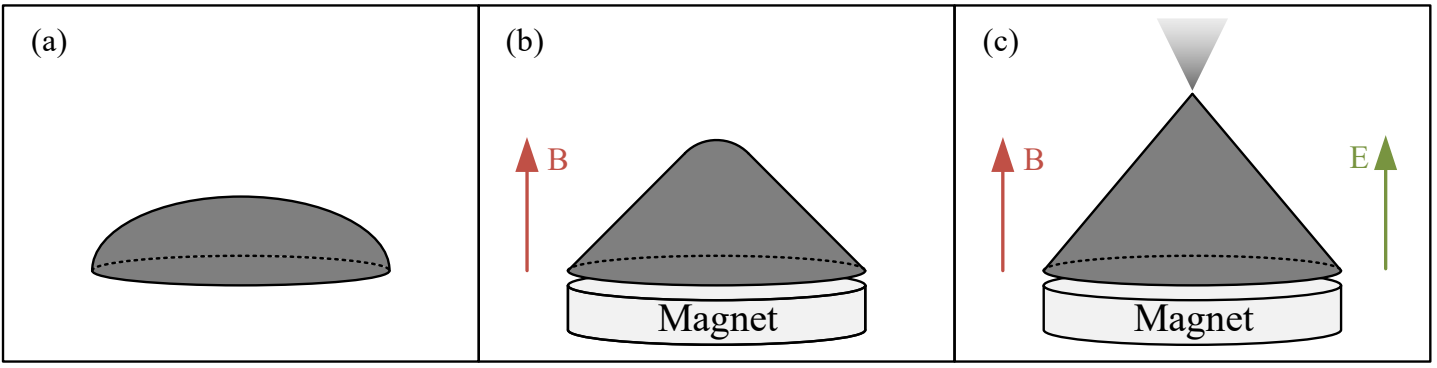

Figure 1.2: Electrospray emission from a combined ferro-electrohyrodynamic instability: (a) In the absence of an applied field, a sessile drop of ILFF spreads. (b) The application of a magnetic field stresses the fluid interface resulting in the fluid rising into a peak. (c) Finally, the addition of a strong electric field further stresses the fluid interface until emission results. The onset potential required for the combined ferro-electrohydrodynamic instability is less than the potential required to emit from configuration (a) as a result of the preconditioning provided by the magnetic stresses isolated in (b).

Meyer developed a 5-tip electrospray emitter. This emitter struggled to achieve steady simultaneous emission from all emission sites, although this is not an uncommon issue in electrospray. During this research, Meyer demonstrated that his ferrofluid electrospray emitter was capable of self-repair after a destructive event such as emitter to extractor arcing. In such a case, the fluid was observed to simply reflow towards the emission site, the peak would reform, and emission would restart. Work by Meyer also investigated the spacing between the normal-field instability in non-uniform fields. ${ }^{30}$ 


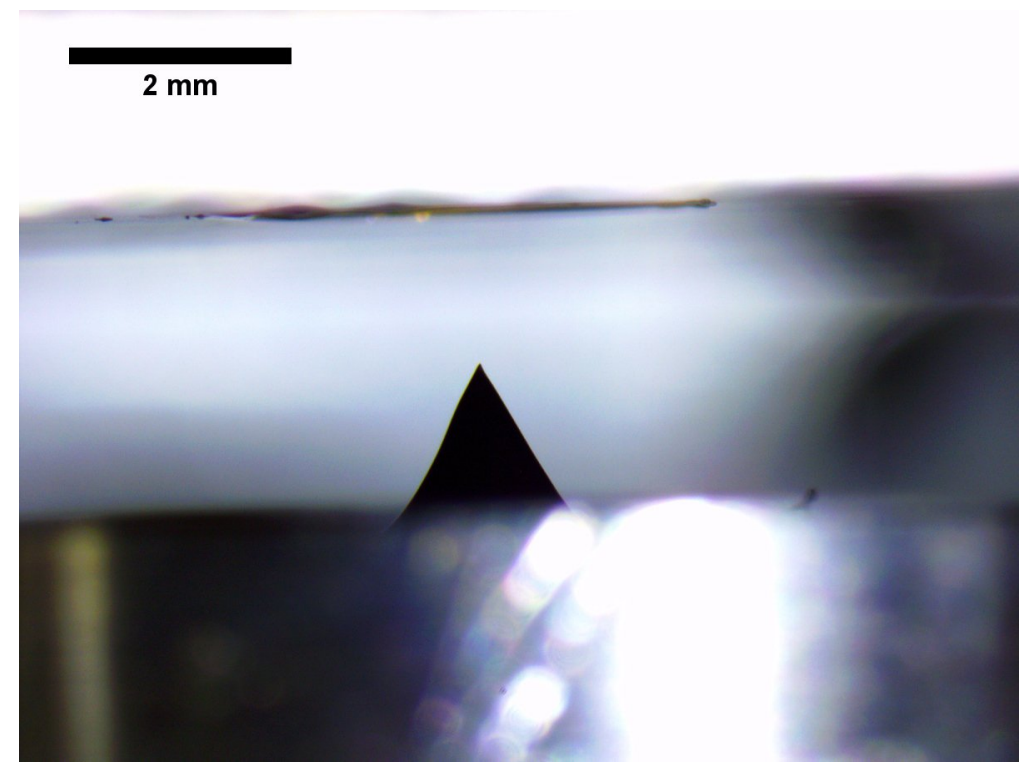

Figure 1.3: Electrospray emission from an emitting peak formed using a combined magneto-electric instability.

Kurt J. Terhune conducted additional work in ionic liquid ferrofluid electrosprays. Work performed by Terhune focused primarily on emission from capillary needle sources such that comparisons could be made against traditional electrospray emitters. Terhune's work collected time-of-flight mass spectrometry measurements and retarding potential analyzer (RPA) spectrum for ferrofluid of varying nanoparticle concentrations in magnetic fields. ${ }^{31-35}$ Terhune found that the minimum stable flow rate of a capillary source decreased when an ionic liquid ferrofluid was sprayed in the presence of a magnetic field. Experiments performed by Madden discovered a similar trend. ${ }^{36}$ Terhune also performed time-of-flight mass spectrometry measurements on a normal-field emission source, similar to that shown in Figure 1.3, and found the source to emit in a mixed ion-droplet mode. 



\section{Chapter 2 Overview of Electrospray and Electrohydrodynamics}

When a strong electric field is applied to a conductive, or dielectric liquid drop, which is surrounded by an insulating medium, a droplet will stretch due to the electric field. The interface geometry of the droplet will be a balance between the capillary stress, internal pressure, and the electric stresses. For the case of a highly conductive fluid at equilibrium, the interface balance becomes:

$$
\Delta p+\frac{1}{2} \varepsilon_{0}(\vec{E} \cdot \hat{n})^{2}=2 \gamma R_{\text {mean }}
$$

where $\Delta p$ is the difference in hydrostatic pressure across the fluid interface, $(1 / 2) \varepsilon_{0} E_{n}^{2}$ is the electric stress on the interface, and $2 \gamma R_{\text {mean }}$ is the capillary stress. The component $R_{\text {mean }}$ is the mean curvature at the interface location.

For a sufficiently strong electric field, a condition can be achieved whereby the electric stresses will become too large to be constrained by the capillary stress. At this point, the meniscus will form into a structure known as a Taylor cone. ${ }^{37}$ When this limit is reached, a phenomenon occurs whereby a spray of fluid results at the apex of the deformed meniscus which will then accelerate in the direction of the applied field. This process has been termed electrospray.

To reduce the electric potential required to achieve emission, a supporting structure such as a capillary or externally wetted needle is used to enhance the electric field. The emission from the meniscus can be a jet, ions, droplets, or a combination of the previous. 
The mode of emission depends deeply on the fluid properties, emitter type, and the operation mode of the emitter. ${ }^{18,38-40}$

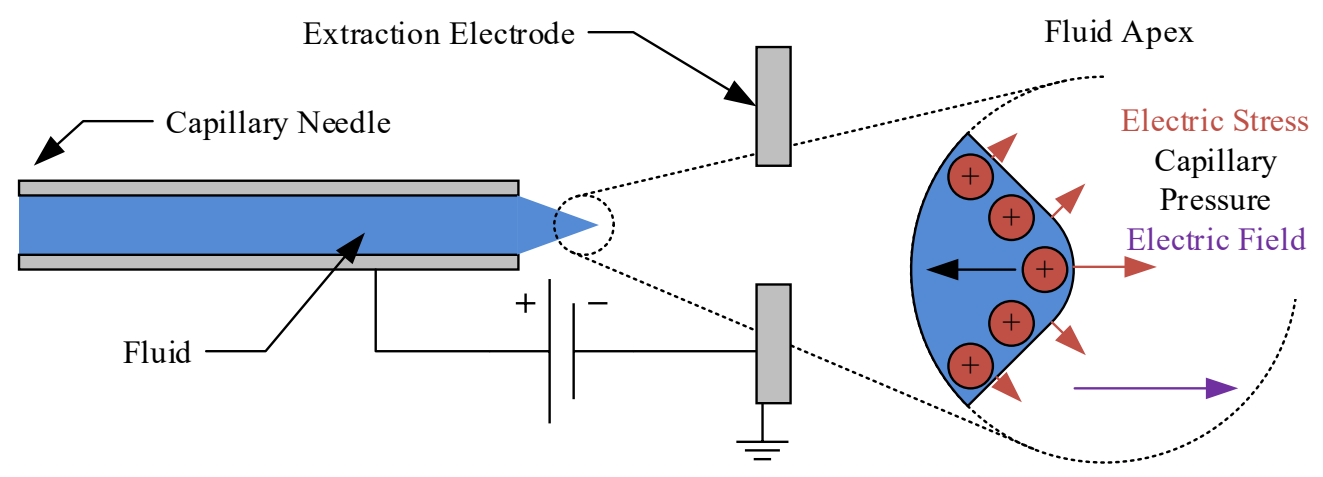

Figure 2.1: (Left) Diagram of an electrospray emission source. The fluid meniscus is stretched by applying an electric potential between the capillary needle and extraction electrode. (Right) Sectional view of the fluid meniscus.

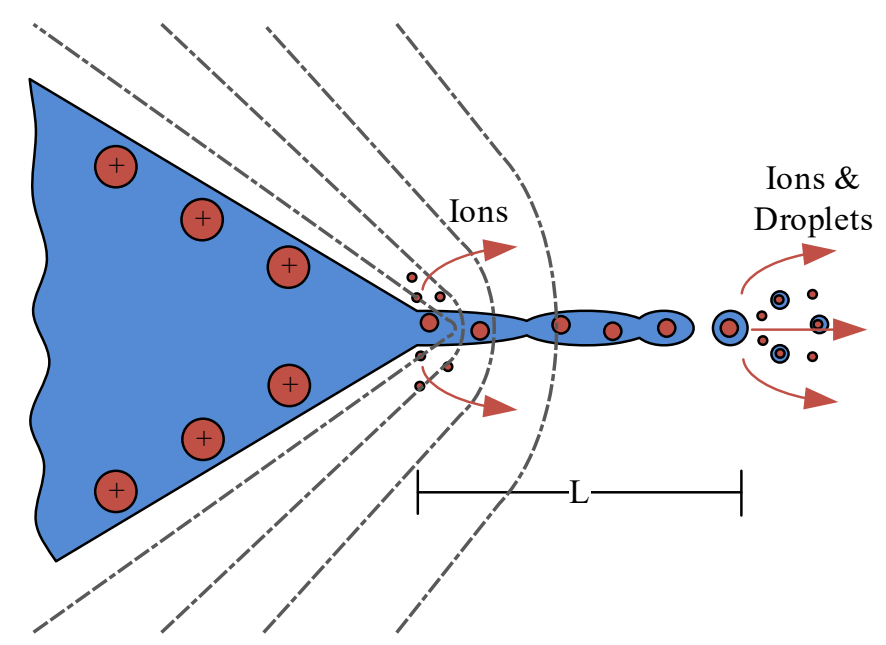

Figure 2.2: Fluid meniscus of an emitting Taylor cone. Emission can take the form of a jet, ionic emission, or a combination of both. The mode of emission that develops will depend on the emitter configuration and fluid properties. The jet length $L$ is a function of the fluid conductivity and surface tension. ${ }^{41}$

Figure 2.1 shows the basic setup of an electrospray apparatus. In this setup, fluid is fed to the emission site through a capillary needle. An electric field applied between the needle and extraction electrode stressed the fluid meniscus until a cone forms. Once the electric stress overcomes the surface tension emission will occur, a process which is illustrated in Figure 2.2. Depending on the properties of the sprayed fluid, a jet may form, the length of which is found to be strongly dependent on the conductivity and surface tension of the 
fluid. Higher conductivities and higher surface tensions yields in a shorter jet length. ${ }^{39,41}$ For high conductivity liquids, like those of liquid metals or ionic liquids, pure ionic emission can be achieved for which droplet and jet formation does not occur. ${ }^{38,39}$ For ion emission to develop, electric fields on the order of $1 \mathrm{~V} / \mathrm{nm}$ are necessary. ${ }^{42}$

The spray illustrated in Figure 2.2 will experience a certain level of divergence. This divergence can result from numerous factors, originating within the plume or at the location of emission. Within the plume, space charge will result in a self-repulsion force which can result in a radial spreading of the beam. Likewise, fragmentation of emitted ion clusters and droplets can produce offspring with strong mutual repulsion capable of providing radial velocity components. The emission dynamics, illustrated in Figure 2.2, also has considerable influence on the spray distribution. At the emission site, the geometry of the cone-jet region can produce areas of intense electric field with considerable radial components, which can generate a non-zero radial term in the velocity vector of the emitted ions and droplets.

\subsection{History of Electrospray}

The first observations of this phenomena were taken centuries ago by Jean-Antoine Nollet at a time when the understanding of electricity was still in its infancy. In 1750, Nollet, comically noted that "a person, electrified by connection to a high-voltage generator, would not bleed normally if he were to cut himself; blood would spray from the wound." *44 One of the early pioneers of electrospray was John Zeleny, who did research focusing on both liquid metal and non-metallic liquid sprays. ${ }^{45,46}$

Possibly the most renowned researcher of electrosprays is G.I. Taylor who developed the theoretical foundation of electrospray physics. Taylor is perhaps best known for his

\footnotetext{
* Jean-Antoine Nollet conducted numerous experiments with electricity. In one experiment, he attempted to measure the speed of electricity by connecting 700 people in a human chain and measuring how quickly the shock propagation through the chain. Nollet also used electricity to try and heal paralysis and control growth rate as well as studying the sweating behavior of electrified animals. ${ }^{43}$
} 
mathematical description in his 1964 paper titled "Disintegration of Water Droplets in an Electric Field." This analysis yielded the $49.3^{\circ}$ half angle limit for the Taylor cone interface. ${ }^{37,47,48}$ The theory developed can also be used to describe electrospray emission from needle sources.

In 2002, the Nobel Prize in chemistry was awarded in part to John Fenn, whose research in electrosprays is utilized in mass spectrometry systems to identify large biological molecules. ${ }^{49}$ In addition to being a propulsion source for satellites, other applications of electrosprays include: pharmaceutical production, nano-manufacturing, liquid-metal ion sources.

\subsection{Electric Stress Tensor}

Before proceeding to discuss the electrostatic stress tensor, it may be helpful to briefly review concepts in electrostatics. For an electrically insulating material, the effect of the electric field causes bound electrons within a molecule to separate, resulting in each molecule possessing a dipole moment. The polarization field, $\vec{P}$, is a measure of this dipole moment per unit volume. The combination of the electric field, $\vec{E}$, and polarizing field yield the displacement field $\vec{D}$. The displacement field can also be expressed in terms of the relative permittivity $\varepsilon_{r}$ per Eq. (2.2).

$$
\vec{D}=\varepsilon_{0} \vec{E}+\vec{P}=\varepsilon_{r} \varepsilon_{0} \vec{E}
$$

For non-linear mediums, the relative permittivity can depend on the electric field. The resulting stress tensor, $\mathbf{T}_{e}$, for such a non-linear incompressible fluid becomes: ${ }^{50}$

$$
\mathbf{T}_{e}=\left\{-\vec{E} \cdot \vec{D}+\int_{0}^{\vec{D}} \vec{E} \cdot d \vec{D}\right\} \mathbf{I}+\vec{D} \vec{E}
$$




\subsubsection{Electric Body Forces}

The electric body force density on an element of fluid is equivalent to the divergence of the electric stress tensor:

$$
\vec{f}_{e}=\nabla \cdot \mathbf{T}_{e}=-q \vec{E}-\frac{1}{2} E^{2} \nabla \varepsilon+\nabla\left(\frac{1}{2} \varepsilon a E^{2}\right)
$$

The first term, $q \vec{E}$ is the coulomb force and is dependent on the free charge density $q$. The second term is the dielectric force and results when a non-homogeneity exists in the dielectric medium. The third term is the electrostrictive pressure where $a$ is a compressibility coefficient of the dielectric fluid, equal to $a=(\rho / \varepsilon)(\partial \varepsilon / \partial \rho)_{T} \cdot{ }^{50}$ For a material that lacks free charge, the first term becomes zero. Likewise, for an incompressible medium, the third term is also zero. Finally, for the work presented in this dissertation, no factors are present (e.g., temperature gradient or colloid concentration gradient) to result in a gradient in the dielectric constant within the fluid. Therefore, it is safe to assume that no electric body forces are present in the work entailed herein.

\subsubsection{Electrohydrodynamics Interface Stress Component}

In this subsection the interfacial stress will be presented. It will be assumed that one of the fluids is a perfect electrical conductor. One parameter to consider is the relaxation time, which is an approximation of the time required for free charge to migrate towards the surface of the conductor. The relaxation time is a function of material conductivity and permittivity and represented as: ${ }^{.1}$

$$
\tau_{r}=\frac{\varepsilon_{r} \varepsilon_{0}}{K}
$$

where $K$ is the fluid conductivity. The conductivity of ionic liquids are frequently on the order of $1 \mathrm{~S} / \mathrm{m},{ }^{17}$ while the dielectric constant of these fluids can be on the order of $10 .{ }^{52}$ 
The resulting electrical relaxation time, $\tau_{r}$, is on the order of $1 \times 10^{-10}$ seconds, a time frame much faster than the fluid relaxation time for a non-emitting meniscus for which the subsequently presented equations are applicable. ${ }^{53}$ Therefore, it is fair to assume the fluid to be a perfect electrical conductor and there is no internal electric field before onset of emission.

Consider the fluid interface shown in Figure 2.3, discontinuities in the electric components of the Maxwell stress tensor over the fluid surface, resulting from a change in materials, results in a stress on the fluid interface.

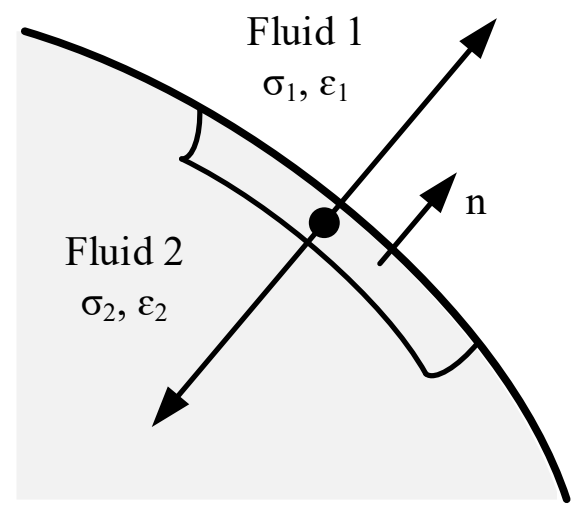

Figure 2.3: Fluid interface of high permittivity fluid (Fluid 2) with low permittivity fluid (Fluid 1).

The normal and tangential components of the electric surface stress can be determined in terms of the stress tensor of both medium evaluated along the interface. The stress vector along the fluid interface becomes:

$$
\vec{t}_{e}=\hat{n} \cdot \mathbf{T}_{e, 1}-\hat{n} \cdot \mathbf{T}_{e, 2}
$$

This stress tensor can be resolved into its respective normal and tangential components. For now, only the normal component will be investigated:

$$
\sigma_{n, \text { elec }}=\hat{n} \cdot \mathbf{T}_{e, 1} \cdot \hat{n}-\hat{n} \cdot \mathbf{T}_{e, 2} \cdot \hat{n}
$$

Substituting Eq. (2.3) into Eq. (2.7), the electric surface stress becomes: 


$$
\sigma_{n, \text { elec }}=\left(\varepsilon_{1} E_{n, 1}^{2}-\varepsilon_{2} E_{n, 2}^{2}\right)-\frac{1}{2}\left(\varepsilon_{1} E_{1}^{2}-\varepsilon_{2} E_{2}^{2}\right)
$$

A step-by-step approach for this derivation can be found in Appendix A.

If medium 2 is highly conductive, the previous equation can be greatly simplified. For a perfect conductor, the internal electric field is zero. Additionally, applying the tangential constraint from the electric field boundary conditions yields: $E_{t, 1}=E_{t, 2}=0$. The simplified form for a highly conductive fluid becomes:

$$
\sigma_{n, \text { elec }}^{\text {conductor }}=\frac{1}{2} \varepsilon_{0} E_{1}^{2}
$$

When a similar approach is utilized to determine the tangential stress components, it can be found that in the absence of surface charge, there is no tangential stress, regardless of the conductivity of the fluid. Consequently, electric tangential stress does not play an active role in the equilibrium interface of a ferrofluid. A full proof for this relation can be found in Appendix A.

The equilibrium geometry of a fluid interface is dependent on the fluid surface tension, interface stresses, and static pressure difference over the interface. This equilibrium is described by the following differential equation:

$$
\hat{n} \cdot \mathbf{T}_{1}-\hat{n} \cdot \mathbf{T}_{2}=\gamma\left(\nabla_{t} \cdot \hat{n}\right) \hat{n}-\nabla_{t} \gamma
$$

In which $\mathbf{T}$ is the fluid stress tensor, $\gamma$ is surface tension between the two fluids, and $\nabla_{t}$ is the tangential gradient along the fluid interface. If one fluid is assumed to be a perfect conductor, substituting the terms in Eq. (2.3) into Eq. (2.10) yields the electrically augmented Young-Laplace equation when one fluid is a perfect conductor:

$$
\begin{aligned}
\Delta p+\frac{1}{2} \varepsilon_{0} E_{1}^{2} & =-\gamma\left(\nabla_{t} \cdot \hat{n}\right)-\nabla_{t} \gamma \\
& =2 \gamma R_{\text {mean }}
\end{aligned}
$$


If there is no gradient in the surface tension, the capillary force can be expressed as $2 \sigma R_{\text {mean }}$ where:

$$
2 R_{\text {mean }}=-\nabla_{t} \cdot \hat{n}
$$

Gradients in surface tension can result from a temperature gradient along the interface or due to a gradient in the concentration of a dissolved species (e.g. Marangoni effect). 


\section{Chapter 3 Ferrofluids and Ferrohydrodynamics}

Ferrofluids, also referred to as magnetic fluids, are superparamagnetic liquids. In the absence of any magnetic field, these fluids behave like any other fluid. When in presence of a magnetic field, they become strongly magnetized. The magnetization of these fluids is a consequence of nanoscale ferromagnetic particles suspended within the fluid which respond to an externally applied field. The resulting magnetic stress tensor adds additional physics to the fluid dynamics when the fluid is present within a magnetic field. The study of ferrohydrodynamics is dedicated to this phenomenon.

Within this chapter, the terms ferromagnetic and paramagnetic will be used frequently. Ferromagnetic materials have magnetic domains, large-scale order, and demonstrate permanent magnetism. However, when ferromagnetic materials are heated, the magnetic properties degrade with increasing temperature and subside completely at the Curie temperature. For all known ferromagnetic materials, the Curie temperature is less than the melting temperature - preventing a genuinely ferromagnetic fluid.

A paramagnetic material does not exhibit magnetic domains or large scale order, but will become magnetized in the presence of a magnetic field. Weakly paramagnetic fluids can be found to naturally occur. Liquid oxygen, as well as certain salt solutions like manganese (II) nitrate, for example, exhibit a weak paramagnetic attraction to a magnet. This attraction requires strong magnetic field gradients. Ferrofluids exhibit a much stronger response, with a magnetic susceptibility, $\chi_{m}$, orders of magnitude higher than natural paramagnetic liquids $\left(\chi_{m}=1.56\right.$ for Ferrotec EFH1 at room temperature compared to $\chi_{m}=3.44 \times 10^{-3}$ for liquid oxygen at 90 Kelvin). ${ }^{54}$ 
Ferrofluids are composed of nanoscale single-domain ferromagnetic particles ${ }^{*}$ coated with a surfactant and suspended within a carrier fluid to form a colloid. The surfactant helps to suspend the particles the carrier liquid, which is most often water or long-chain liquid hydrocarbons. The simplest surfactants are typically composed of long chain molecules. The head of these molecules attach to the nanoparticle surface while the tail has similar chemical and physical properties as the carrier fluid which enables the tail to mix with the carrier fluid. More advanced surfactants can be used which contain polymer chains containing individual structures akin to the single chain surfactant. ${ }^{55}$ Additionally, the surfactant separates the magnetic particles-inhibiting mutual attraction and agglomeration of the particles. Brownian motion prevents the particles from clumping or settling, even in the presence of strong magnetic and gravitational fields.

In the absence of an external magnetic field, the particles are randomly orientated and their rotational motion is dominated by kinetic energy. When an external field is applied, particles will rotate such that their magnetization aligns with the direction of the local field. The collective effect of these ferromagnetic particles results in the liquid exhibiting a superparamagnetic behavior. This process is illustrated in Figure 3.1 and a mathematical relation describing the arrangement of the particles with the local field is presented in Section 3.1 .

The first patent for a ferrofluid was filed by Stephan Papell in $1963 .{ }^{56}$ In his patent, Papell created a kerosene based fluid. He envisioned the magnetic properties of this fluid could be harnessed to draw the ferrofluid (i.e. propellant) towards the turbo pump inlet for a rocket engine in a weightless environment. Papell's vision was never developed. However, since their genesis, these magnetic fluids have found numerous applications including cooling and dampening of audio speakers, ${ }^{57}$ rotary seals,${ }^{58}$ and self-lubricating bearings. ${ }^{59}$ In the bio-medical fields, ferrofluids have received considerable attention for applications such as targeted drug delivery, ${ }^{60}$ MRI contrast agents, and magnetic hyperthermia ${ }^{61}$ - a promising potential cancer treatment which involves localized heating of targeted tissue with the use of magnetic fluids and alternating magnetic fields.

\footnotetext{
* Iron, Nickel, Cobalt, and Magnetite $\left(\mathrm{Fe}_{2} \mathrm{O}_{3}\right)$ all demonstrate ferromagnetism.
} 


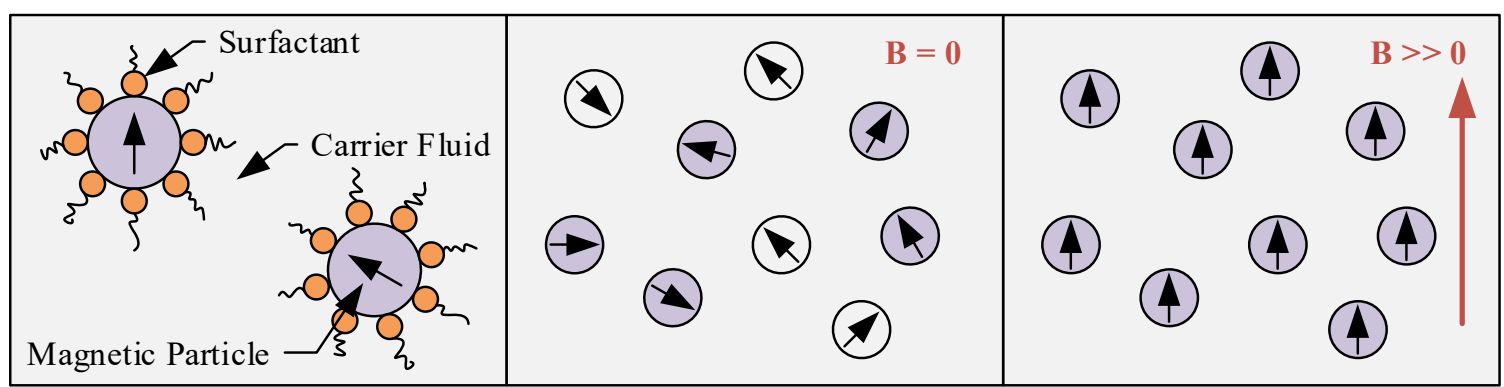

Figure 3.1: (Left) Magnetic nanoparticles are coated with a surfactant, which serves to suspend the particles in the carrier fluid. (Center \& Right) Particles are randomly oriented in the fluid in an absence of a magnetic field. In the presence of the field, particles align with the local field direction.

These fluids demonstrate several documented instabilities, e.g. normal-field instability, thermomagnetic convection, and the fingering instability. Possibly the most eminent of these instabilities is the normal-field instability. This instability results when these fluids are subjected to a magnetic field, a series of valleys and peaks form, which is also often referred to as the Rosensweig instability. The shapes of these peaks are dependent on the fluid properties of the ferrofluid and the nature of the applied magnetic field.

Although ferrofluids have existed since the 1960s, vacuum-based applications have been hindered since typical carrier fluids will evaporate rapidly in a low-pressure environment. In 2011, a new class of ferrofluids was synthesized, using ionic liquids as the carrier fluid. These liquids are referred to as ionic liquid ferrofluids (ILFF). The first stable ionic liquid ferrofluid was developed by Jain, Zhan, and Hawkett. ${ }^{62}$ Since then, stable ferrofluids with ionic liquid carrier fluids have been synthesized by various researchers. ${ }^{63-}$ ${ }^{65}$ When magnetic nanoparticles are suspended within an ionic liquid, the colloid maintains the low vapor pressure, high conductivity, and a viscosity comparable to that of the parent ionic liquid, as well as becoming superparamagnetic. The properties of these fluids make them well-suited for the requirements of electrospray propulsion. The paramagnetic ionic liquid maintains its ability to be stressed by electric fields while gaining the ability to be stressed by a magnetic field. 


\subsection{Magnetic Properties of Ferrofluid}

The behavior of magnetic nanoparticles in a ferrofluid are liken to molecules in a paramagnetic gas. If no field is present, only thermal aggregation influences particle alignment. Consequently, the particles are randomly oriented, and the medium exhibits no magnetic behavior. As a magnetic field is applied, particles are inclined to align in the direction of the field and contribute to the overall strength of the field. The strength of this inclination is dependent on the ratio between the magnetic energy and the thermal energy of the particle. As the magnitude of the field strength increases, the probability of the particles aligning with the applied field increases until a point at which saturation can be achieved. The mathematical relationship describing this process is adapted from Langevin's classical theory of magnetism and can be modeled as follows:

$$
L(\alpha)=\frac{M}{\phi M_{d}}=\operatorname{coth} \alpha-\frac{1}{\alpha}
$$

Where the energy ratio $\alpha$ is:

$$
\alpha=\frac{\pi}{6} \frac{\mu_{0} M_{d} H d_{p}^{3}}{k T}=\frac{m H}{k T}
$$

Given that $L(\alpha)$ denotes the Langevin function, $M$ is the magnetization of the ferrofluid, $\phi$ is the volume fraction of the magnetic nanoparticles, $M_{d}$ is the saturation magnetization of the material of which the nanoparticles are composed. The derivation of this relation can be found in Chapter 2 of Ferrohydrodynamics by R.E. Rosensweig. ${ }^{55}$ The Langevin relation for increasing particle sizes is presented in Figure 3.2: 


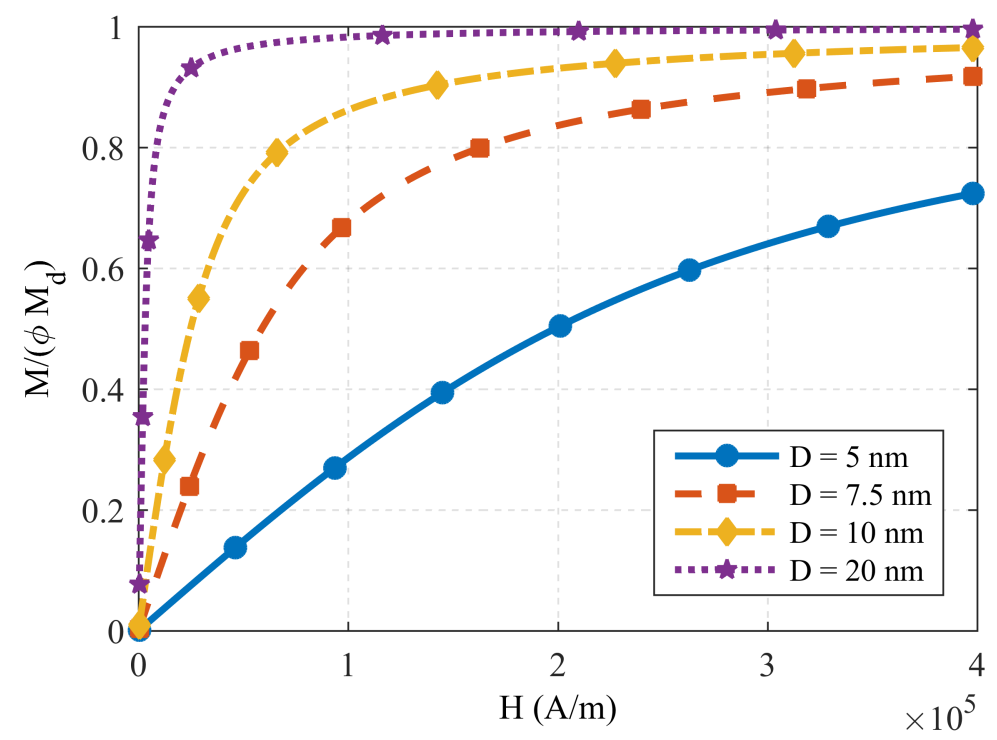

Figure 3.2: Ideal magnetization curves for magnetite nanoparticles $\left(\right.$ For $M_{d}=$ $4.45 \times 105 \mathrm{~A} / \mathrm{m})$

From the previous figure, it can be seen that the magnetization ratio, $M / \phi M_{d}$, increases as particle size increases for the same field strength. This relationship is limited since an upper limit exists on particle size to prevent settling of the particles.

The magnetization curves presented in Figure 3.2 can be obtained for a real ferrofluid using a vibrating sample magnetometer (VSM). The VSM magnetizes a small sample of magnetic material such that it can be approximated as a magnetic dipole. The sample is then vibrated in a direction perpendicular to the applied field. A pair of pickup coils centered on an axis of vibration pick up an induced voltage from the vibrating sample. From the induced voltage in the coils, the dipole moment and fluid magnetization of the sample can be derived ${ }^{66}$ By sweeping through a range of applied field strengths, the magnetization curve can be as obtained at a specified temperature.

For real ferrofluids, the mathematical description presented in Eq. (3.1) may not sufficiently describe fluid magnetization through the range of zero field to fluid saturation. When considering paramagnetic gasses like oxygen, all the molecules are virtually identical, thus the Langevin relation performs extremely well describing observed behavior. Within real ferrofluids, the nanoparticles have a distribution of sizes and a range of magnetic moments ${ }^{67}$ Therefore, it may be necessary to fit only small sections of the 
curve with the Langevin relation or use a non-physical relation, such as a high-order polynomial, to obtain an adequate correlation.

Knowledge of the $M(H)$ relationship for a ferrofluid is an essential component the fluid magnetic stress tensor. The fluid stress tensor is presented in detail in the next section.

\subsection{Magnetic Stress Tensor}

Before proceeding further, it becomes helpful to discuss three quantities in magnetism that can be somewhat ambiguous. These terms are $\vec{B}, \vec{M}$, and $\vec{H}$, and are related by the following relation:

$$
\vec{B} \equiv \mu_{0}(\vec{H}+\vec{M})
$$

As previously introduced, $\vec{M}$ is the material magnetization. However, there is a lack of consensus in the naming of the terms $\vec{B}$ and $\vec{H}$. The $\vec{B}$ term, having units of gauss, is often referred to as the magnetic field, magnetic flux density, or magnetic induction. Meanwhile, the term $\vec{H}$, having units of $A \cdot m^{-1}$, is called the magnetic field, magnetic field intensity, or the magnetizing field ${ }^{68}$ For consistency in this dissertation, $\vec{B}$ will be referred to as the magnetic field, and $\vec{H}$ will be referred to simply as the H-field or the magnetizing field.

For a paramagnetic material, the magnetization vector field is collinear with the $\mathrm{H}$-field which allows the magnet field to be expressed in terms of the $\mathrm{H}$-field and the relative permeability of the fluid, $\mu_{r}$ :

$$
\vec{B}=\mu_{r} \mu_{0} \vec{H}
$$

For a non-linear magnetic material, relative permeability becomes a function of the $\mathrm{H}$-field such that $\mu_{r}=\mu_{r}(H)$. The magnetic stress tensor for an incompressible non-linear magnetic fluid is: ${ }^{55}$ 


$$
\mathbf{T}_{m}=-\left\{\mu_{0} \int_{0}^{H} M d H+\frac{1}{2} \mu_{0} H^{2}\right\} \mathbf{I}+\vec{B} \vec{H}
$$

\subsubsection{Magnetic Body Forces}

The magnetic body force density on an element of ferrofluid is equivalent to the divergence of the magnetic stress tensor:

$$
\vec{f}_{m}=\nabla \cdot \mathbf{T}_{m}=-\nabla\left\{\mu_{0} \int_{0}^{H} M d H\right\}+\mu_{0} M \nabla H
$$

Or more simply put:

$$
\vec{f}_{m}=-\mu_{0} \int_{0}^{H} \nabla_{H} M d H
$$

where $\nabla_{H} M$ is the gradient of fluid magnetization at constant $\mathrm{H}$-field strength. Recall from equations (3.1) and (3.2) that $M$ is a function of $\phi, T$, and $H$. Eq. (3.7) will be nontrivial if variations in $\phi$ or $T$ occur within the fluid. For the case of the assumed incompressible fluid with a uniform particle concentration, as long the fluid remains isothermal $(\nabla T=0)$, then $\nabla_{H} M=0$ and magnetic body forces are not present. In the event of a thermal gradient within the ferrofluid, sufficiently strong body forces can develop as to cause strong convective flows in a process known as thermomagnetic convection. ${ }^{\dagger}$ For the modeling research presented herein, magnetic body forces can be neglected since no considerable temperature gradient is present.

$\dagger$ Thermomagnetic convection can be employed to enhance heat transfer of traditional convective flows enable free convective heat transfer in micro-gravity environments where buoyancy driven (natural/free convection) flows are not possible. 


\subsubsection{Ferrofluid Interface Stress Components}

Consider the fluid interface shown in Figure 3.3, where fluid 1 and 2 have different magnetic permeabilies. Discontinuities in the magnetic components of the Maxwell stress tensor over the fluid surface results in a stress on the fluid interface.

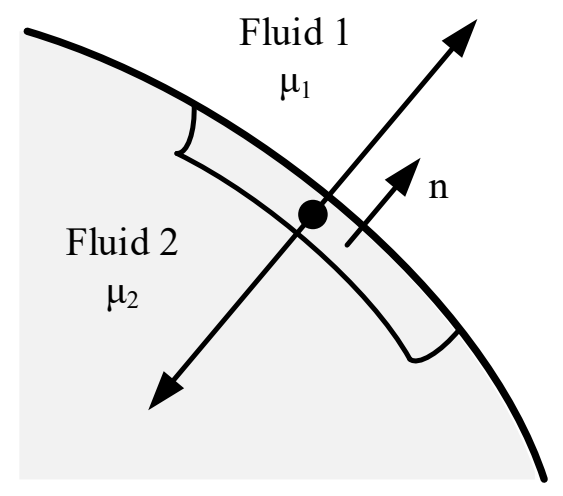

Figure 3.3: Fluid interface of ferrofluid (Fluid 2) with non-magnetic fluid (Fluid 1).

The normal and tangential components of the magnetic surface stress can be determined by differencing the magnetic stress tensor over the fluid interface.

$$
\vec{t}_{m}=\hat{n} \cdot \mathbf{T}_{m, 1}-\hat{n} \cdot \mathbf{T}_{m, 2}
$$

This stress tensor can be resolved into its respective normal and tangential components, for now, only the normal component will be investigated:

$$
\sigma_{n, \text { mag }}=\hat{n} \cdot \mathbf{T}_{m, 1} \cdot \hat{n}-\hat{n} \cdot \mathbf{T}_{m, 2} \cdot \hat{n}
$$

Substituting Eq. (3.5) into Eq. (3.9) and constraining fluid 1 to be a non-magnetic medium yields, the difference in the magnetic surface stress becomes:

$$
\sigma_{n, m a g}=\mu_{0} \int_{0}^{H} M d H+\frac{1}{2} \mu_{0}(\vec{M} \cdot \hat{n})^{2}
$$

Where $M$ and $H$ are evaluated on the side of the interface containing the magnetic fluid. A step-by-step approach for this derivation can be found in Appendix B. The term 
containing the integral is the fluid magnetic pressure. The second term in the equation is the magnetic-normal pressure. Unlike the fluid magnetic pressure, the magnetic-normal pressure exhibits saturation when the fluid magnetization becomes saturated.

When a similar approach is utilized to determine the tangential stress components, it can be found that the tangential component of the stress is equal on both sides of the fluid interface. Consequently, magnetic tangential stress do not play an active role in interface dynamics of a ferrofluid. A full proof for this relation can be found in Appendix B.

The equilibrium geometry of a fluid interface can be determined by following a similar methodology as presented in Section 2.2.2, which will yield the magnetically augmented Young-Laplace equation:

$$
\begin{aligned}
\Delta p+\frac{1}{2} \mu_{0}(\vec{M} \cdot \hat{n})^{2}+\mu_{0} \int_{0}^{H} M d H & =-\gamma\left(\nabla_{t} \cdot \hat{n}\right)-\nabla_{t} \gamma \\
& =2 \gamma R_{\text {mean }}
\end{aligned}
$$

\subsection{Normal-Field Instability}

The normal-field instability is perhaps the best-known instability demonstrated by ferrofluids. The normal-field instability forms spontaneously when a sufficiently strong magnetic field is applied perpendicular to a pool of magnetic fluid. The result is an ordered pattern of surface protuberances. This instability is a consequence of the interaction of magnetic, gravitation, and surface forces. Hydrodynamic forces should also be considered when analyzing the transition into the instability since energy alone may not capture the bifurcation and hysteresis frequently demonstrated by these fluids. ${ }^{55}$ The final pattern of the instability will be a surface topography positioned at a local minimum of the gravitation, magnetic, and capillary energies. 

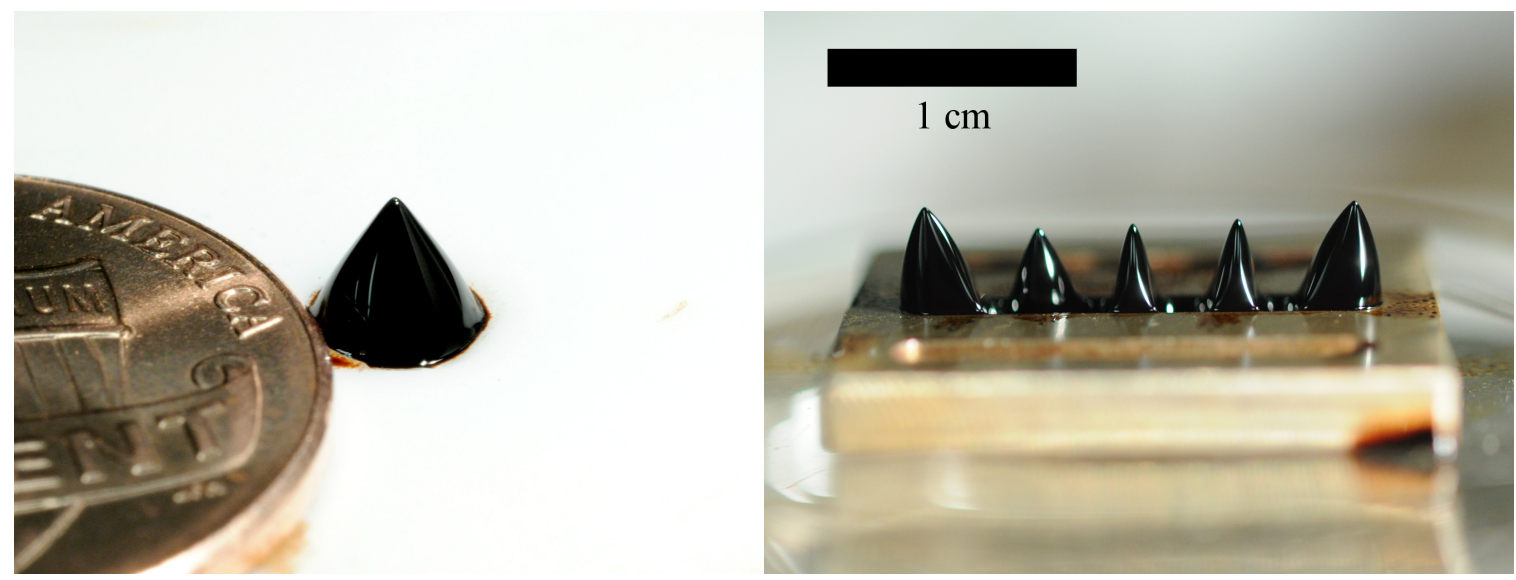

Figure 3.4: (Left) Single peak normal-field instability in a shallow reservoir. A single peak instability can be obtained for a certain ranges of field strengths and fluid volumes if the fluid is prevented from spreading. The fluid can be constrained by imposing a boundary or infusing the droplet within a uniform field. (Right) Multipeak instability in a shallow-long reservoir.

An analytical analysis for this instability was first presented by Cowley and Rosensweig in $1967 .{ }^{69}$ Subsequent publications have also presented analytical investigations on the instability; however, all have focused on the critical magnetic field value to initiate the instability,or attempts to predict what instability mode will dominate to predict the instability pattern. ${ }^{70-73}$ Because of the complexity inherent to instabilities, more advanced analyses of the normal-field instability require numerical means

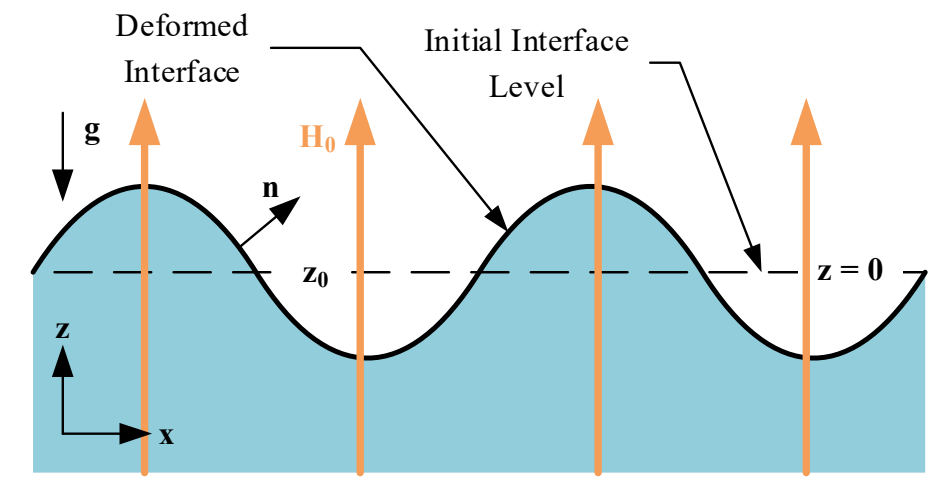

Figure 3.5: Perturbed ferrofluid interface resulting from a uniform magnetic field. The interface will remain flat along $z_{0}$ until a critical field is applied. Diagram adapted from (Rosensweig 1997). ${ }^{55}$

In the absence of any magnetic field, a pool of ferrofluid will lie completely flat. As the strength of the applied field increases, the ferrofluid surface will remain relatively undisturbed, forming only a slight plateau, until the ferrofluid reaches a critical 
magnetization $M_{c}$. Rosensweig performed a stability analysis and found the critical magnetization for non-linear ferrofluids to be: $:^{55}$

$$
M_{c}^{2}=\frac{2}{\mu_{0}}\left(1+\sqrt{\frac{\mu_{0}^{2}}{(B / H)_{0}(\partial B / \partial H)_{0}}}\right)(g \Delta \rho \sigma)^{1 / 2}
$$

Once the critical fluid magnetization is reached, the conditions for the normal-field instability are met and the fluid will transform into a pattern of peaks.

To better understand why a pool of ferrofluid forms the array of peaks and valleys in the presence of a magnetic field, it is advantageous to consider the phenomenon from an energy standpoint. The interface can again be described as a balance of energies which must now include the magnetic energy. The interface function, $z=z(x, y)$, for the disturbed surface will minimize the system energy, $U(z)$, which is composed of individual energy components: ${ }^{55}$

$$
U(z)=U_{g}+U_{s}+U_{m}
$$

Whereby the gravitational energy is:

$$
U_{g}=\frac{1}{2} \rho g \iint z^{2}(x, y) d x d y
$$

The surface energy is:

$$
U_{s}=\iint \gamma\left[1+(\partial z / \partial x)^{2}+(\partial z / \partial y)^{2}\right]^{1 / 2} d x d y
$$

And the magnetic-field energy is:

$$
U_{m}=\oiiint_{d V} \int H d B d V
$$


The magnetic stress exerts an upward motion on the fluid - increasing the magnetic energy and further increasing the magnetic stress. However, such a trend is bounded since the surface deformation increases the surface energy which limits further growth. The pattern of peaks and valleys observed is simply a minimum energy solution for the fluid when in equilibrium.

Since a large pool of ferrofluid is assumed for this analysis, contact line energy is not considered, which should be considered for smaller pools of ferrofluid. It is worth noting, a purely energy-based approach should be used with caution since that approach does not incorporate the fluid motion. Simply put, a purely energy approach could find a global minimum solution to the equilibrium balance which cannot be reached physically due to the fluid physics. 


\section{Chapter 4 Modeling Interface Deformation and Spray Onset of a Ferrofluid Meniscus}

When electrospray emission is achieved from an electrically conductive ferrofluid in the presence of a magnetic field, the electric stresses and magnetic stresses act in tandem to stretch the fluid meniscus. However, the relative influence of the magnetic stresses on this process remains uncertain. It is hypothesized that for weak electric fields, the magnetic stresses dominate - controlling the meniscus geometry. As the applied voltage increases, the electric stresses grow off the perturbation resulting from the magnetic stresses eventually growing to dominate near onset. The aim of the research presented in this chapter was to develop a modeling tool to predict the deformation of a fluid interface under simultaneous electric and magnetic stresses. Such a tool will be instrumental in analyzing the dynamics leading up to spray emission.

The precise voltage needed to achieve emission is referred to as onset voltage, $V_{\text {start }}$. Several models have been developed to predict the onset voltage of electrospray. However, these models do not contain magnetic stresses and impose a fluid geometry in varying degrees. The most eminent of these models is by Prewett \& Mair. ${ }^{74}$ In this model onset voltage for electrospray is predicted as the condition when the apex electric stress equals the capillary surface stress; assuming the meniscus is a hyperboloid having tip radius $R_{c}$ separated from the extractor electrode by a distance $d$. When $d \gg R_{c}$, the Prewett \& Mair approximation becomes: ${ }^{74}$ 


$$
V_{\text {start }}=\ln \left(\frac{2 d}{R_{c}}\right) \sqrt{\frac{\gamma R_{c}}{\varepsilon_{0}}}
$$

While this relationship is intuitively straightforward, it is only an approximation and is not physically rigorous. The premise of Prewett \& Mair is that the fluid will emit spray when the electric traction on the interface exceeds the surface tension. In reality, this condition will never occur; the capillary stress depends upon the coefficient of surface tension and the interface radius of curvature. In response to increasing electric stress the pliant meniscus will sharpen without limit (radius of curvature $r$ can decrease to zero) and thus there is no practical upper bound on the capillary stress that can be 'exceeded' by the electric stress. Despite this, for many instances the Prewett \& Mair relationship agrees adequately with observations for traditional electrospray in select cases - typically when some external structure such as a hollow capillary or externally wetted needle is present to impose a geometric curvature length scale independent of the fluid properties.

Magnetic stresses are not included or anticipated in the onset model of Prewett \& Mair. The derivation of this model assumes a hyperboloid geometry. Assuming the surface of the fluid to be equipotential, the electric field outside can thus be found analytically. The formulation simply does not permit a magnetic field to be found.

Another electric-field-only onset model was developed by Krpoun and Shea. ${ }^{75}$ This approach modeled the fluid deformation leading up to onset using a combined finite element model for the stresses and an analytical model for the geometry. Unlike the Prewett \& Mair approach the technique of Krpoun and Shea includes a deformable meniscus, however the meniscus shape is constrained to be a conic section defined by a BernsteinBezier curve and thus the self-consistent meniscus profile is not obtained in the process of solution. This model can easily to be adapted to model a magnetically responsive fluid and calculate interface stresses. Unfortunately, the lack of a self-consistent interface prevents an accurate analysis of the role of magnetic stresses in preconditioning the electrified meniscus.

For an accurate analysis of the complementary role of the magnetic and electric stresses on the fluid meniscus, it became evident that a first principles approach is necessary which 
permits the meniscus to arbitrarily deform. A review of the literature reveals that the deformation of a fluid meniscus under magnetic or electric stresses have focused primarily on suspended droplets in the presence of a single field (either E or H) ${ }^{76-81}$ with much less attention given to the case when both fields are present. ${ }^{82,83}$ When the fluid droplet is pinned to a surface, the shape of the droplet is complicated by the addition of the contact surface energy. Previous investigation into the deformation of sessile droplets under the influence of either electric or magnetic fields has been studied numerically using finite element methods, ${ }^{84-89}$ but little attention has been given to the meniscus behavior of a sessile droplet under the combined action of electric and magnetic stresses.

\subsection{Goals of Study}

The overarching goal of this research was to understand the inter-relation between magnetic, electric, and surface tension stresses during the run-up to spraying instability of an ionic liquid ferrofluid. The problem was approached by observing the onset of instability through laboratory testing and also analyzing the laboratory configurations using a dynamic fluid/electromagnetic simulation. Two configurations were analyzed: the first configuration was the meniscus formed at the tip of a hollow capillary needle, and the second configuration was a sessile droplet on a flat plate. Both configurations were subjected to controlled electric and magnetic fields introduced via biased electrodes and a Helmholtz coil.

In the following sections, the fluid properties and constitutive equations describing ferrofluid magnetization are presented.

\subsection{Fluid Properties}

The ferrofluid utilized for this study was an ionic liquid based ferrofluid. The carrier fluid was a EMIM-NTf2 and the nanoparticles were suspended with a polymer coating. This ferrofluid was produced by the Key Centre for Polymers and Colloids at the University of Sydney. The fluid properties are presented below in Table 4-1: 
Table 4-1: Ferrofluid fluid and magnetic properties

\begin{tabular}{|c|c|c|c|}
\hline & & & $\begin{array}{l}\text { EMIM-NTf2 } \\
\text { ILFF }^{34,62, ~ 90-92}\end{array}$ \\
\hline \multicolumn{2}{|c|}{ Carrier Fluid } & & EMIM-NTf2 \\
\hline \multicolumn{2}{|l|}{ Density } & $\left(\mathrm{g} / \mathrm{cm}^{3}\right)$ & 1.815 \\
\hline \multicolumn{2}{|l|}{ Viscosity } & $(\mathrm{mPa} \cdot \mathrm{s})$ & 120 \\
\hline \multicolumn{2}{|c|}{ Surface Tension } & $(\mathrm{mN} / \mathrm{m})$ & 32.389 \\
\hline \multicolumn{2}{|c|}{ Conductivity } & $(\mathrm{S} / \mathrm{m})$ & 0.63 \\
\hline \multicolumn{2}{|c|}{ Percent Solids } & wt. \% & $17.3-20.6$ \\
\hline \multicolumn{2}{|c|}{ Particle Size } & $(\mathrm{nm})$ & $\sim 10-15$ \\
\hline \multirow{3}{*}{$\begin{array}{c}\text { Langevin } \\
\text { Fit }\end{array}$} & $\beta$ & $(\mathrm{A} / \mathrm{m})$ & $2.335 \times 10^{4}$ \\
\hline & $\tau$ & $(\mathrm{m} / \mathrm{A})$ & $2.408 \times 10^{-4}$ \\
\hline & $R^{2}$ & & 0.959 \\
\hline
\end{tabular}

The magnetization data for the fluid was measured using a vibrating sample magnetometer and was provided by the University of Sydney. The Langevin expression presented in Eq. (3.1) was fit to the magnetization data in terms of the parameters, $\beta$ and $\tau$ :

$$
M=\beta\left[\operatorname{coth}(\tau|H|)-\frac{1}{\tau|H|}\right]
$$

where $\tau H$ is the ratio of the magnetic to kinetic energy of each nanoparticle and $\beta$ is a function of the bulk magnetization of the nanoparticle material and the volume fraction of the particles in the ferrofluid. The relative permeability as a function of $H$ thus becomes:

$$
\mu_{r}=\frac{M+H}{H} \Rightarrow \mu_{r}=1+\frac{\beta}{H}\left[\operatorname{coth}(\tau|H|)-\frac{1}{\tau|H|}\right]
$$

A Langevin fit of the ionic liquid ferrofluid magnetization data is presented in Figure 4.1: 


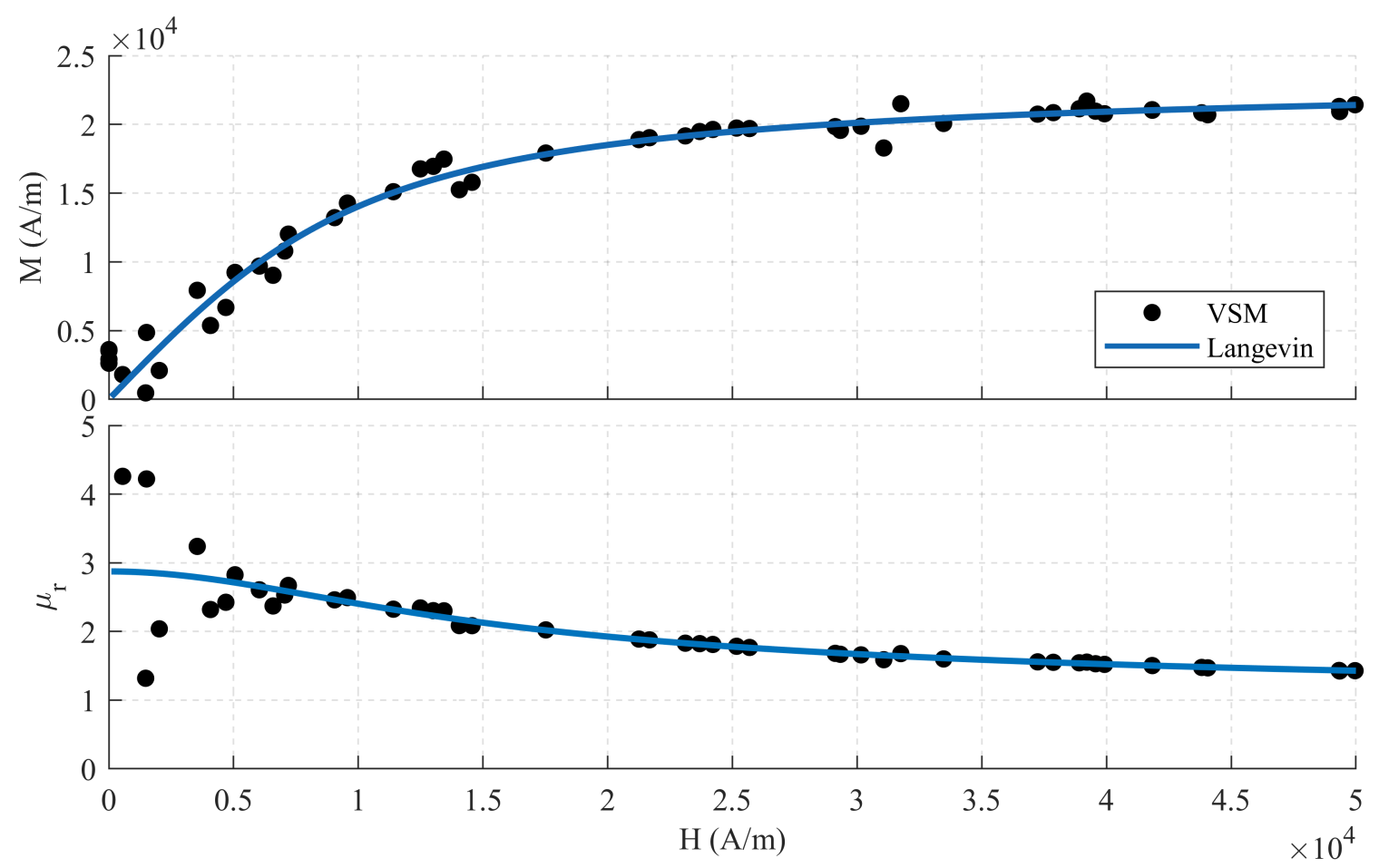

Figure 4.1: Magnetization and relative permeability of the ionic liquid ferrofluid (Data obtained from combining magnetization data from batches: NJ397053, NJ397074, and NJ397091). Magnetization data, which was provided by the University of Sydney, was measured using a vibrating sample magnetometer.

\subsection{Capillary Needle Electrospray Emitter}

Electrospray from a hollow capillary needle has been studied at great depth in the literature. To provide a baseline comparison, the first objective was to observe how the addition of magnetic stress changes the well documented onset of capillary electrospray. Ferrofluid was contained within a capillary needle biased to a high voltage with a grounded electrode in proximity. The needle fixed the base of the fluid meniscus, making the contact line independent of the strength of any applied field. This geometry enables a direct comparison between onset with and without a magnetic field that cannot be obtained with a sessile droplet. 


\subsubsection{Experimental Setup*}

The needle emitter, shown in Figure 4.2, was positioned within a Helmholtz coil capable of generating a magnetic field of $200 \mathrm{G}$.

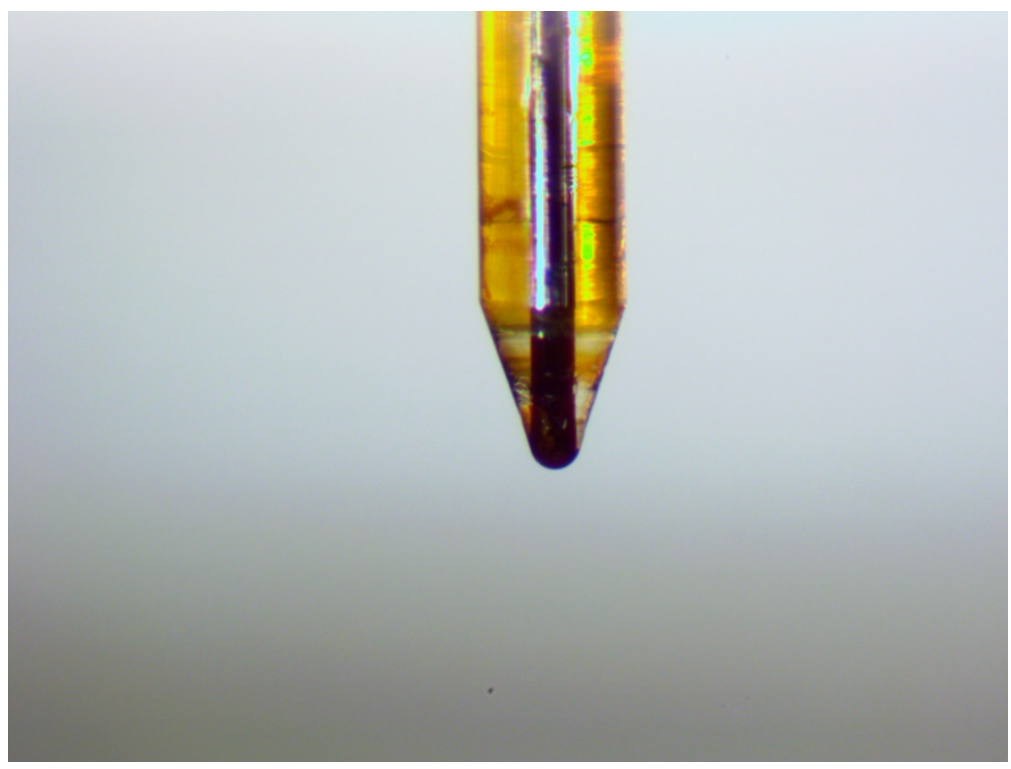

Figure 4.2: Electrospray capillary needle with ILFF. Needle biased with respect to a grounded collector plate (not shown) separated from the needle tip by $1.32 \mathrm{~mm}$. Dimensions: ID $=75 \mu \mathrm{m}, \mathrm{OD}=360 \mu \mathrm{m}$.

The emission source was insulated from the heat generated by the coil through the use of multi-layer insulation. A glass vial, containing a fluid reserve, was connected to the emission source via a continuous glass capillary. This vial was positioned above the needle tip such that hydrostatic pressure would form a meniscus at the needle exit. The fluid was biased with respect to a grounded extraction electrode. Emission current was measured using an ammeter placed between the power supply and the emitter. The needle and extraction electrode were separated by $1.32 \mathrm{~mm}$ and the needle inner diameter was $75 \mu \mathrm{m}$. A diagram of this experimental setup is presented in Figure 4.3.

\footnotetext{
* The experimental portion of the ILFF needle onset study entailed within this section was performed by Kurt J. Terhune.
} 


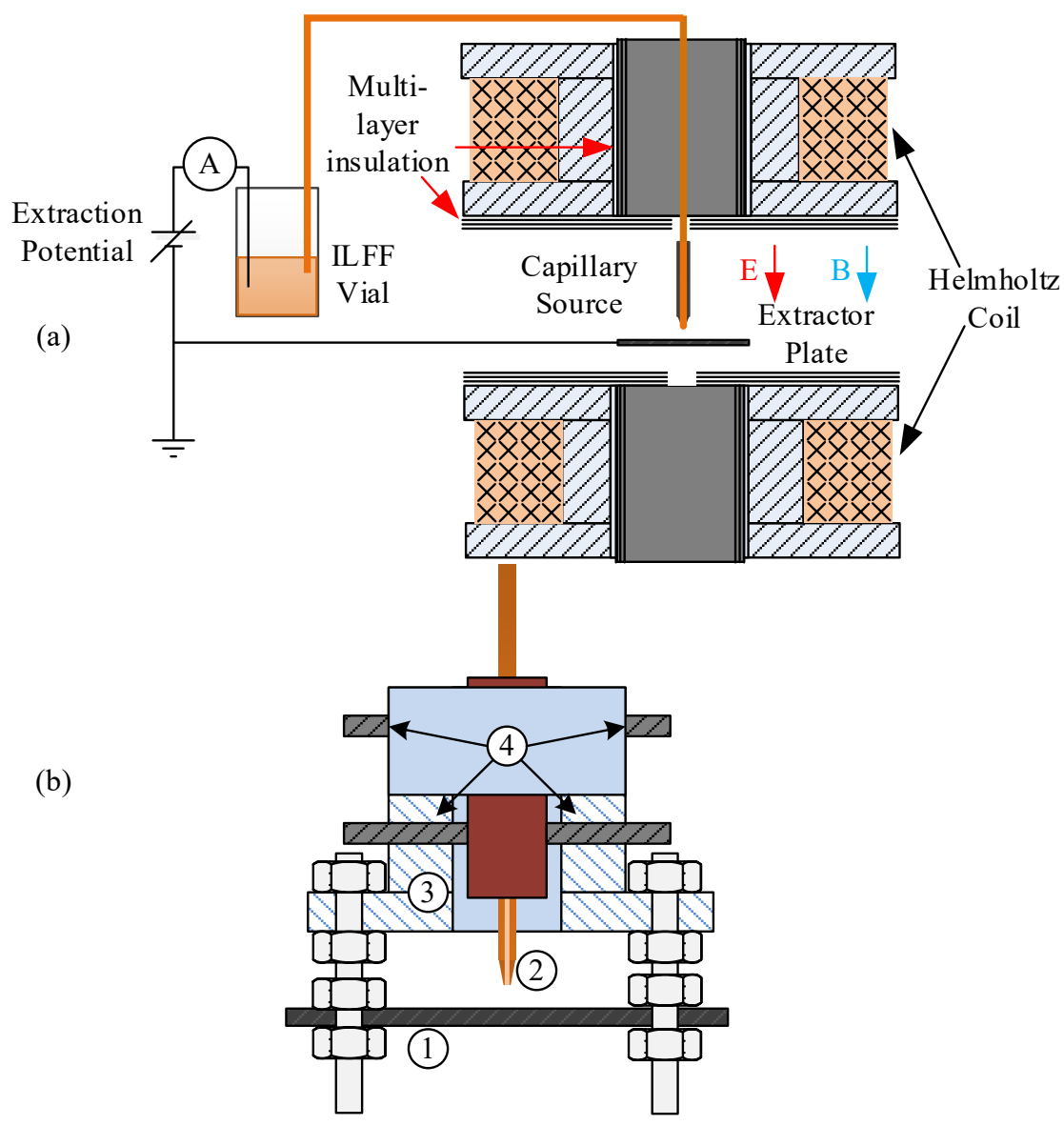

Figure 4.3: (a) ILFF capillary needle emitter in Helmholtz coil setup. (b) Needle emitter holder: (1) extractor plate, (2) capillary needle, (3) PTFE isolation block, and (4) alignment set screws. (Illustration created by K. J. Terhune with modifications made by the author for this dissertation.)

\subsubsection{Computational Approach}

The coupled electromagnetics and fluid mechanics of the capillary needle experiment were modeled using COMSOL. The computational domain for this study is presented in Figure 4.4. The two-phase flow interface was applied to region 1, outside this region fluid motion will be minimal and will have negligible influence on interfacial dynamics. This region was therefore treated as a static medium to reduce the computational load. Inflow and outflow boundary conditions were included in region 1 to permit ferrofluid to enter and displaced air to leave the region. The electrostatics and magnetostatics interfaces were applied to all domains. 


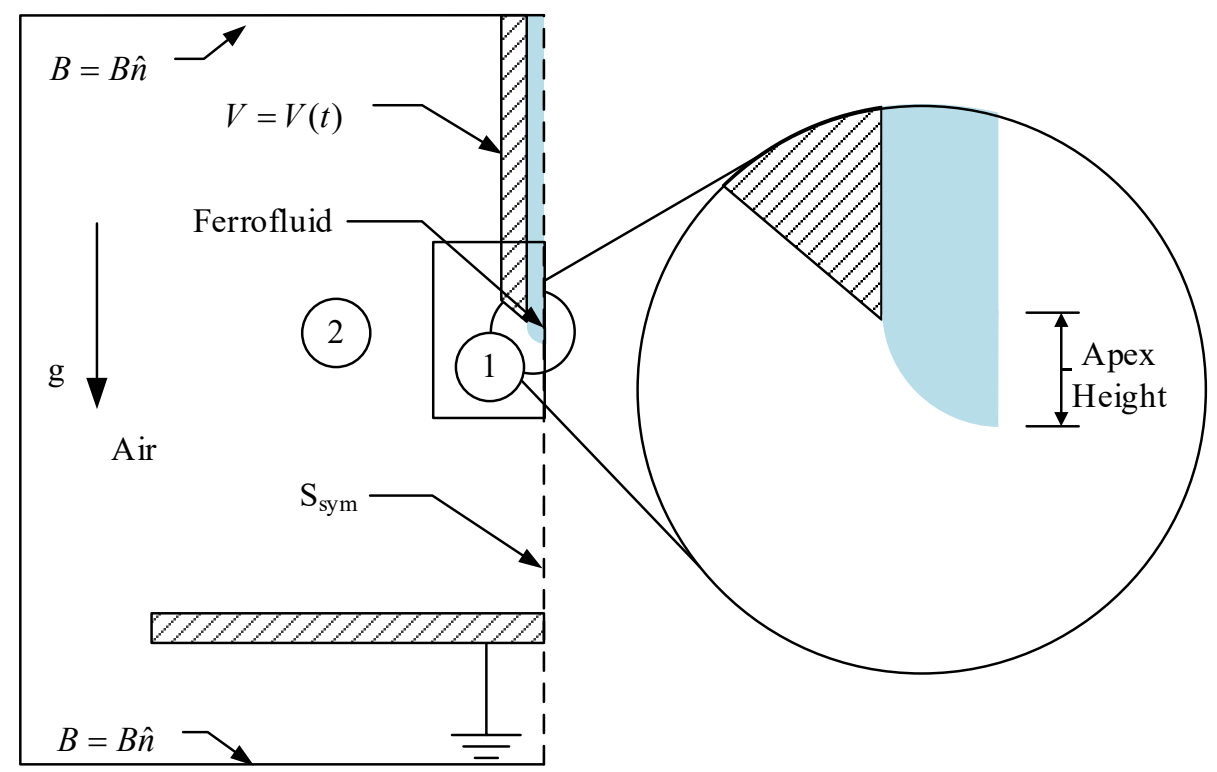

Figure 4.4: Simulation domain for a ferrofluid-filled capillary needle subject to an aligned magnetic field. Fluid dynamics were solved in region (1) while the fluid was assumed to be a static in region (2).

The fluid deformation was modeled using the two-phase flow moving mesh physics module. This module is based on the Arbitrary Lagrangian-Eulerian technique - a method which allows interfacial nodes to move to accurately model the ferrofluid-air interface while the interior nodes move to optimize element quality. This physics interface modeled the fluid using the following incompressible laminar flow version of the Navier-Stokes equation:

$$
\begin{gathered}
\rho \frac{\partial \vec{u}}{\partial t}+\rho(\vec{u} \cdot \nabla) \vec{u}=\nabla \cdot\left[-p \mathbf{I}+\mu\left(\nabla \vec{u}+(\nabla \vec{u})^{T}\right)\right]+\rho \vec{g}_{0} \\
\rho \nabla \cdot \vec{u}=0
\end{gathered}
$$

The fluid-fluid interfacial stress balance presented in the following stress balance:

$$
\hat{n} \cdot \mathbf{T}_{1}-\hat{n} \cdot \mathbf{T}_{2}=\gamma\left(\nabla_{t} \cdot \hat{n}\right) \hat{n}-\nabla_{t} \gamma
$$

was solved along the fluid interface with contributions from the following three equations:

$$
\text { Stokes Tensor: } \quad \mathbf{T}_{s}=-p \mathbf{I}+\mu\left[\nabla \vec{u}+(\nabla \vec{u})^{T}\right]
$$




$$
\begin{array}{lc}
\text { Magnetic Tensor: } & \mathbf{T}_{m}=-\left\{\mu_{0} \int_{0}^{H} M d H+\frac{1}{2} \mu_{0} H^{2}\right\} \mathbf{I}+\vec{B} \vec{H} \\
\text { Electric Tensor: } & \mathbf{T}_{e}=-\left\{\frac{1}{2} \varepsilon E^{2}\right\} \mathbf{I}+\vec{D} \vec{E}
\end{array}
$$

The mesh velocity along the interface was subject to the following constraint:

$$
\vec{u}_{\text {mesh }}=\left(\vec{u}_{1} \cdot \hat{n}_{1}\right)
$$

The magnetic field was solved using the magnetostatics module in terms of the magnetic potential $V_{m}$. In a static domain where there is no electric current, the magnetic field is related to the magnetic potential as follows:

$$
\vec{H}=-\nabla V_{m}
$$

From Gauss's law for magnetism, $\nabla \cdot \vec{B}=0$, the following relation can be derived, which is solved within the simulation domain in conjunction with Eq. (4.11):

$$
\nabla \cdot\left(\mu_{0} \mu_{r} \vec{H}\right)=0
$$

The relative permeability, $\mu_{r}$, is found using the constitutive equation presented in Eq. (4.3). The following boundary condition is applied to the fluid-air interface to ensure continuity of the normal component of the magnetic field:

$$
\hat{n} \cdot\left[\left(\mu_{0} \mu_{r} \vec{H}\right)_{1}-\left(\mu_{0} \mu_{r} \vec{H}\right)_{2}\right]=\hat{n} \cdot\left(\vec{B}_{1}-\vec{B}_{2}\right)=0
$$

The electric field was solved using the electrostatics module in terms of the electric potential $V$ :

$$
\vec{E}=-\nabla V
$$


Subject to:

$$
\nabla \cdot \vec{D}=0
$$

where $\vec{D}=\varepsilon \vec{E}$. The coupling of the electric and magnetic stress tensors with the fluid stress tensor was achieved with the use of a weak form contribution in the following form:

$$
\left(\sigma_{e}+\sigma_{m}\right)[\operatorname{test}(u) \cdot n r+\operatorname{test}(u) \cdot n z]
$$

Where test () is the Test Function (determined by COMSOL) and $\sigma_{e}$ and $\sigma_{m}$ are the normal electric and magnetic stress components. Modeling the fluid as a perfect electrical conductor, the normal electric stress becomes:

$$
\begin{aligned}
\sigma_{e}^{n} & =\left(\hat{n} \cdot \mathbf{T}_{e}^{+} \cdot \hat{n}\right)-\left(\hat{n} \cdot \mathbf{T}_{e}^{-} \cdot \hat{n}\right) \\
& =\frac{1}{2} \varepsilon_{0} E_{n}^{2}
\end{aligned}
$$

while the normal component of the magnetic stress is:

$$
\begin{aligned}
\sigma_{m}^{n} & =\left(\hat{n} \cdot \mathbf{T}_{m}^{+} \cdot \hat{n}\right)-\left(\hat{n} \cdot \mathbf{T}_{m}^{-} \cdot \hat{n}\right) \\
& =\mu_{0} \int_{0}^{H} M d H+\frac{1}{2} \mu_{0} M_{n}^{2}
\end{aligned}
$$

Note: $\mathbf{T}_{e}$ and $\mathbf{T}_{m}$ are the electric and magnetic stress tensors and the superscripts + and - denote the fluid above and below the ferrofluid-air interface, respectively. Due to the high conductivity of the ionic liquid carrier fluid, the ferrofluid was modeled as a perfect electrical conductor with an equipotential fluid interface. For a perfect dielectric or perfect conductor, no tangential electric surface stress is present. Boundary conditions on $B_{n}$ and $H_{t}$ over the fluid interface preclude a tangential magnetic stress. Derivations of Eq. (4.17) and (4.18) as well proof of the absence of corresponding tangential stresses are presented 
in detail in Appendices A and B. The Helmholtz field was applied as a boundary condition far from the fluid volume where the contribution to the field from the fluid is negligible. At each solver step, the instantaneous fluid geometry was used to calculate the local magnetic and electric fields.

A mesh validation study was performed with element densities of 350,700, 1000, and 1500 along the fluid interface for the sharpest peaks investigated. The distribution scheme employed concentrated elements at the apex - growing the elements gradually along the interface towards the base. The refinement study revealed less than a $0.1 \%$ variation in apex height over the meshes investigated, and thus the baseline 350 elements was selected to reduce the computational cost. Adaptive remeshing was integrated into the model such that when the minimum element quality became less than 0.1 (a minimum recommended by the software package), the geometry was remeshed. This enabled large deformations in the geometry. Droplet volume and the element count along the fluid interface were conserved during the remeshing process. Element quality was based on the skew factor of the triangular elements, and the average mesh quality in the domain was always between 0.97 and 0.98 .

A dynamic simulation was employed that is in principle capable of providing the temporal response of the fluid. However, the goal was to recover the static solutions and so the dynamic simulation was used in the following manner. The meniscus was initially defined as a hemisphere and the magnetic field was set to a constant value. The dynamic simulation then computed the shape relaxation of the hemispherical meniscus to the final (non-hemispheric) static profile governed by the magnetic stress. The simulation ran for 0.2 seconds, although the droplet profile typically did not evolve any measurable amount after 0.1 seconds. The interim dynamic response of the meniscus was of little relevance to our study and thus no information is reported here other than final static configurations. The effect of electric stress was then computed by starting a new simulation that used the previously converged meniscus profile as an initial condition. In this new simulation, the extraction voltage was quickly ramped up to a pre-set value and then held constant for a long-time period compared to the meniscus dynamic response to this changing voltage. Within 0.1 seconds, the meniscus converged to a new steady-state shape governed by the 
magnetic and electric stresses. This process was repeated for a number of different voltages and magnetic fields in order to assemble static results as a collection of multiple dynamic simulations. This process is presented below in Figure 4.5 while the instantaneous mesh for four snapshots in time is presented in Figure 4.6.

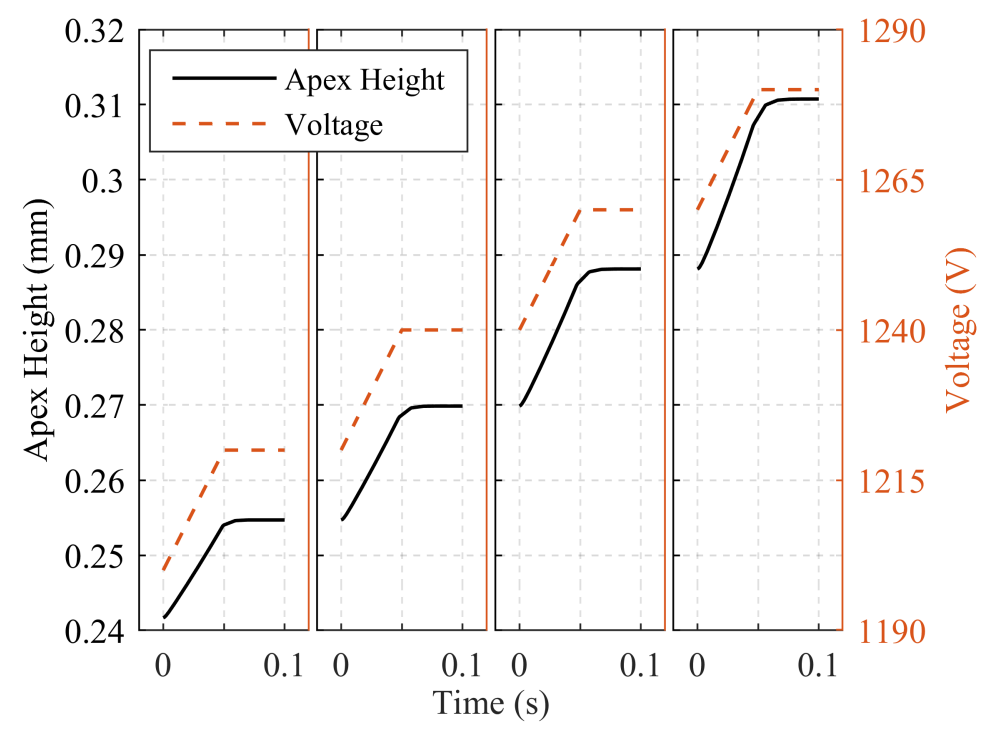

Figure 4.5: Static meniscus profiles are obtained as a collection of individual dynamic simulations. For each solution step, the previously solved meniscus profile was used as an initial condition for a new dynamic simulation. The applied voltage was ramped from the previous value to the new value over 0.05 seconds and the meniscus was allowed to dynamically respond to the new conditions. The final steady-state result is reported as the static solution. 


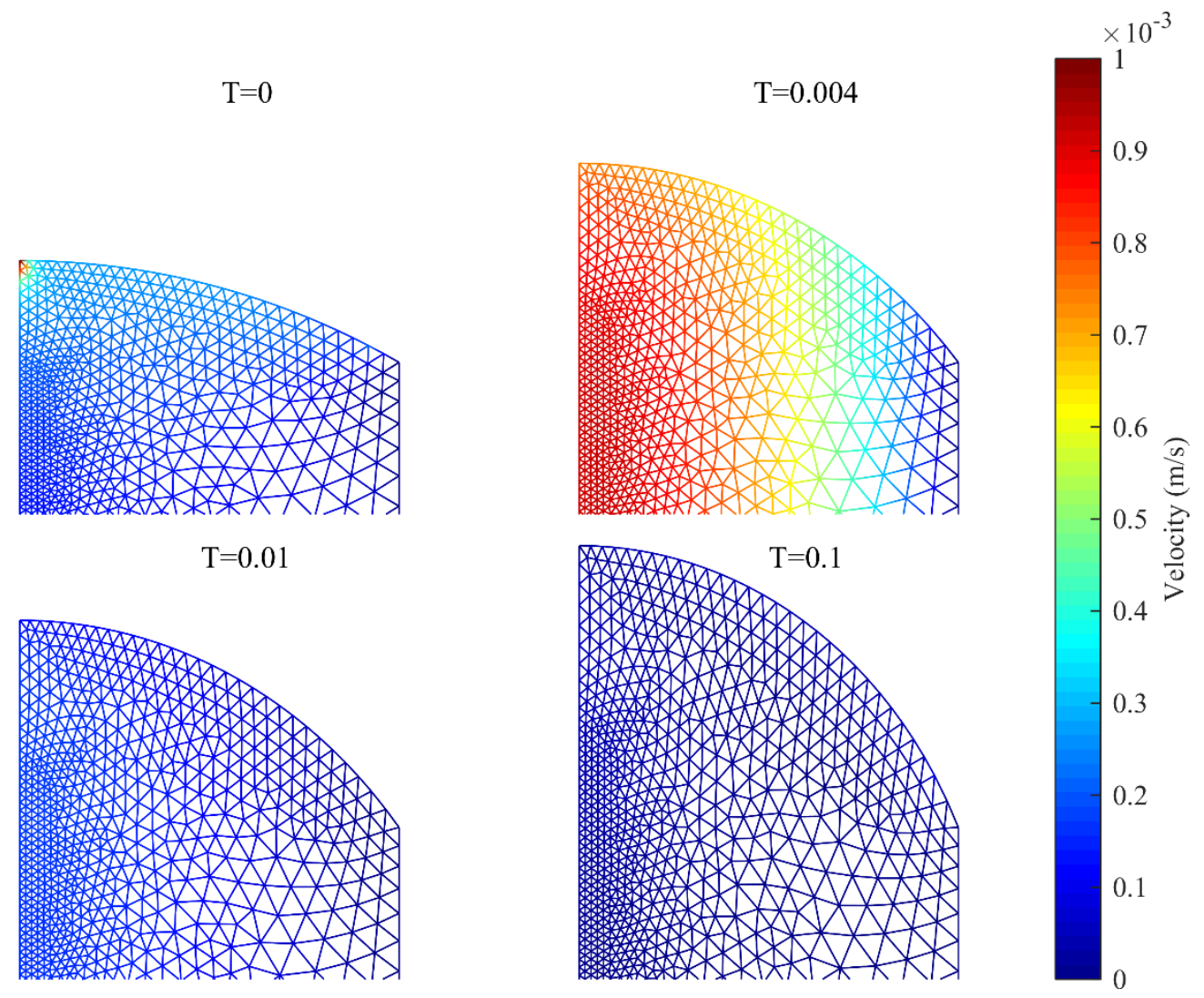

Figure 4.6: Capillary needle simulation mesh. Electric field ramped over 0.01 seconds. Mesh elements will deform to track fluid movement. Element density reduced from level used in presented results to enhance visibility. Simulation results were taken at 0.1 seconds, when the meniscus reached equilibrium.

\subsubsection{Simulation Results and Comparison with Experiment}

Both the applied electric field and the applied magnetic field exert stress on the meniscus such that the convex profile is elongated in the direction of the field while the base remains pinned to the capillary exit. The COMSOL model was used to calculate the meniscus shape and from this the "apex height," which is the dimension from the terminal position of the apex to the capillary exit. Simulation results are presented in two forms. In Figure 4.7, the apex height of the meniscus is presented against extraction voltage. In Figure 4.8, the height of the meniscus is normalized by the inner capillary radius which is then plotted in terms of the following Bond numbers:

$$
\text { Electric Bond Number } \quad B_{e}=\varepsilon_{0} E_{a}^{2} R_{0} / \gamma
$$


Magnetic Bond Number $\quad B_{m}=\mu_{0} H_{0}^{2} R_{0} / \gamma$

In the previous equations, $E_{a}$ and $H_{0}$ are the apex electric field and free space (Helmholtz coil without presence of fluid) magnetizing field, respectively. $R_{0}$ is the interior radius of the capillary needle, and $Z$ is the apex height. For a given voltage the apex height increases with the strength of the applied magnetic field, which acts in tandem with the electric stress to contribute to the deformation of the meniscus. As the stress is increased the meniscus transitions into an instability which results in spray emission. The simulation is not capable of predicting the fluid behavior while spraying, however the runup to spray onset is evidenced by the asymptotic growth in apex height. Onset was approximated by the highest voltage at which a stable solution could be obtained, where the voltage was increased incrementally by 1 volt until solution was no longer possible.

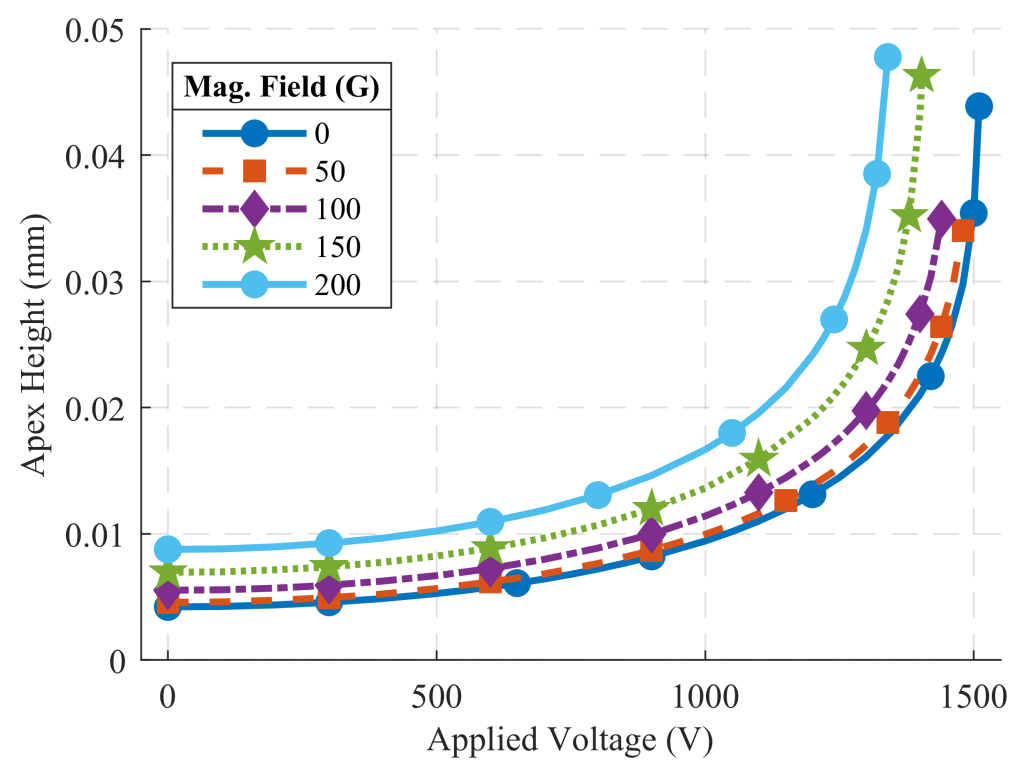

Figure 4.7: Simulated apex height vs applied potential of the capillary needle meniscus for five magnetic field strengths. 


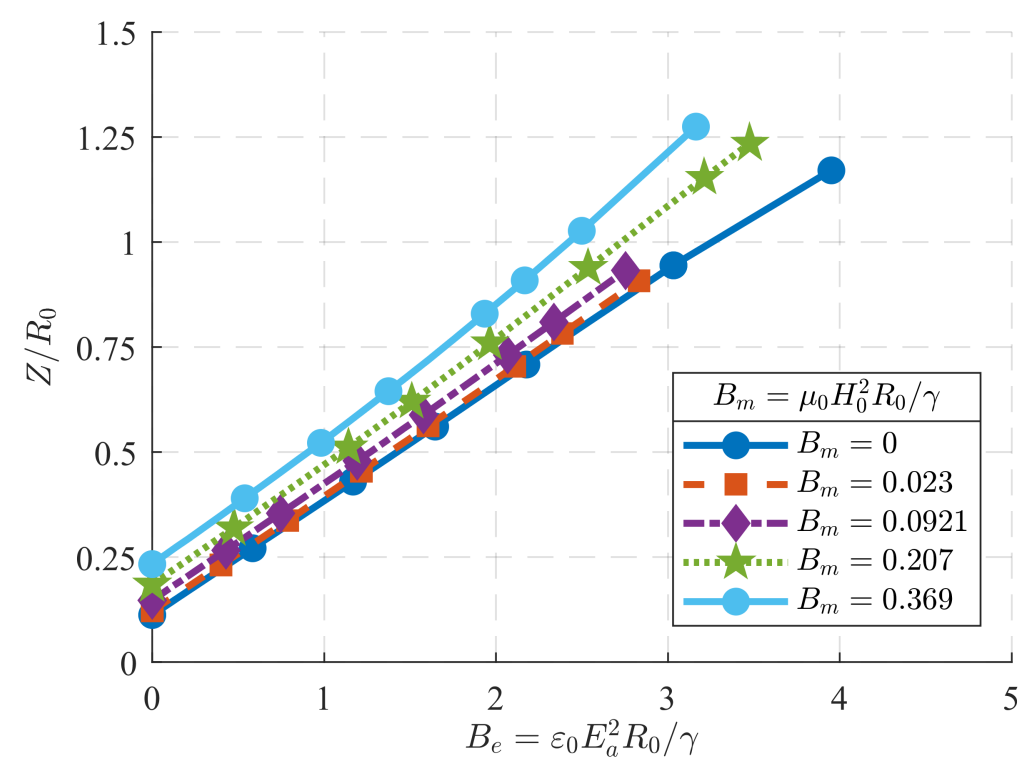

Figure 4.8: Scaled apex height using electric and magnetic Bond numbers.

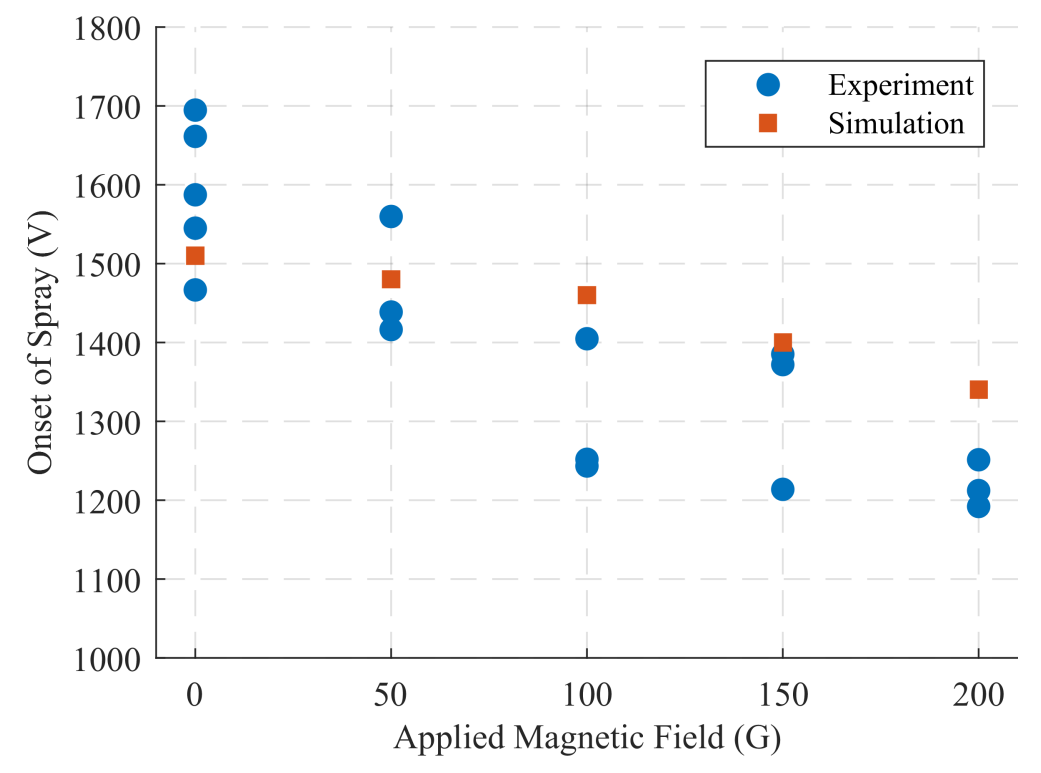

Figure 4.9: Experimental and simulated onset of emission potential or the capillary emitter. Simulated onset was approximated as the highest voltage at which a stable solution could be obtained when approaching from below by 1-volt increments.

Spray onset was easily observed in the laboratory experiment by the sudden appearance of emission current as the bias voltage was slowly increased on the capillary. Three startup tests were performed for each magnetic field strength and the test-to-test variation in observed onset voltage was used to establish uncertainty bars. The experimentally measured and simulated onset voltages for the capillary needle are compared in Figure 4.9. 
In the case of the experimental results, a $200-\mathrm{G}$ magnetic field resulted in a $22 \%$ drop in onset potential. Overall, the model performed well at predicting the onset of emission of the capillary needle emitter for the range of magnetic fields studied. It is instructive to use the model to analyze the relative effects of electric and magnetic stress. Figure 4.10 shows the two components of stress for the $200-\mathrm{G}$ magnetic field test case. Near the onset of spray the electric stress at the meniscus apex is five to ten times larger than the magnetic stress for the highest obtainable stable solution. The primary role of the magnetic stress is to precondition the meniscus shape such that threshold to instability occurs at a significantly lower voltage.

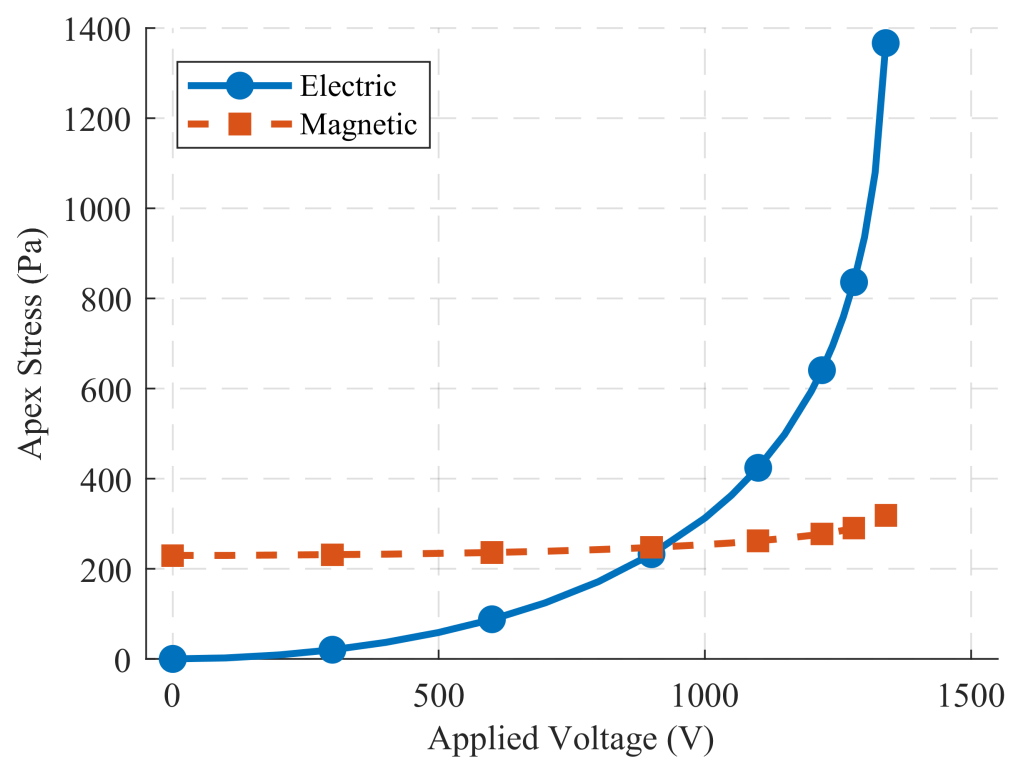

Figure 4.10: Magnetic and Electric pressures at the meniscus apex for increasing applied voltage for a magnetic field of $200 \mathrm{G}$.

\subsection{Predictive Model of Electromagnetic Sessile Droplet Deformation}

The motivation for this research was to understand how electric and magnetic stress act together to cause spray from the fluid tips formed via the normal-field instability. The capillary needle experiment and simulation reported in Section V was used to develop and validate a predictive modeling tool. With the foundations of this tool in hand, the second objective was to remove the capillary structure and use the model to predict fluid motion 
and interface dynamics for an arbitrary droplet and field configuration. The model was further develop and validate the model through computational and experimental studies of an electric and magnetically stressed sessile droplet.

\subsubsection{Computational Approach}

The dynamic simulation was designed to solve for temporal meniscus evolution and equilibrium steady-state geometry for arbitrary fields. The computational domain is shown in Figure 4.11. The meshing, adaptive remeshing, and simulation approach for this study was identical to the approach utilized in Section 4.3.2 for the capillary needle. To reduce the computational load, the physics interfaces were solved only in the necessary domains. The location of the pinning point (denoted by $R$ in Figure 4.11) was determined by imaging the sessile droplet in the absence of any electric field. The initial fluid geometry within the model was set to have an equivalent volume and contact-plane radius as the imaged droplet. No constrains were placed on the contact angle. A sample of the mesh evolution is presented in Figure 4.12.

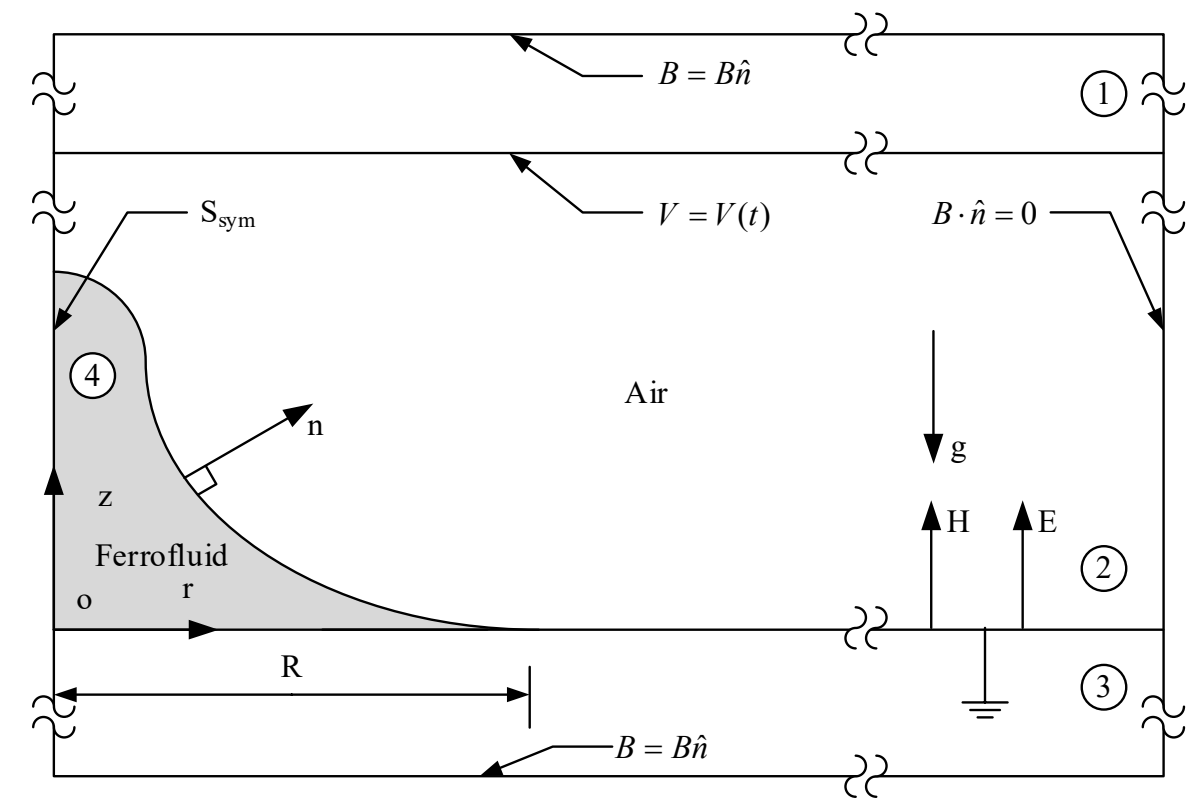

Figure 4.11: Simulation domain for single ferrofluid droplet subject to a vertical magnetic and electric field. 


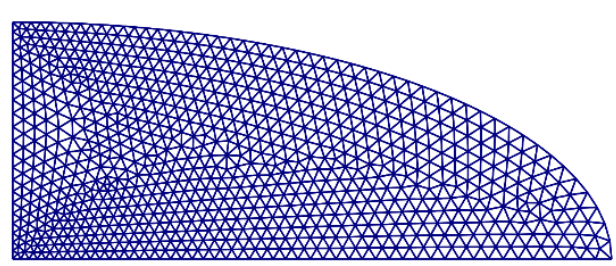

$\mathrm{T}=0.09$

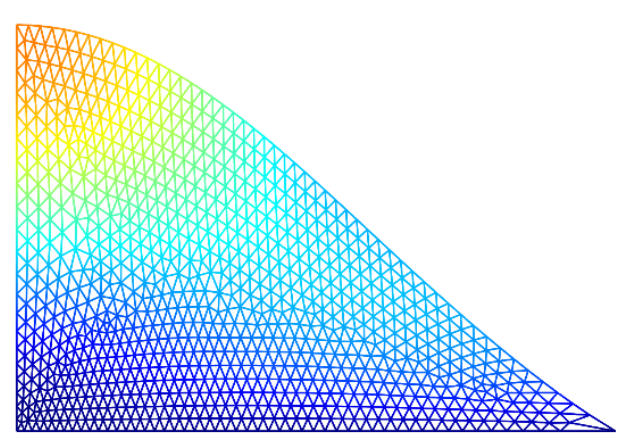

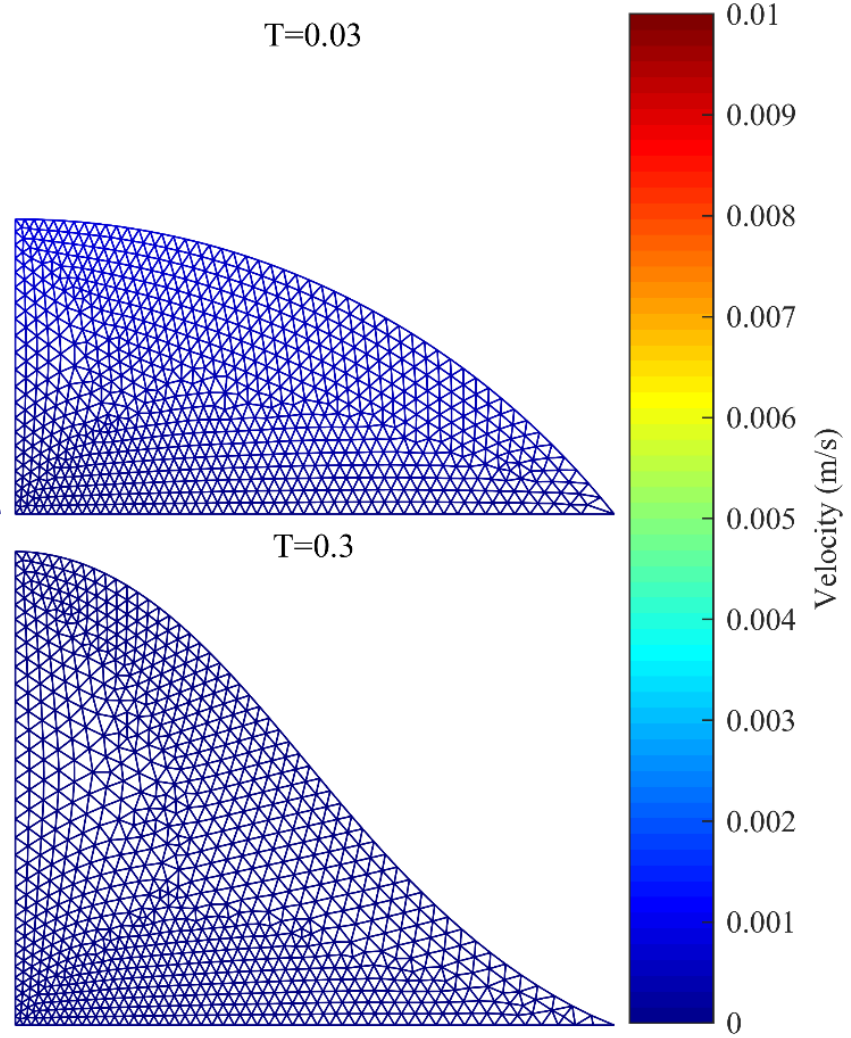

Figure 4.12: Sessile droplet mesh. Magnetic field ramped over 0.1 seconds. Mesh elements will deform to track fluid movement. Element density reduced from level used in presented results to enhance visibility. Simulation results were taken at 0.5 seconds, while the mesh reached steady state around 0.3 seconds.

\subsubsection{Experimental Setup}

An imaging apparatus was utilized to capture the shape of a sessile ferrofluid droplet under controllable electric and magnetic fields. This method of imaging allows for precise edge detection. The images setup incorporated a EO-5012M 5 MP monochromatic CMOS camera. The camera was mounted on a VZM 1000i 2.5X-10X variable zoom imaging lens. When incorporated with the $1 / 2.5$ " sensor within the camera, camera, the field of view ranged from $2.24 \times 1.68 \mathrm{~mm}$ to $0.56 \times 0.42 \mathrm{~mm}$ for the minimum and maximum zoom respectively. The target was silhouetted using a $470 \mathrm{~nm}$ Blue Metaphase technology collimated spotlight. This setup, shown in Figure 4.13: 


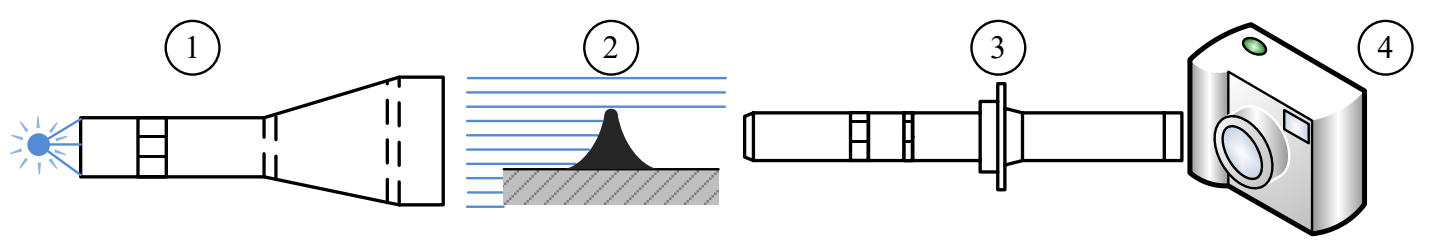

Figure 4.13: Diagram of imaging setup. (1) Blue LED collimated backlight source, (2) Ferrofluid droplet on slide within Helmholtz coil (not shown), (3) Variable zoom imaging lens, (4) Monochromatic CMOS camera.

A Helmholtz coil, diagramed in Figure 4.14, generated a variable uniform magnetic field. The coil consisted of a pair of solenoids, each containing 100 windings. The coil and power supply can generate a maximum field of 310 gauss. Slots in the coil core allowed for light to pass through to backlight the droplet and provide imaging access. Coil current was measured with a Hall effect current sensor and a calibration relationship was obtained with the use of an Alpha Labs GM-2 Gauss Meter with a High Stability Universal Probe. The fluid droplet was biased by applying a voltage to the electrode (2) in Figure 4.14, using an UltraVolt High Voltage Amplifier (HVA) module with a range of $\pm 5 \mathrm{kV}$. The biased and grounded electrodes were separated by $4 \mathrm{~mm}$. Images of the experimental setup are shown in Figures 4.15 and 4.16.

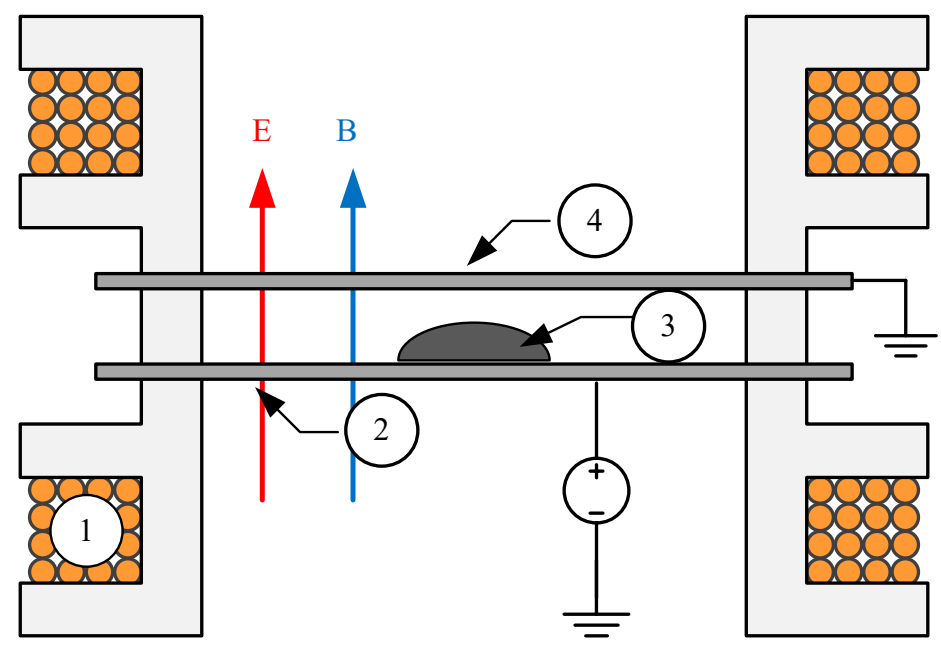

Figure 4.14: Cross section of Helmholtz coil apparatus. The coil inner diameter is 3.8 cm. (1) Coil with 100 wraps per solenoid, (2) Biased electrode supporting droplet, (3) Ferrofluid droplet, (4) Grounded electrode. Spacing between the two electrodes is 4 mm. 


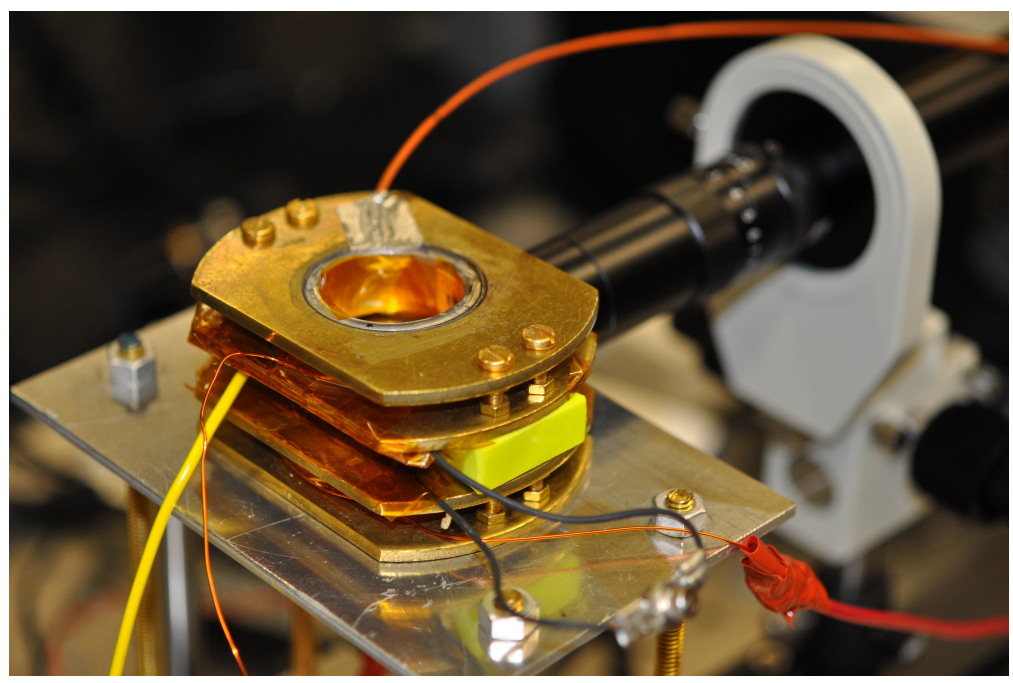

Figure 4.15: Image of Helmholtz apparatus.

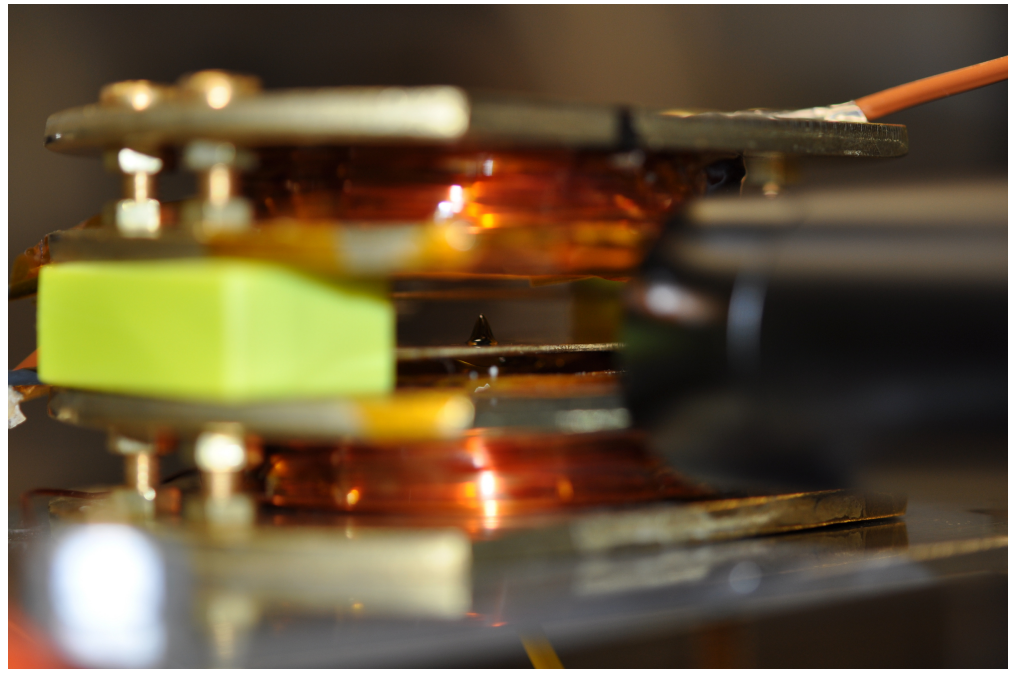

Figure 4.16: Ferrofluid droplet within Helmholtz coil.

Brass was selected for the electrode material for the desirable wetting characteristics it demonstrated with the ionic liquid ferrofluid. The droplet volumes and contact energy were sufficient to constraint the droplet to a regime where only a single peak would develop. The fluid was placed on the electrode in the presence of a magnetic field. Images were then taken incrementally, increasing the electric field for each subsequent image. A sample image obtained from this system is shown below in

Figure 4.17. Silhouette images were then processed to perform edge detection and volume integration. 


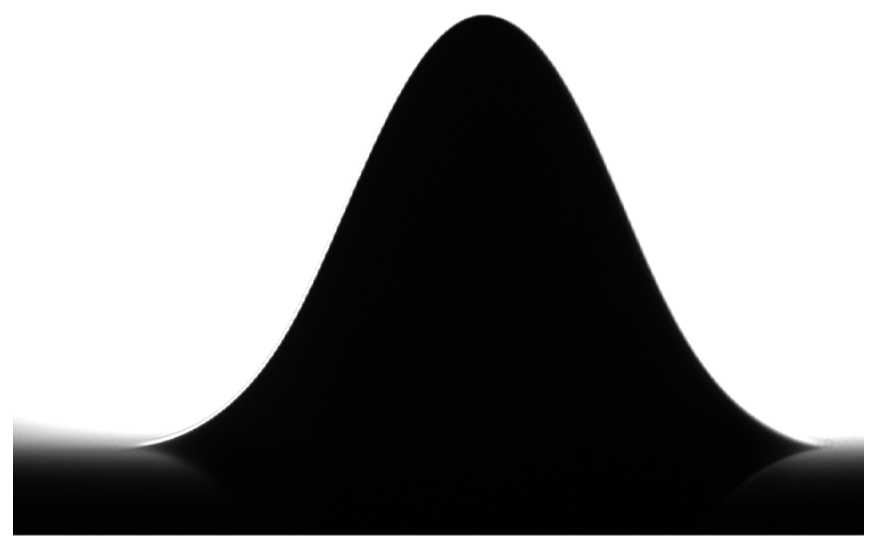

Figure 4.17: Sample image acquired of ferrofluid peak within Helmholtz coil. Peak volume was $0.2688 \mathrm{~mm}^{3}$ and the applied magnetic field was 203 gauss.

Previous work by Gollwitzer has suggested the possibility of a magnetic-fielddependent hysteresis in the height of a ferrofluid peak, which was attributed the fluid wetting the container. ${ }^{84}$ For the ferrofluid used in this study, hysteresis was observed prior to the droplet becoming pinned, as shown in Figure 4.18. It was found that hysteresis could be minimized by reducing the magnetic field to zero following insertion of the droplet. When the field was reapplied, the droplet would be pinned and would still form only a single peak; this pinned peak had negligible hysteresis during subsequent changes in magnetic field strength.

Operating at peak current, the Helmholtz coil dissipates approximately 150 watts of power. Surface tension and magnetization are both a strong function of temperature. To minimize temperature influences, the slide holding the sessile droplet was thermally insulated from heat sources using Teflon and PLA. To quantify temperature effects, a droplet of ferrofluid was imaged under constant fields for 120 seconds and the change in height was measured. During the time required to obtain an image set ( $\sim 60$ seconds), the height variation was observed to be less than 2 percent of the initial apex height as can be observed in Figure 4.19. This change can be attributed to temperature variations and the low creep velocity of the contact point. In all subsequent testing data collection was 
completed within 60 seconds of applying the magnetic field to render the temperature effect negligible.

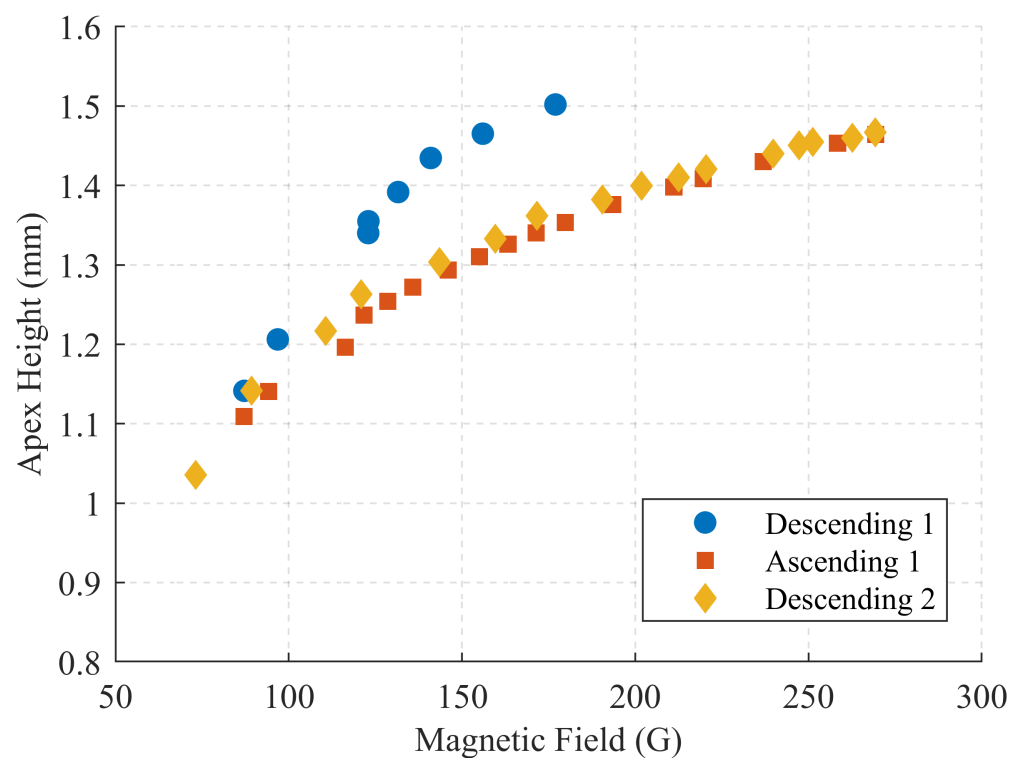

Figure 4.18: Tip height for a fluid droplet with a volume of $1.4135 \mathrm{~mm}^{3}$. The droplet was placed in a strong magnetic field using a syringe. The field strength was then decreased and increased again as a series of images were taken.

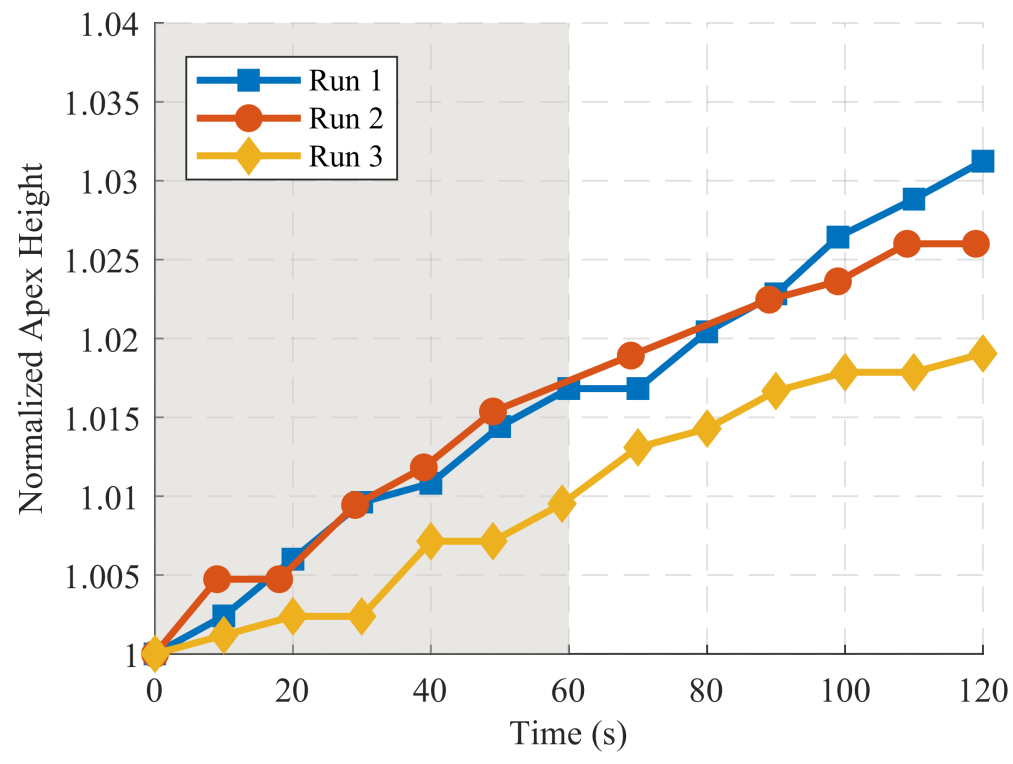

Figure 4.19: Study of droplet height sensitivity to magnet coil heating. Tip height was normalized against the initial height such that droplets of different volume could be plotted together. If the droplet is imaged within 60 seconds of applying the Helmholtz field, then the temperature-induced height change is less than $2 \%$. 


\subsubsection{Simulation Results and Comparison with Experiment}

Four sets of laboratory images will initially be discussed in this section. Elements of these sets are presented below in Figure 4.20. The droplet shown was exposed to four different magnetic field strengths; for each magnetic field the voltage between the electrodes was increased from 0 to 4000 volts in 100-volt increments.

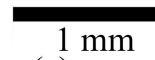

(a)
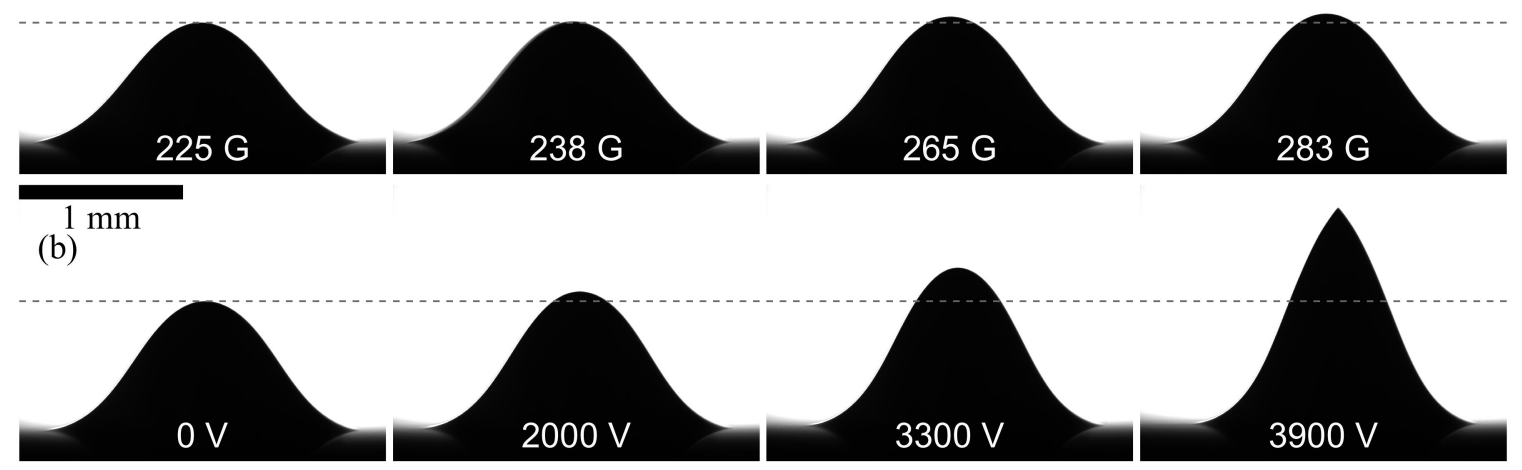

Figure 4.20: (a) Droplet meniscus under magnetic field only. (b) Droplet meniscus under magnetic and electric field held under a constant magnetic field of 282 gauss. Droplet volume was $0.616 \mathrm{~mm}^{3}$. Electrodes were spaced $4 \mathrm{~mm}$ apart.
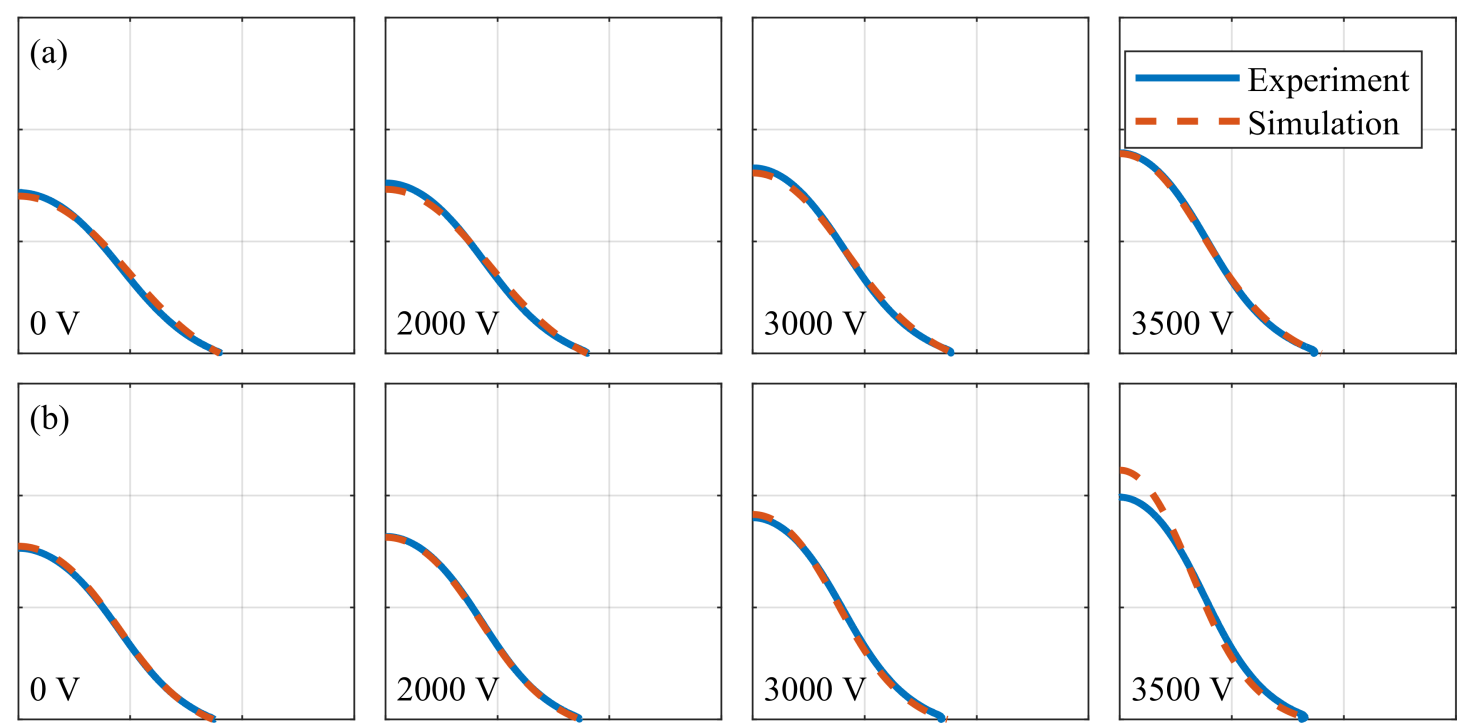

Figure 4.21: Select simulated and imaged contours. (a) 225 gauss and (b) 264 gauss. 
Dynamic simulations were performed for each of the four sets of images. The simulated profiles and silhouette images from laboratory data were found to have excellent agreement for voltages up until approximately $85 \%$ of the onset voltage, after which the simulations slightly over-predict the meniscus deformation. Full meniscus profile comparisons are shown above in Figure 4.21.

The apex height is presented in terms of the applied voltage in Figure 4.22 and in terms of Bond numbers in Figure 4.23.The electric Bond number, $B_{e}$, was calculated using the apex electric field, $E_{a}$, which is the field that exists at the tip of the meniscus and is thus strongly dependent upon the meniscus geometry. For the simulated results retrieval of $E_{a}$ is trivial. For the experiment $E_{a}$ was extracted by importing the measured meniscus silhouette geometry into an electrostatic solver as a rigid (non-deformable) conductor and using the electrode geometry and applied potential to calculate the field at the apex. The magnetic Bond number, $B_{m}$, was calculated using $H_{0}$, which is the vacuum magnetizing field created by the Helmholtz coil without the presence of ferrofluid. The scaling dimension $R_{0}$ was set to be the radius of an equivalent-volume hemisphere.

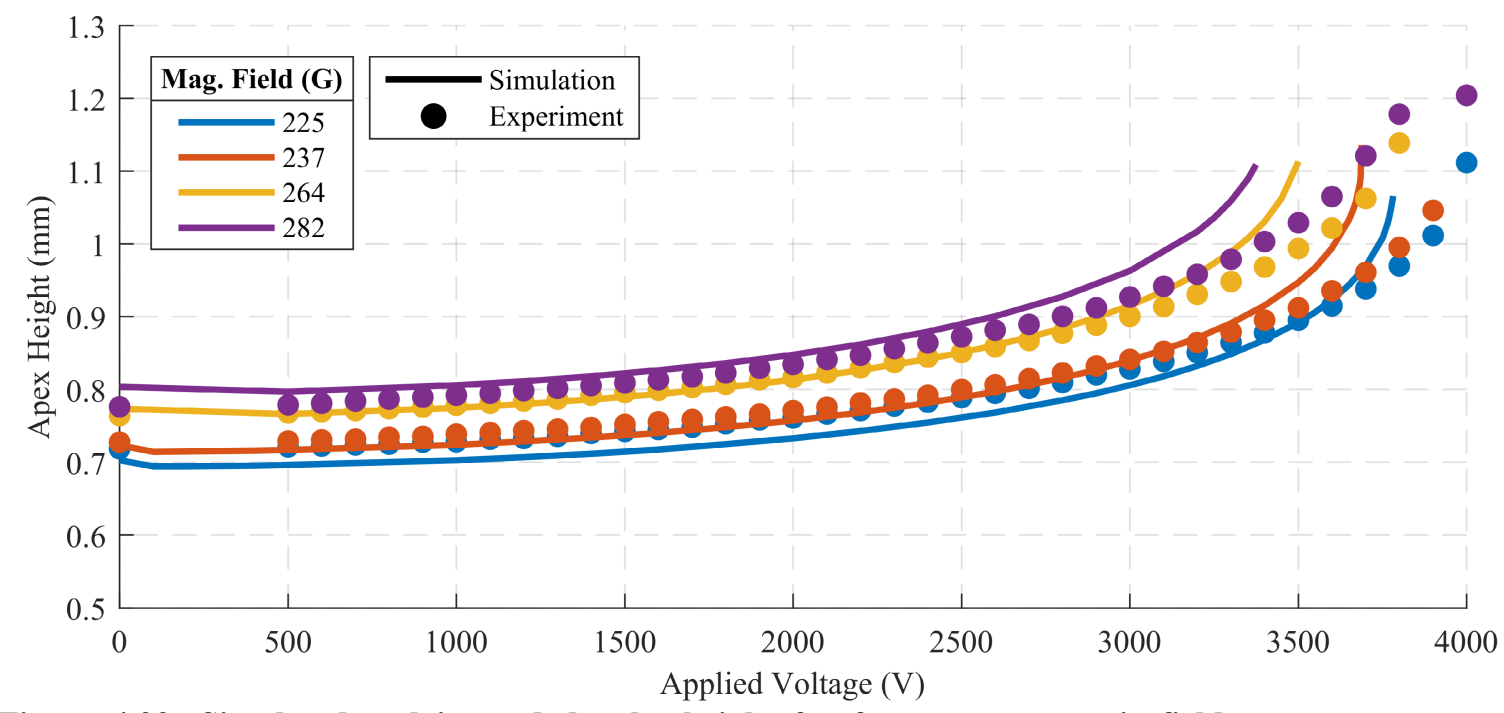

Figure 4.22: Simulated and imaged droplet height for four mag magnetic fields. Droplet volume of $0.616 \mathrm{~mm} 3$ 


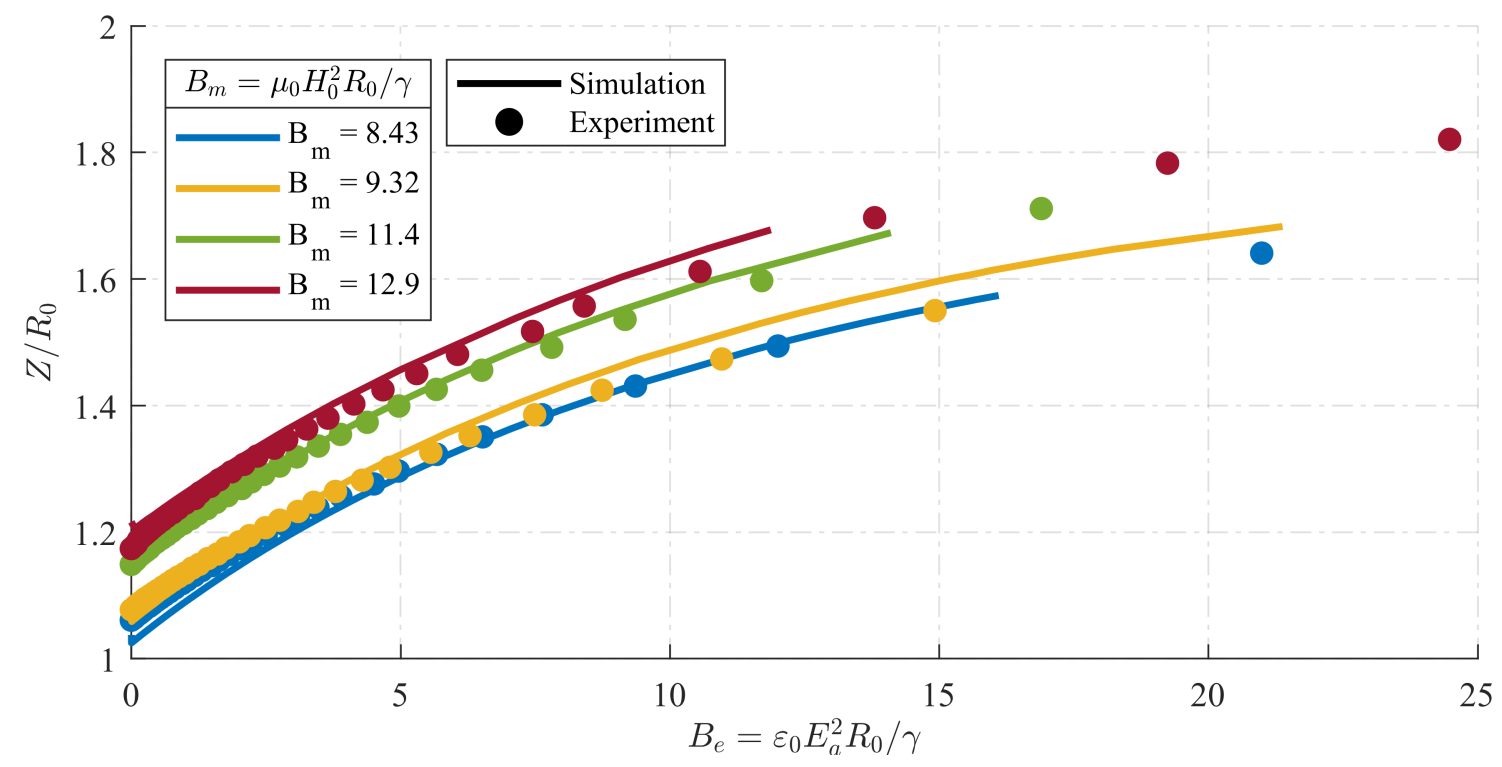

Figure 4.23: Simulated and imaged droplet height scaled by $R_{0}$ plotted against the electric Bond number for four magnetic fields. Droplet volume of $0.616 \mathbf{~ m m}^{3}$.

The electric and combined magnetic stress components, $P_{e}=(1 / 2) \varepsilon_{o} E_{n}^{2}$ and $P_{m}=(1 / 2) \mu_{0} M_{n}^{2}+\mu_{0} \int_{0}^{H} M d H$, at the meniscus apex for a single simulated droplet under constant magnetic field are presented in Figure 4.24. It can be observed that the combined magnetic stresses dominate at low applied voltages but are rapidly overtaken by the electric stress near onset of spray. As the magnitude of the applied voltage increases the electric stress at the meniscus apex grows until it is the dominant perturbation to the interface. This is in part because the apex surface grows closer to the counter electrode, but more strongly because the apex radius of curvature decreases. The magnetic stresses remain relatively constant, compared to the electric stresses, but increase slightly as the applied voltage increases. 


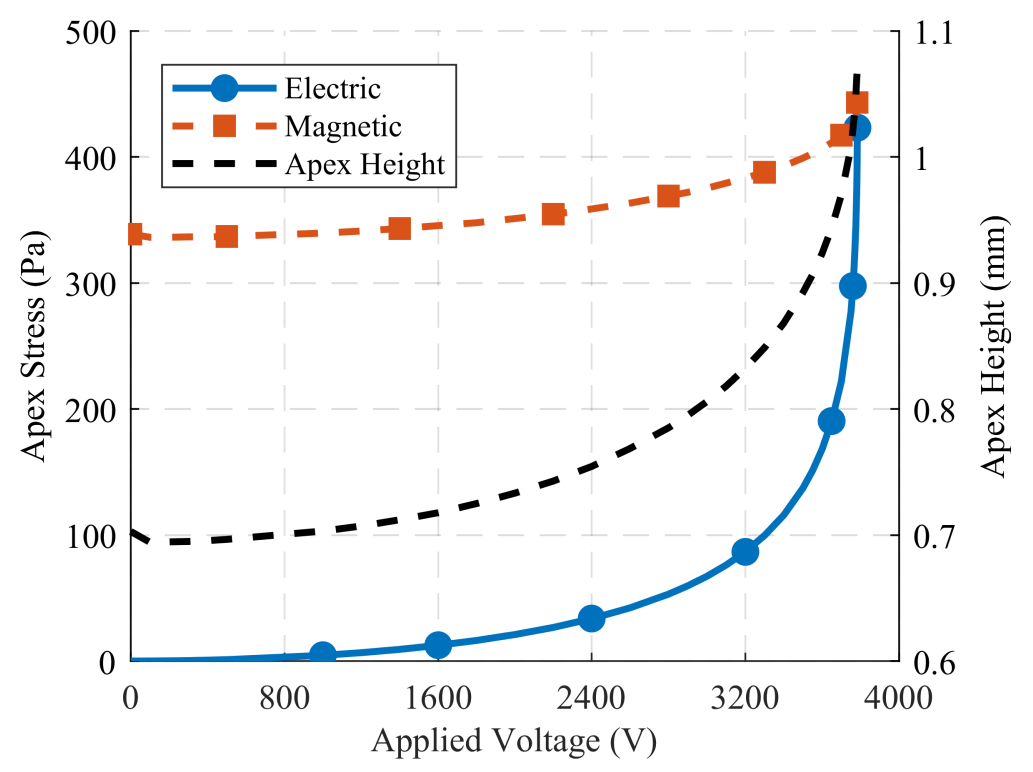

Figure 4.24: Apex components of electric and magnetic stresses. The electric component of the interface stress becomes asymptotic indicating run-away to onset. Simulation of a $0.616 \mathrm{~mm}^{3}$ droplet in a $225-\mathrm{G}$ magnetic field.

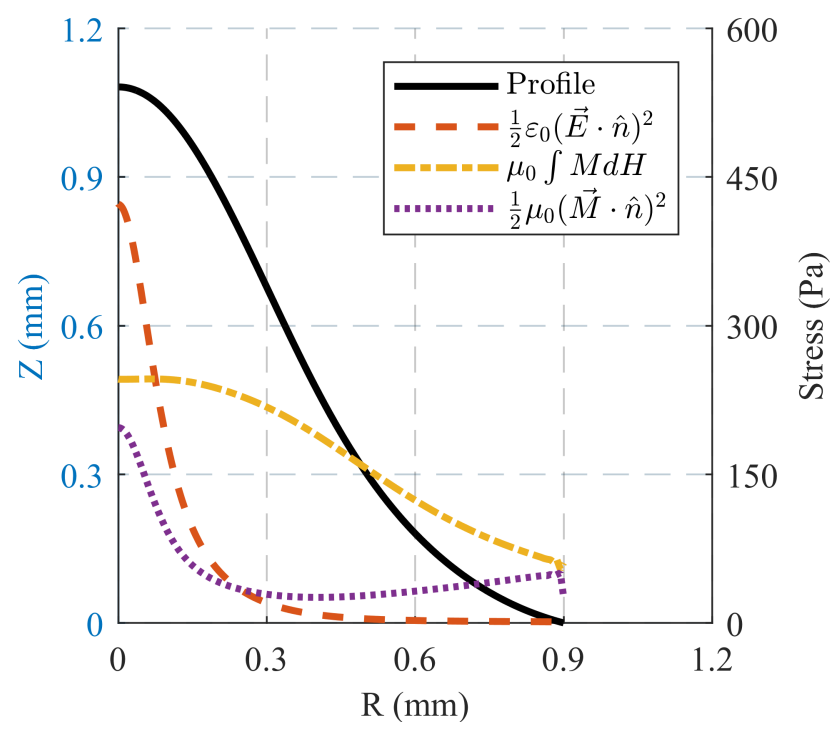

Figure 4.25: Electric and magnetic stress components along fluid interface for $\mathrm{V}=$ $3783 \mathrm{~V}$. Simulation of a $0.616 \mathrm{~mm}^{3}$ droplet in a $225-\mathrm{G}$ magnetic field.

The individual stress components along the entire fluid interface are presented in Figure 4.25 for a single applied voltage. The fluid magnetic-pressure, $\mu_{0} \int M d H$ term, has a significant role along the entire interface. Meanwhile, the magnetic-normal term, 
$(1 / 2) \mu_{0} M_{n}^{2}$, closely resembles the analogous electric-normal stress, $(1 / 2) \varepsilon_{0} E_{n}^{2}$, with both terms increasing rapidly and reaching their maximum at the fluid apex.

\subsection{Conclusions}

The computational fluid dynamics modeling technique developed and presented in this chapter simulated the fluid interface of both a capillary needle and a normal-field instability electrospray source under both electric and magnetic stresses prior to onset of emission. A metric was established to indicate run-away to onset of emission for a capillary needle, which agreed well with onset observed experimentally. For the case of the normal-field instability, silhouette images were taken of sessile droplets exposed to combined magnetic and electric fields. Modeling droplets under matching field conditions yielded excellent agreement with the imaged profiles.

Analysis of the stress components along the fluid interface reveal that the magnetic stress is instrumental in preconditioning the fluid for the electric stresses. When onset of emission is approached, the electric stress rise exponentially in the apex region until they dwarf the magnetic stresses. This coupled behavior reduces the voltage necessary to achieve onset of emission. 



\section{Chapter 5 Emission Studies of an ILFF Electrospray via the Normal-Field Instability}

Work by E.J. Meyer, ${ }^{30,} 91$ and Mkrtchyan et al. ${ }^{29}$ demonstrated that electrospray emission can be achieved via a combined magneto-electric instability without the need of supporting structures. However, this work was limited to short-duration emission. To serve as a viable solution for nanosatellite thrusters, ionic liquid ferrofluid electrospray sources emitting via the normal-field instability must be capable of operating stably for extended periods of time. In this chapter, work performed by E.J. Meyer will be expanded upon with the goal of understanding of the operational behavior of a ferrofluid electrospray source emitting from a normal-field instability.

The work presented in this chapter describes the emission behavior of a single peak ferrofluid electrospray source. A single peak was obtained by sizing a fluid reservoir such that the boundaries would permit only a one instability to form. The fluid reservoir was not able to be replenished as the emission depleted the fluid level, thus limiting emission to around 16 hours.

The single peak emitter will be referred to as a normal-field source hereafter in this

dissertation. However, as defined by Rosensweig, ${ }^{55}$ the normal-field instability, discussed in Section 3.3, is a pattern of spikes which results when a critical magnetic field is applied to a pool of ferrofluid. When the emission reservoir for a single tip emitter, which is presented in Section 5.2.1, is infused in the absence of a magnetic file, a critical magnetization is less apparent when a field is later applied. Instead, a gradual transition from a flat surface to a dome is observed, which rapidly transforms into a peak at higher field strengths. This observation suggests that edge effects are not inconsequential and can 
provide an initial curvature to the fluid surface which weakens the normal-field instability mechanism in this configuration.

\subsection{Goals of Study}

The primary goals of this study were to (1) demonstrate long-duration stable emission of the source, and estimate propulsion performance parameters, (2) determine the sensitivity with respect to changes in the extraction potential, and (3) identify factors impacting the operational behavior of the source. Section 5.2 describes the equipment, electrospray source, and testing methodology. Section 5.3 discusses the influence of peak sharpness on emission behavior. Finally, Sections 5.4 and 5.5 present the long duration emission results and the sensitivity of the emission source in response to changes in extraction potential.

\subsection{Equipment and Testing Methodology}

The design and operation methodology of the electrospray source play a considerable factor in how the source will ultimately behave while emitting. The purpose of this section is to describe electrospray source, current collection technique, vacuum facility in which the testing was performed and finally the testing methodology.

\subsubsection{Electrospray Source}

The electrospray emitter apparatus utilized for this study was designed by Terhune ${ }^{35}$ with modifications to the manner in which the extractor and collector electrodes were mounted. A diagram of the emitter apparatus is presented in Figure 5.1 and an image of the apparatus with a filled reservoir is shown in Figure 5.2. 


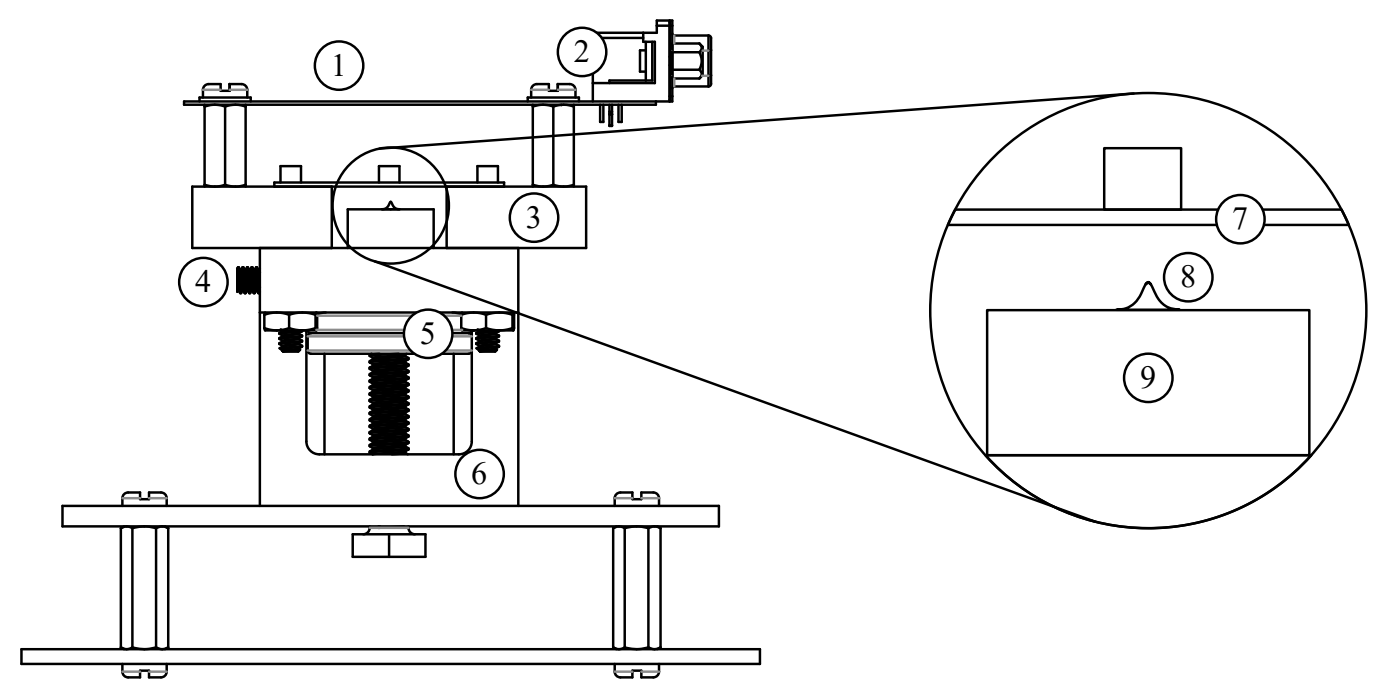

Figure 5.1: Electrospray emission apparatus. (1) current collector, (2) DB-9 surface mount connector, (3) extractor alignment mount, (4) electrical bias terminal, (5), N52 magnets (1-inch diameter), (6) Delrin isolation block, (7) Extractor electrode, (8) ferrofluid instability, and (9) fluid reservoir.

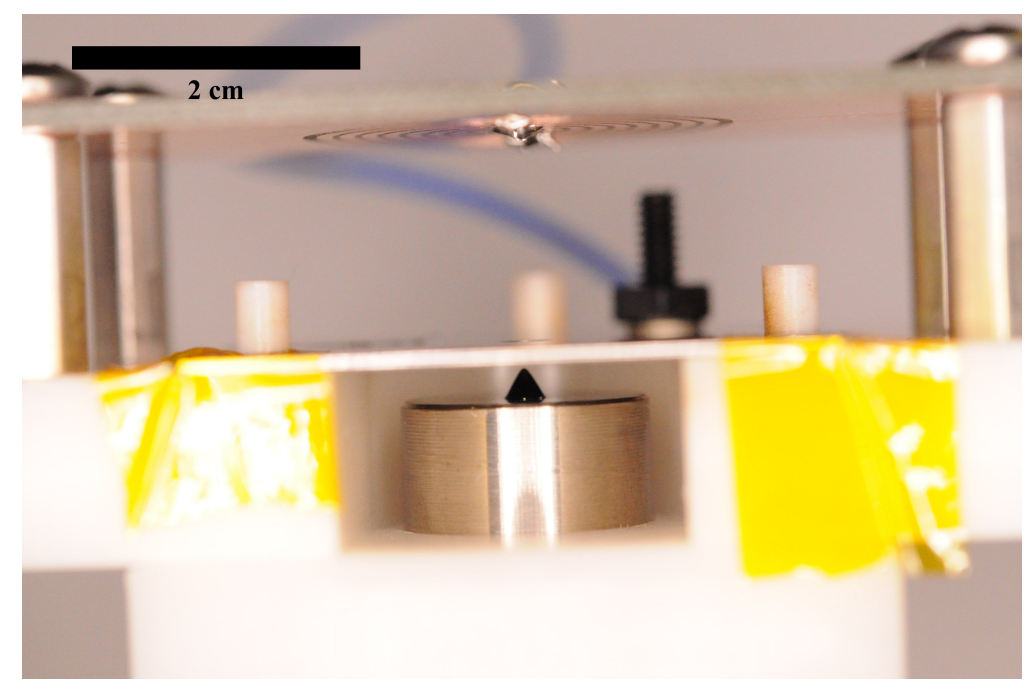

Figure 5.2: Frontal view of the assembled ILFF electrospray emission apparatus.

The extractor electrode was positioned $2.85 \mathrm{~mm}$ downstream of the fluid reservoir. The current collector and the extractor were separated by $15.50 \mathrm{~mm}$ with an extractor thickness of $1.13 \mathrm{~mm}$.

A dimensioned schematic of the fluid reservoir is presented in Figure 5.3. The reservoir was infused with $14 \mu \mathrm{L}$ of ferrofluid, in the presence of a magnetic field, using a $50-\mu \mathrm{L}$ syringe and syringe pump. Fluid was infused into the reservoir at a flow rate of $1 \mu \mathrm{L} / \mathrm{s}$. A 
linear translation stage was utilized to control the vertical spacing of the syringe relative to the fluid reservoir.
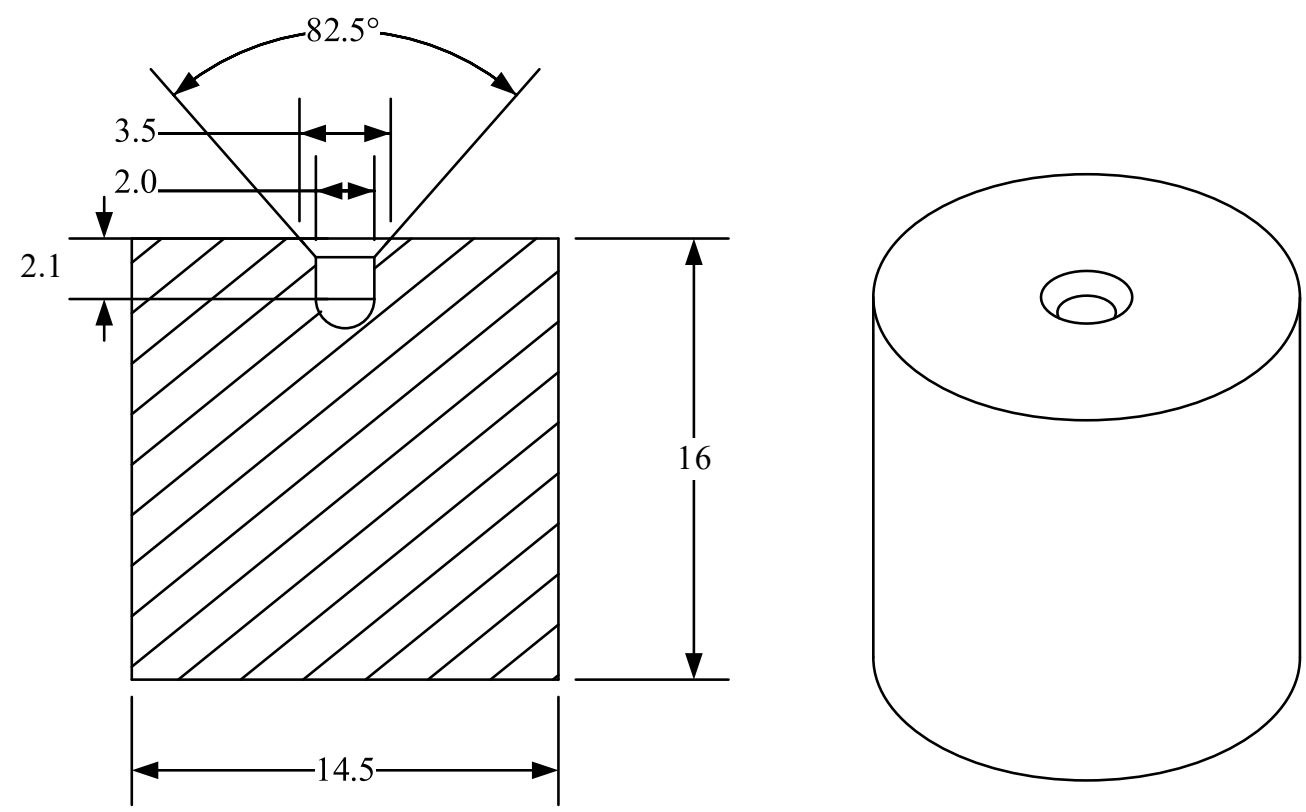

Figure 5.3: Sectional and isometric view of fluid reservoir. All dimensions in millimeters.

The well volume of the fluid reservoir has a volume of $10.7 \mu \mathrm{L}$. A small amount of fluid was lost in the transfer process, predominately a result of wetting the slightly magnetic syringe needle. Due to the uncertainty in the amount of fluid delivered, actual volume of the peak was estimated by performing a volume integral on an image of the peak using the extractor diameter as a reference dimension. This process will be discussed in more detail in Section 5.2.5. The fluid was then allowed to sit at vacuum ( $<1$ torr) for at least 2-hours prior to being sprayed.

\subsubsection{High Voltage Power Supply}

The high voltage necessary to achieve electrospray emission was provided by a UltraVolt HVA 5HVA precision high voltage amplifier. The amplifier, proving $500 \mathrm{~V} / \mathrm{V}$ amplification, was commanded externally with a Rigol DG4162 waveform generator. The commanding signal was a $500 \mathrm{mHz}$ ( 2 second period) square wave centered at $0 \mathrm{~V}-$ yielding equal magnitude extraction voltage in both polarities. The biasing configuration 
of the source resulted in positive emission current in response to a positive biasing potential.

Lozano and Martínez-Sánchez observed alternating extraction eliminates electrochemical effects which are observed with continuous DC emission. ${ }^{93}$ When switching to AC extraction, the bubbling phenomenon observed by Meyer ${ }^{30}$ at the base of the normal-field emitter was not observed. Performance parameters of the high voltage amplifier are presented in Table 5-1.

Power was transferred from the supply to the vacuum facility using a RG8 coaxial cable. During bipolar operation, large currents were observed following the switch in polarity, which quickly decayed. Several examples of this waveform are shown in Figure 5.4. This phenomenon was attributed to the capacitance and inductance of the coaxial cable. A similar feature was not observed on the low voltage side where the emission current was measured for the duration of testing. The primary purpose of the high voltage current measurement was to obtain a current balance to detect secondary electron. It was decided to simply perform the current balances after the transient had died out than to subtract the transient. This approach was selected since the case-to-case variations in the transient were comparable to the variation in the emission current.

Table 5-1: UltraVolt HVA 5HVA Performance Values ${ }^{94}$

\begin{tabular}{l|l|l}
\hline Parameter & & Value \\
\hline \hline Max Current & $(\mu \mathrm{A})$ & 200 \\
\hline Voltage Monitor & $(\mathrm{V})$ & 0 to $\pm 10, \pm 0.5 \%$ \\
\hline Current Monitor & $(\mathrm{V})$ & 0 to $\pm 10, \pm 1 \%$ \\
\hline Slew Rate & $(\mathrm{V} / \mu \mathrm{s})$ & 40 \\
\hline Bandwidth at $8 \mathrm{kV}$ & $(\mathrm{Hz})$ & $2500($ No load $)$ \\
& & $500(100 \mathrm{pF}$ load $)$ \\
\hline
\end{tabular}




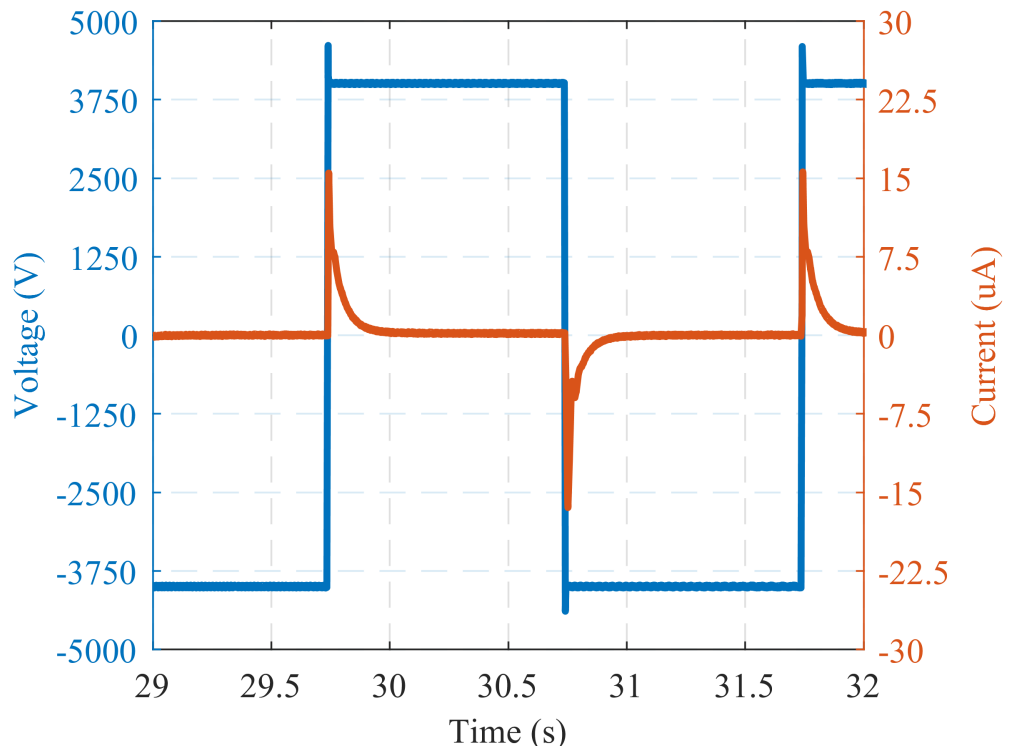

Figure 5.4: Spike in measured current observed on the high voltage side of the electrospray apparatus during AC excitation. Phenomenon is observed with and without fluid in the reservoir and is attributed to the charging of the coaxial power cable. Waveform collected with a sample frequency of $2000 \mathrm{~Hz}$.

\subsubsection{Segmented Faraday Probe and $\mu \mathrm{A}$ Current Measurement}

A segmented current collector was utilized for this study to measure the emission current of the electrospray source. A segmented collector provided two advantages: (1) the concentric circular collectors enabled angular divergence measurements of the plume to be obtained and (2) a lower collection area enabled a high percentage of the full-scale range of the current transducers to be utilized.

The segmented Faraday probe was milled out of two-sided copper clad board. Each of the nine collector pads was routed to a pin on a board-mounted DB-9 receptacle. A PCB layout of the board is shown in Figure 5.5 and a milled board is presented in Figure 5.6. Prior to testing, each board was submerged in a 3\% acetic acid solution for 30 seconds to remove any tarnish. The boards were then immediately submerged in a $95 \%$ ethyl alcohol/water solution and placed in an ultrasonic bath for 30 minutes. The collectors were then dried with a lint free cloth and placed in a vacuum to dry completely. A cleaned board was used for each emission test. 


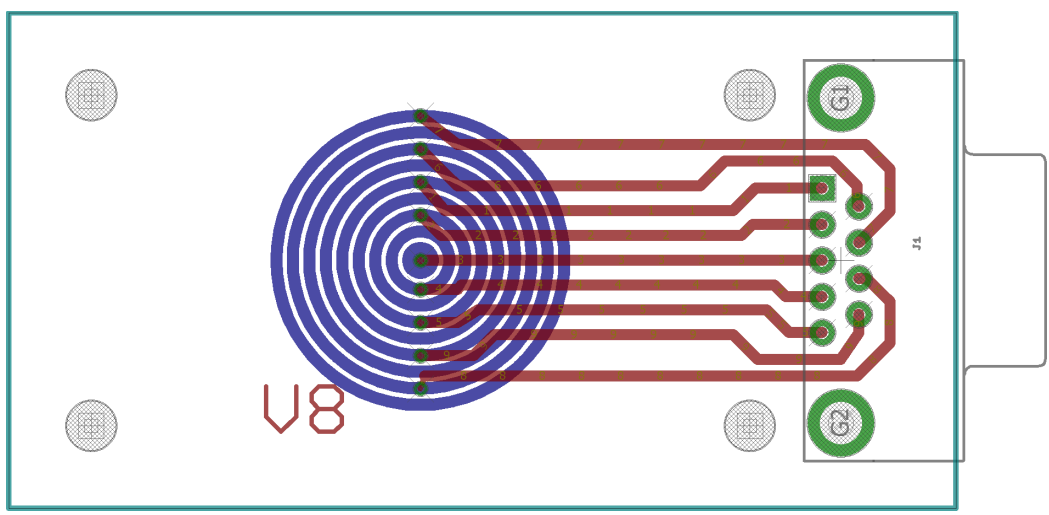

Figure 5.5: Circuit board schematic for copper clad current collector (width=2.875 in, height $=1.5 \mathrm{in})$.

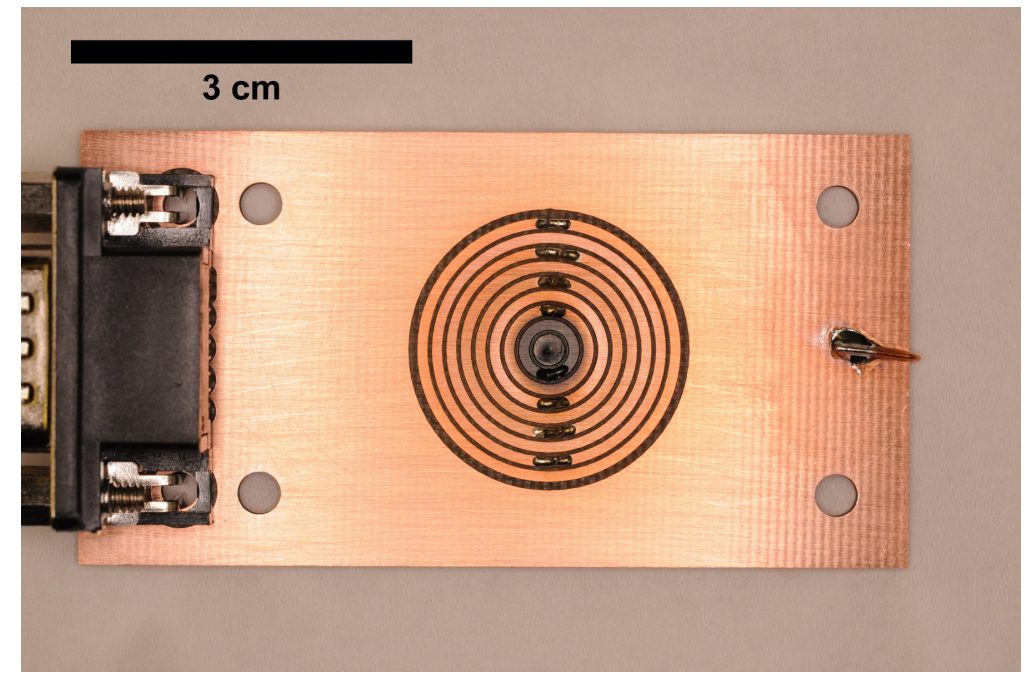

Figure 5.6: Current collector after approximately 15 hours of emission. Ferrofluid buildup can be observed on the center rings with decreasing intensity as the radial distance increases. Continuity between adjacent pads was measured after testing to detect pad-to-pad shorts. None were ever detected.

The collected current on each of the nines segments of the probe was measured using an EEVblog $\mu$ Current ${ }^{\mathrm{TM}}$ (original) precision current meters. These meters were operated on the $1 \mathrm{mV} / \mathrm{nA}$ scale, providing a full-scale range of $\pm 1500 \mathrm{nA}$ with a $2 \mathrm{kHz}$ bandwidth. ${ }^{95}$ Buildup of ferrofluid particles was observed on the collector during the testing. Upon completion of the test, the probe was checked for pad-to-pad continuity by biasing the center collector up to $500 \mathrm{~V}$ and measuring the adjacent collector. The collector-to-collector resistance was typically on the order of $250 \mathrm{M} \Omega \mathrm{s}$, indicating that the conductive film coating the surface provided a conductive, albeit small, path between adjacent collectors. Relative to the collector-to-ground resistance, the collector-to-collector path is negligible. 
Close agreement was observed between emission-side current and intercepted current. Collected current averaged $85 \%$ of emission current for both positive and negative polarities. Consequently, secondary electron emission is not believed to be a significant factor. A typical balance between emitted and intercepted current is presented in Figure 5.7. Intercepted current on the extraction electrode was not measured. However, buildup was not observed on the face or within the opening of the extraction electrode facing the fluid reservoir, which would have required an emission half-angle $>45$ degrees, much greater than the emission angles observed which will be presented in Section 6.3.

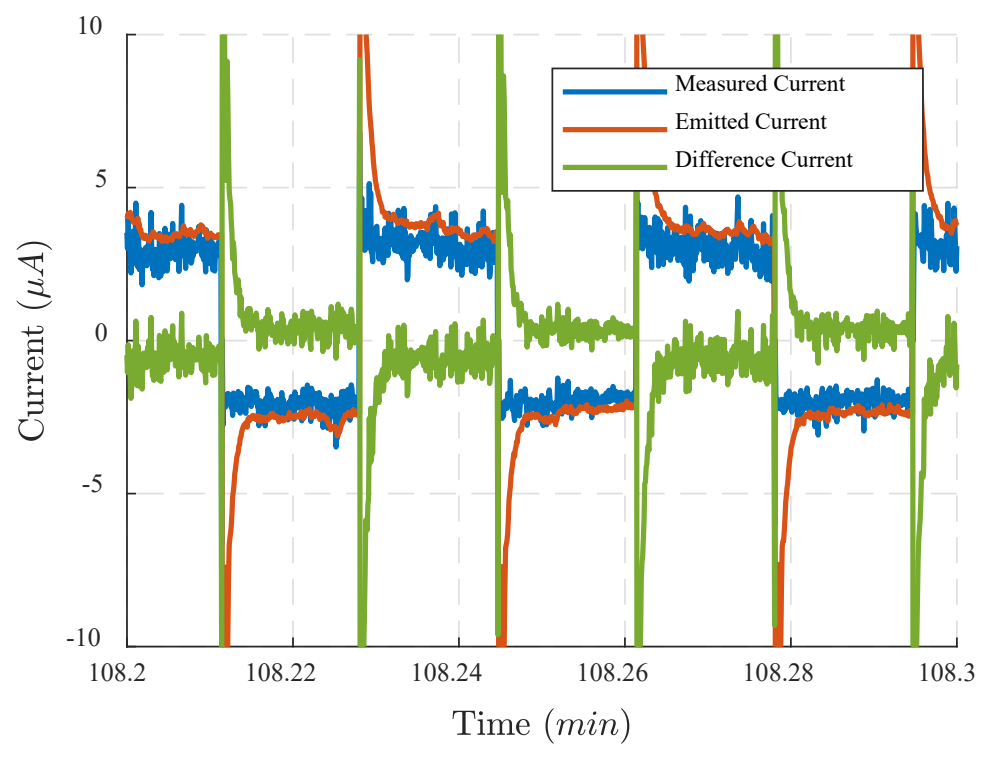

Figure 5.7: Typical balance between emitted and collected current. Transient spikes are present in the supply current resulting from the charging and discharging of the coaxial cable between the supply and vacuum facility.

Deposited material was observed on the side of the extraction electrode facing the segmented collector. A typical level of accumulation is shown in Figure 5.8. This material was easily removed using acetone and wiping with a Kimwipe - restoring the electrode to a mirror polish similar to that prior to emission. Since no permanent damage was done to the electrode surface, it is unlikely the discoloration is a result of surface damage. It is likely that the observed material is emitted fluid which did not stick to the collector and instead ricocheted towards the extractor. Measuring the current intercepted likely would have confirmed this hypothesis - accounting for a portion of the $15 \%$ mismatch between emitted and collected current. 


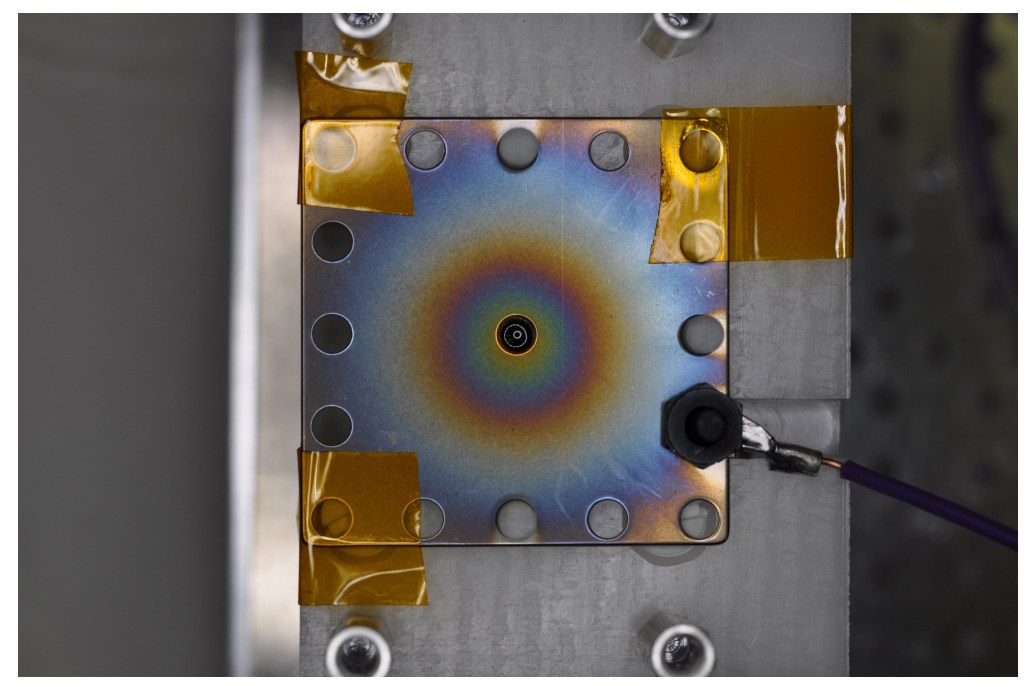

Figure 5.8: Material build up on face of extraction electrode facing the collection electrode.

\subsubsection{Vacuum Facility}

Testing was performed using the Micropropulsion Facility-2, shown in Figure 5.9, within the Ion Space Propulsion Laboratory at Michigan Technological University. This facility is approximately a 60-liter cylinder and is roughed down using a 300 liter $/ \mathrm{min}$ triscroll pump and a 280 liter per second turbomolecular pump. The base pressure of this facility is approximately $4 \times 10^{-7}$ torr. Testing was conducted when the tank pressure reached approximately $1 \times 10^{-6}$ torr. A single emitter was found to have no measurable impact on tank pressure and tank pressure was observed to drop continuously during testing until the base pressure was obtained. 


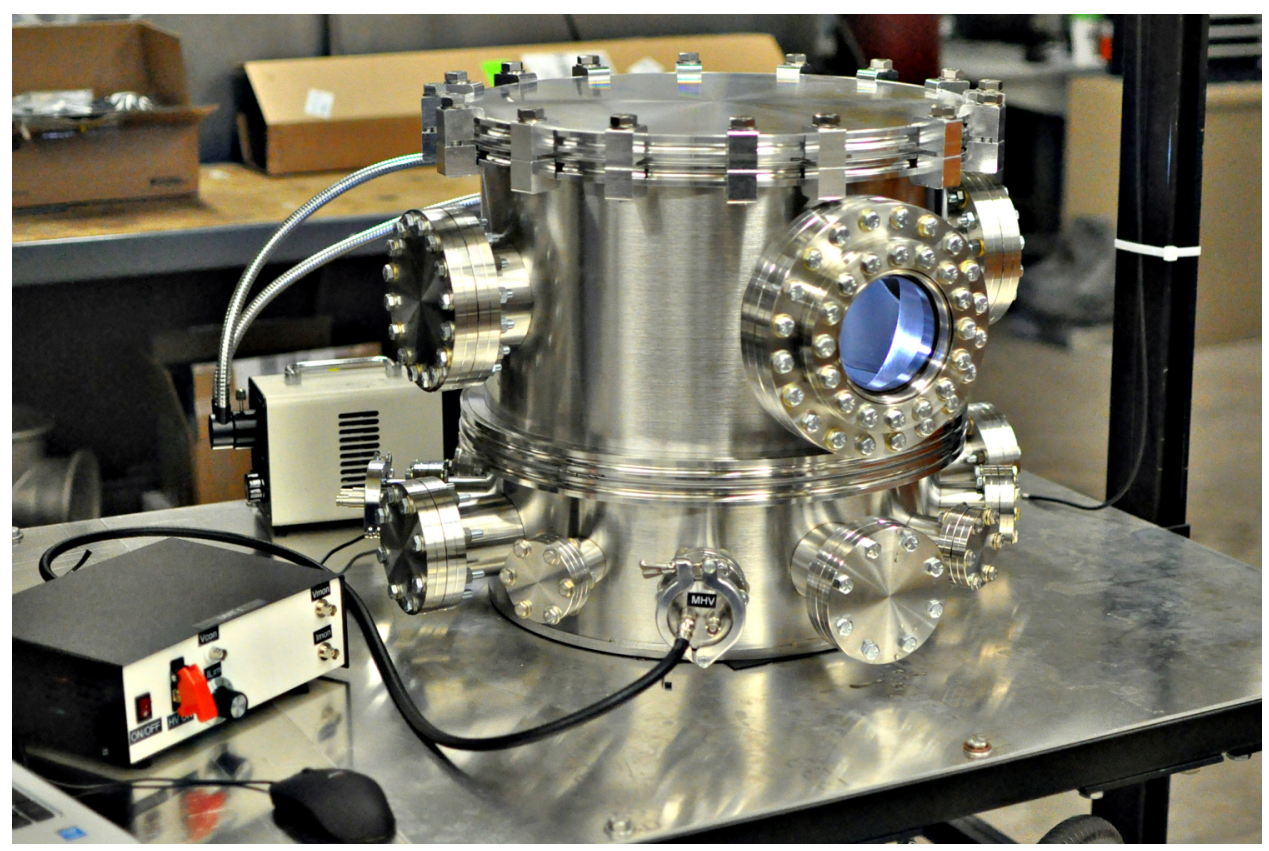

Figure 5.9: Micropropulsion vacuum facility in which emission testing was performed. A stereo microscope (not shown) enabled high magnification viewing of the emission source during testing.

\subsubsection{Testing Methodology and Telemetry Phases}

The fluid was biased with a $500 \mathrm{mHz}$ bipolar square wave with equal positive and negative polarities. While emitting, the fluid was imaged using a camera integrated with microscope. Time-lapse images were taken of the tip using a 5.1 MP camera at intervals of 30 -seconds. Post processing of these images permitted the tip volume to be measured by revolving and integrating the fluid interface. By differentiating the tip volume with respect to time, the volumetric and mass flow rate of the source were estimated. A detailed description of the methodology employed to estimate mass flow rate is presented in detail in Appendix C.

Three distinct phases of the emission telemetry materialized. These were (1) startup, (2) constant voltage mode, and (3) shutdown or terminal phase. During the start-up phase electrospray source, the fluid peak was very sensitive and required frequent management of the extraction potential to maintain emission. After a span of 20 to 180 minutes, the emission would stabilize and transform into a constant voltage mode where emission would 
be sustained at a relatively constant emission current for spans in excess of 10 hours without requiring further increases in extraction potential. At the conclusion of the constant voltage emission mode, the source would self-extinguish, and the terminal phase would start. During this phase, the extraction potential would need to be regularly increased to restart the source. The source would then spray for a period of $10 \mathrm{~s}$ of seconds to minutes before spray would cease again. The extraction potential was then increased again in a cyclical process until fluid would be depleted or the maximum range of the voltage source was reached. The startup and terminal phase of the electrospray telemetry are highlighted in Figures 5.10 and 5.11, respectively.

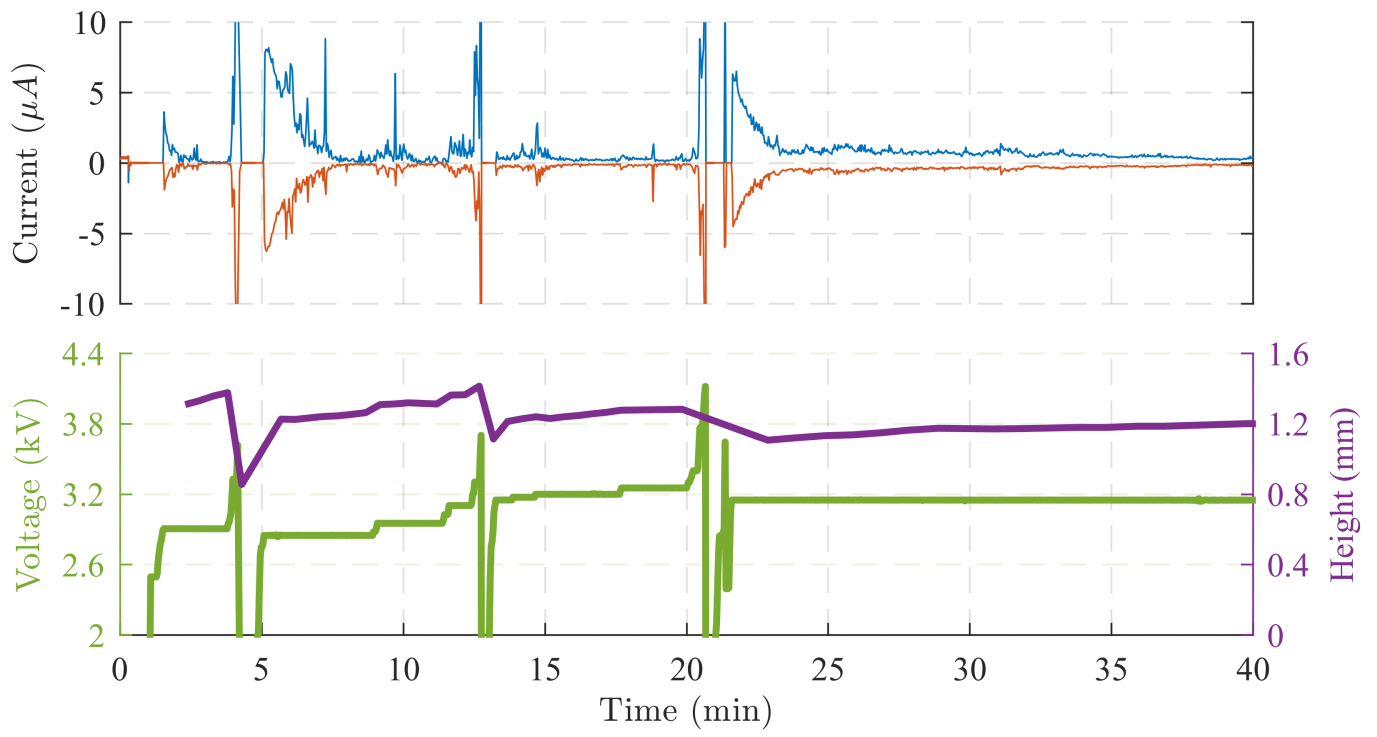

Figure 5.10: Sample startup of electrospray source telemetry. The extraction potential is increased until onset of emission is achieved. The extraction potential is then periodically increased to maintain emission. If a temporary spike in emission current exceeds the range of the current transducers, the extraction voltage is reduced to zero and slowly increased again. The source will eventually transition into a constant voltage emission mode. 

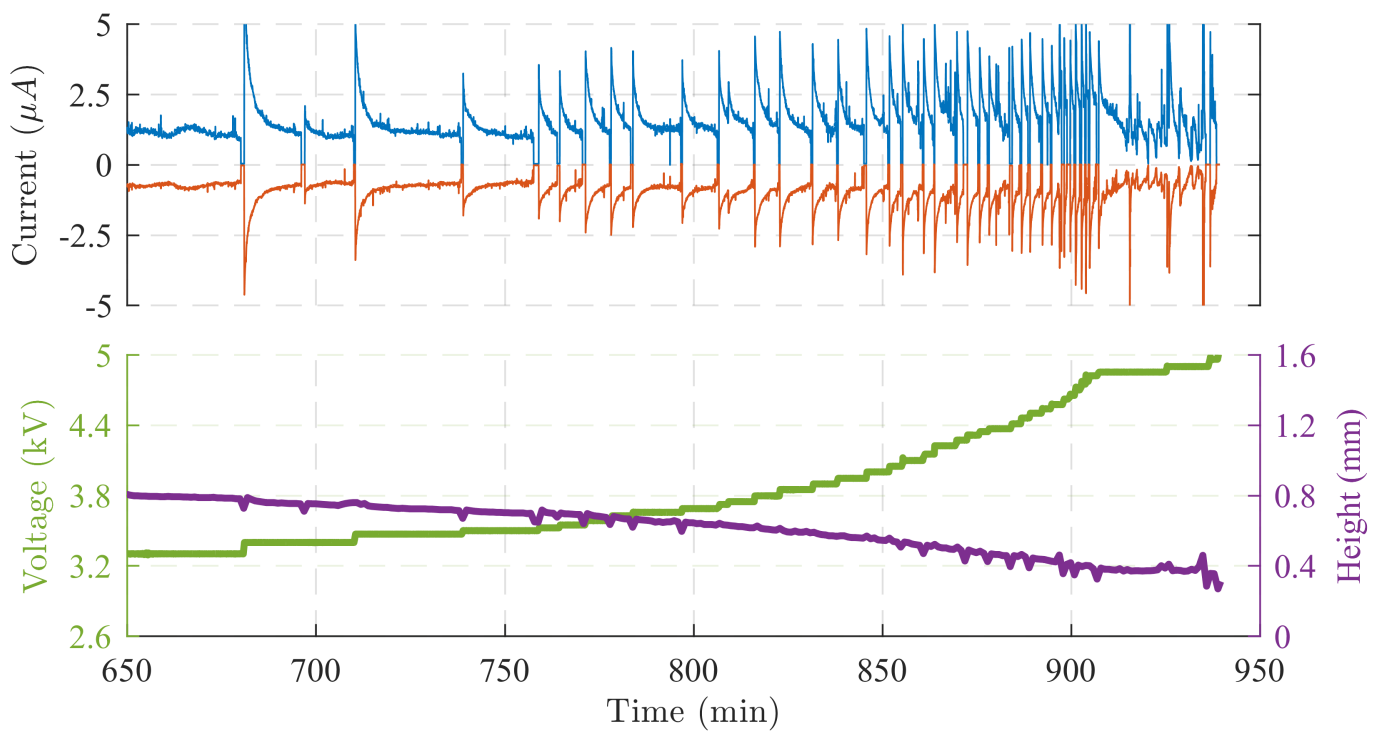

Figure 5.11: Sample shutdown (terminal phase) of electrospray source telemetry. The source will periodically self-extinguish, and the extraction potential is increased slowly in response to restart emission. This process is repeated until the extraction potential reaches the supply maximum of $\pm 5 \mathrm{kV}$ or when the fluid is depleted.

During the constant voltage phase of the emission telemetry, the source to maintain emission at a constant voltage while experiencing a relatively large increase in the apexto-extraction electrode spacing. Such behavior may not seem intuitive since increased spacing decreases the electric field. However, the Prewett and Mair ${ }^{74}$ model for apex field strength shows that the apex electric field is related to the applied voltage $V$, apex radius $R_{c}$, and separation distance between the apex and extractor, $d$, through the following relation:

$$
E_{\text {tip }}=\frac{2 V / R_{c}}{\ln \left(2 d / R_{c}\right)}
$$

In Eq. (5.1), the apex field is proportional to the natural log of the separation distance: $E_{a} \propto 1 / \ln (d)$, therefore the apex field is only weakly dependent on $d$. A plot of Eq. (5.1) is presented in Figure 5.12 where it can be observed that rather large changes in the extraction distance $d$, only a relatively small decrease arises in the electric field at the apex. The separation distance observed during the constant voltage portion of the telemetry 
increased from 1.55 and $2.05 \mathrm{~mm}$. In this range, the model predicts a reduction in apex field strength of approximately 2 percent, assuming a constant $V$ and $R_{c}$. Therefore, a passively fed emitter operating at a voltage only a few percent higher than onset can maintain emission while changes in height resulting fluid depletion results in relatively large increases extraction distance.

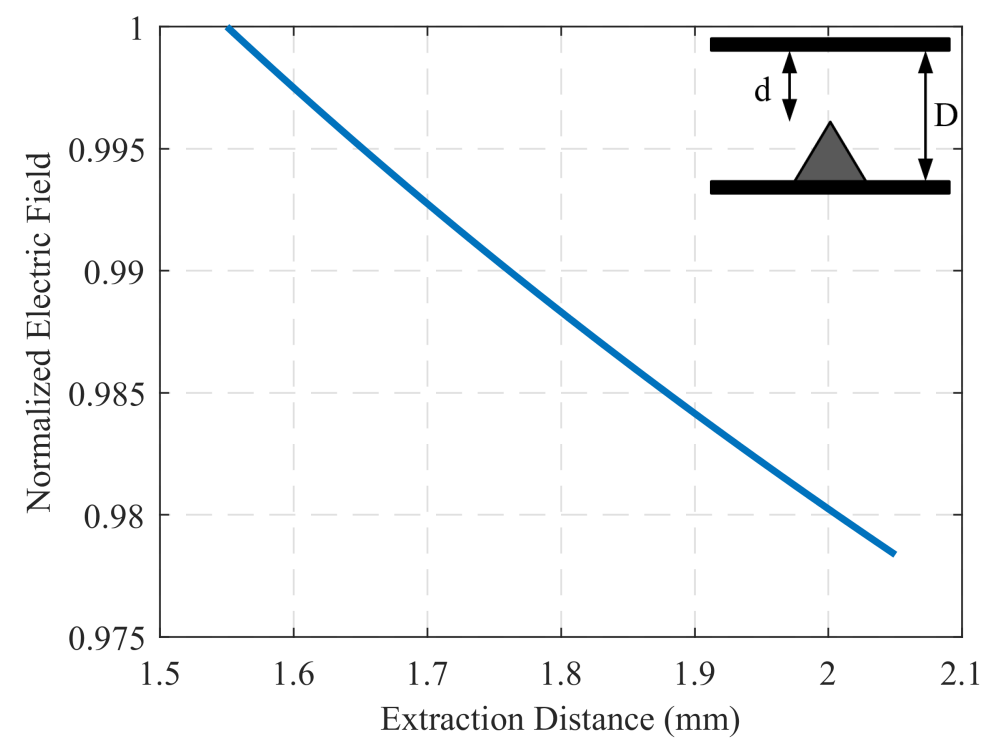

Figure 5.12: Normalized apex electric field using Prewett and Mair ${ }^{74}$ approximation for apex field strength and assuming constant apex radius. $(D=\mathbf{2 . 8 5} \mathbf{~ m m}, \mathrm{V}=\mathbf{2 0 0 0}$ $\mathrm{V}$, and $\mathrm{Ra}=10 \mathrm{~nm}$ ).

\subsection{Influence of Peak Sharpness on Emission Behavior}

While filling the fluid reservoir with ionic liquid ferrofluid, the positioning of the capillary needle relative the reservoir had a considerable impact on the final meniscus profile and ultimately emission behavior. The infusion methodology, presented in Figure 5.13 , controlled the contact line and contact angle of the meniscus - prompting the final fluid to assume one of two meniscus shapes. The first solution, (a) in Figure 5.13, yielded a tall peak with a small apex radius, while the second method (b) resulted in a short peak with a large apex radius. All observed peaks fell into either of these two categories and intermediate solutions were not observed. The magnetic field was not found to have a significant impact on sharpness in the range investigated. 

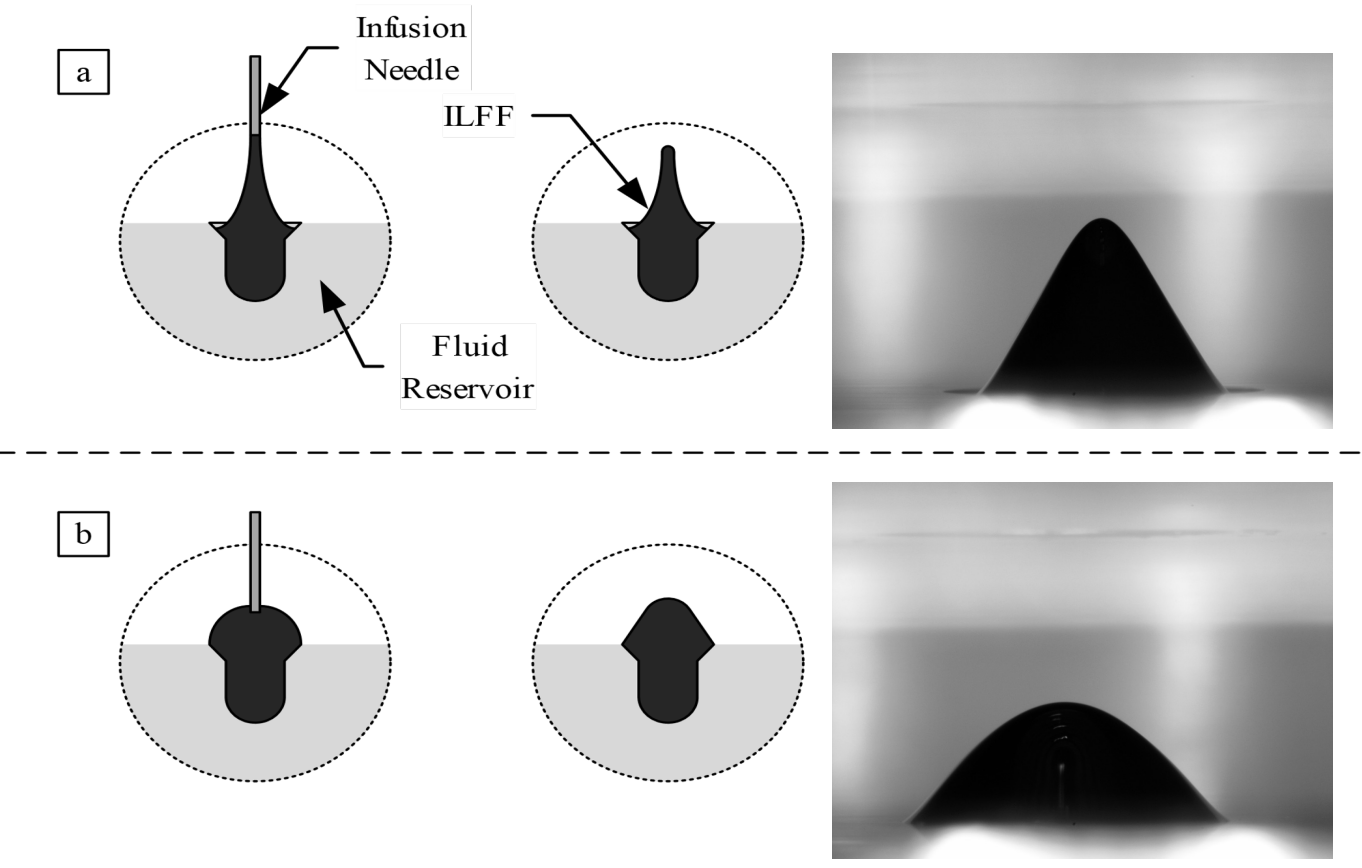

Figure 5.13: Capillary needle position dependence on final meniscus geometry. In both cases, $14 \mu \mathrm{L}$ of ferrofluid was delivered. In case (a) the needle position was raised while infusing the fluid. The gap between the needle and reservoir stretches the fluid, resulting in a concave contact angle and small apex radius. In case (b) the needle was placed too close to the fluid reservoir - resulting in a convex contact angle yielding a large apex radius.

The two menisci solutions demonstrated considerably different electrospray behaviors. The smaller apex corresponded with a low emission current, low mass flow rate, lower onset voltage, and prolonged periods of emission with a constant extraction voltage. The larger apex menisci demonstrated nearly opposite behavior, with high currents, high mass flow rates, and could only maintain emission for short durations without an increase in extraction potential. The emission behavior of the large apex radius menisci closely resembled the shutdown (terminal phase) of operation presented in Figure 5.11 during the entirety of emission.

A large and small apex meniscus solution of the same volume were formed under the same magnetic field and emitted for comparison. The two menisci are shown in Figures 5.14 and 5.15. Emission metrics are presented in Table 5-2. 


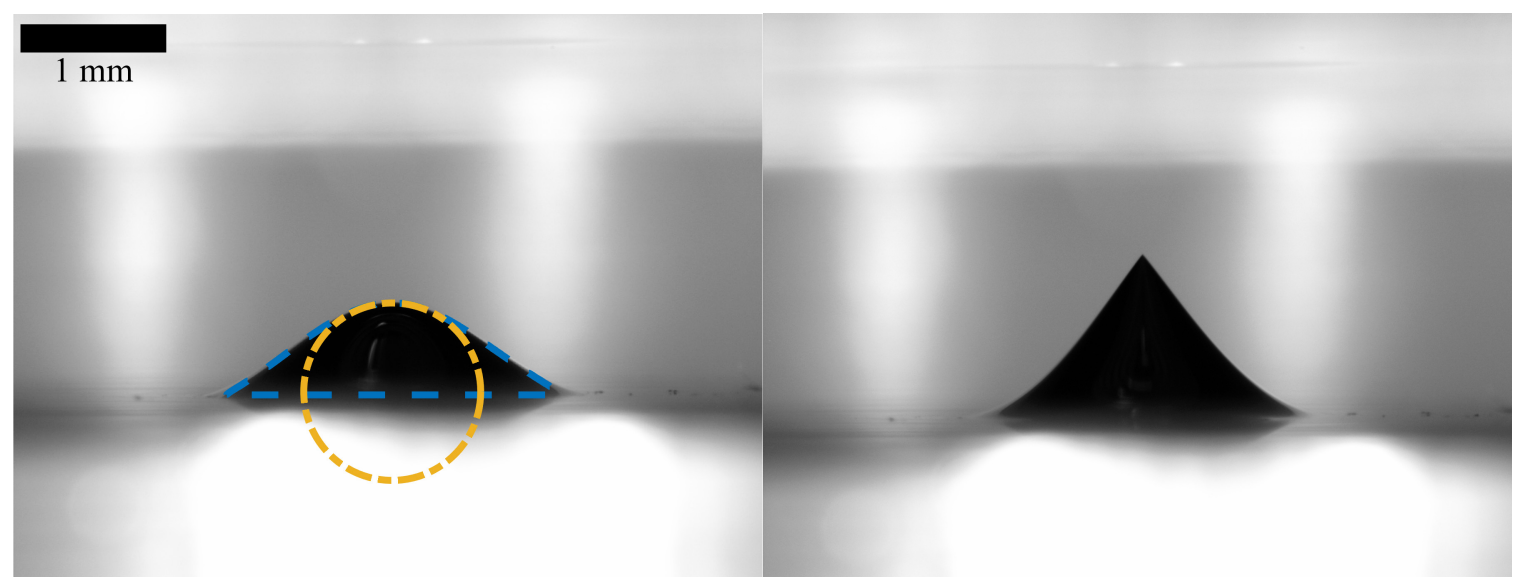

Figure 5.14: Large apex radius solution. (Left) Apex with magnetic field only (Right) Apex immediately after onset.

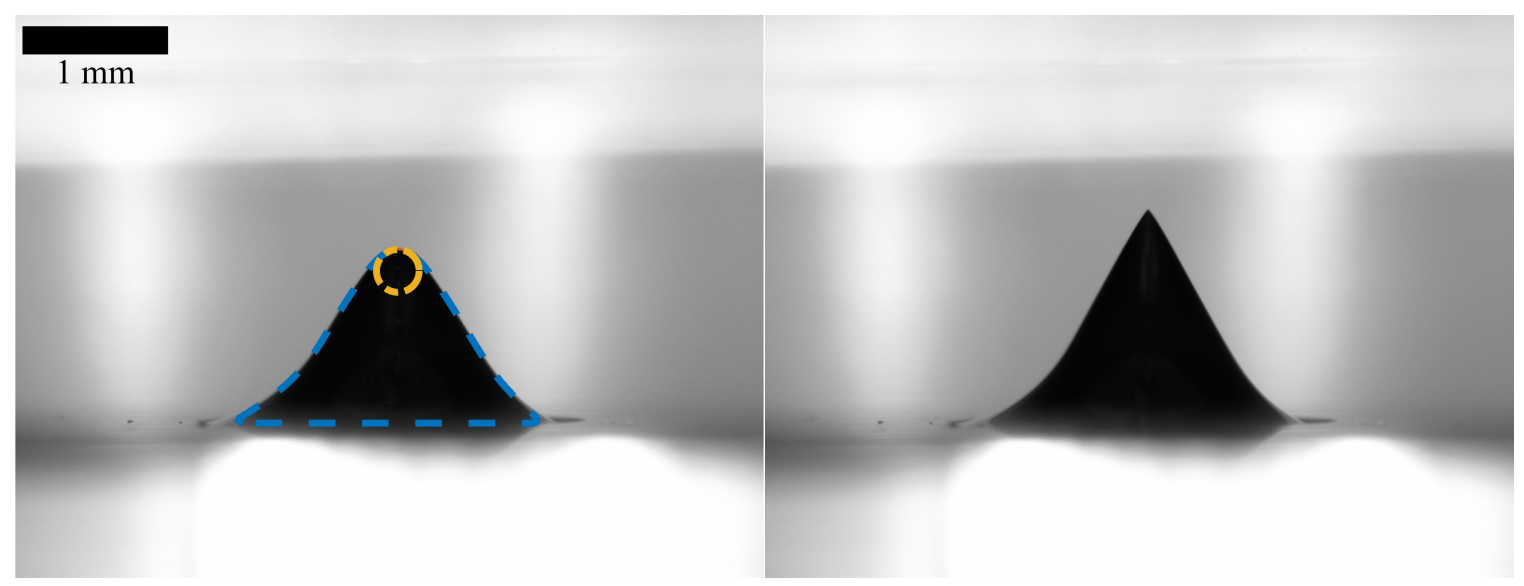

Figure 5.15: Small apex radius solution. (Left) Apex with magnetic field only (Right) Apex immediately after onset.

The following metrics were utilized to compare the influence of apex radius on emission behavior:

- Apex Radius: Estimated by curve fitting a circle to the apex of the normal-field instability with no applied electric field.

- Average Mass Flow: Estimated by time-averaging the mass flow rate while emitting.

- Average Emission Current: Estimated by time-averaging the emission current.

- Voltage Stability: Longest duration at a constant voltage without selfextinguishing. 
Table 5-2: Comparison of emission behavior for small and large apex radius menisci. Both studies were conducted with $14 \mu \mathrm{L}$ of ILFF infused into the fluid reservoir under 495 G magnetic field.

\begin{tabular}{l|c|cc}
\hline & & \multicolumn{2}{|c}{ Test } \\
\cline { 3 - 4 } & & 1 & 2 \\
\hline \hline Apex Radius & $(\mathrm{mm})$ & 0.61 & 0.15 \\
Average Mass Flow Rate & $(\mathrm{ng} / \mathrm{s})$ & 122.9 & 22.8 \\
Average Emission Current & $(\mu \mathrm{A})$ & 3.46 & 0.75 \\
Voltage Stability & $(\mathrm{hr})$ & 0.25 & 11.9 \\
Onset & $(\mathrm{kV})$ & 3.3 & 2.7 \\
Test Duration & $(\mathrm{hr})$ & 2.35 & 16.7 \\
\hline
\end{tabular}

In a process that will be discussed in more detail in Section 5.4.1, the flow rate for passively driven electrospray sources is determined by the excess voltage, or energy, above that required to maintain emission. While studying emission from pressure fed sessile droplets on nonwetting dielectric surface, Lozano, Martínez-Sánchez, and Lopez-Urdiales observed that once emission was achieved, the extraction potential could be decreased by several hundred volts before emission would terminate. ${ }^{96}$ Lozano et al. suggested that the energy necessary to form the structure was higher than necessary to sustain emission. Along similar lines, it is possible that the excess energy available during emission from the large apex is greater than the small apex solution. This energy difference drives the observed higher flow rate.

The primary interest of this research is to analyze the electric propulsion potential of ionic liquid ferrofluid electrospray. The emission behavior presented above in Table 5-2 for the small apex meniscus solution is more promising for this application as a result of the higher emission mass-to-current ratio. Therefore, all subsequent emission studies presented in this report will be limited to the small apex meniscus solution. 


\subsection{Long Duration Emission of a Single Emitter}

A total of three long duration test were conducted with a magnetic field of 321,388 , and 495 gauss. For each test, the source was operated in the manner described in Section 5.2.5. Periodic profiles of the source showing the evolution of the fluid meniscus as the fluid volume is depleted is shown in Figure 5.16. Telemetry containing emission current, extraction voltage, peak height, peak volume, mass flow rate, and charge-to-mass ratios are presented in Figures 5.17 through 5.22 for the three magnetic field strengths investigated. For the emission telemetry presented in Figure 5.21, a slow rise and decay in the emission current can be observed between 300 and 700 minutes in the emission telemetry. It is believed this is a result of the extraction potential being considerably higher than what was necessary to maintain emission. For this test, the start-up voltage was estimated to be 3372 volts based on post-test analysis of the telemetry while the extraction potential during the voltage mode for this test was 3720 volts.

In the same figure, it can be observed that the emission current terminated at 1000 minutes and remained off for approximately 180 minutes. This is a consequence of the emitter self-extinguishing during overnight emission. 


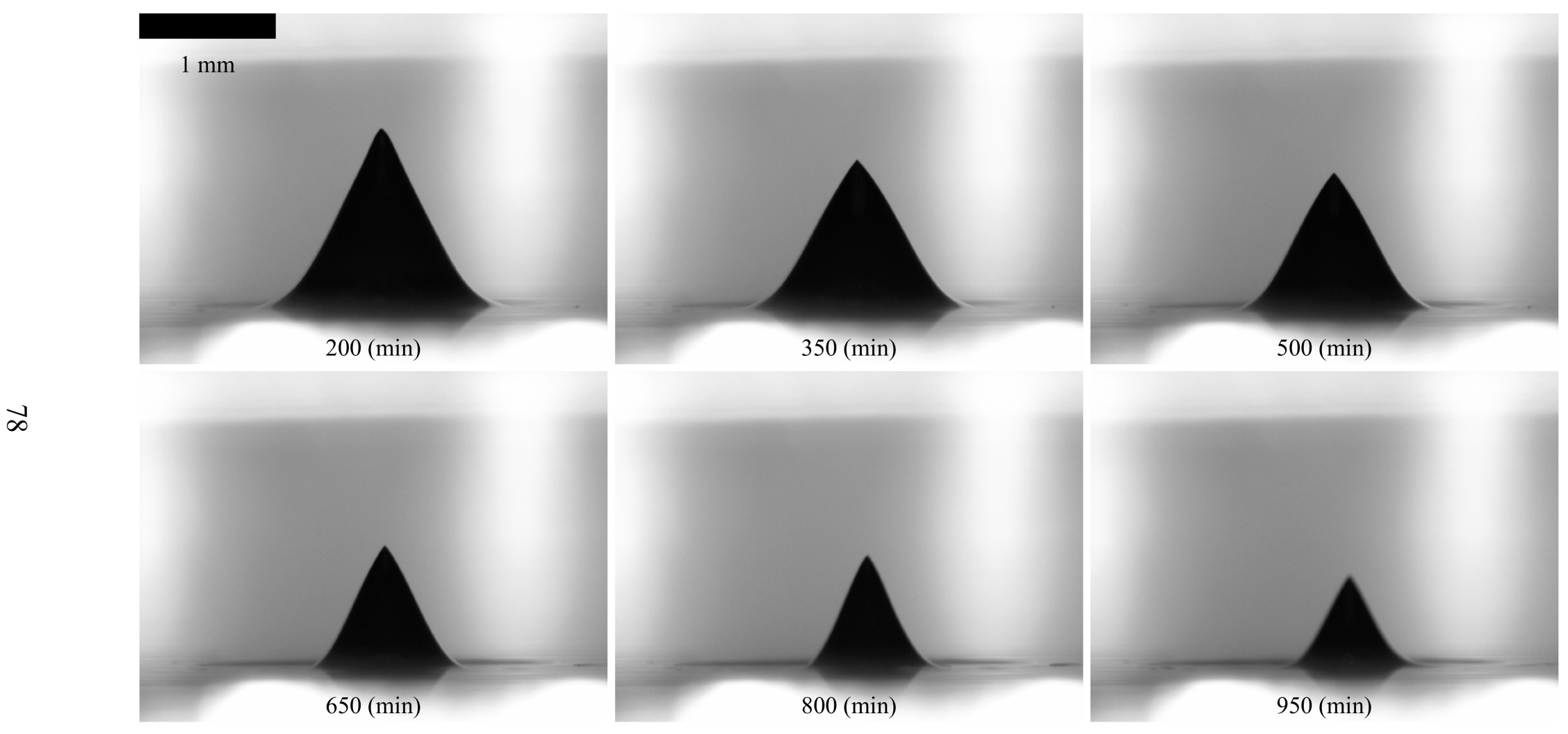

Figure 5.16: Fluid meniscus evolution during emission under a 495 G magnetic field. Extraction potential remained 

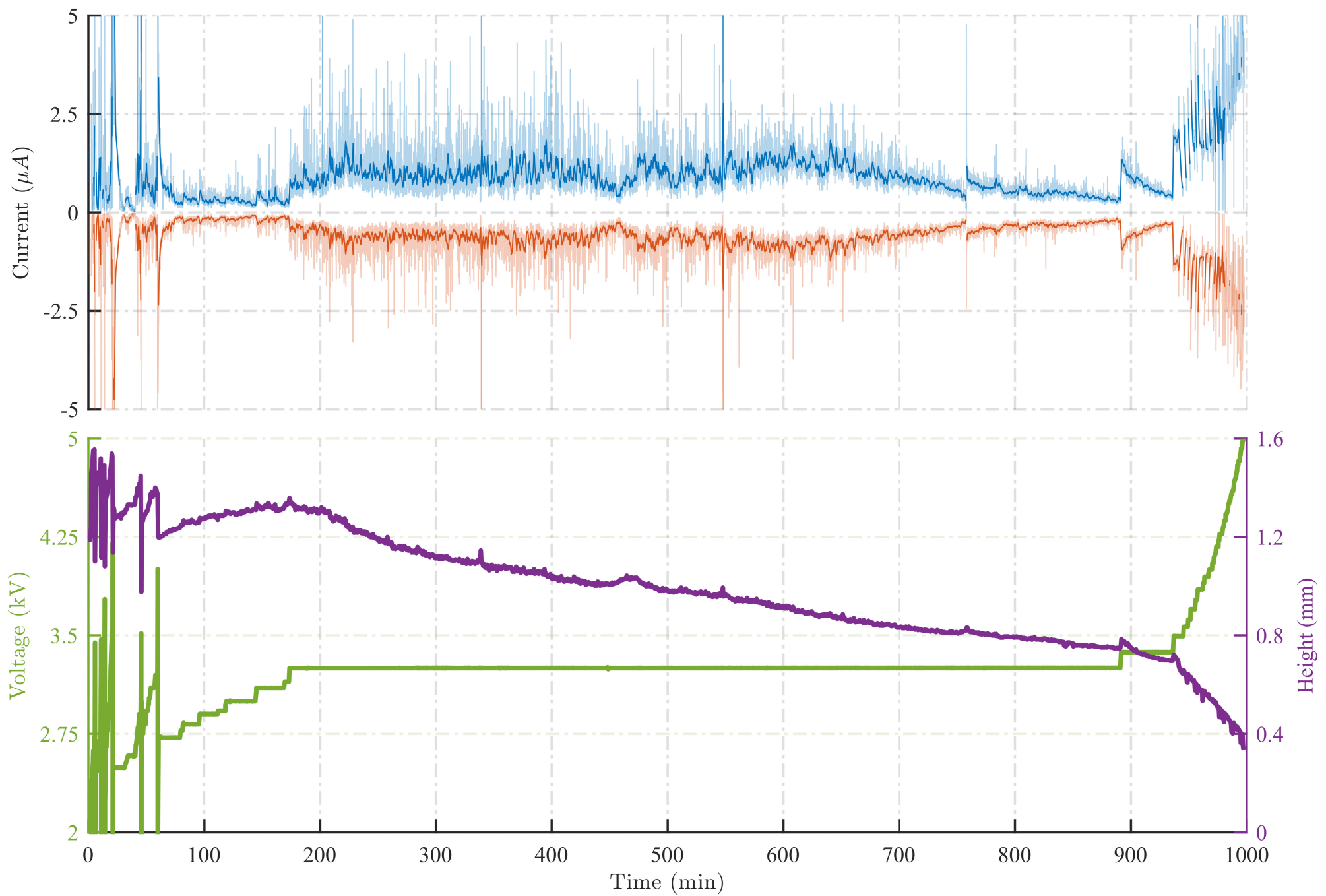

Figure 5.17: (Part 1) Telemetry for an entire electrospray emission test. (30 second moving average is overlayed, $B=495$ 

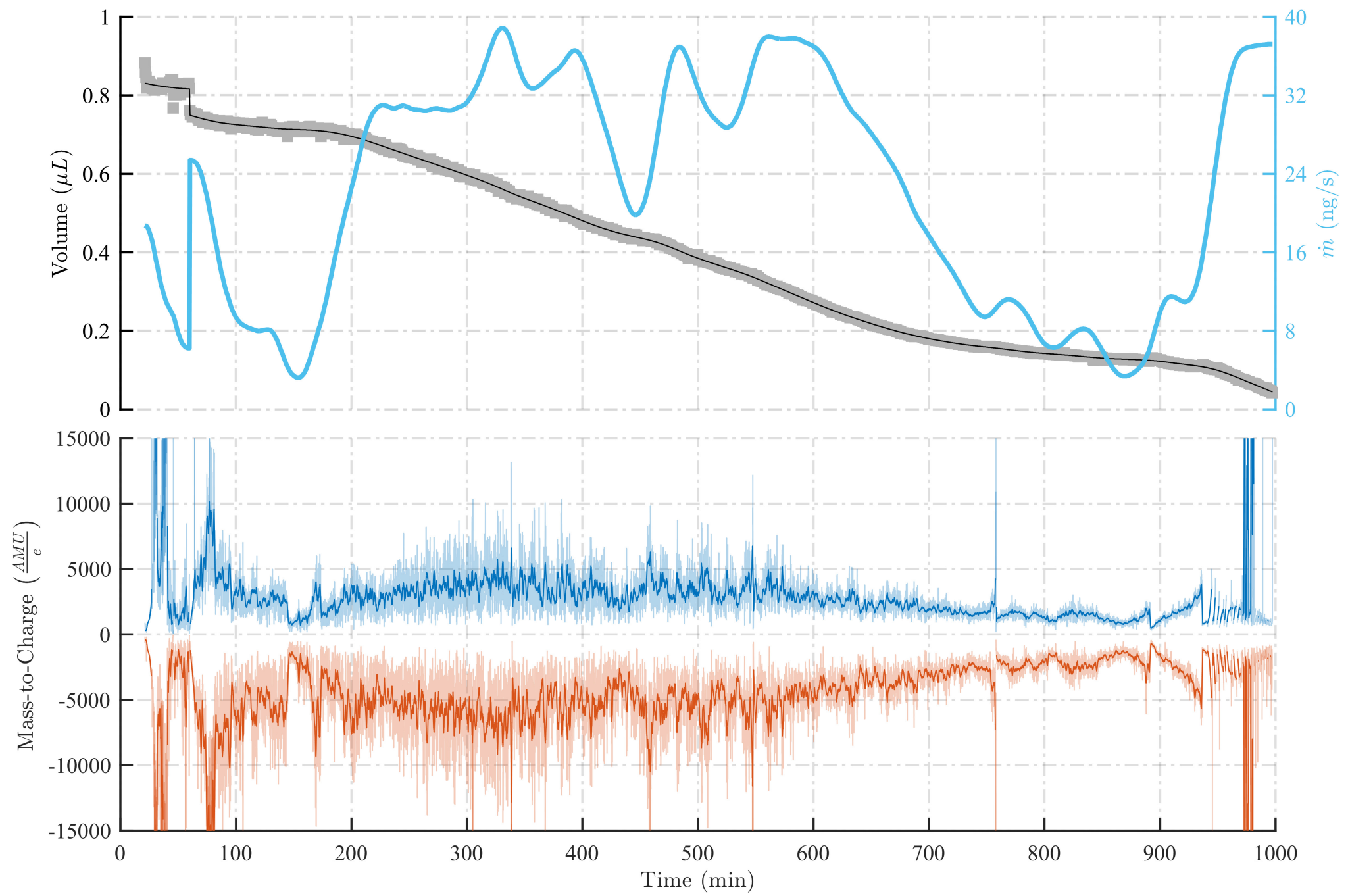

Figure 5.18: (Part 2) Telemetry for an entire electrospray emission test. ( 30 second moving average is overlaved, $B=495$ 

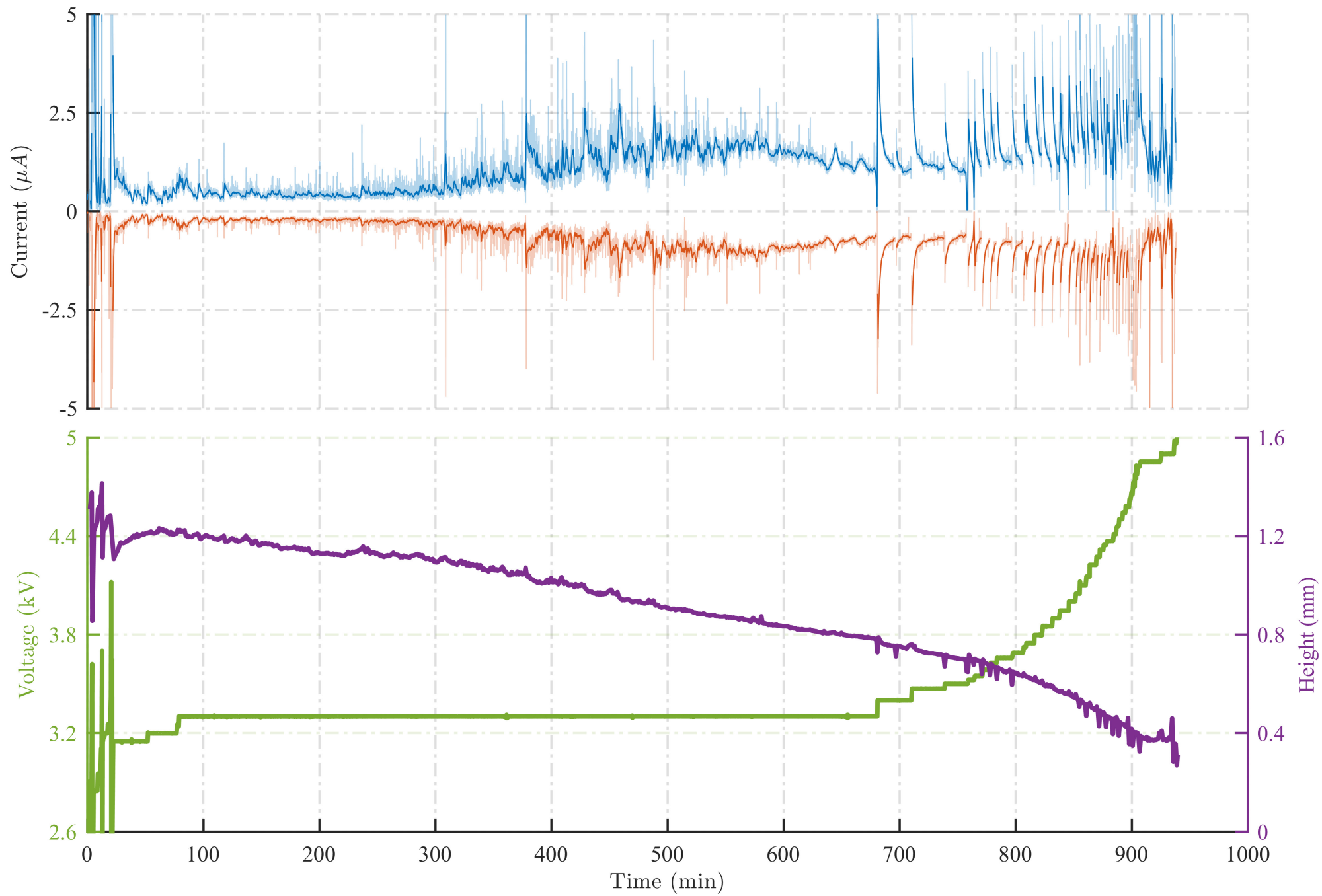

Figure 5.19: (Part 1) Telemetry for an entire electrospray emission test. (30 second moving average is overlayed, $B=388$ 


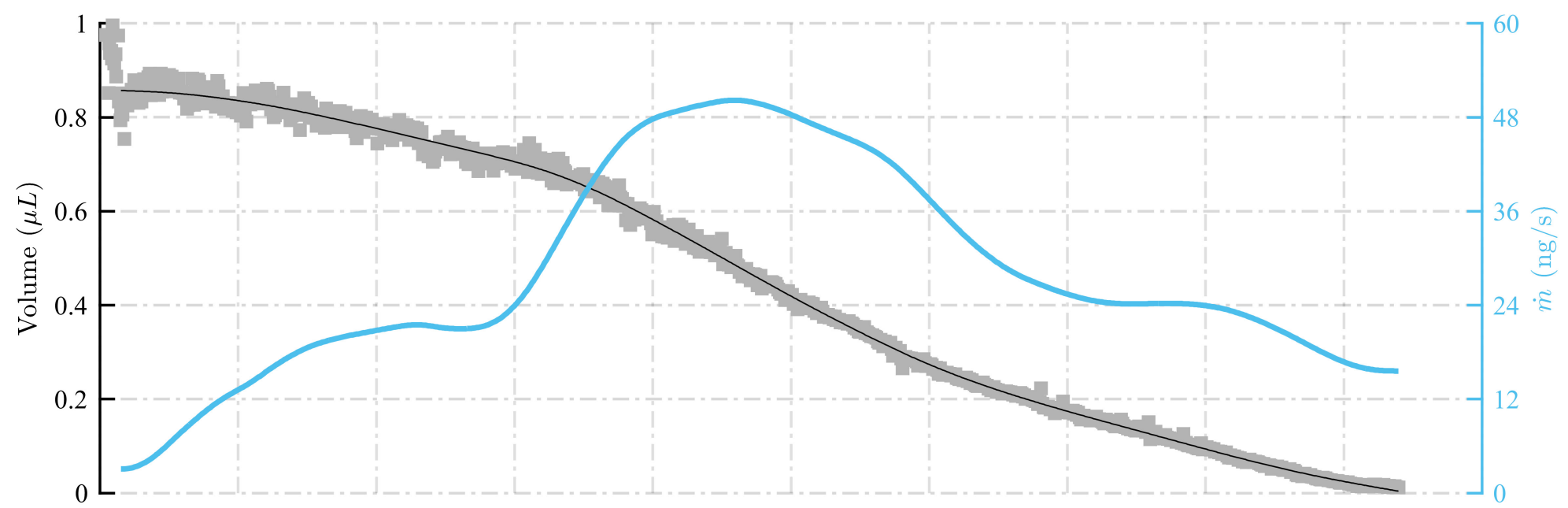

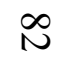

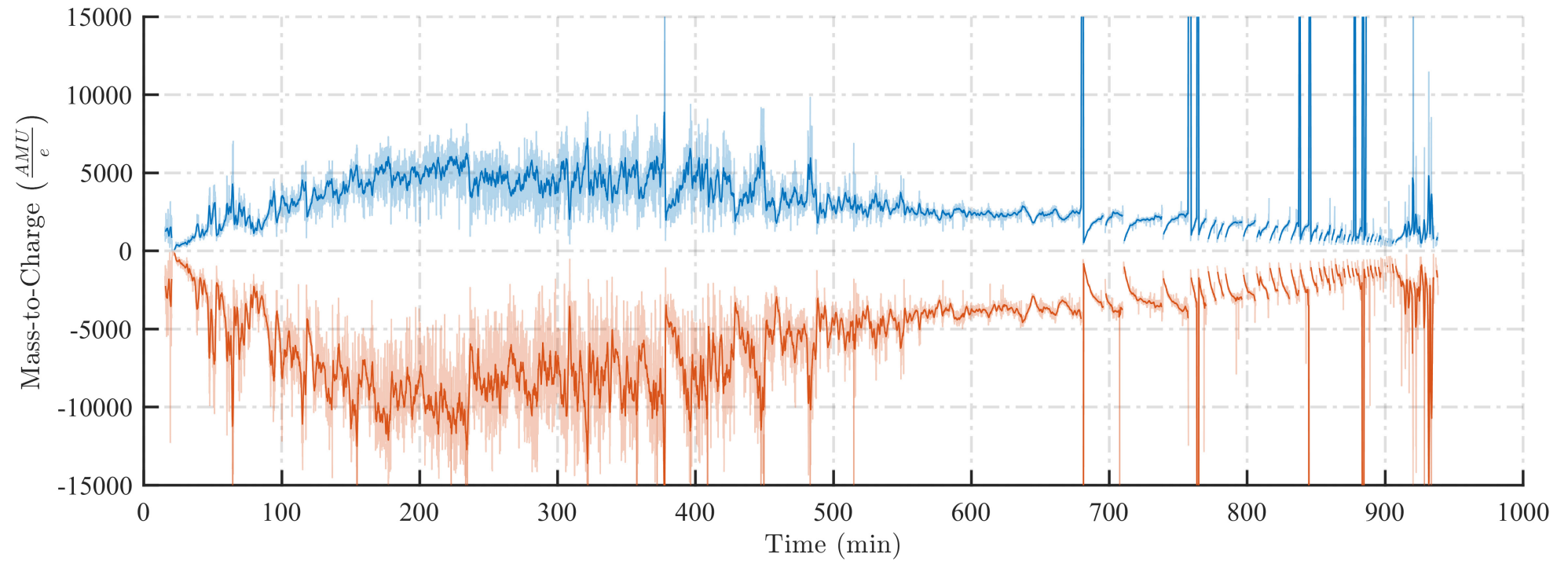

Figure 5.20: (Part 2) Telemetry for an entire electrospray emission test. (30 second moving average is overlayed, $B=388$ 

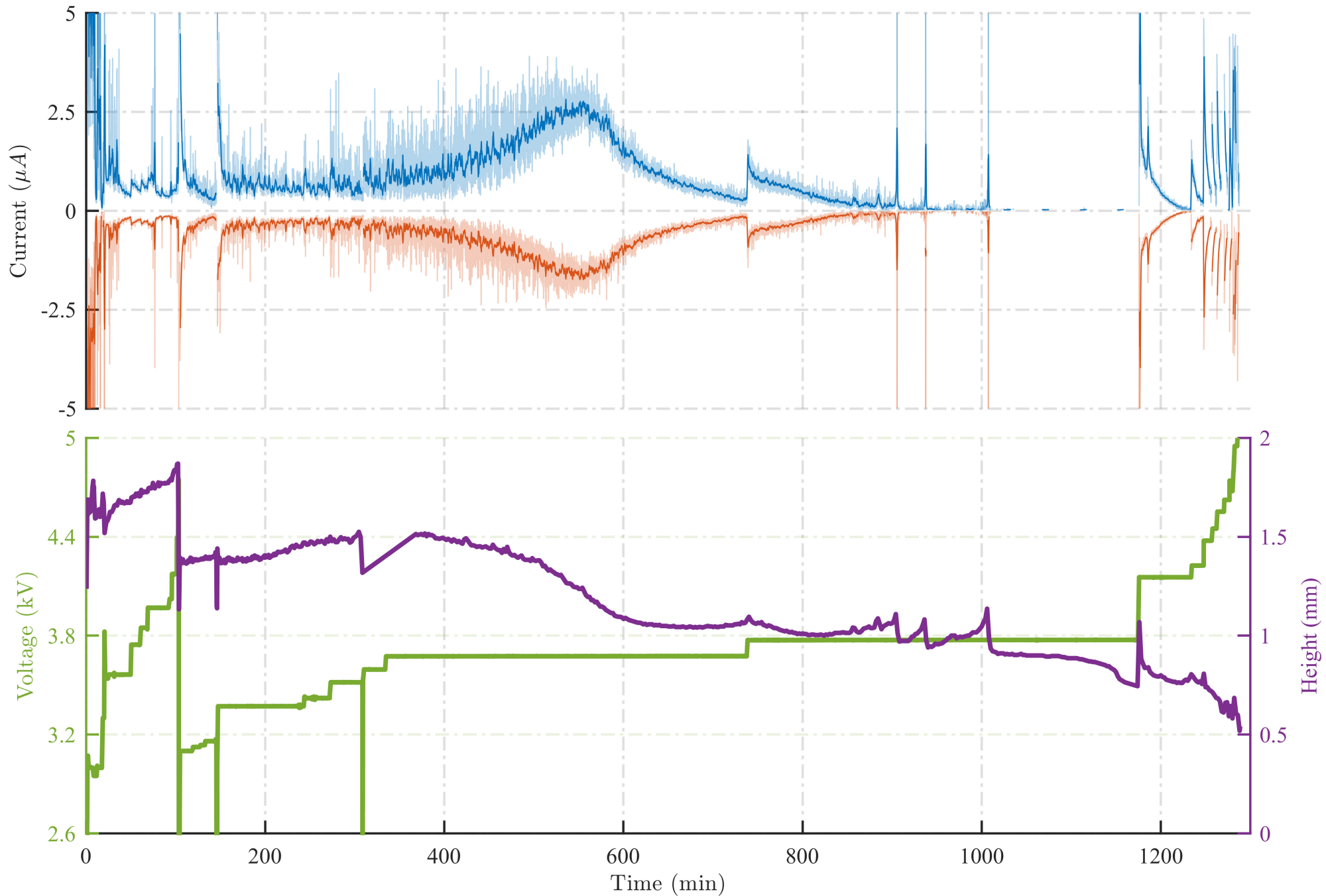

Figure 5.21: (Part 1) Telemetry for an entire electrospray emission test. (30 second moving average is overlayed, $B=321$ 


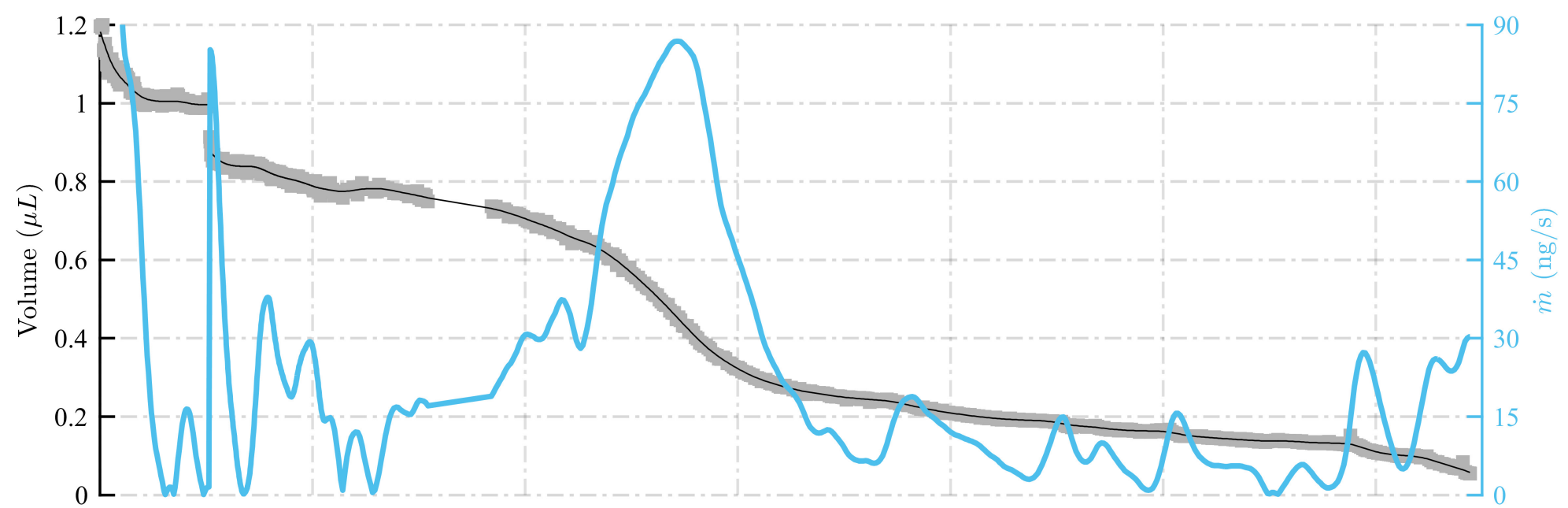

$\stackrel{\infty}{+}$

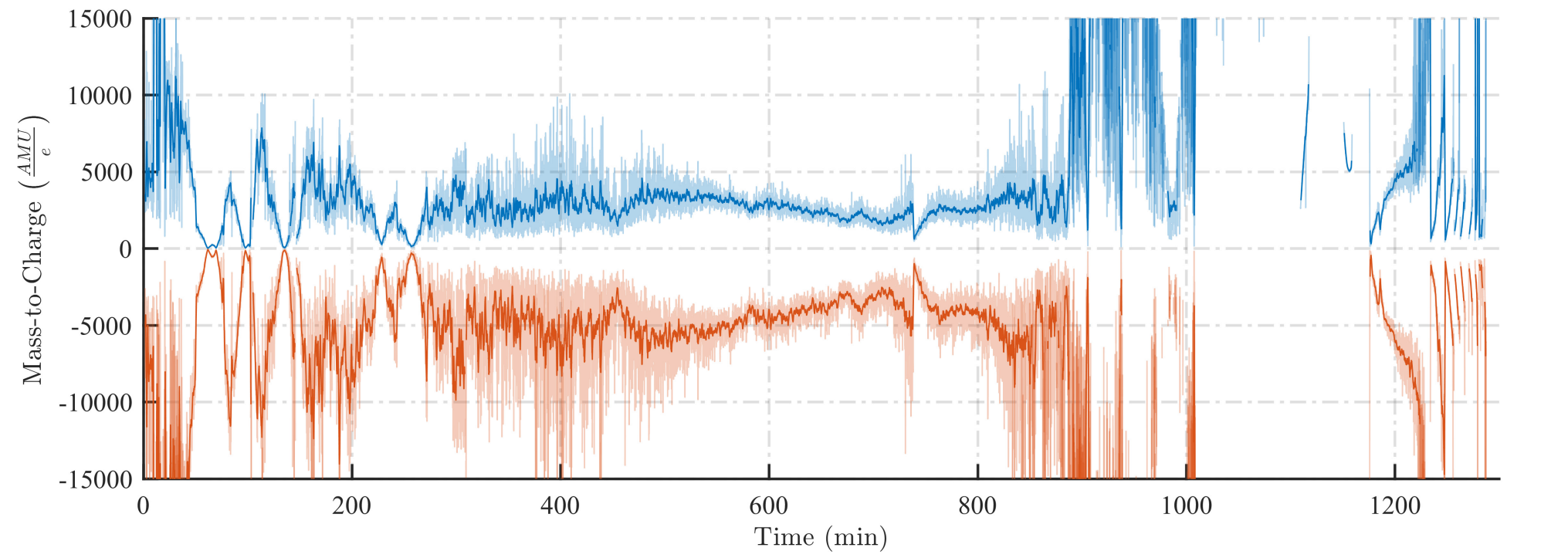

Figure 5.22: (Part 2) Telemetry for an entire electrospray emission test. (30 second moving average is overlaved, $B=321$ 
In all cases, more current was emitted in the positive polarity relative to the negative polarity. A current balance previously presented in Figure 5.7 in Section 5.2.3 shows that the discrepancy between emitted and collected current in both the positive and negative polarity is less than 15 percent. Consequently, secondary electron emission is not believed to be the source for this imbalance. A mismatch between the emitter current for each polarity is not unique to this work and has been observed previously for an electrospray thruster operating with the ionic liquid EMI-IM by Courtney et al. ${ }^{25}$ A ratio of emission currents for these studies is shown in Figure 5.23 where the ratios converged to a value of 1.6 after decaying over several hours from a peak ranging between 1.9 and 2.2. The change in the ratio of emitter currents is suggestive of a change in the emission dynamics of the source, Unfortunately, no changes could be observed in the emission parameters which could be attributed to this trend.

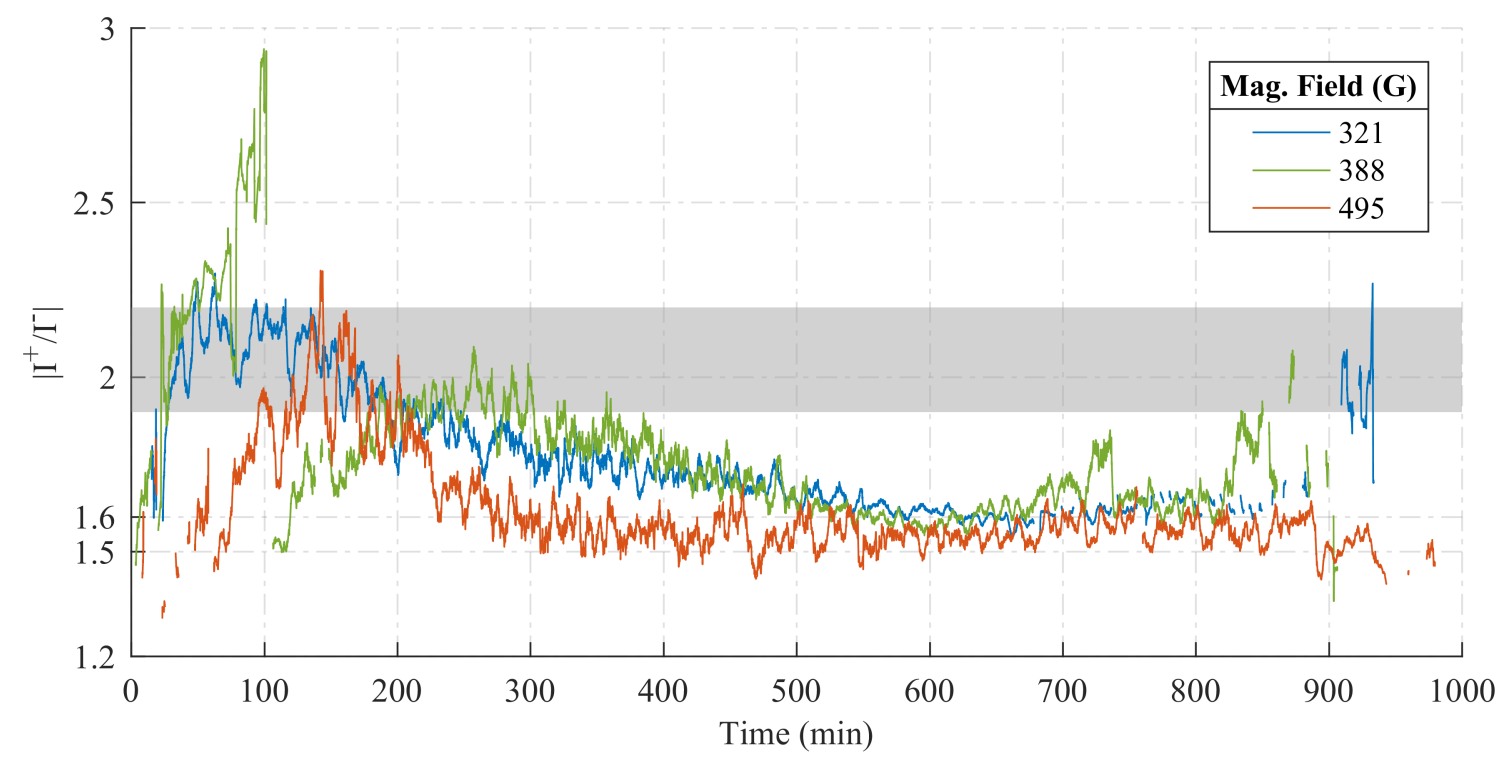

Figure 5.23: Ratio of positive and negative emission currents. The ratio converges on an average of 1.6. A 120-second moving average was applied to both currents before calculating the ratios.

\subsubsection{Mass Flow Rate}

The average flow rates were calculated during the constant voltage portion of the emission telemetry presented in the previous section. These averages are compiled and 
shown below in Table 5-3. The average mass and volumetric flow rates for the three tests is $28 \mathrm{ng} / \mathrm{s}$ and $15.5 \mathrm{pL} / \mathrm{s}$, respectively.

Table 5-3: Average emission flow rates calculated from the net volume change during the constant voltage phase of emission. $(\rho=1.815 \mathrm{~g} / \mathrm{mL})$

\begin{tabular}{ccc}
\hline Mag. Field & $\begin{array}{c}\text { Avg. Mass } \\
\text { Flow Rate } \\
(\mathrm{G})\end{array}$ & $\begin{array}{c}\text { Avg. Volumetric } \\
\text { Flow Rate } \\
(\mathrm{pL} / \mathrm{s})\end{array}$ \\
\hline \hline 321 & 32.8 & 18.1 \\
388 & 26.6 & 14.7 \\
495 & 24.8 & 13.7 \\
\hline Avg. & 28.1 & 15.5 \\
\hline
\end{tabular}

For an ionic liquid ferrofluid normal-field electrospray source, a reasonable hypothesis could have been made that the lack of hydraulic resistance combined with the entire emitter structure being composed of fluid would result in the source exhibiting an uncontrollably high flow rate. In fact, quite the opposite was observed. By comparison, minimum flow rates by Terhune using a $75-\mu \mathrm{m}$ inner diameter capillary needle emission source were on the order of 350 to $500 \mathrm{ng} / \mathrm{s}$ (approximately 200 to $500 \mathrm{pL} / \mathrm{s}$ ). ${ }^{31}$ Madden et al. performed magnetoelectrospray experiments using a sulfolane-based ferrofluid with ethyl ammonium nitrate added to control conductivity. Using a $40 \mathrm{uL}$ inner diameter capillary needle and a ferrofluid with a conductivity of $0.3 \mathrm{~S} / \mathrm{m}$, Madden observed flow rates in a 295 gauss uniform magnetic field as low as $75 \mathrm{pL} / \mathrm{s}^{36}$

Notably, both authors observed a lower minimum flow rate for a magnetized fluid relative to unmagnetized spray. Madden ${ }^{36}$ observed a 30 percent reduction in minimum flow with a 295 gauss magnetic field while Terhune ${ }^{31}$ observed up to a 40 percent reduction with a 200 gauss field. In his work, Terhune sprayed ferrofluids of dissimilar nanoparticle concentrations and positive correlation was observed between nanoparticle concentration and percent reduction in minimum low rate. The mechanism which reduces the minimum stable flow rates for magnetoelectrospray sources remains unknown. Madden et al. put 
forward a hypothesis that the magnetic stresses may partially compensate the capillary stress, reducing the surface charge necessary to form an emitting tip. ${ }^{36}$ The mechanism which is permitting lower flow rates in capillary needles likely transfers to normal-field electrospray sources. However, since electrospray emission cannot be achieved from a normal-field instability without a magnetic field, this mechanism is best investigated in capillary sources where emission can be achieved in an unmagnetized fluid.

To analyze the difference in flow rates between the normal-field and capillary sources, two factors are worth noting. First, the normal-field emission studies did not actively seek to minimize flow rate. Rather, the extraction potential was set to obtain a positive polarity emission current of around $0.75 \mu \mathrm{A}$. In all likeliness, a lower flow rate could have been obtained by reducing the extraction potential. Second, the emission studies by Terhune ${ }^{31}$ and Madden et al., ${ }^{36}$ utilized pressure fed capillary to replenish fluid to the Taylor cone. The normal-field electrospray source has no external mechanism to control fluid delivery in this manner. Instead, fluid flow to the emission site of the normal-field source are driven purely by internal pressure gradients within the fluid, behavior which more closely resembles that of passively fed electrospray sources. Courtney and Shea note that in passively fed electrospray sources, emission current and mass flow are controlled solely by the applied extraction potential. ${ }^{97}$

In the Ph.D. dissertation of Perez-Martinez a method for determining flow impedance from emission parameters was proposed for a passively fed source. ${ }^{98}$ For this model, consider the case of a traditional passively fed electrospray source, such as a porous needle, where there is no magnetic stresses. The electrostatic stress at the apex, $P_{E}$, is equal to the stress required to overcome the capillary pressure, plus an excess amount $\Delta p$.

$$
P_{E}=\frac{2 \gamma}{R_{c}}+\Delta p
$$

Where the electrostatic stress at the apex is the same as that presented Eq. (2.9):

$$
P_{E}=\frac{1}{2} \varepsilon_{0} E_{n}^{2}
$$


At the startup voltage, $V_{\text {start }}$, the excess stress is zero and only materializes at voltages $V$ that are of magnitude $\Delta V$ above the startup voltage. The startup voltage can be estimated based on the Prewett \& Mair model, presented in Chapter 4, and is restated here for convenience: ${ }^{74}$

$$
V_{\text {start }}=\ln \left(\frac{2 d}{R_{c}}\right) \sqrt{\frac{\gamma R_{c}}{\varepsilon_{0}}}
$$

where $R_{c}$ is the apex radius the meniscus and $d$ is the distance between the apex and the extractor. During the derivation of this model, the electric field at the apex was derived to be: $:^{74}$

$$
E_{\text {tip }}=\frac{2 V / R_{c}}{\ln \left(2 d / R_{c}\right)}
$$

Note: Eq. (5.5) is a reduction of a more vigorous model made under the assumption that $R_{c} \ll d$. From equations (5.2) through (5.5), the value of the excess pressure $\Delta p$ can be found in terms of the excess voltage $\Delta V$ :

$$
\begin{aligned}
\Delta p & =\frac{2 \gamma}{V_{\text {start }}^{2} R_{c}}\left(V^{2}-V_{\text {start }}^{2}\right) \\
& =\frac{2 \gamma}{R_{c}}\left(\frac{2 \Delta V}{V_{\text {start }}}+\frac{\Delta V^{2}}{V_{\text {start }}^{2}}\right)
\end{aligned}
$$

The flow impedance $Z_{i m p}$ is simply related to the flow rate $Q$ and pressure differential as follows:

$$
Q=\frac{\Delta p}{Z_{i m p}}
$$


The parameters $\Delta p, Z_{i m p}$, and $R_{c}$ are unknown. All other parameters can be measured. Using average tip height from the telemetry, the extractor spacing, $d$, is $1.85 \mathrm{~mm}$. For the trials presented in Table 5-3, the resulting impedances range from $1.7 \times 10^{14}$ to $8.7 \times 10^{14}$ $\mathrm{Pa} \cdot \mathrm{s} / \mathrm{m}^{3}$. For comparison, the same approach to calculate impedance was implemented by Perez-Martinez and a flow impedance of $\sim 4.7 \times 10^{19} \mathrm{~Pa} \cdot \mathrm{s} / \mathrm{m}^{3}$ for a carbon xerogel emitter designed for pure ionic emission. ${ }^{98}$ As part of the Microthrust project, a pressure fed array of microsphere packed capillaries was fabricated with an estimated impedance on the order of $1.5 \times 10^{17} \mathrm{~Pa} \cdot \mathrm{s} / \mathrm{m}^{3}$. The Microthrust thruster was found to operate near the pure ionic regime. ${ }^{99}$ It has been observed that lower flow rate emitters are more prone to emit in the pure ionic regime. Consequently, much of the current effort being performed on electrospray thrusters focuses on passively driven designs which have demonstrated the necessary range of impedances. ${ }^{14,25,98,100-102}$

Clearly, a considerable flow impedance is present in the normal-field electrospray source. However, this impedance is not sufficiently high to yield a pure ionic regime of emission. To further analyze what could be providing this impedance, let us digress temporarily to analyze the cone-jet region and jet diameter. From scaling laws presented by Fernández de la Mora, the radius of the electrospray jet is known to scale with respect to the characteristic radius $r^{*}$ defined as: ${ }^{38}$

$$
r^{*}=\left(\frac{Q \varepsilon_{0}}{K}\right)^{1 / 3}
$$

where $Q$ is the flow rate and $K$ is the fluid conductivity. The jet diameter, $d_{j}$, becomes:

$$
d_{j}=G(\varepsilon) r^{*}
$$

where $G(\varepsilon)$ is a proportionality constant which is a function of the fluid permittivity and must be determined experimentally. Fernández de la Mora notes that the value of $G(\varepsilon)$ is of order unity for all values of $\varepsilon$ for which data is available. ${ }^{42}$ For a flow rate of $15 \mathrm{pL} / \mathrm{s}$ 
and assuming a $G(\varepsilon)$ of 1 , the approximate jet diameter is approximately $12 \mathrm{~nm}$. The nanoscale magnetic particles which compose the ferrofluid are on the order of 10-15 nm. The surfactant coating the particles adds to the particle size, and the measured hydraulic diameter is estimated to be $20-30 \mathrm{~nm} .{ }^{92}$ The volume fraction of the nanoparticles in the colloidal suspension is approximately 7 percent.

The scale of the electrospray jet being on the same order as the particle diameters reveals two relevant questions: (1) does the scale of the hydraulic jet prevent particles from being emitted, and (2) can a bottle-necking of the particles in the cone-jet rejoin create a nanoparticle induced flow impedance. The answer to both of these questions are of interest for understanding the low flow rate. The emission of nanoparticles from the normal-field emission source has not been verified directly. However, buildup of black residue on the collector plate, is very suggestive that particles are being emitted since the carrier fluid EMIM-NTf2 is translucent.

The second question considers the possibility of flow impedance resulting from bottle necking of nanoparticles at the apex. Such an occurrence could restrict the flow until an equilibrium is reached wherein the ratio of nanoparticle in the spray is representative the bulk ferrofluid. The presence and dynamics of this process, which is illustrated in Figure 5.24 , is something that can only be theorized with the available data. 


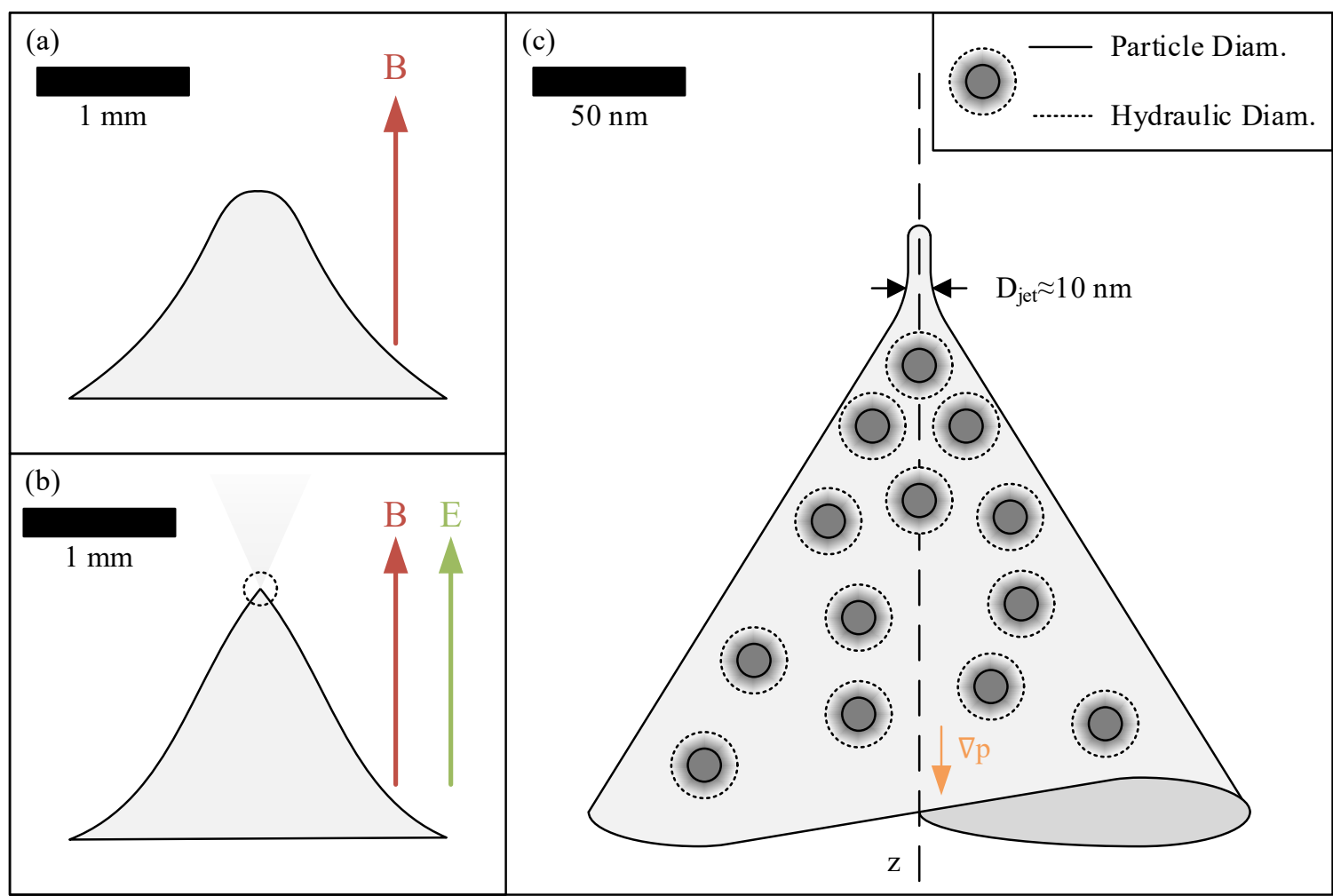

Figure 5.24: (a) Ferrofluid meniscus deformation under only a magnetic field. (b) The addition of an electric field further stresses the fluid until emission is achieved. (c) The apex region of emitting normal-field instability. The hydraulic diameter of the nanoparticles $(20-30 \mathrm{~nm})$ is comparable with the jet diameter $(\approx 10 \mathrm{~nm})$.

\subsubsection{Derived Propulsion Performance Parameters}

In this section, derived propulsion performance results are presented for the emission studies presented previously in Section 5.4. Propulsion metrics for three performance tests are presented in Table 5-4. These propulsion parameters were calculated during the constant voltage portion of the emission telemetry. These parameters were calculated in terms of average emission current $\bar{I}$, the average mass flow rate $\dot{\bar{m}}$, and the emission current $V$. These parameters were calculated as follows. First, the average emission current in each polarity was determined:

$$
\bar{I}^{+,-}=\frac{1}{t_{2}-t_{1}} \int_{t_{1}}^{t_{2}} I^{+,-} d t
$$

The combined average emission current becomes: 


$$
\bar{I}=\frac{1}{2}\left(\bar{I}^{+}+\bar{I}^{-}\right)
$$

The average combined mass flow rate of the emitter is:

$$
\dot{\bar{m}}=\frac{1}{t_{2}-t_{1}} \int_{t_{1}}^{t_{2}} \dot{m} d t
$$

Finally, the average mass-to-charge ratio can now be calculated as follows:

$$
m / q=\frac{\dot{\bar{m}}}{\bar{I}}
$$

From Equations (1.3) through (1.6), the performance parameters in Table 5-4 were calculated.

\begin{tabular}{|c|c|c|c|c|c|}
\hline & \multicolumn{3}{|c|}{ Test \# } \\
\hline & & & 1 & 2 & 3 \\
\hline \multicolumn{2}{|c|}{ Magnetic Field } & (G) & 495 & 388 & 321 \\
\hline \multicolumn{2}{|c|}{ Emission Duration } & (hr) & 16.7 & 18.4 & 15.6 \\
\hline \multicolumn{2}{|c|}{ Voltage } & $(\mathrm{kV})$ & 3.25 & 3.72 & 3.03 \\
\hline \multirow{7}{*}{ 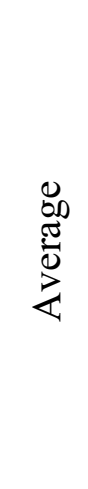 } & Mass Flow Rate & $(\mathrm{ng} / \mathrm{s})$ & 24.8 & 26.6 & 32.8 \\
\hline & Emission Current & $(\mu \mathrm{A})$ & 0.75 & 0.84 & 0.79 \\
\hline & \multirow{2}{*}{ Mass-to-Charge Ratio } & $(\mathrm{kg} / \mathrm{C})$ & $3.31 \times 10^{-5}$ & $3.61 \times 10^{-5}$ & $4.16 \times 10^{-5}$ \\
\hline & & $(\mathrm{AMU} / \mathrm{e})$ & $3.19 \times 10^{3}$ & $3.48 \times 10^{3}$ & $4.01 \times 10^{3}$ \\
\hline & Exhaust Velocity & $(\mathrm{m} / \mathrm{s})$ & $1.39 \times 10^{4}$ & $1.43 \times 10^{4}$ & $1.25 \times 10^{4}$ \\
\hline & Specific Impulse & (s) & 1422 & 1455 & 1278 \\
\hline & Thrust & $(\mu \mathrm{N})$ & 0.346 & 0.380 & 0.412 \\
\hline
\end{tabular}

Table 5-4: Tabulated derived propulsion metrics for long duration emission tests. Average values calculated during constant voltage mode of emission.

Using the average mass-to-charge ratio, the average droplet diameter can be estimated from the Rayleigh limit. The Rayleigh limit is the maximum amount of charge that a droplet can 
carry while remaining stable. Above this limit, the droplet will either form a local electrospray emission site or explosively disassociate through coulombic explosion. The Rayleigh limit is found by setting the electric pressure equal to the capillary pressure:

$$
\frac{1}{2} \varepsilon_{0}\left(\frac{Q_{r}}{4 \pi \varepsilon_{0} r^{2}}\right)^{2}=\frac{2 \gamma}{r}
$$

Where $\left(Q_{r} / 4 \pi \varepsilon_{0} r^{2}\right)$ is the electric field at the surface of a uniformly charged spherical droplet of radius $r$ carrying a net charge $Q_{r}$. Solving for $Q_{r}$ yields the Rayleigh limit:

$$
Q_{r}=8 \pi \sqrt{\gamma \varepsilon_{0} r^{3}}
$$

The mass-to-charge ratio of a droplet becomes:

$$
\frac{m}{q}=\frac{\Phi \rho r^{3}}{6 \sqrt{\varepsilon_{0} \gamma r^{3}}}
$$

In the previous equation, the term $\Phi$ is introduced. This term is the Rayleigh limit charge fraction which permits droplets to be charged to some fraction of their Rayleigh limit - a common observation. Solving for $r$ in Eq. (5.16) yields:

$$
r=\left[\left(\frac{6 m}{\Phi q \rho}\right)^{2} \varepsilon_{0} \gamma\right]^{1 / 3}
$$

The ferrofluid density $\rho$ is $1.815 \mathrm{~g} / \mathrm{mL}$ (Table 4-1, Pg. 36). Assuming a charge fraction of $\Phi=0.5$, which is a reasonable average based on distributions measured by Miller, ${ }^{103}$ the approximate droplet radius is $2.6 \mathrm{~nm}$. A droplet of that size is smaller than the nanoscale magnetic particles present in the fluid, which are on the order of 10-15 nm. As with traditional electrosprays operating in a mixed droplet mode, a bulk of the current is carried by ions while the bulk of the volumetric flow is being carried by larger droplet 
distribution, which carries less current due to the Rayleigh limit. Separate emission species is supported by the angular divergence results presented in the next chapter.

\subsection{Source Sensitivity and Emission Site Dynamics}

The sensitivity of the source to changes in the extraction potential were analyzed at two locations in the typical emission telemetry. The first location, referred to as 'mode-1' was taken during the constant voltage portion. The second mode, 'mode-2' was observed during the terminal phase of testing where it becomes necessary to routinely increase the extraction potential to maintain emission.

To analyze the sensitivity of the source to abrupt changes in extraction potential, the source started in a similar manner as previously presented tests and allowed to emit for 9 hours prior to sensitivity testing. The sensitivity was first tested by applying incremental increases of 50 volts, from 100 to 300 volts for 15 -minute. The extraction voltage was then returned to the initial voltage for 15 minutes prior to the next step. The results of this study, presented in Figure 5.25, show that the source is sensitive to changes in extraction potential yet remains stable. This stability is demonstrated at the 680-minute mark in Figure 5.25 where an $11 \%(300 \mathrm{~V})$ increased in extraction potential, resulted in an emission current increase $224 \%$ which remained relatively constant during the period of excited extraction potential. 

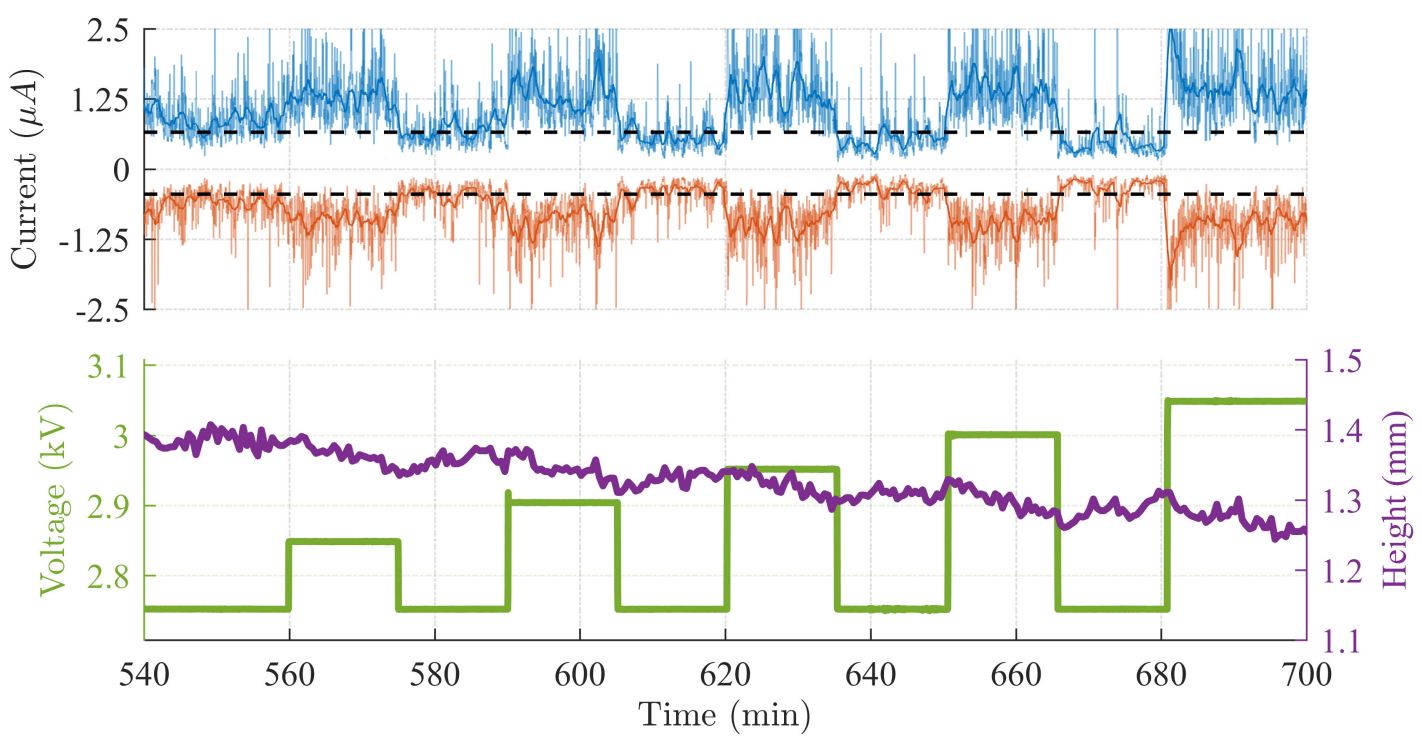

Figure 5.25: Current response to step changes in extraction potential in emission mode-1. (30 second moving average is overlayed)

A voltage ramp was then performed on the emitter while operating in 'mode-1.' To perform this ramp, the extraction potential was incrementally increased while operating in a bipolar emission mode to achieve an average rate of $0.9 \mathrm{~V} / \mathrm{s}$. Emission current and extraction potential with respect to time for this exercise are presented in Figure 5.26. Relating emission current to extraction potential yields IV traces, which are presented in Figure 5.27 for both polarities. A consistently higher emission current for both polarities during the increasing segment suggests hysteresis is present in emission dynamics of the emitter. When the voltage was restored to the initial extraction potential of $3000 \mathrm{~V}$, a $27 \%$ decrease in emission current for both polarities resulted when the initial spike following the voltage restoration is negated. The percent increase in emission current resulting from 
a $300 \mathrm{~V}$ increase in the extraction potential in Figure 5.26, yielded a $227 \%$ increase in emitted current - closing matching the $224 \%$ increase observed when stepping the voltage.

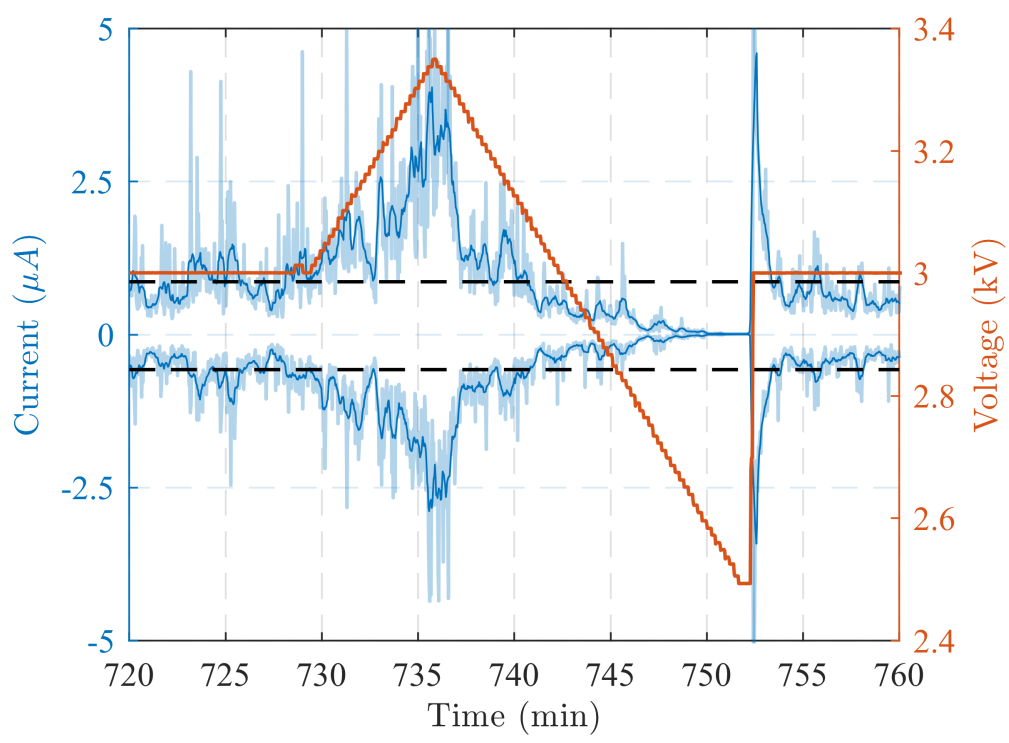

Figure 5.26: Voltage ramp for ILFF electrospray source operating in emission 'mode1.' Extraction potential was changed at a rate of $0.9 \mathrm{~V} / \mathrm{s}$. A 10-second moving average is overlayed to accent trends, but all analyses were performed on the unaveraged data.
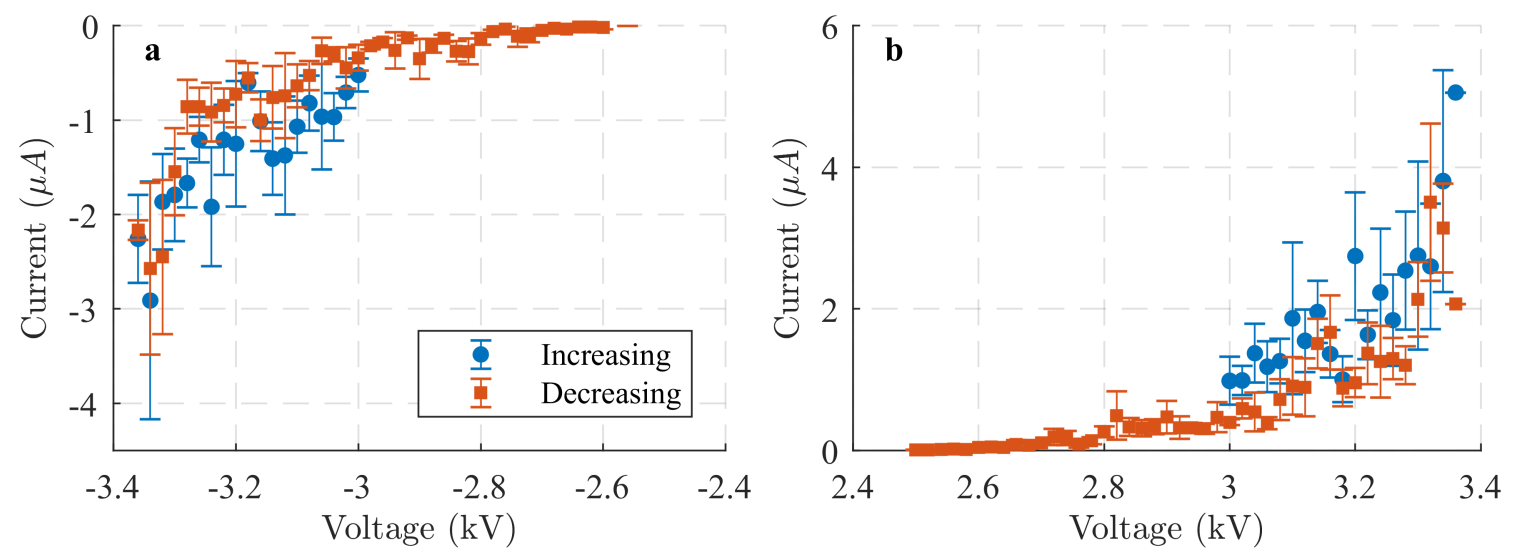

Figure 5.27: IV Traces for ILFF electrospray source operating in emission 'mode-1.' Increasing and decreasing traces are presented with the negative polarity in (a) and positive polarity in (b). (95\% CI shown).

During 'mode-2', shown in Figure 5.28, change in the extraction potential resulted in a considerable change in the emission current, relative to 'mode-1.' The emission current would then decay over a period of 30 to 90 minutes. Twice during the presented telemetry, the source self-extinguished at about 170 and 230 minutes. 


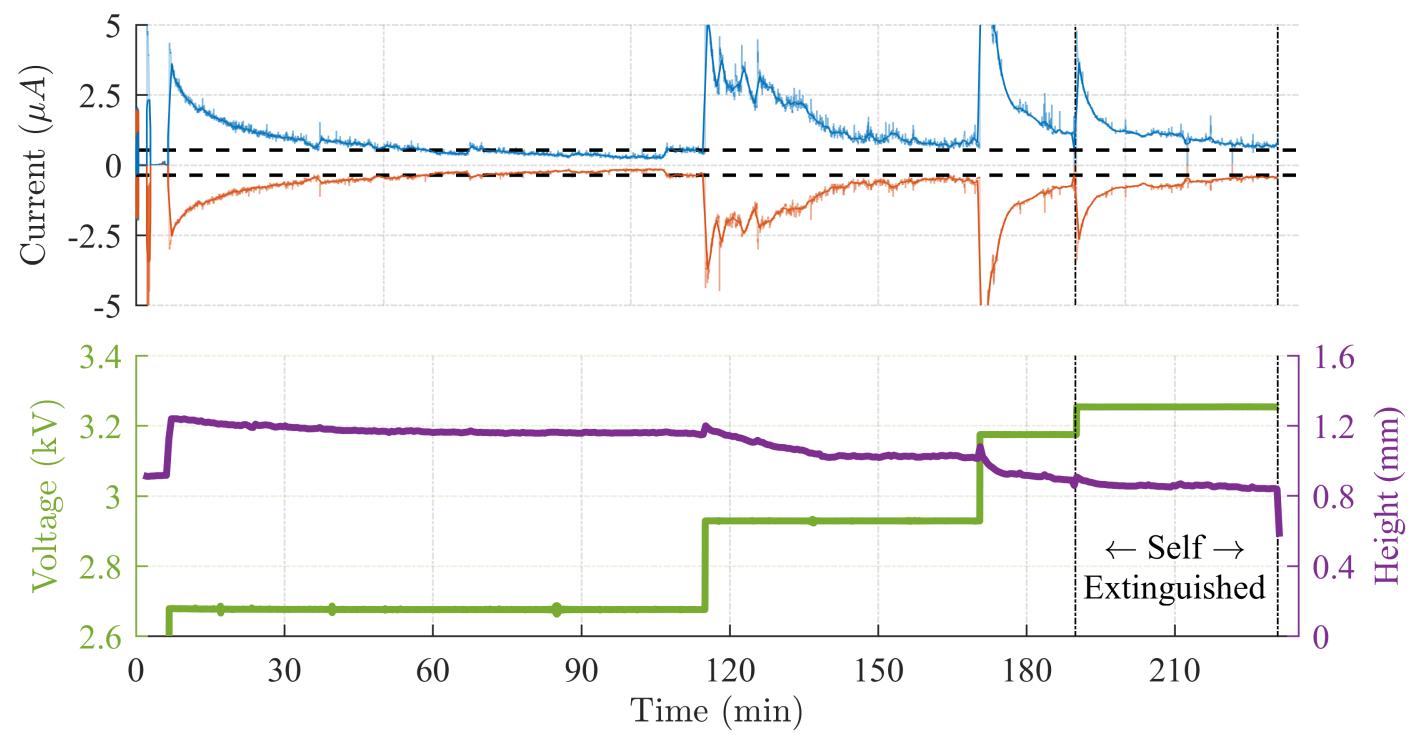

Figure 5.28: Current response to step changes in extraction potential in emission mode-2. Baseline currents of 0.53 and $-0.37 \mu \mathrm{A}$. (30 second moving average is overlayed)

In Figure 5.28, it can be observed that the emission current decays after an increase in extraction potential to a baseline emission current. This response is suggestive of two interesting phenomena. These are (1) a natural or preferred flow-rate may exist demonstrated by the emission current returning to a baseline value, and (2) the time constant of this process is rather slow (10s of minutes). It is unlikely that the source is returning to a minimum flow rate since further decrease in extraction voltage would result in a reduction in current - demonstrated in Figure 5.27. The fundamental mechanism controlling this behavior remains unknown.

Occasionally, multiple emission sites were present at the apex of the fluid meniscus. This a phenomenon, first observed by Meyer, ${ }^{30}$ was not observed at any point in the results previously in this chapter. Multiple emissions sites were only observable at relatively large increases in extraction potential, typically greater than $26 \%$ above the startup. The fluid apex is shown at multiple extraction potentials in Figure 5.29 with the corresponding telemetry in Figure 5.30. Meyer hypothesized that multiple emission sites may result from either a high volumetric flow rate or an instability resulting from the intense electric field. 


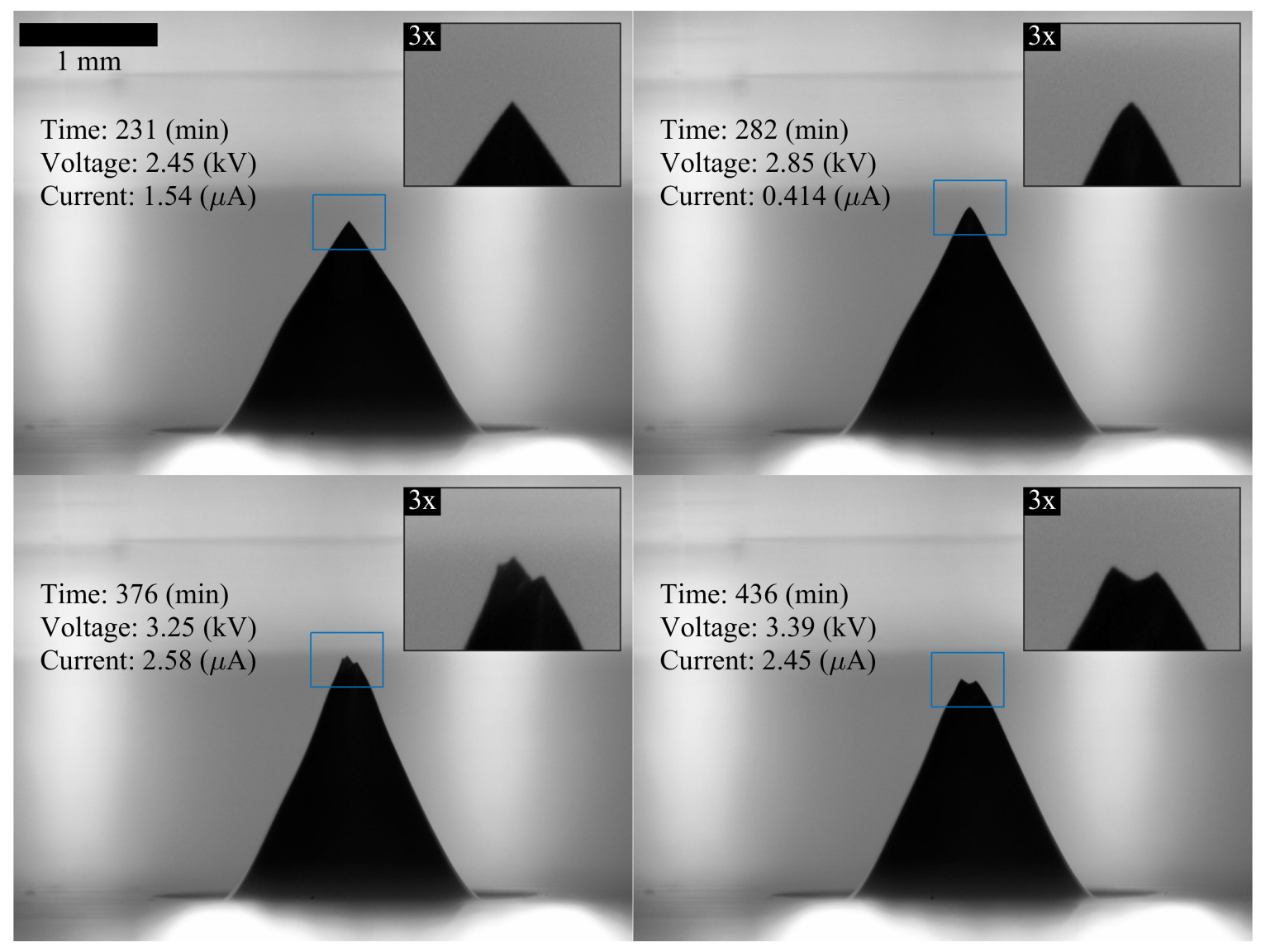

Figure 5.29: Emitter apex at high electric fields. Emitter was stable in 'mode-1' with an extraction potential of $2.45 \mathrm{kV}$. The extraction potential was gradually increased to $3.4 \mathrm{kV}$. At extraction potentials much greater than the nominal spray voltage, multiple apexes were present.

The formation of the secondary emission sites was difficult to capture with time-lapse imagery yet could easily be observed in real-time. The formation process was observed as follows. First, a very small secondary emission site materializes near the apex fluid interface. The secondary emission sites usually remain small and would self-extinguish however, would occasionally continue to grow in volume rivaling the original emission site in size. The perturbation present during the initialization process is highlighted in Figure 5.31. Finally, the multiple peaks would remain for 5 to 10 minutes before disappearing. At any time during this process, a reduction in extraction potential would result in restoration of a single emission site. 


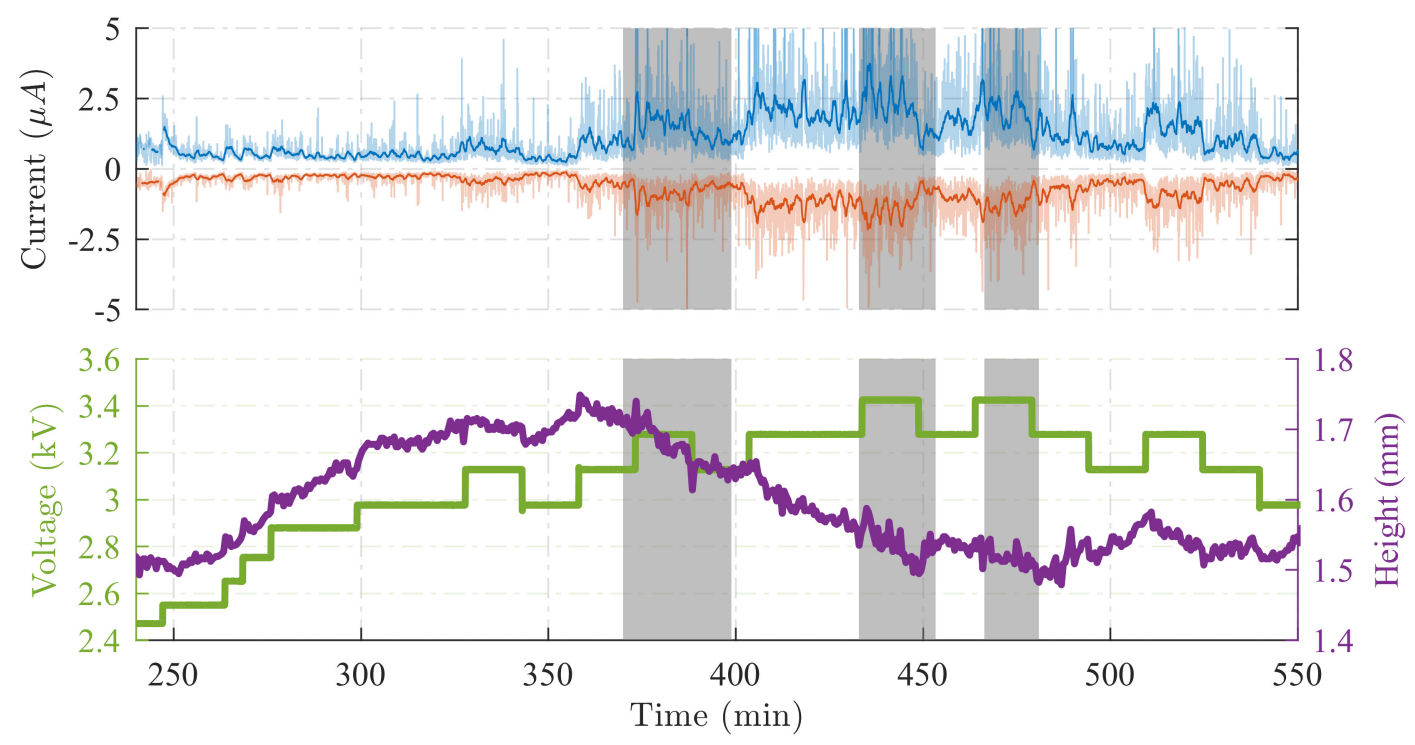

Figure 5.30: Telemetry for high extraction potential emission test during which multiple emission sites were observed. Shaded areas indicate regions where multiple peaks were observed. Between the shaded regions a single peak was observed which was very unstable and would shift frequently.

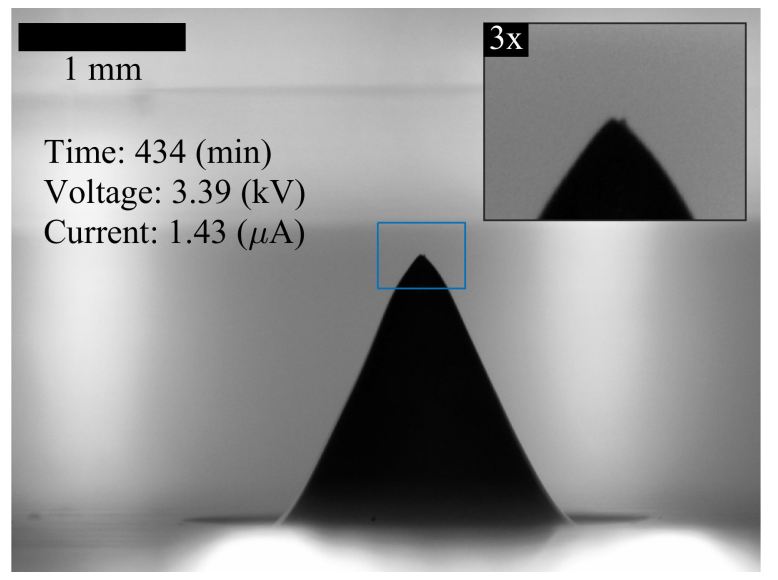

Figure 5.31: Fluid apex during the creation of a secondary emission site.

The formation of secondary emission sites when the extraction voltage is high relative to the onset voltage lends credence to the second hypothesis presented by Meyer. Meyer's hypothesis proposed that additional emission sites form as a result of an instability instigated by the higher than necessary electric field. ${ }^{30}$

During periods of intense electric field dendrite like structures were also observed to form on the fluid meniscus. These structures, shown in Figure 5.32, formed and dissipated very rapidly and lasted for a few seconds. The formation of these structures were only 
observed when the extraction potential was considerably higher than necessary to achieve emission and were accompanied by high emission currents. Similar structures were observed on a much smaller scale by Lenguito et al. ${ }^{97}$ on a $10 \mu \mathrm{m}$ ID capillary emitter post emission under "extreme electric fields." While observing electrospray with the aid of an electron microscope, Terhune et al. observed similar dendrite like structures which were attributed to fluid damage from the high energy electron beam. Terhune suggested that high energy secondary electron emission could damage the fluid. ${ }^{104}$ These secondary electrons would result when high energy electrospray ion or droplets impact downstream surfaces, freeing electrons which would be attracted back towards the emission site.

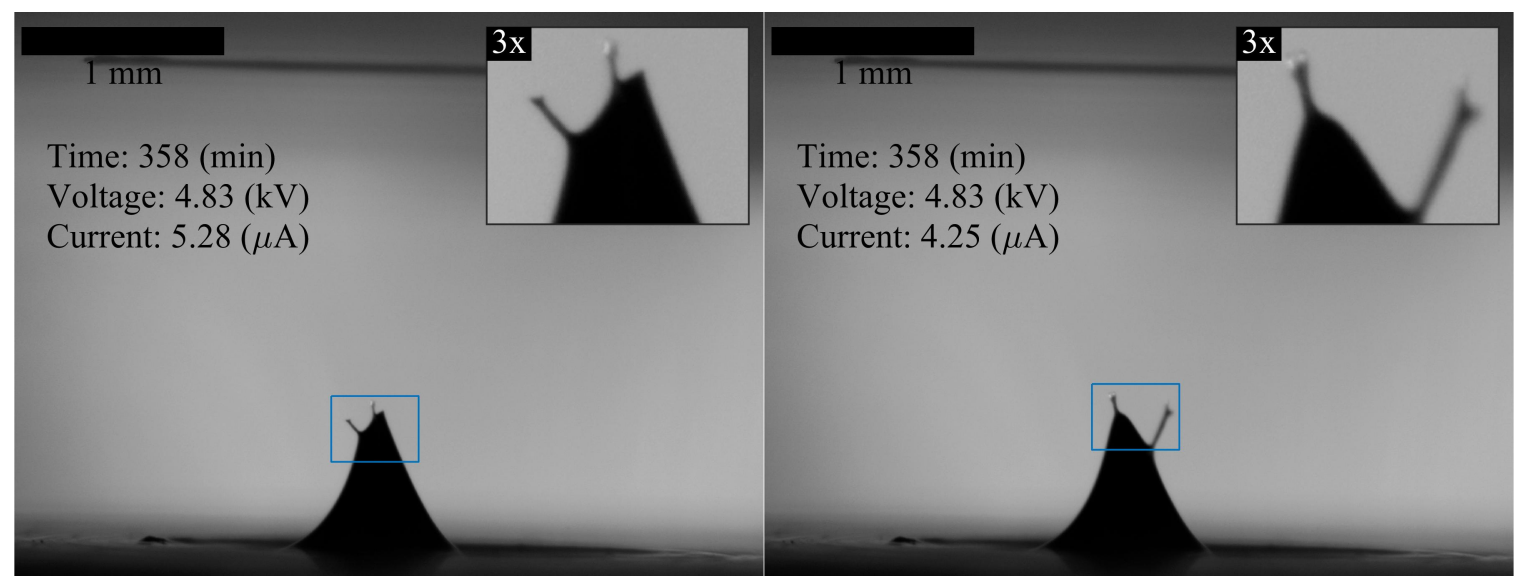

Figure 5.32: Threadlike solid structures forming on fluid meniscus during periods of extreme electric field. The image on the left was taken 7 seconds prior to the image on the right - owing to the rapid formation and destruction of the structures.

The structures observed by both Lenguito and Terhune remained after the electric field used to achieve electrospray emission was no longer present. The short-lived structures observed during this work is potentially due to the large scale of the threadlike structure, which is $2-3$ orders of magnitude larger than those observed by the mentioned authors.

\subsection{Conclusions}

The work presented in this chapter analyzed the emission behavior of an ionic liquid ferrofluid electrospray source emitting from an electrically stressed normal-field instability. The most significant finding resulting from this work is that sources of this design can emit in a stable manner with flows of around $15 \mathrm{pL} / \mathrm{s}$ for long durations. 
Emission tests demonstrated sustained emission spans of more than 15 hours - only being limited by the depletion of the emitter volume. Derived propulsion performance parameters yield a specific impulse of $1385 \mathrm{~s}$ and a thrust of $0.380 \mu \mathrm{N}$ per emitter. The low observed flow rates suggest that a large unidentified hydraulic impedance resists flow to the emission site of the passively fed electrospray source. It was theorized that nanoparticle induced flow impedance caused by bottlenecking at the emission site may provide the observed resistance.

Other experiments presented in this chapter found that the apex radius of the fluid under only magnetic stresses had a significant influence on emission dynamics. A smaller apex radius was shown to have lower flow rates and higher emission stability. It was found that the way the fluid was delivered to the fluid reservoir influenced the apex radius. 



\section{Chapter 6 Angular Emission Profile of an ILFF Electrospray Operating via the Normal-Field Instability}

Beam spreading reduces thrust efficiency for an electrospray propulsion device since off-axis particles carry non-thrust-producing momentum components. Additionally, understanding the beam divergence is critical for proper sizing of the extraction electrode orifice diameter to minimize intercepted current.
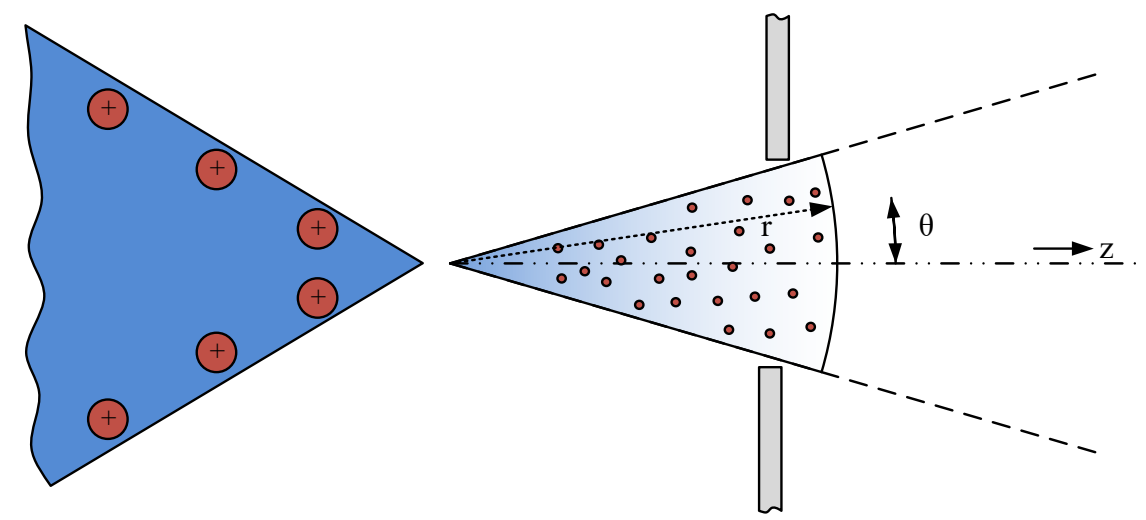

Figure 6.1: Diagram of divergence of an electrospray beam.

For simplicity, consider an electrospray source, shown in Figure 6.1, where all emitted particles have the same mass-to-charge ratio and are emitted at the potential $V_{0}$. The emitted particles are non-collinear and can be described by an angular current density distribution $\rho_{I}(\theta)$ measured at location $R_{0}$. The resulting thrust provided by such an electrospray source becomes: 


$$
\vec{T}_{\text {ideal }}=2 \pi R_{0}\left(\frac{m}{q}\right) \int_{-\theta_{\max }}^{\theta_{\max }} \frac{\rho_{I}(\theta)}{R_{0}} \vec{v}_{e}(\theta) d \theta
$$

If axial symmetry exists in $\rho_{I}(\theta)$ and it is assumed that there is no angular dependence in the particle energy potential $V_{0}$, Eq. (6.1) can be expanded as follows:

$$
T_{z, \text { ideal }}=4 \pi \sqrt{2 \frac{m}{q} V} \int_{-\theta_{\max }}^{\theta_{\max }} \rho_{I}(\theta) \cos \theta d \theta \quad \hat{z}
$$

When calculating the power utilization efficiency for an electrospray thruster in Section 1.3 , the angular power efficiency, $\eta_{\theta}$, was introduced. This factor, which accounts for the angular current distribution in the ion beam on the power utilization efficiency is defined as the following ratio:

$$
\eta_{\theta}=\left(\frac{\text { Thrust w/ ang. dep. }}{\text { Thrust w/o ang. dep. }}\right)^{2}
$$

When it is assumed that polydispersive efficiency, $\eta_{T}$, and the energy efficiency, $\eta_{E}$, account for variations in $\mathrm{m} / \mathrm{q}$ and $V$, these terms can be treated as constants when Eq. (6.2) is substituted in Eq. (6.3), which yields:

$$
\eta_{\theta}=\left(\frac{\int_{0}^{\theta_{\max }} \rho_{I}(\theta) \sin \theta \cos \theta d \theta}{\int_{0}^{\theta_{\max }} \rho_{I}(\theta) \sin \theta d \theta}\right)^{2}
$$

Consider the situation where current density in Eq. (6.4) is independent of $\theta$. For this case, the resulting effect of beam divergence on angular utilization efficiency for both thrust and power is presented in Figure 6.2. Analysis of this figure shows that for a $\theta_{\max }$ half angle of 
$40^{\circ}$, there exists a $12 \%$ reduction in thrust and a $22 \%$ reduction in power efficiency. As $\theta_{\max }$ increases, the reduction in thrust and power increases at an accelerated rate.

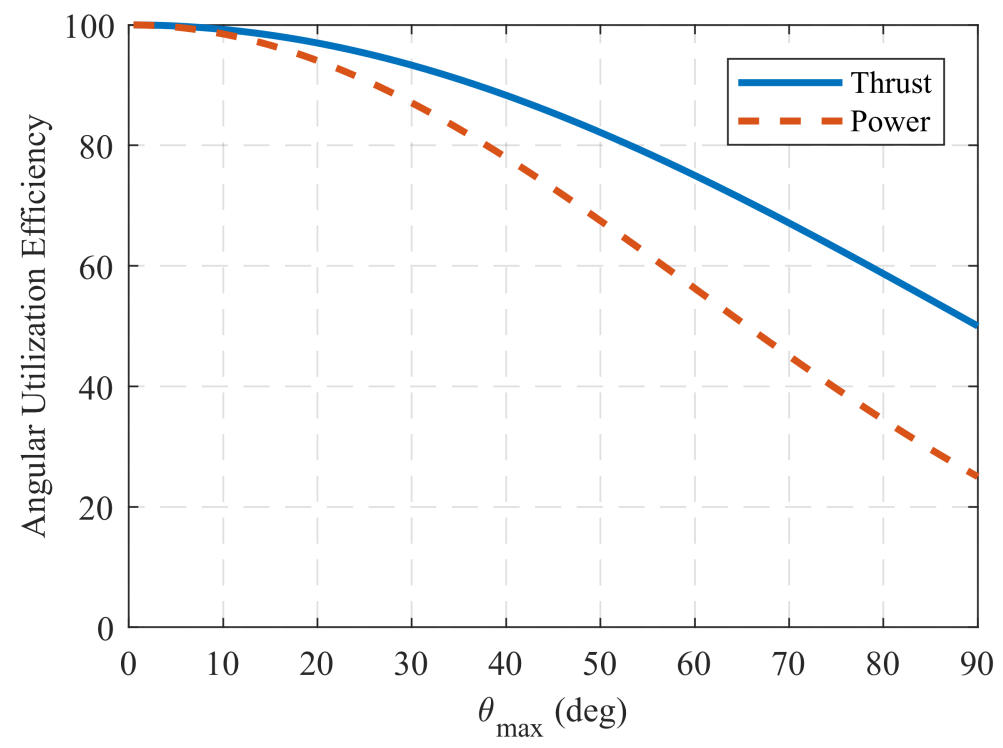

Figure 6.2: Angular and thrust utilization efficiencies assuming a constant current density, $\rho_{\mathrm{I}}$.

At this point, it becomes apparent that the overall thrust provided by an electrospray source as well as power utilization efficiency decreases with respect to a broadening of the angular current distribution. Thus, an understanding of the influential factors which control this distribution is important when developing an electrospray propulsion system.

\subsection{Goals of Study}

The primary goal of this study is to experimentally measure the angular divergence of an electrospray source and analyze factors correlated with angular divergence. The factors which will be investigated are: (1) beam polarity, (2) emission current, (3) mass flow rate, and (4) magnetic field strength. In Section 6.2 a summary of previous methods of measuring angular divergence is provided. In Section 6.3, the angular divergence profiles measured under three magnetic field conditions are presented. Finally, in Section 6.4 a 
statistical analysis is provided of influential factors on beam divergence. For this analysis, beam divergence will be presented in the form of an angular power efficiency.

\subsection{Overview of Divergence Measuring Techniques}

An initial angular divergence study using ionic liquid ferrofluids was performed in the summer of 2014 by the author. ${ }^{105}$ In summary, this approach swept a Faraday probe in a circular profile over an emission source using a stepper motor. This approach was able to achieve excellent angular resolution. However, the method was rather slow, taking about 25 seconds for a complete sweep and thus was unable to differentiate between temporal and spatial profile variations. The limitations of this previous approach motivated the work presented herein.

Multiple approaches have been employed by various authors to obtained beam divergence measurements. Chiu et. al. ${ }^{105}$ and Courtney ${ }^{24}$ et. al. both obtained angular current distribution measurements by rotating an electrospray source and extractor electrode with respect to a measurement device. Gamero-Castaño obtained beam profile measurements by moving a collector plate mounted on a motion stage over an emission source. $^{40,106}$ Spray measurements performed by Morris were obtained by a slit-shaped aperture between the extraction electrode and Faraday plate collector using a motion stage. In this method, only the spray that passed through the aperture at a given known position was measured. ${ }^{107}$ In general, these methods achieve excellent spatial resolution but poor temporal resolution.

In 2003, Lozano presented a study which measured the angular divergence of needlebased traditional electrospray emitters. ${ }^{108}$ The approach employed for this study consisted of a stack of 11 electrically-isolated concentric ring plates. Current was measured on each ring along various axial distances to obtain the beam spreading as a function of downstream position. This approach is capable of performing simultaneous measurements, allowing for time resolved measurements to be made of the intercepted current on each collector at the cost of decreased spatial resolution. 


\subsection{In Situ Measurement of Angular Current Distribution Methodology and Results}

To obtain measurements of the angular divergence of the electrospray source, the emitter was operated in the manner described in Section 5.2.5. The segmented current collector, discussed in detail in Section 5.2.3 was employed. Current measurements are reported at the midline of the annular shaped Faraday probe, yielding angular measurements at the locations specific in Table 6-1. An example of the typical telemetry showing emission current collected on all nine Faraday probes is presented in Figure 6.3.

Table 6-1: Faraday probe area and angular position. Angular position referenced to the midline of each annulus.

\begin{tabular}{|c|c|c|c|c|c|c|c|c|c|}
\hline Probe \#: & 9 & 8 & 7 & 6 & 5 & 4 & 3 & 2 & 1 \\
\hline Area $\left(\mathrm{mm}^{2}\right)$ & 6.1 & 12.4 & 19.5 & 26.6 & 33.7 & 40.8 & 47.9 & 142.1 & 176.6 \\
\hline Angle (deg) & 0 & 8.2 & 12.7 & 17.1 & 21.3 & 25.2 & 28.9 & 34.1 & 40.0 \\
\hline
\end{tabular}

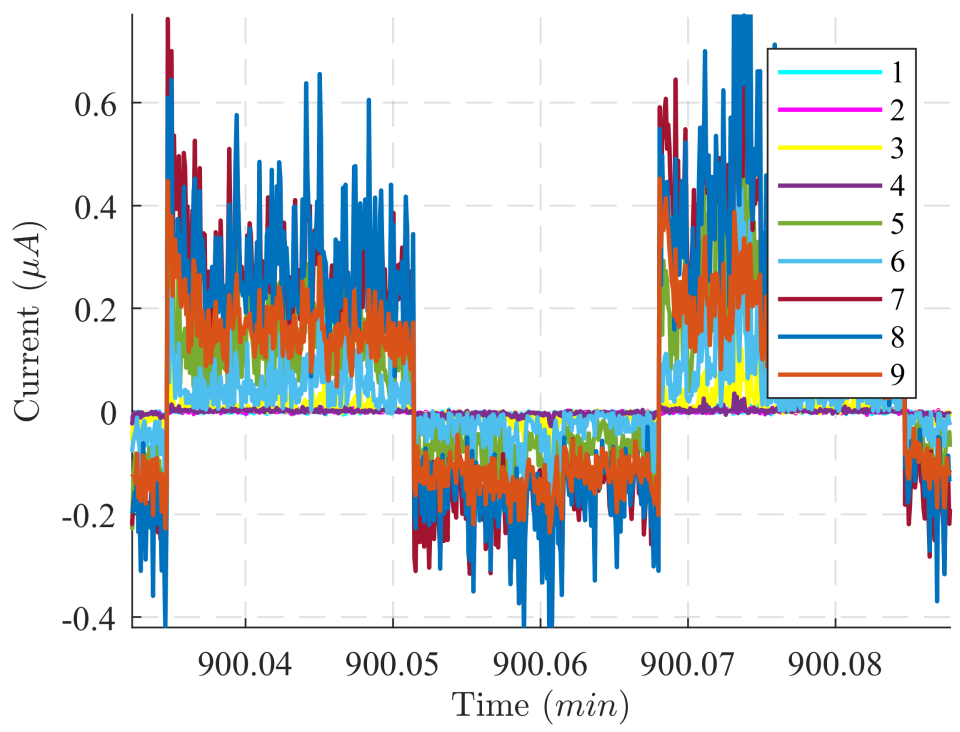

Figure 6.3: Typical Faraday trace obtained by simultaneously measuring current on all nine collectors. 
Angular plume current density measurements obtained from the source are presented at select 20-minute intervals for three different magnetic field strengths, presented in Figures 6.4 through 6.6. Averages over 1-second intervals are shown in gray and the 20minute average is shown in black with $95 \%$ confidence intervals. A comparison of the results shown in Figure 6.7.

From a purely visual observation, no discernible trend in angular divergence is noticeable. Each source, regardless of field strength, exhibited a similar distribution at comparable times within the telemetry. As emission progresses, an interesting observation can be made regarding the transformation of the emission current density distribution. After two to three hours of testing, the source was observed to have a decreasing angular density, with the highest current density on the pad located at $0^{\circ}$. As emission proceeded, a transition was observed where the highest current density was generally on the second radial Faraday plate, located at $8.16^{\circ}$. Beam measurements performed by Lozano ${ }^{108}$ had a region of increasing current density with respect to radial position. Courtney et al. ${ }^{25}$ also observed current distributions exhibiting off-center peaks while measuring the full beam profile while spraying an ionic liquid.

Findings by Gamero-Castaño ${ }^{106}$ showed an evolution in the beam profile as a function of beam current - transforming from a parabolic-like shape to a broader profile with multiple local maximums with increasing beam current. Gamero-Castaño sprayed propylene carbonate doped with an ionic liquid. The observed trend was attributed to a superposition of two droplet populations, the center distribution consisting of main droplets and the outer beam consists of satellite droplets. 

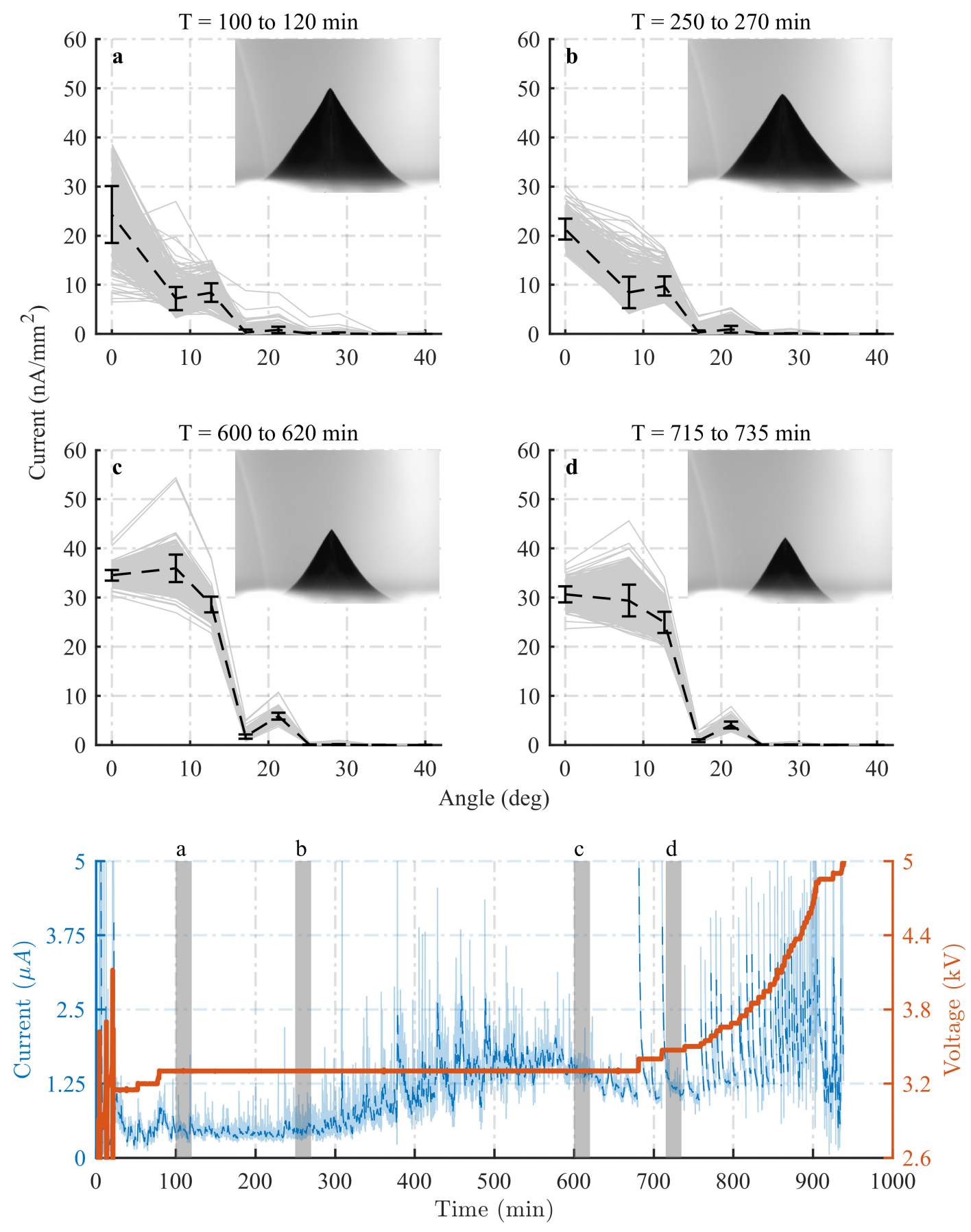

Figure 6.4: (321 G) Angular plume current distribution at select intervals. 1-minute intervals shown in gray, a 20-minute average is shown in black with a $95 \%$ confidence interval. 

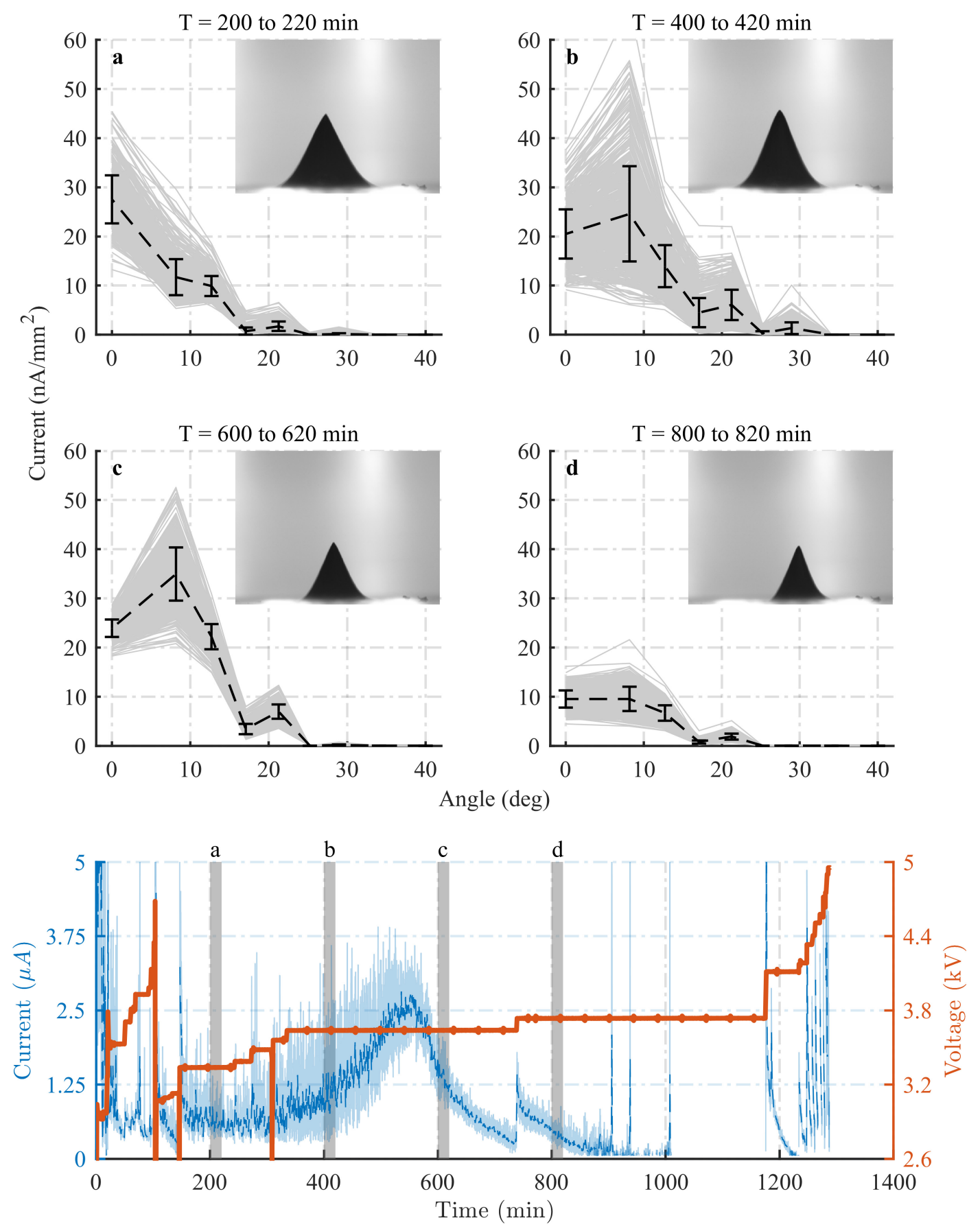

Figure 6.5: (388 G) Angular plume current distribution at select intervals. 1-minute intervals shown in gray, a 20-minute average is shown in black with a $95 \%$ confidence interval. 

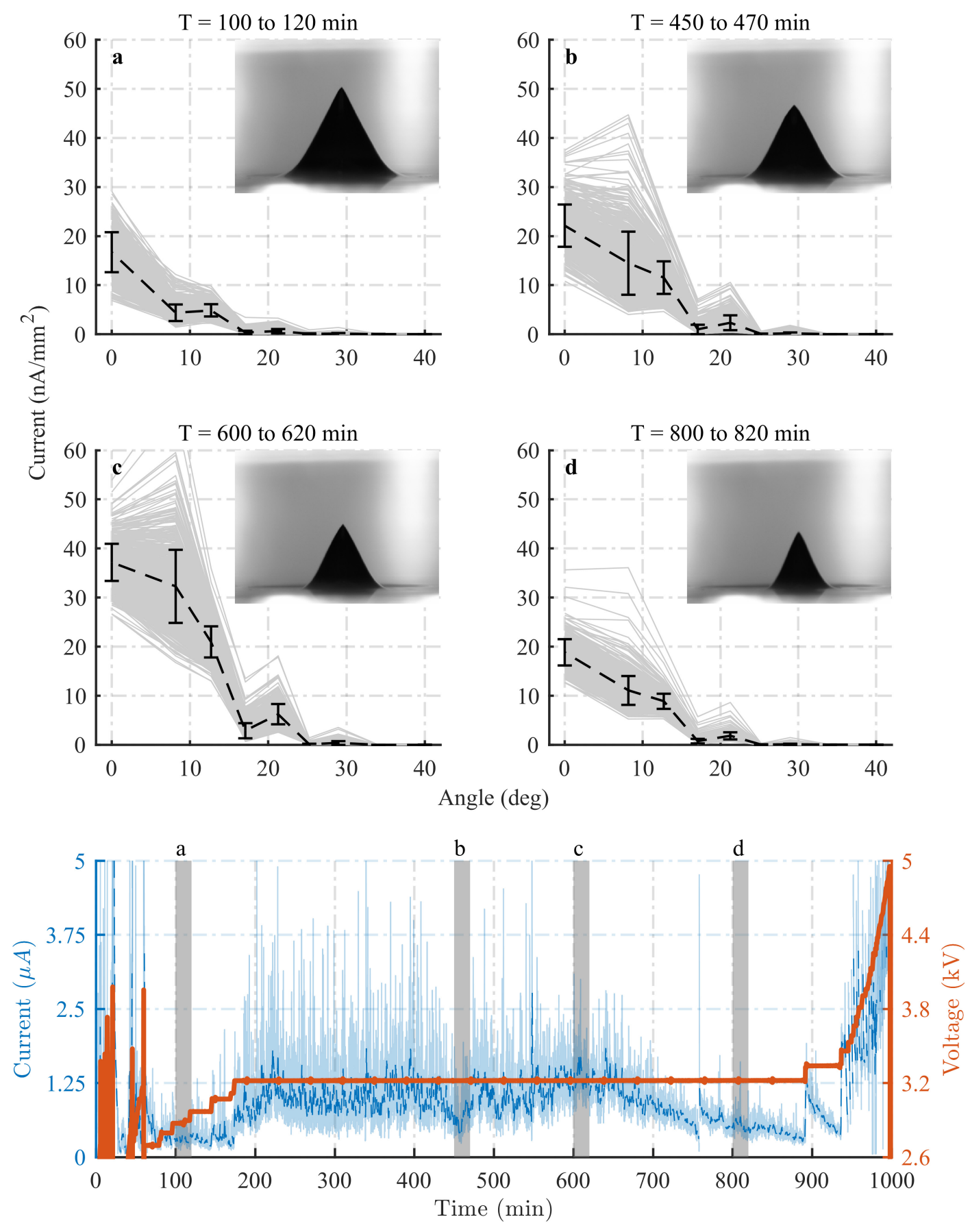

Figure 6.6: (495 G) Angular plume current distribution at select intervals. 1-minute intervals shown in gray, a 20-minute average is shown in black with a $95 \%$ confidence interval. 

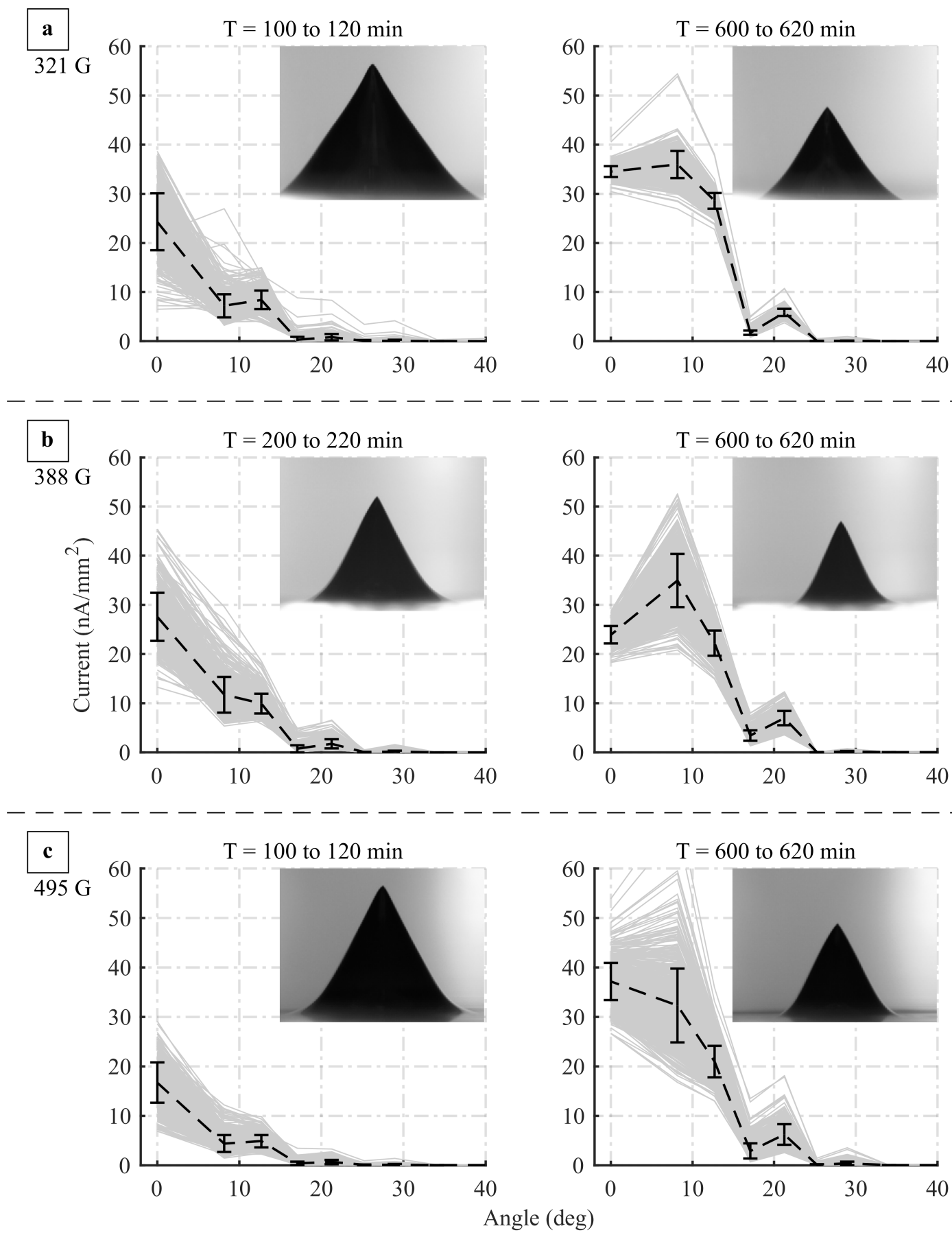

Figure 6.7: Comparison of angular plume current distribution for three magnetic field strengths (a) $321 \mathrm{G}$, (b) $388 \mathrm{G}$, and (c) 495 G. (95\% CI shown). 


\subsection{Angular Power Efficiency Factor}

The angular power efficiency factor, $\eta_{\theta}$, was calculated during the duration of the emission telemetry at regular intervals. The angular current distribution function $\rho_{I}(\theta)$ was determined by applying a Piecewise Cubic Hermite Interpolating polynomial (PCHIP) function to the area weighted Faraday probe measurements. The angular current distribution function was then integrated using Eq. (6.4) to obtain the angular power efficiency factor. A more detailed discussion of the numerical methodology for calculating the efficiency factor is presented in Appendix E. For the analyses presented in this section, the angular efficiency was calculated by averaging 1-minute spans every 10 minutes during the telemetry. When angular efficiencies are presented in graphical form, only $1 / 3^{\text {rd }}$ of the data points are shown to increase readability.

The unstable emission behavior during the startup and terminal phase of the testing resulted in several angular efficiencies that can be considered outliers. These points, numbering 3,6, and 1 out of a population of 86 for the 321,388 , and $495 \mathrm{G}$ test respectively were identified and excluded from the analysis using a median absolute deviation technique. Sample points more than three scaled median deviations from the median were determined to be outliers. The omitted sample points existed in the angular efficiency ranging from 0.4 to 0.918 .

The evolution in time of the angular efficiency for three different magnetic fields is presented in Figure 6.8, with a tabulated comparison of the mean efficiency in Table 6-2. The mean angular efficiency for the $321 \mathrm{G}$ and $495 \mathrm{G}$ datasets are comparable while the $388 \mathrm{G}$ dataset is slightly lower by about $1.1 \%$. The $388 \mathrm{G}$ dataset, which is the outlier of the two, experienced a rise and fall in emission current between the 300 and 700 minutes in the telemetry, with a peak current occurring around 550 minutes (see Figure 6.5). A corresponding trend appearing as a decreasing angular efficiency during this same period, can be observed in Figure 6.8. The higher emission current and decreased efficiency corresponding with this test case is suggestive of a correlation between emission current, 
plume divergence, and net efficiency, a relation that will be investigated further in Section 6.4.2.

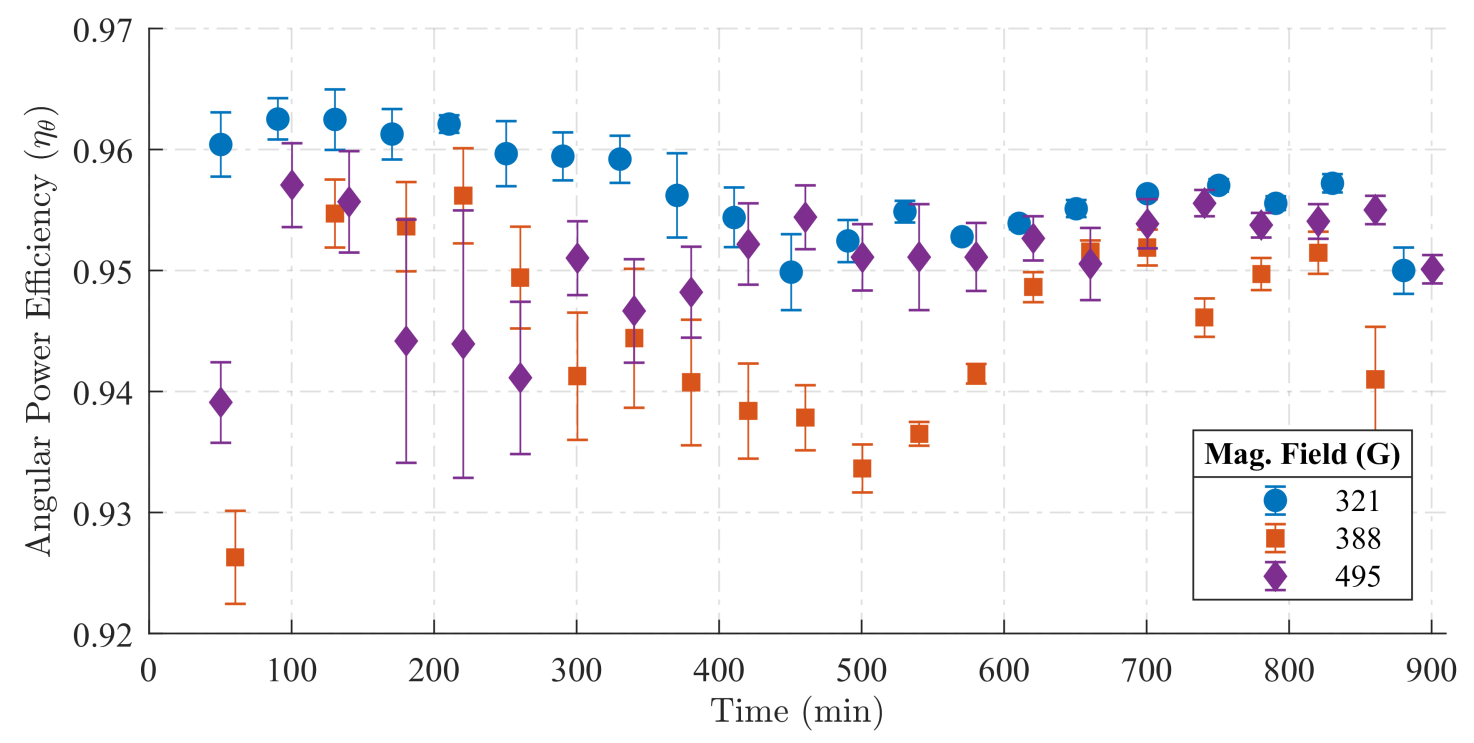

Figure 6.8: Angular power efficiency during emission for three magnetic field conditions.

Table 6-2: Tabulated comparison of angular power efficiency. Only angular efficiencies after 200 minutes were included in the averaging since prior to that time, not all sample sets were in the constant voltage operation mode.

\begin{tabular}{cc}
\hline $\begin{array}{c}\text { Mag. Field } \\
(\mathrm{G})\end{array}$ & Mean $\eta_{\theta}$ \\
\hline \hline 321 & $0.9463 \pm 0.0065$ \\
388 & $0.9365 \pm 0.0068$ \\
495 & $0.9470 \pm 0.0047$ \\
\hline
\end{tabular}

The small population size with respect to magnetic field did not permit any statistical analysis to be performed regarding the influence of the magnetic field strength to emission behavior. 


\subsubsection{Dependence on Beam Polarity}

As noted in Section 5.3, more current was emitted in the positive polarity relative to the negative polarity. However, when scaled with respect to their maximum emission densities, the profile of the two have a similar form, as indicated by Figures 6.9 and 6.10 .

A quantitative analysis of the polarity influence on the plume divergence can be obtained from angular propulsion efficiency factor for positive and negative emission cycles. A comparison between the polarities for all three datasets is presented graphically Figure 6.11 where a systematically lower efficiency can be observed for the negative polarity for all datasets. Using a Welch's t-test, it was confirmed positive polarity had a higher angular efficiency than the negative polarity with a 95\% significance. Results from this statistical analysis are shown in Table 6-3. In table, the p-value measures the probability that there is no statistical difference between the populations. A p-value of less than 0.05 is typically used to assume statistically strong evidence for the rejection of the null hypothesis.
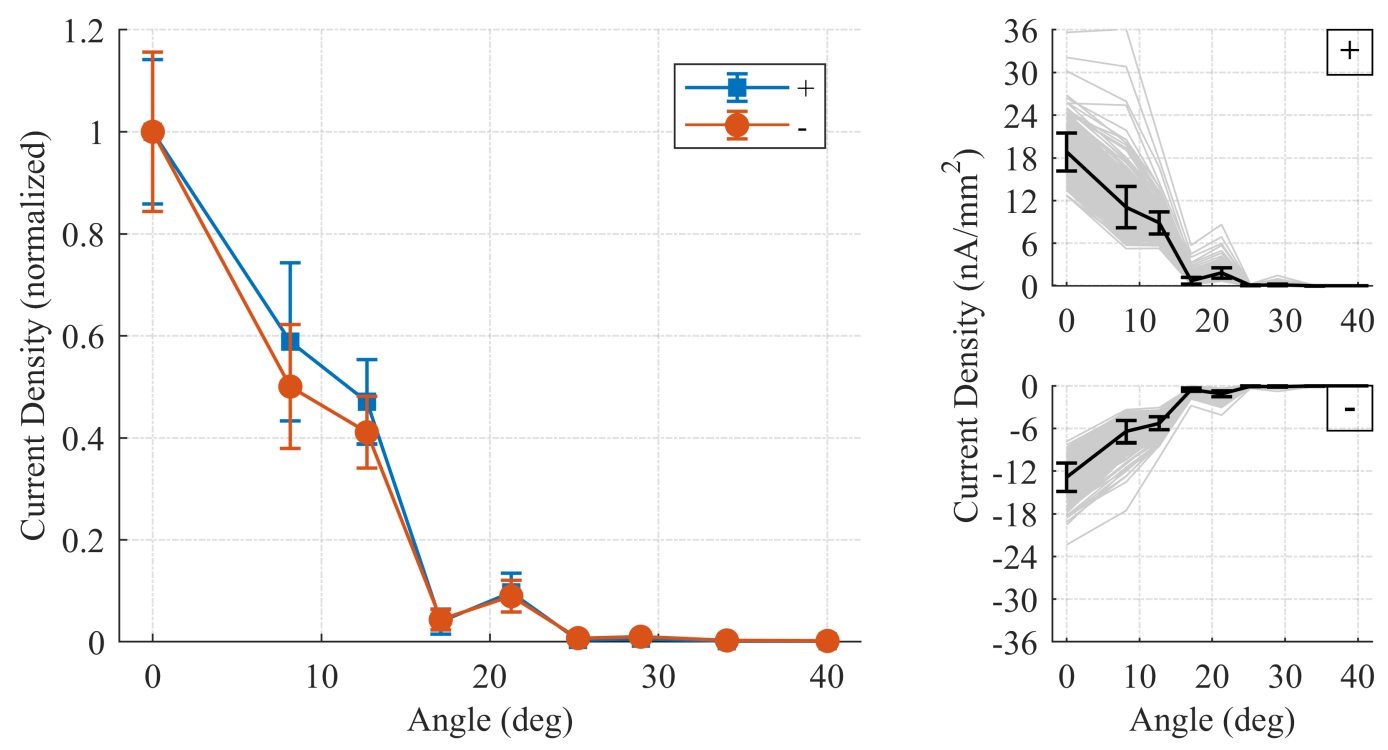

Figure 6.9: Normalized current density for positive and negative emission modes. (B = $495 \mathrm{G}$ Averaged between 100-120 minutes into telemetry). 

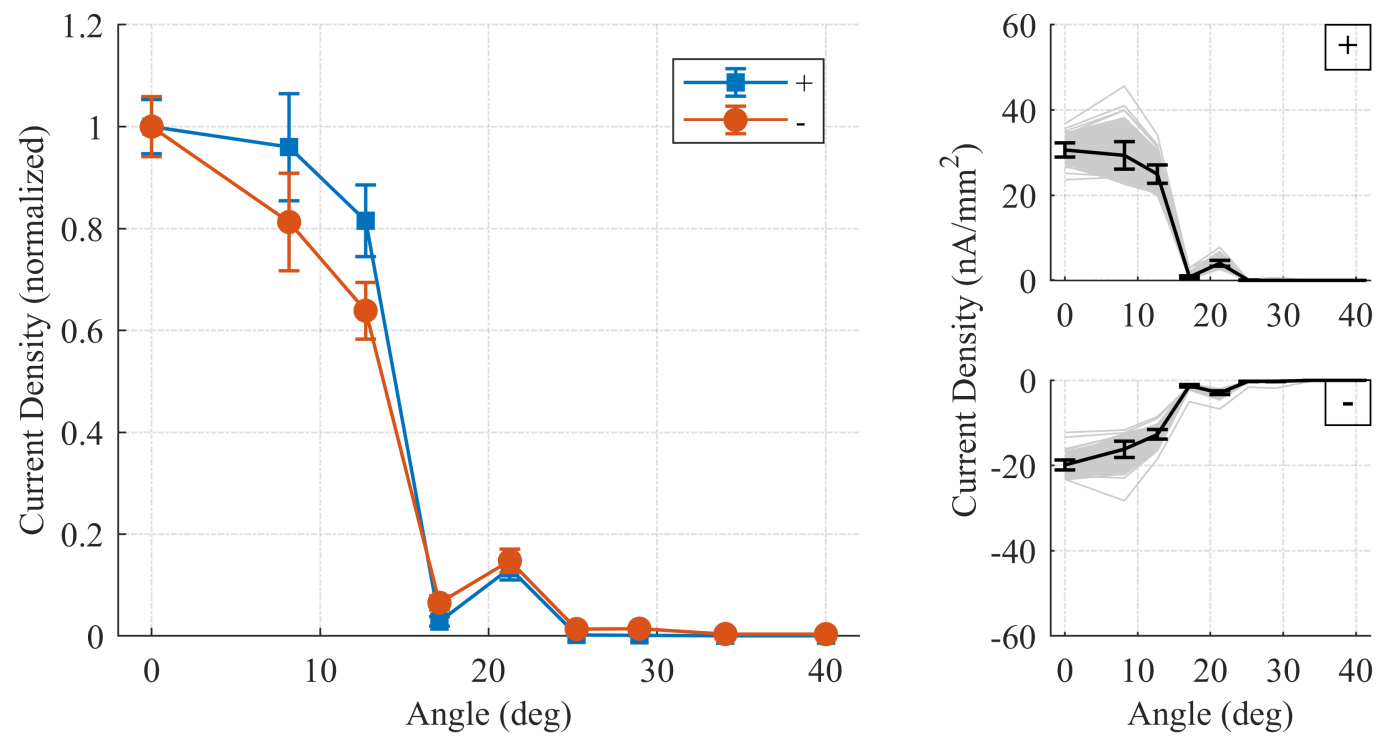

Figure 6.10: Normalized current density for positive and negative emission modes. (B $=321 \mathrm{G}$ Averaged between 600-620 minutes into telemetry).

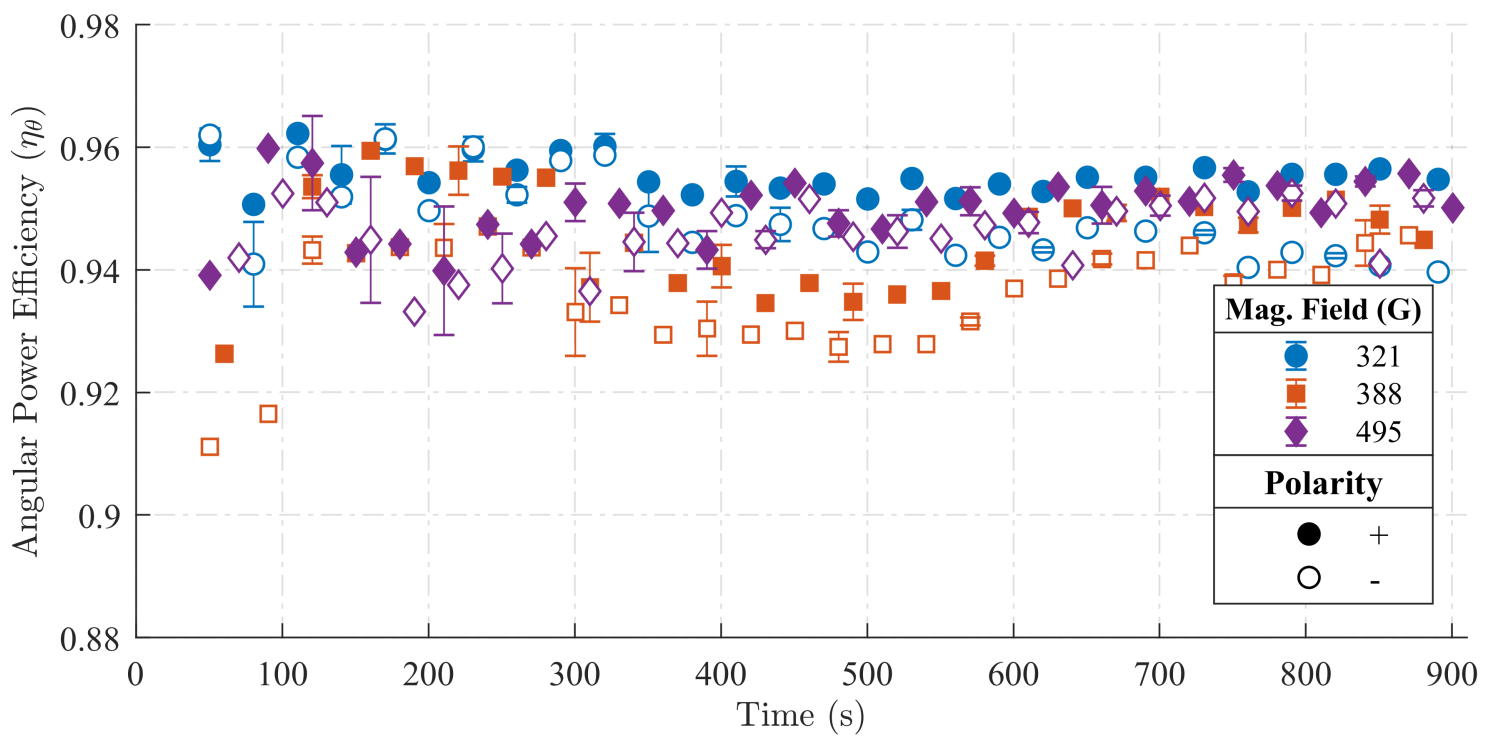

Figure 6.11: Angular power efficiency for positive and negative emission current polarity for three magnetic fields. Sparse error bars shown with $95 \%$ CI. 
Table 6-3: Comparison in angular efficiency between positive and negative emission polarities. P-value calculated using a two-sample t-test.

\begin{tabular}{ccccc}
\hline Mag. Field & \multicolumn{2}{c}{ Pop. $(n)$} & Pct. Diff. & P-Value \\
$(\mathbf{G})$ & $(+)$ & $(-)$ & & \\
\hline 321 & 83 & 83 & 0.75 & $3.10 \times 10^{-14}$ \\
388 & 80 & 82 & 1.02 & $1.59 \times 10^{-11}$ \\
495 & 85 & 84 & 0.48 & $1.06 \times 10^{-7}$ \\
\hline
\end{tabular}

A difference in beam divergence of positive and negative polarity can be reasonably expected under the well-accepted premise that beam composition depends on polarity and that the composition of the beam has a significant influence on the divergence of the beam. Chiu et al. performed a very comprehensive study using an externally wetted electrospray source emitting EMIM-NTf2, the same ionic liquid which serves as a carrier fluid for the ferrofluid studied herein. In this work, emission current and mass flow rate were measured with respect to emitter angle, which yielded distributions which are dependent on the current polarity. Using a quadrupole mass filter, their work also demonstrated that the beam composition dependent strongly on polarity as well as angle. In a comprehensive analysis of the 480 emitter iEPS thruster, Krejci et al. observed a much larger polarity dependence in angular efficiency of 0.801 and 0.828 for the positive and negative polarities, respectively. ${ }^{1}$

\subsubsection{Dependence on Emission Current and Mass Flow Rate}

While researching capillary needle emitters, Gamero-Castaño observed a broadening of the emission plume with an increase in emission current when emitting both EMIM$\mathrm{NTf}^{40}$ as well as with propylene carbonate doped with an ionic liquid. ${ }^{106}$ This broadening observed was attributed to an increase in satellite droplet formation. Gamero-Castaño also observed an increased production of higher mass-to-charge droplets at propellant flow rate. ${ }^{40}$ Since particle of different mass-to-charge ratios respond differently to acceleration 
forces in the apex region, charge-to-mass ratios of the emitted species are accepted to be an influential factor in beam divergence.

To identify the influence of emission current and mass flow rate on angular efficiency a correlation analysis was performed. The relationship between emission current and efficiency, mass flow rate and efficiency, and mass flow rate and current can be observed in Figures 6.12 through 6.14, respectively. The mass flow rate for the subsequent analysis is the net flow rate of the emitter, while the current and efficiency are reported for a single polarity. It is fair to assume that the flow rate in the positive polarity is linearly proportional to the net mass flow rate, thus the correlations coefficients are unaffected by this assumption.

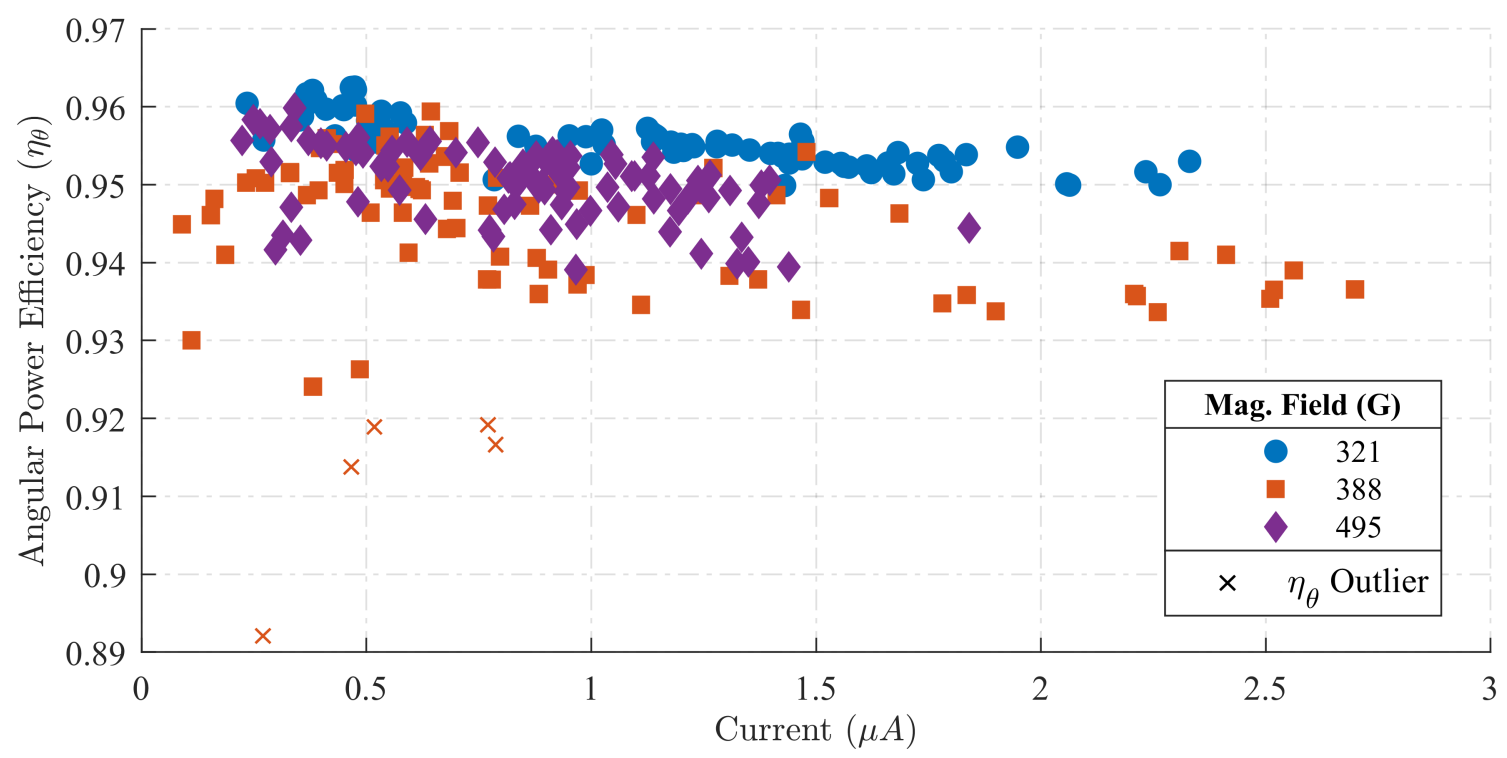

Figure 6.12: Angular efficiency and emission current correlation. 


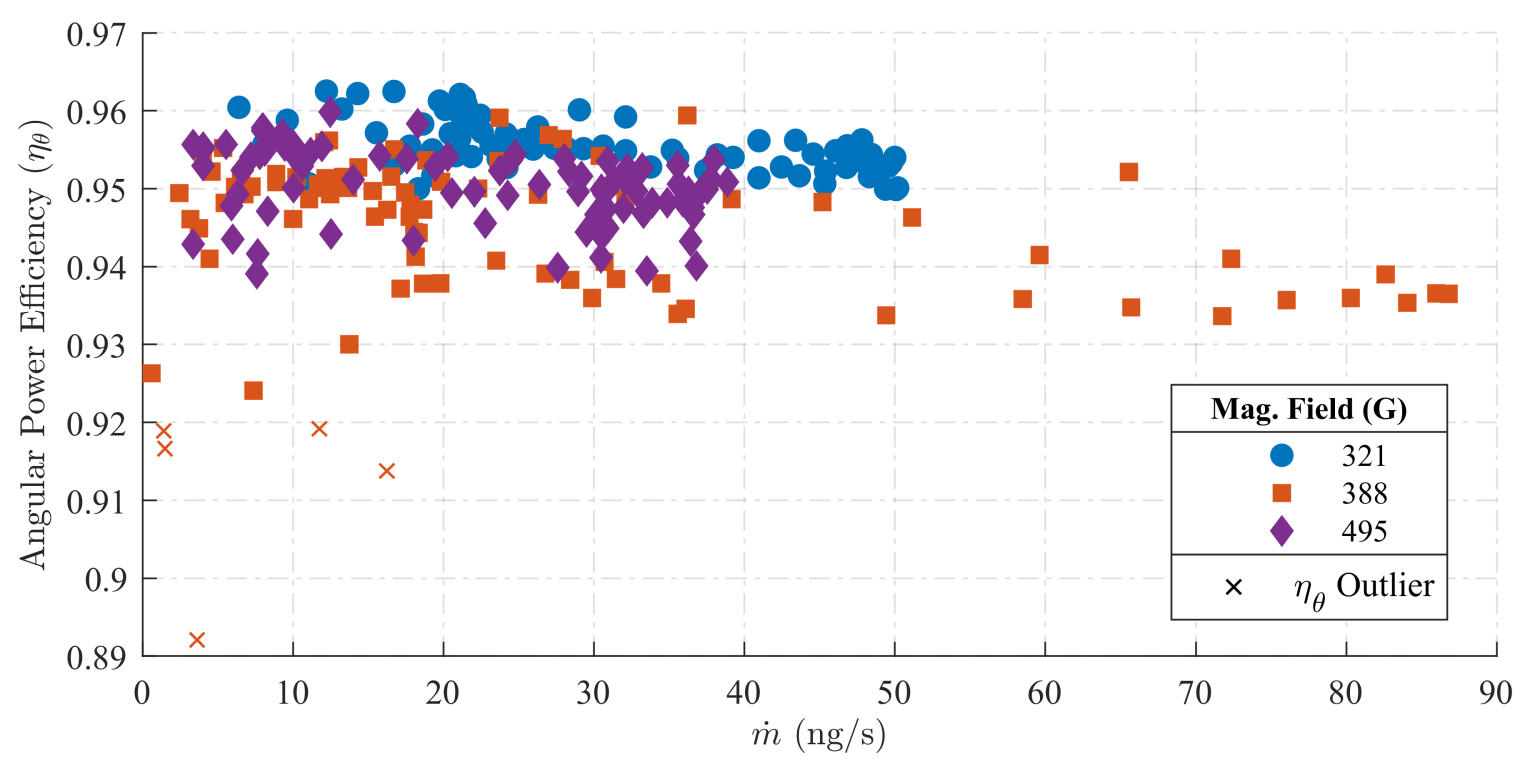

Figure 6.13: Angular efficiency and mass flow rate correlation.

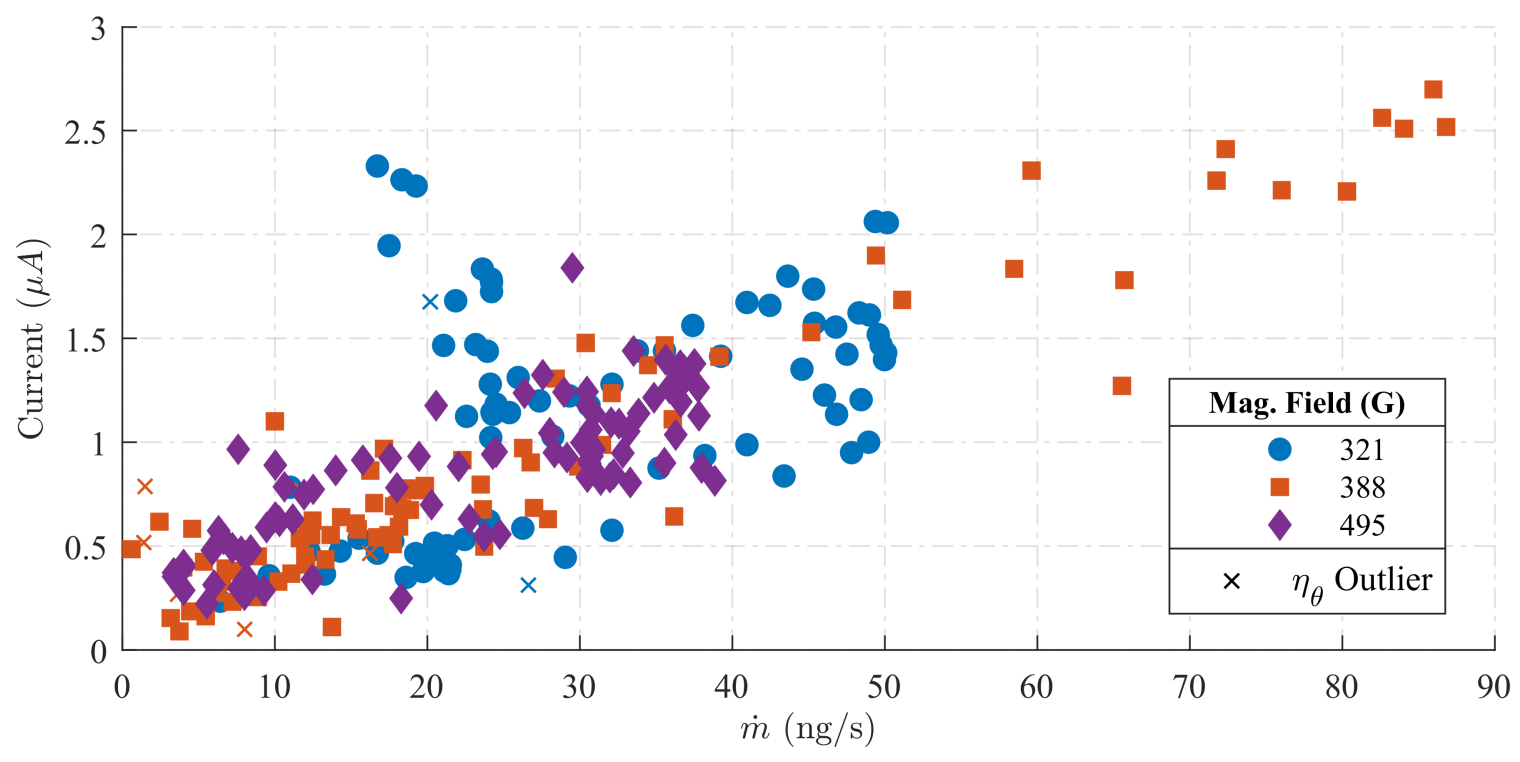

Figure 6.14: Emission current and mass flow rate correlation.

A tabulated comparison of the correlations using the Spearman technique is presented in Table 6-4. Analysis using both the Pearson and Kendall method were also conducted, which yielded similar results. Correlation tables for these methods can be found in Appendix E. The p-value measures the probability that the null hypothesis is true, which is that there is zero correlation between the two variables of consideration. This analysis yields a strong positive correlation between emission current and mass flow rate, a strong 
negative correlation between emission current and angular efficiency, and a moderately negative correlation between mass flow-rate and angular efficiency. These correlations are accompanied by a significantly small p-values, ranging from 0 to $10^{-4}$, yielding excellent certainty in the rejection of the null hypothesis.

Table 6-4: Tabulated correlation coefficients for mass flow rate, emission current, and angular power efficiency. Values correlated using the Spearman correlation technique.

\begin{tabular}{|c|c|c|c|c|}
\hline \multicolumn{3}{|c|}{$\begin{array}{l}\text { B Field } \\
\text { (G) }\end{array}$} & $\eta_{\theta}$ & $I$ \\
\hline \multirow{6}{*}{$\dot{m}$} & \multirow{2}{*}{321} & $\overline{\mathrm{R}}$ & -0.557 & 0.507 \\
\hline & & $\mathrm{P}$ & $8.07 \times 10^{-8}$ & $1.42 \times 10^{-6}$ \\
\hline & \multirow{2}{*}{388} & $\mathrm{R}$ & -0.383 & 0.893 \\
\hline & & $\mathrm{P}$ & $4.90 \times 10^{-4}$ & 0 \\
\hline & \multirow{2}{*}{495} & $\mathrm{R}$ & -0.355 & 0.774 \\
\hline & & $\mathrm{P}$ & $9.31 \times 10^{-4}$ & 0 \\
\hline \multirow{6}{*}{$I$} & \multirow{2}{*}{321} & $\mathrm{R}$ & -0.829 & \multirow{2}{*}{$\mathrm{n}=83$} \\
\hline & & $\mathrm{P}$ & 0 & \\
\hline & \multirow{2}{*}{388} & $\mathrm{R}$ & -0.457 & \multirow{2}{*}{$\mathrm{n}=80$} \\
\hline & & $\mathrm{P}$ & $2.47 \times 10^{-5}$ & \\
\hline & \multirow{2}{*}{495} & $\mathrm{R}$ & -0.506 & \multirow{2}{*}{$\mathrm{n}=85$} \\
\hline & & $\mathrm{P}$ & $1.15 \times 10^{-6}$ & \\
\hline
\end{tabular}

The high negative correlation between efficiency and emission current is consistent with the previously cited work by Gamero-Castaño while the influence of mass flow rate and efficiency is supported by Miller et al. ${ }^{109}$ A relationship between mass flow rate and emission current is well documented for capillary needle emitters. Fernández de la Mora and Loscertales ${ }^{53}$ presented a relation for a "highly conductive" fluids which relates mass flow rate and current via the following relationship:

$$
I \sim Q^{S}
$$

where $S$ was found to be approximately 0.5 over a broad range of fluid conductivities. Recognizing that $Q$ and $\dot{m}$ can be interchanged in this relation without impacting the 
coefficient $S$, a similar relation can be established for this source. A fit of Eq. (6.5) to the current mass flow rate relationship is presented below in Figure 6.15 while the tabulated fit with a 95\% confidence interval is presented in Table 6-5.

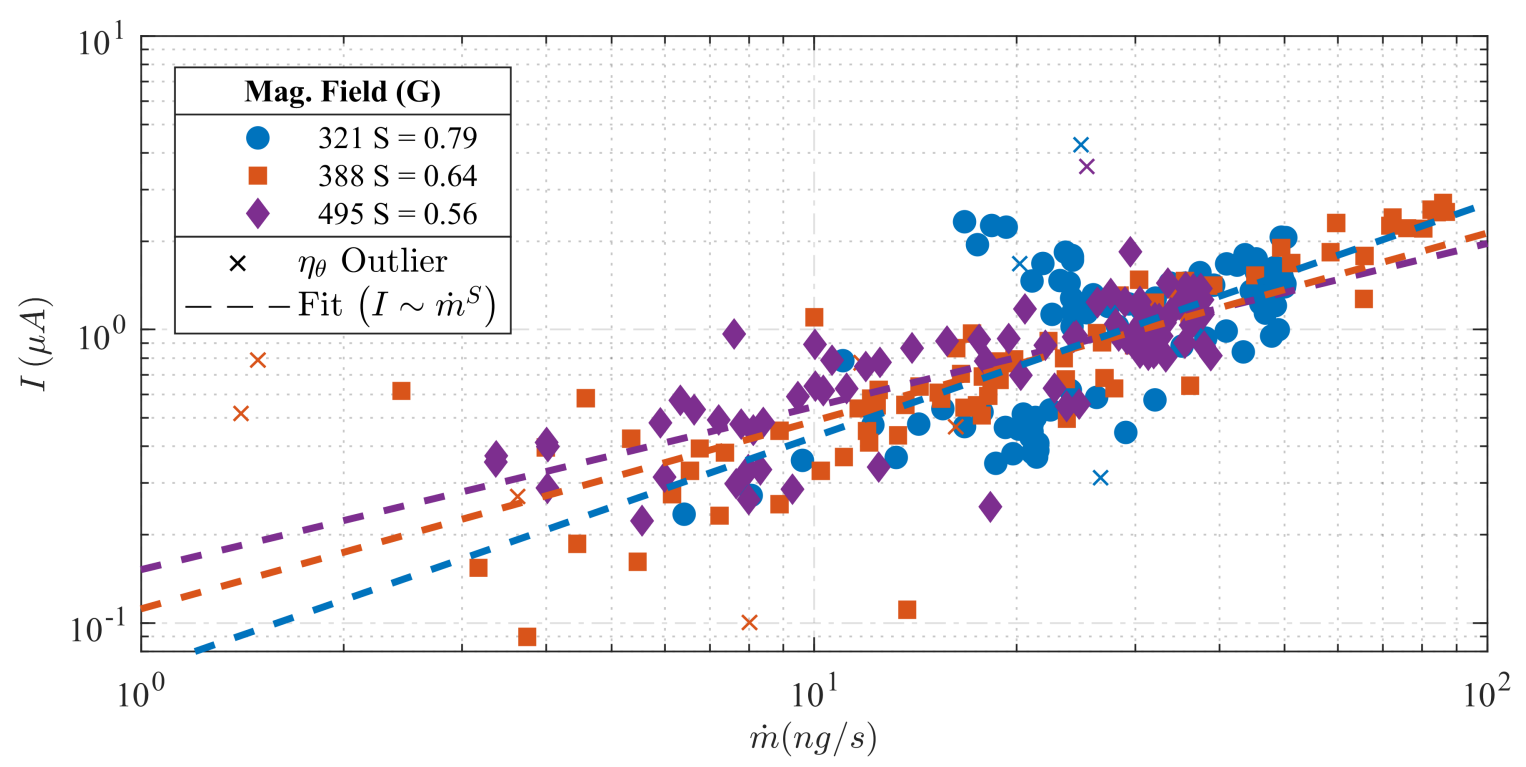

Figure 6.15: Log-Log plot of emission current vs mass flow rate. $S$ is the regression coefficient per Eq. (6.5).

Table 6-5: Tabulated scaling of the emission current and mass flow rate regression coefficient.

\begin{tabular}{cccc}
\hline $\begin{array}{c}\text { Mag. Field } \\
\text { (G) }\end{array}$ & Pop. $(n)$ & $(S)$ & $\mathbf{9 5 \%}$ CI \\
\hline 321 & 83 & 0.795 & {$[0.565,1.02]$} \\
388 & 80 & 0.640 & {$[0.536,0.744]$} \\
495 & 85 & 0.556 & {$[0.467,0.645]$}
\end{tabular}

The regression coefficient observed for the normal-field electrospray source, averaging to 0.664 , differs from the 0.5 coefficient observed by Fernández de la Mora and Loscertales for highly-conductive liquids. However, da la Mora notes that the previously mentioned scaling law is applicable for fluids with conductivities between $10^{-4}$ and $1 \mathrm{~S} / \mathrm{m}$. For fluid 
conductivities around $1 \mathrm{~S} / \mathrm{m}$, ion evaporation results at the fluid meniscus and the scaling law breaks down. ${ }^{38}$ The conductivity for the ferrofluid emitted in this study was measured to be $0.63 \mathrm{~S} / \mathrm{m}$ (Table 4-1, Pg. 36), within the range at which the 0.5 model breaks down. The difference between 0.664 coefficient and the 0.5 coefficient presented by de la Mora is most accurately explained by the presence of ion evaporation from the apex of the ferrofluid meniscus. Ion emission would result in an increased emission current with a negligible mass flow rate increase - resulting in a greater regression coefficient $S$. Ion emission has been observed indirectly from the average charge-to-mass ratio, as discussed in detail in Section 5.4.1. Using a time-of-flight mass spectrometer, Terhune was able to directly observe ion emission while operating a normal-field electrospray source with the same ferrofluid. ${ }^{32}$

\subsection{Conclusions}

The angular current density was measured for a normal-field electrospray source continuously over the course of the entire emission span, permitting the angular power efficiency factor, $\eta_{\theta}$, to be determined. An analysis of the angular power efficiency factor yielded very small, but statistically significant difference between the positive and negative emission polarities. Further analysis demonstrated a strong correlation between increasing emission current and mass flow rate and decreasing angular efficiency. These correlations agree with observations made by previous researchers. However, the relative loss in efficiency is rather small - indicating that this source can be operating over a large range of emission currents with minimal impact on angular efficiency. This would be of interest when designing a propulsion system which will operate at multiple thrust and specific impulse setpoints.

A final correlation analysis performed between the emission current and mass flow

rate, yielding a higher current-flow rate correlation than that predicted by the $I \sim Q^{1 / 2}$ scaling law presented by de la Mora and Loscertales. ${ }^{53}$ However, such a relation is not 
entirely unexpected since the high conductivity of the fluid lies in a range at which ion emission can result. The $Q^{1 / 2}$ scaling law breaks down when ion emission occurs. ${ }^{38}$ 



\section{Chapter 7 Particle Force in the Apex Field}

In the previous chapter, an experimental study was performed with the goal of identifying the influence of the magnetic field, as one of many factors, on the angular divergence and angular power efficiency of a ferrofluid electrospray source. The small sample size of magnetic fields present in this study was insufficiently-small to enable a statistical analysis to be performed to obtain a correlation analysis of magnetic field influences. To further investigate the influence of magnetic field, a numerical analysis is presented in this chapter to approximate the magnitudes of the Kelvin and Lorentz forces in the apex region. This analysis will determine if these forces are sufficiently strong to impact particle trajectories.

First, let us consider the case of a nanoparticle laden droplet emitted in the spray plume, which will be sufficiently small that it will behave akin to a magnetic dipole. The resulting force acting on this particle, referred to as the Kelvin force density, becomes: ${ }^{55}$

$$
\vec{f}_{m}=\mu_{0}(\vec{M} \cdot \vec{\nabla}) \vec{H}_{0}
$$

where $\vec{f}_{m}$ is the Kelvin magnetic force density, $\vec{M}$ is particle magnetization, and $\vec{H}_{0}$ is the magnetizing field strength of the free field, i.e., the $\mathrm{H}$-field at the location of the droplet while neglecting the field contributions from the droplet. An intense gradient in the magnetic field near the fluid apex suggests the Kelvin force may be sufficiently strong to alter particle trajectories.

When electrospray emission is achieved in the presence of a magnetic field, a $q \vec{v} \times \vec{B}$ force emanates, which is expressed in the following Lorentz force equation: 


$$
\vec{F}=q \vec{E}+q \vec{v} \times \vec{B}
$$

If the charge-to-mass ratio of the ion is sufficiently large, the trajectory of the particle will be deflected as the $q \vec{v} \times \vec{B}$ component of the force incites the particle into a circular trajectory spiraling around the magnetic field vector. The magnetic component of the Lorentz force does not depend on the fluid magnetization, and would arise in the case of traditional electrospray if a magnetic field is present. Little could be found in the literature regarding electrospray emission interacting with magnetic fields, and a majority of what could be found involved mass spectrometry systems and required magnetic fields on the order of many Tesla. ${ }^{110,111}$ With a prior familiarity with the magnetic fields necessary to alter the trajectory of high charge-to-mass ratio ions, it is a reasonable hypothesis that even the intense magnetic fields in the apex region will be insufficient to alter the particle trajectory.

\subsection{Goals of Study}

The goal of this short study was to analyze the influence of the Kelvin force and Lorentz force on droplets resulting from electrospray emission in the proximity of the Taylor cone apex to determine how the field conditions impact particle trajectory. Kelvin force modeling will be achieved through the use of a finite element model - permitting the Coulomb and Kelvin forces to be calculated in the apex region. The apex field will be modeled with the use of an empirical relation that closely resembles the Taylor cone geometry. The Lorentz force will be investigated through the analysis the Larmor radius or various mass-to-charge ratio particles in the apex field conditions.

\subsection{Kelvin Force Modeling and Results}

Consider a nanoscale charged fluid droplet. The Rayleigh limit expresses the maximum charge a this droplet can contain and remain stable. ${ }^{112}$ Above this limit, the droplet becomes unstable and will disintegrate into smaller droplets. This limit is given as: 


$$
Q_{r}=2 \pi\left(16 \pi^{2} \varepsilon_{0} \gamma r^{3}\right)^{1 / 2}
$$

where $Q_{r}$ is the Rayleigh charge limit. Not all electrospray droplets are charged to the full Rayleigh limit. The parameter $\Phi \in(0,1)$ denotes the Rayleigh limit charge factor. A value of $\Phi=0.5$ will be assumed here to present a reasonable value of droplet charge based on work by Miller. ${ }^{103}$ The Coulomb force on a droplet becomes:

$$
\vec{F}_{e}=\Phi Q_{r} \vec{E}
$$

Now let us consider a nanoscale single-domain magnetic nanoparticle of volume $\forall$. From the particle volume and the Kelvin force density, presented in Eq. (6.6), the net Kelvin force acting on the particle can be determined. When scaled in respect to the Coulomb force, a force ratio vector can be established:

$$
\vec{\eta}=\frac{\vec{F}_{e}}{\vec{F}_{m}}=\frac{\Phi Q_{r} \vec{E}}{\forall \mu_{0}(\vec{M} \cdot \vec{\nabla}) \vec{H}_{0}}
$$

To obtain a representative comparison of the ratio of Coulomb to Kelvin force in ferrofluid electrospray, two particles will be analyzed. Coulomb forces will be calculated for a $30 \mathrm{~nm}$ spherical fluid droplet charged to $50 \%$ of the Rayleigh limit, $\Phi=0.5$. Kelvin forces will be equivalent on an equal sized spherical single-domain iron oxide particle at the material saturation. The magnetic saturation for magnetite is $4.45 \times 10^{5} \mathrm{~A} / \mathrm{m} .{ }^{55}$

\subsubsection{Modeling Approach}

The geometric domain utilized for this study is based on the emitter apparatus which was presented previously in Section 5.2.1. The geometric model selected for the fluid interface as well as the FEA modeling technique are presented in the following two subsections. 


\subsubsection{Taylor Cone Model}

The fluid interface model presented in the Chapter 4 worked well to predict the fluid interface on a macroscopic scale. For an emitting electrospray source, the geometry in the region of the apex can be on the order of $10 \mathrm{~s}$ of nanometers to micrometers. The interface prediction model that was present is unable to resolve both the macro- and microscopic scales of the fluid interface and thus is incapable of modeling the fluid interface once snapover occurs.

A static model was therefore selected to model the fluid interface based on pervious work published by Krpoun and Shea. ${ }^{75}$ Their approach implemented a Bernstein-Bézier curve to model the Taylor cone. Although this model is non-physical, i.e., the interface stresses do not balance perfectly, it makes an accurate representation of the Taylor cone geometry. The authors implemented this interface model to computationally predict the onset voltage of a traditional electrospray source. In cartesian coordinates, the surface is expressed as:

$$
\begin{gathered}
x(t, w, \phi)=\frac{(1-2 t) p_{x}}{1-2 t(1-t)(1-w)} \cos (\phi) \\
y(t, w, \phi)=\frac{(1-2 t) p_{x}}{1-2 t(1-t)(1-w)} \sin (\phi) \\
z(t, w, \phi)=\frac{2(1-t) t w \cot (\beta) p_{x}}{1-2 t(1-t)(1-w)}
\end{gathered}
$$

where the Taylor cone half angle, $\beta$, is $49.3^{\circ}$ and $p_{x}$ is the base radius of the Taylor cone. In the $\mathrm{XZ}$ plane, the angular coordinate $\phi=0$. The apex radius is:

$$
r_{a}=\frac{p_{x}}{w} \tan (\beta)
$$

For a 1.5-mm radius reservoir, the Bernstein-Bézier model for a Taylor cone is presented in Figure 7.1: 


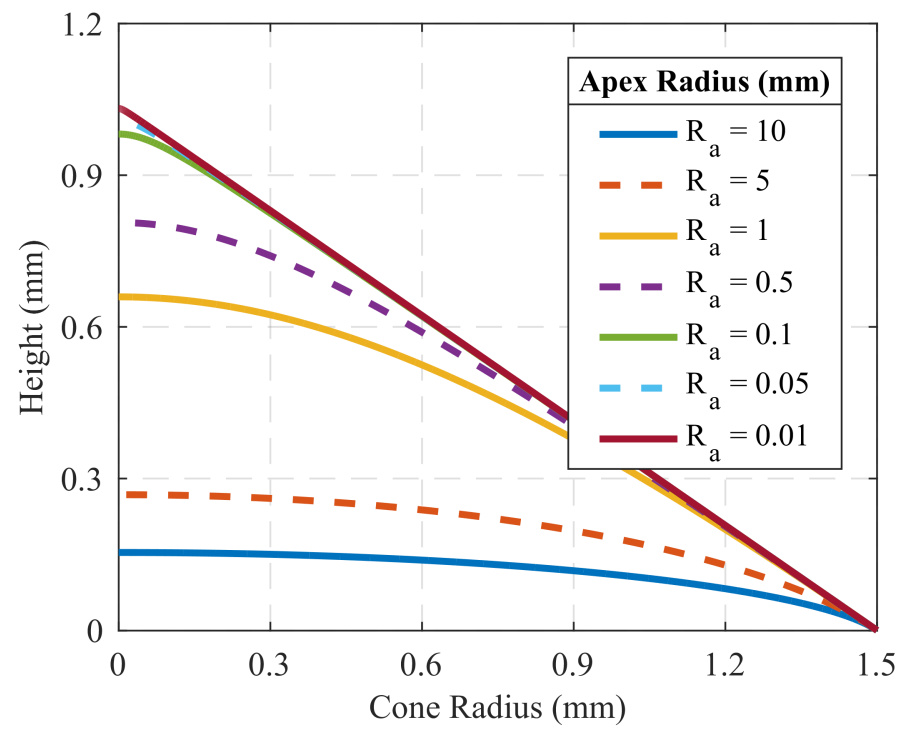

Figure 7.1: Bernstein-Bézier Taylor Cone model with a range of apex radii shown. The half-angle was set to of $49.3^{\circ}$.

For the work presented in this section, the apex radius in the Taylor cone model was set to $1-\mu \mathrm{m}$, yielding an apex electric field of $6 \times 10^{7} \mathrm{~V} / \mathrm{m}$. The apex radius is larger than what is to be expected at the apex of an emitting Taylor cone, however, further refinement of the apex radius will only reinforce the conclusions drawn in the next section.

\subsubsection{FEA Model}

The ferrofluid simulated in this study was treated as a rigid entity having the material magnetic and electrical properties of the ionic liquid ferrofluid discussed in Table 4-1 within Section 4.2. A magnetic field was generated from the presence of two 1 " dia. x 1/8" thick N52 disk magnets. The magnets were modeled with a residual induction, $B r_{\max }$, of 14,800 gauss $^{*}$ with a relative permeability, $\mu_{r}$, of 1.05 . The resulting field at the reservoir interface in the absence of ferrofluid was 520 gauss at the top surface of the fluid reservoir. The actual field for the emitter in this configuration was measured to be 460 gauss.

\footnotetext{
* Provided by KJ Magnetics
} 
The computational domain is presented in Figure 7.2. The fluid interface was partitioned into separate domains near the fluid apex. This enabled the region where the highest gradient exists to be meshed independently, allowing fine control over the mesh in this region. Quartic elements were selected to solve for the magnetic and electric scalar potential. A mesh refinement study was performed in which the concentration of elements in the apex region was increased and decreased by a factor of 2.5. No difference was observed in the first four significant figures of either the magnetic or electric fields.

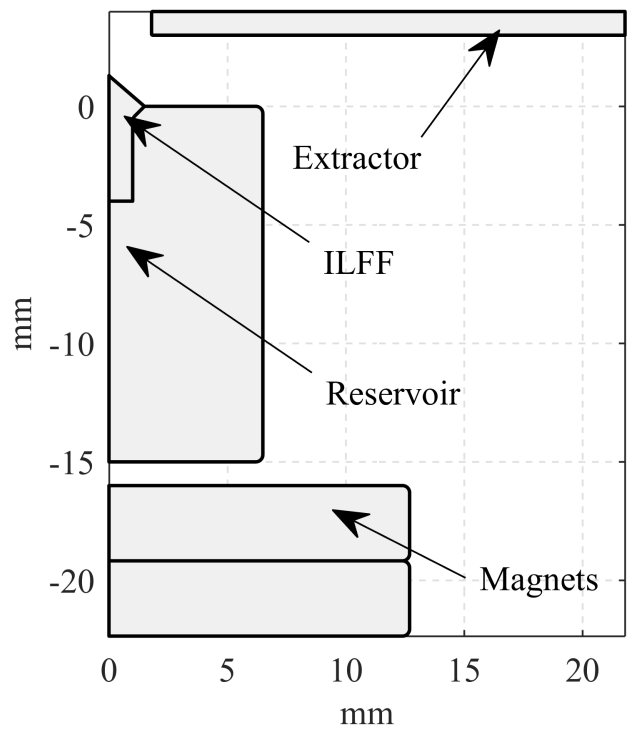

Figure 7.2: Subset of computational domain for FEA. Computational domain was axisymmetric around the y-axis.

When calculating the Kelvin force, uncertainty existed as to the direction of the magnetization vector $\vec{M}$. Within the ferrofluid, it is assumed that the localized magnetization is colinear with $\vec{H}$, a result of the null torsional forces acting on the particle when the two vectors are aligned. ${ }^{\dagger}$ Allowing the particle orientation in this analysis to be that which yielded the greatest Kelvin force would require an optimization to be performed at each node in the FEA domain. A study at select points in the region of analysis showed

$\dagger$ The torque per unit volume on a magnetized particle takes the following form: torque density $=\mu_{0} \vec{M} \times \vec{H}_{0}$ 
that when $\vec{M}$ is colinear with $\vec{H}$, the Kelvin force was at minimum $95 \%$ of that resulting from the orientation which yielded the maximum force, a condition that results from the nearly radial $H$ field in the apex region. Therefore $\vec{M}$ was assumed to be colinear with $\vec{H}_{0}$.

\subsubsection{Kelvin Force Modeling Results}

The electric potential, electric field magnitude, magnetic field magnitude along with the corresponding forces and the force ratio comparison are presented in the following figures: 


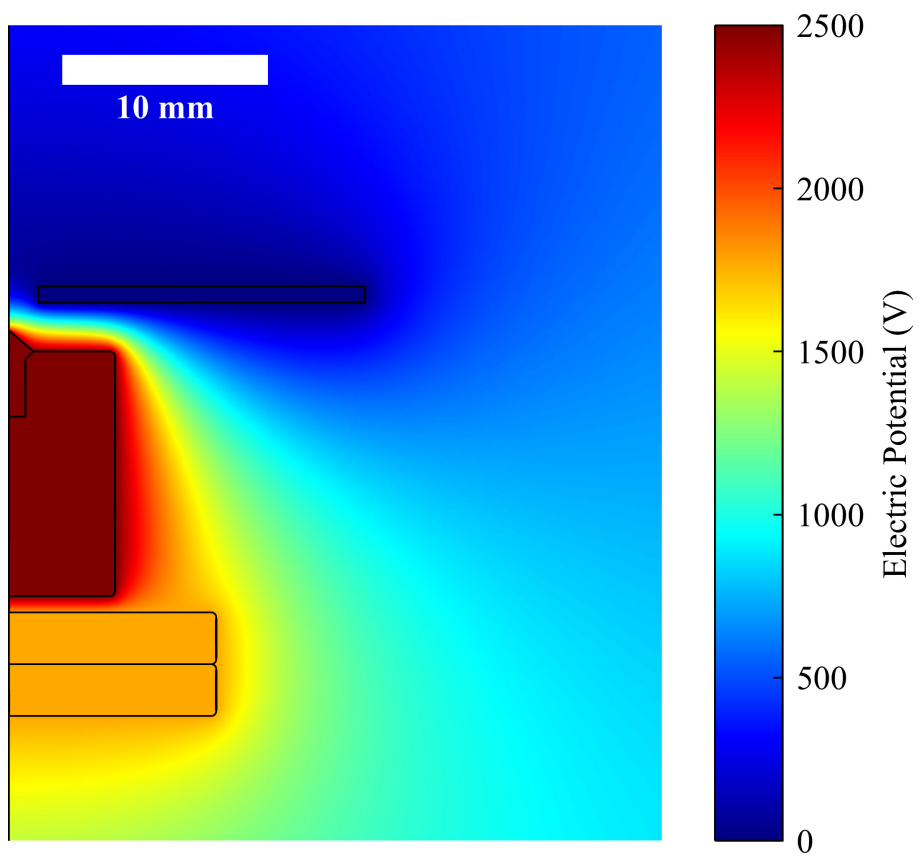

Figure 7.3: Electric potential for the entire emitter geometry.

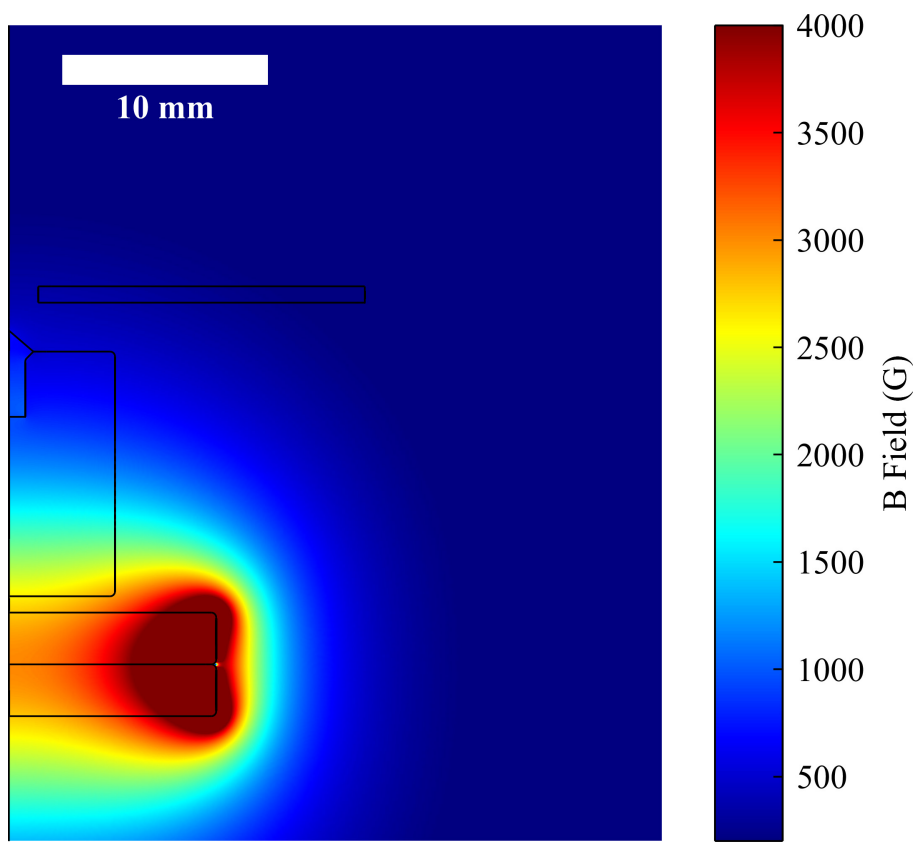

Figure 7.4: Magnetic field within the entire emitter geometry. 


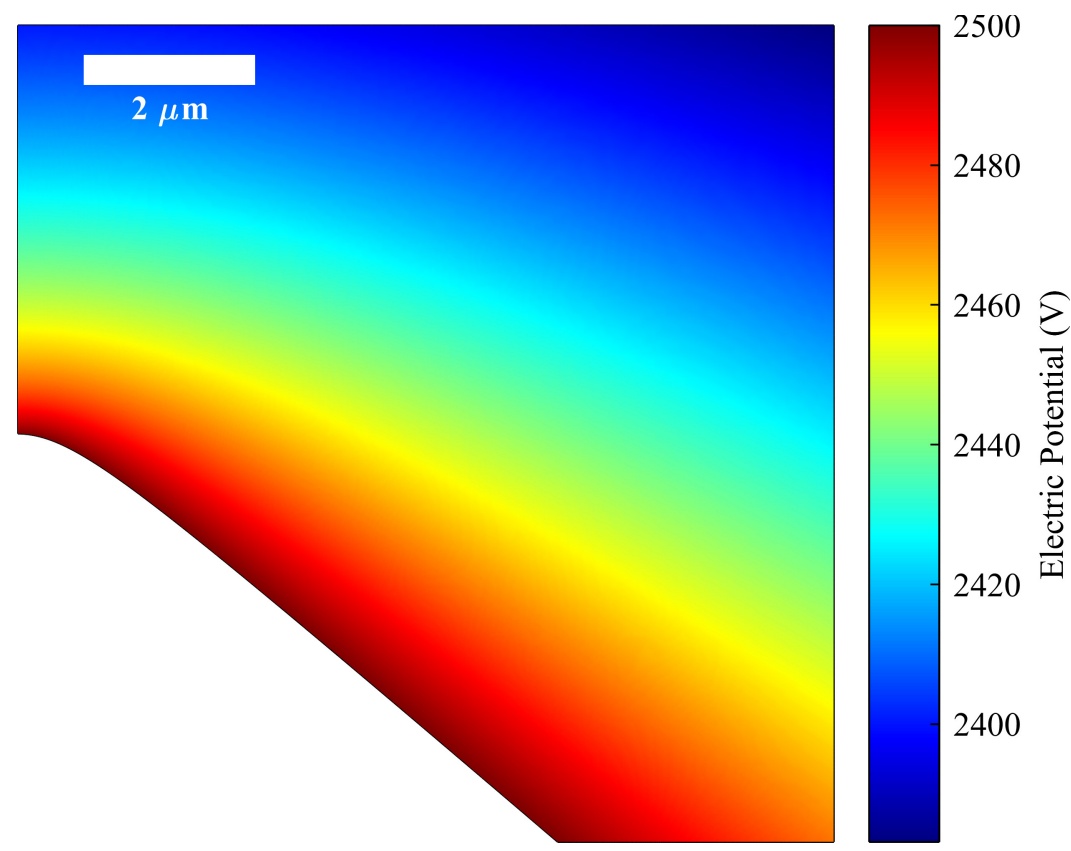

Figure 7.5: Electric potential in the proximity of the Taylor cone apex.

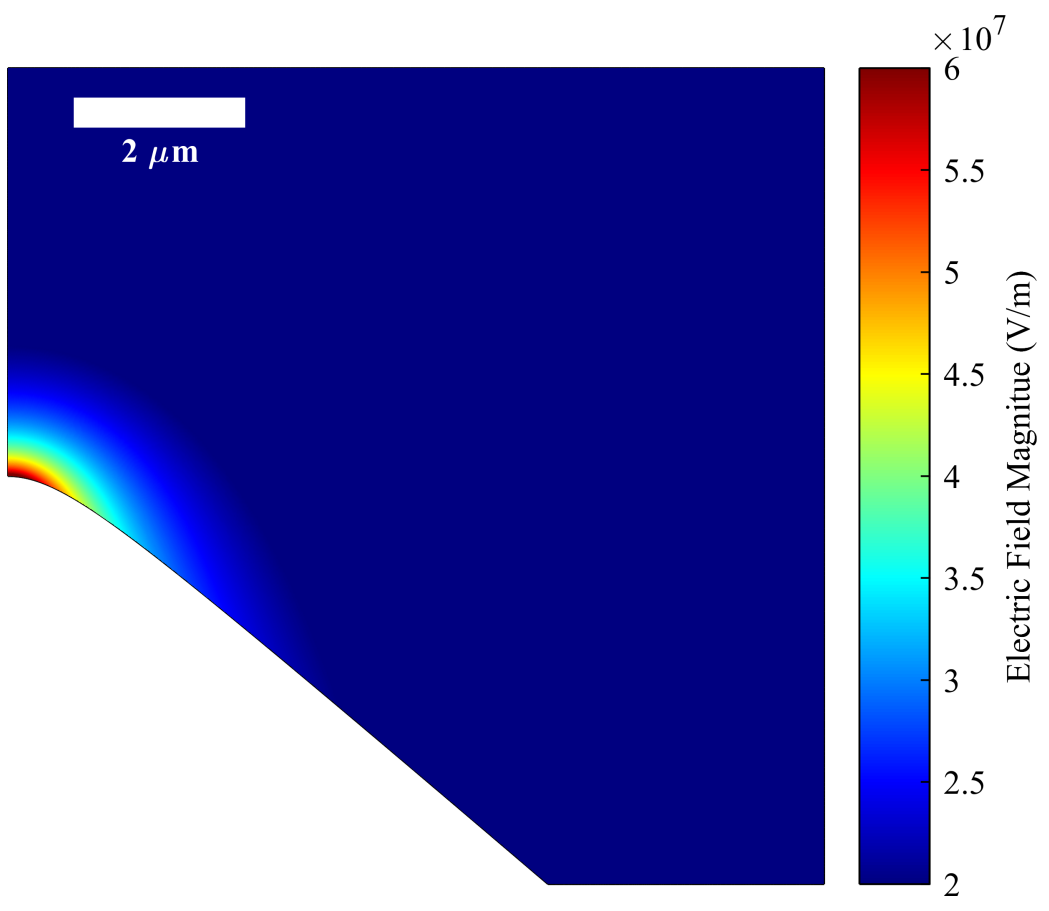

Figure 7.6: Electric field in the proximity of the Taylor cone apex. 


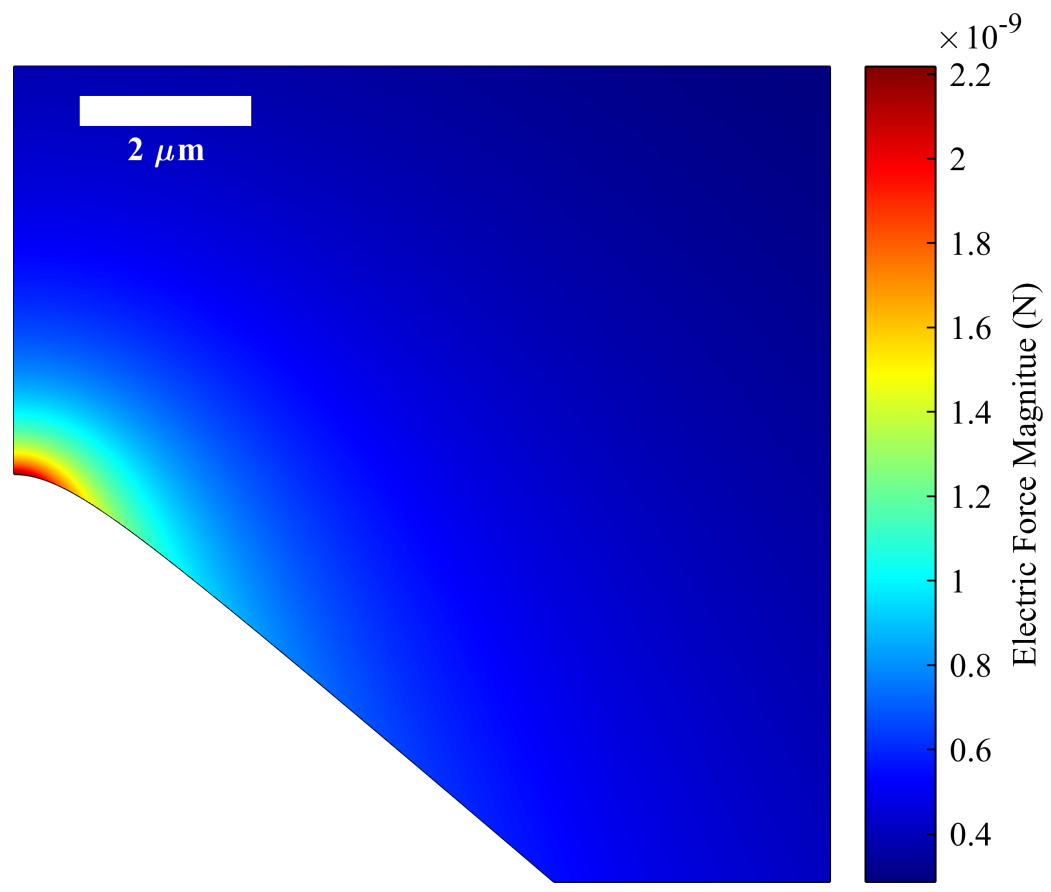

Figure 7.7: Coulomb forces on $30 \mathrm{~nm}$ radius droplet charged to $50 \%$ of the Rayleigh limit in the proximity of the Taylor cone apex.

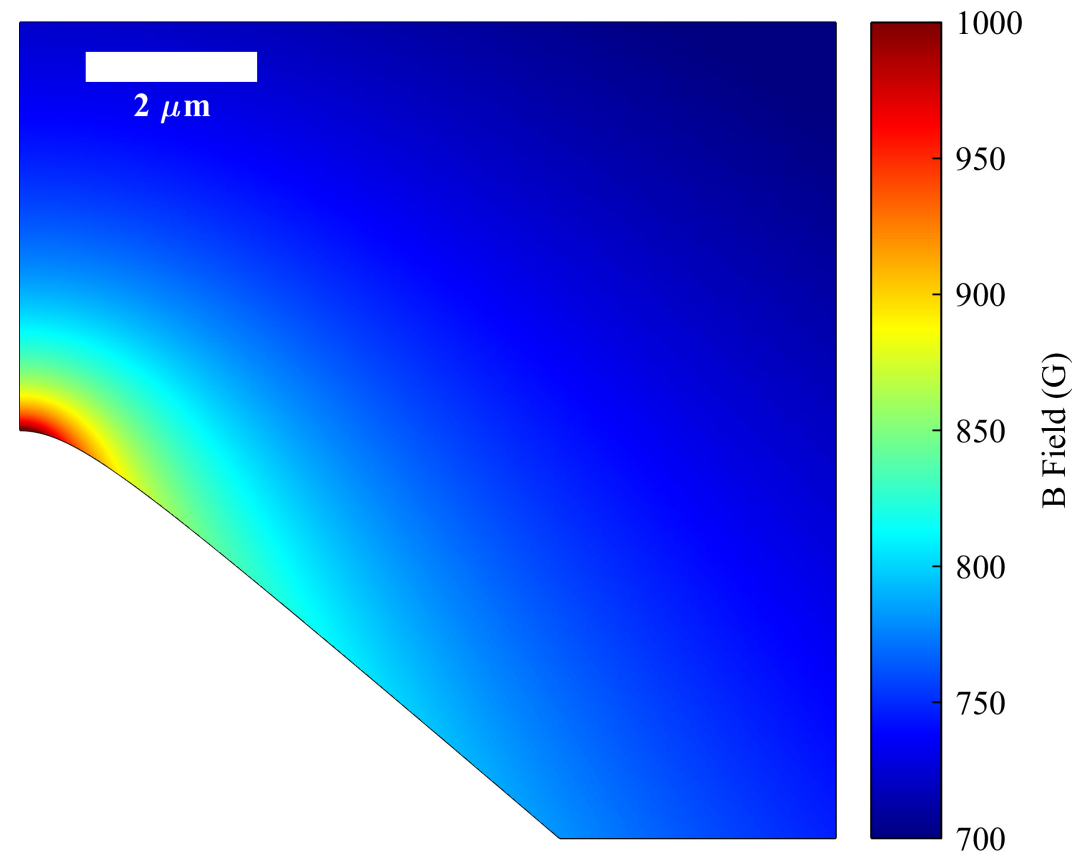

Figure 7.8: Magnetic field strength in in the proximity of the Taylor cone apex. 


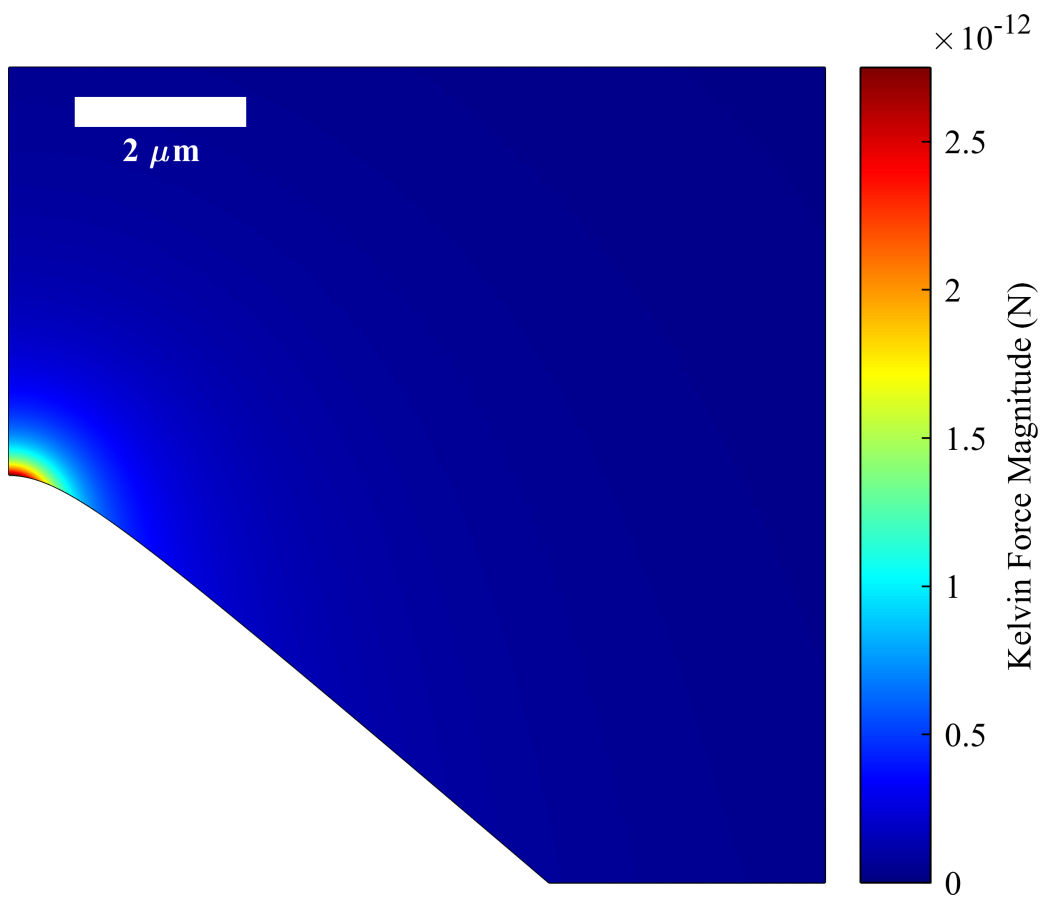

Figure 7.9: Kelvin force on 30-nm radius iron particle at saturation limit of magnetite in the proximity of the Taylor cone apex.

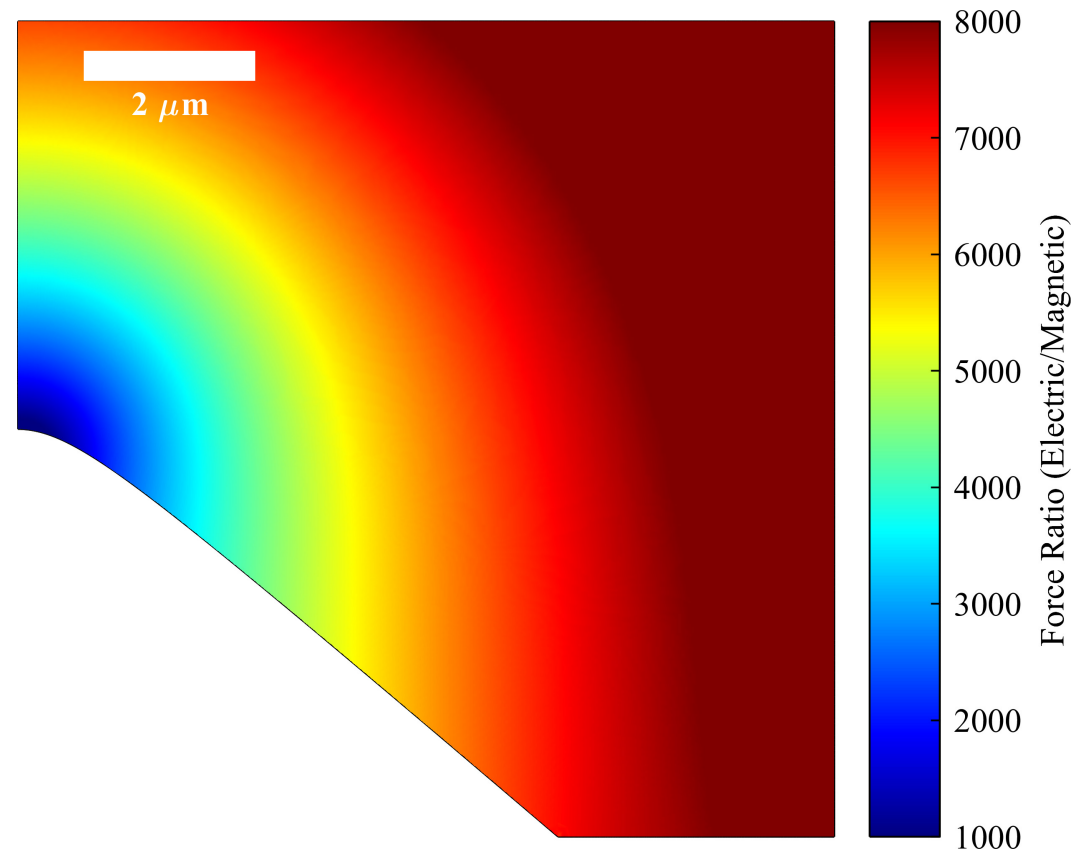

Figure 7.10: Coulomb to Kelvin force ratio near the apex of a Taylor Cone.

From Figure 7.10 it becomes apparent that the Kelvin force acting on a spherical 30$\mathrm{nm}$ radius saturated magnetite particle is considerably smaller than an equivalent sized spherical fluid droplet at $50 \%$ of the Rayleigh limit. The Coulomb force overpowers the 
Kelvin force by three orders of magnitude in the region immediately above the apex, where the ratio is the smallest. The conclusion that can be drawn from this analysis is that the Kelvin force is sufficiently small such that it incapable of altering particle trajectories.

This analysis does make a considerable number of assumptions. These assumptions will be addressed individually for their potential impact.

1. The Taylor cone was set to have an apex radius of $1 \mu \mathrm{m}$ with an applied voltage of $2500 \mathrm{~V}$. The resulting electric field yielded a maximum magnitude of $6 \times 10^{7} \mathrm{~V} / \mathrm{m}$ at the Taylor cone apex. This is below the $\approx 1 \times 10^{9} \mathrm{~V} / \mathrm{m}^{42}$ limit necessary for ion emission. Since ions emitted from the apex will not experience Kelvin forces, only the lower electric field region where droplet emission occurs was modeled.

2. Space-charge effects are not modeled. Space charge effects result when a high density of charged particles or droplets are present in the immediate region of the apex. The combined effect of these droplets reduced the electric field and the resulting Coulomb force.

3. The interface geometry was assumed to follow a Bernstein-Bézier curve. This description is based on cone model for traditional electrospray. This model does not account for magnetic forces. The interface prediction model presented in Sections 4.3.3 and 4.4.3, confirms that near onset, the electric stresses dominate in the immediate proximity to the apex while the magnetic stresses dominate elsewhere. Therefore, the use of the Bernstein-Bézier to approximate the fields in the apex region is an admissible approximation in this region where electric forces dominate by orders of magnitude.

4. Droplet emission results from breakup of the fluid jet. Although the interface is modeled as a cone rather than a cone-jet.

5. The droplet and particle size was assumed to have a radius of $30 \mathrm{~nm}$. Expanding Eq. (6.10) by substituting the volume and Rayleigh limit terms of the radius yields:

$$
\eta=\frac{\Phi 2 \pi\left(16 \pi^{2} \varepsilon_{0} \gamma r^{3}\right)^{1 / 2}|\vec{E}|}{\frac{4}{3} \pi r^{3}\left|\mu_{0}(\vec{M} \cdot \vec{\nabla}) \vec{H}_{0}\right|} \propto \frac{r^{3 / 2}}{r^{3}}
$$


It can be observed that the Kelvin force grows with particle size at a rate of $r^{1.5}$ faster than the Coulomb force. However, the large particle sizes necessary such that Kelvin forces would become comparable are too large to be present within the ferrofluid being considered.

Regardless of these assumptions, any error introduced is unlikely to significantly alter the conclusions drawn from this analysis.

\subsection{Lorentz Force Modeling and Results}

Now consider the case of a charged unmagnetized particle traversing through a magnetic field. Assuming that the electrically charged particle is accelerated up to the electrical potential $V$, the velocity of the particle becomes:

$$
v=\sqrt{2 \frac{q}{m} V}
$$

Consider the scenario where $\vec{v} \perp \vec{B}$ for which the resulting $q \vec{v} \times \vec{B}$ component would be the most drastic. In such a scenario, the particle would begin to rotate around the $\vec{B}$ vector in a circular trajectory (shown in Figure 7.11) which described by the Larmor radius:

$$
r_{L}=\frac{m v_{\perp}}{|q| B}
$$




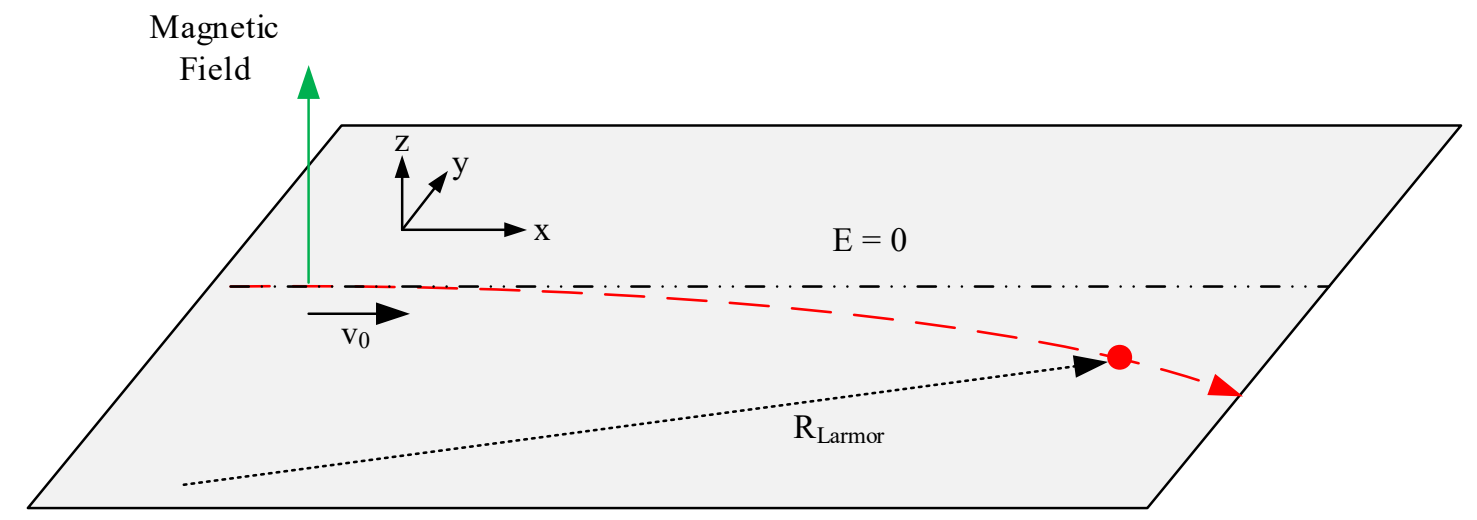

Figure 7.11: In the presence of a uniform magnetic field, qvxB forces the particle will assume a circular trajectory about the magnetic field vector.

In Section 7.2.2, it was shown that in the region directly above the apex of the Taylor cone model, the fluid magnetization approached 0.1 Tesla (1000 gauss). Using this value for $|B|$ and an acceleration potential of $2000 \mathrm{~V}$, the Larmor radius was calculated (shown in Figure 7.12) with respect to the range of estimated mass-to-charge ratios for ionic liquid ferrofluid electrospray measured by Terhune. ${ }^{35}$

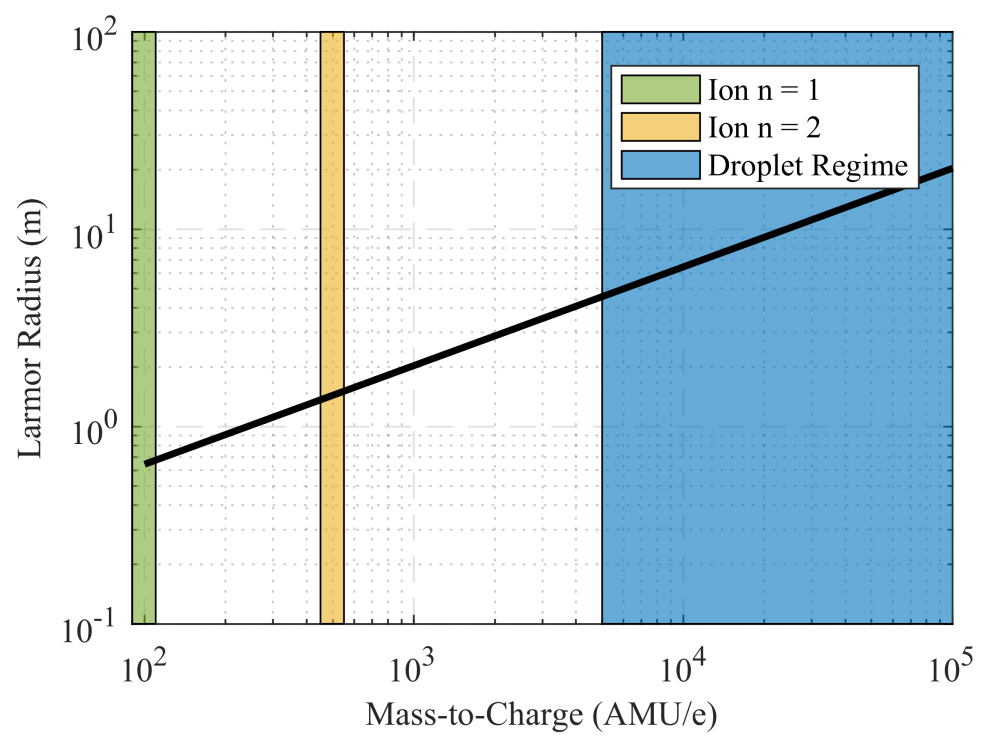

Figure 7.12: Larmor radius for a particle accelerated to $2000 \mathrm{~V}$ within a $0.1 \mathrm{~T}$ magnetic field perpendicular to the initial velocity vector.

The results show that the Larmor radius ( $\sim 1-10$ s of meters) is considerably large for the emitted species relative to scale of the emission source ( $\sim 10$ s of millimeters $)$ and is thus too small to be significant factor on particle trajectory. In practice, $\vec{v}$ and $\vec{B}$ are not 
perpendicular, and $|B|$ decays rapidly when traveling away from the apex. Therefore, this model over estimates the influence of the Lorentz force.

\subsection{Conclusions}

The numerical analysis presented in this chapter reveals the Kelvin force is on the scale of 3 orders of magnitude smaller than the Coulomb force in the apex region. An analysis of the Lorentz force in the apex region showed that the $q \vec{v} \times \vec{B}$ force is insufficient to cause any significant deviation in the particle trajectories of even the highest charge-to-mass ratio ions. These findings were not entirely unexpected. However, the elimination of the Kelvin and Lorentz force on particle trajectories permits future analyses of the divergence of magnetic and colloidal electrosprays to focus on the meniscus geometry as the influential factor. 



\section{Chapter 8 Conclusions}

During this research, a relatively new form of electrospray was investigated. The uniqueness of this research lies in the fact that the fluid which was electrosprayed, an ionic liquid ferrofluid is (1) super-paramagnetic, (2) highly-conductive, (3) colloidal, and (4) capable of being emitted without the need of a supporting structure such as a capillary, externally wetted, or porous needle. A combination of any two of these properties would be sufficient to justifiably classify the spray as being in a subclass of electrospray for which there is little in the literature. Although research has previously been performed on ionic liquid ferrofluid electrosprays, prior work focused primarily on demonstrating that spray can be achieved from a combined magneto-electric instability, ${ }^{30,91}$ or focused on the electrospray behavior of these fluids from a capillary emission source. ${ }^{31,33-35}$ The objectives of this research were to: (1) develop a computational model to simulate interface deformation and determine the role magnetic stresses on onset, (2) determine the propulsion efficacy of an ionic liquid ferrofluid normal-field electrospray source, and finally (3) measure the angular divergence of the spray and determine influential factors in on angular efficiency.

The work presented in this dissertation was successful in these objectives. Technical summaries of the individual research topics are presented in their respective chapters. However, during the course of this research, discoveries were made that span multiple chapters or have impacts on broader electrospray research. This section serves to summarize these discoveries. 


\subsection{Electric Propulsion Viability}

A primary objective this research was to analyze the performance capabilities and viability of an ionic liquid ferrofluid electrospray source as a means for electric propulsion for nanosatellites. In (Page 143) performance parameters of the normal-field electrospray source investigated in this dissertation are presented alongside two developed electrospray thrusters for which published data is available to obtain derived propulsion parameters. Several methods are available to obtain derived propulsion performance parameters. The first method is presented in Section 5.4.2 and uses average values of mass flow rate, emission current, and extraction voltage. Additional methods include time-of-flight mass spectrometer (ToF) or thrust stand measurements. Using a thrust stand, both thrust and specific impulse can be measure directly - yielding estimates that are likely more representative of thruster performance than those determined using less direct methods.

Overall, the single emitter ionic liquid ferrofluid electrospray source studied in this work capable of achieving favorable performance. On per emitter basis, the normal-field source was able to achieve an order of magnitude greater thrust than the MIT iEPS thruster while obtaining a similar specific impulse. Compared to the UC-Irvine thruster, the normalfield source achieved comparable thrust per emitter with a three-fold increase in specific impulse. However, both the MIT an UC-Irvine thrusters are cable of achieving impressive emitter densities, with 480 and 100 emitters $/ \mathrm{cm}^{2}$, respectively. The packing density of normal-field emitters as well as the ability to maintain emission from multiple emitters simultaneously remains unknown. However, performance parameters derived from this work lends credence to the concept of this type of electrospray and findings presented herein serve to justifies further efforts to refine the technology. 
Table 8-1: Propulsion performance parameters of the ionic liquid ferrofluid normal-field electrospray source alongside those of developed propulsion systems.

\begin{tabular}{|c|c|c|c|c|c|c|}
\hline & & ILFF & \multicolumn{2}{|c|}{ MIT $^{\text {iEPS }}{ }^{1}$} & \multicolumn{2}{|c|}{ UC-Irvine $^{2}$} \\
\hline & Propulsive Fluid & $\begin{array}{c}\text { EMIM-NTf2 } \\
\text { Ferrofluid }\end{array}$ & \multicolumn{2}{|c|}{ EMI-BF4 } & \multicolumn{2}{|c|}{ EMIM-NTf2 } \\
\hline & Method & $\dot{\bar{m}}, \bar{I}$, and $V$ & $\begin{array}{c}\dot{\bar{m}}, \bar{I} \text {, and } \\
V\end{array}$ & $\begin{array}{c}\text { ToF Methods } \\
\text { and Thrust } \\
\text { Stand }\end{array}$ & $\begin{array}{c}\dot{\bar{m}}, \bar{I} \text {, and } \\
V\end{array}$ & ToF Methods \\
\hline Thrust & $(\mu \mathrm{N})$ & 0.38 & $19.4^{*}$ & $11-12.5$ & $10.2-69 *$ & $8.1-54$ \\
\hline$I_{s p}$ & (s) & $1200-1400$ & 1150 & 760 & $123-297^{*}$ & $96-236$ \\
\hline $\begin{array}{l}\text { Thrust/ } \\
\text { Power }\end{array}$ & $(\mu \mathrm{N} / \mathrm{W})$ & 154 & 144 & 87 & $690-1650^{*}$ & $\begin{array}{c}541- \\
1300^{*}\end{array}$ \\
\hline Voltage & $(\mathrm{kV})$ & 3.33 & \multicolumn{2}{|c|}{0.90} & \multicolumn{2}{|c|}{1.76} \\
\hline Current & $(\mu \mathrm{A})$ & 0.75 & \multicolumn{2}{|c|}{150} & \multicolumn{2}{|c|}{$8.5-23.6$} \\
\hline Power & $(\mathrm{mW})$ & 2.5 & \multicolumn{2}{|c|}{135} & \multicolumn{2}{|c|}{$15-41.5$} \\
\hline$\eta_{\theta}$ & -- & 0.94 & \multicolumn{2}{|c|}{0.81} & \multicolumn{2}{|c|}{--} \\
\hline Emitters & -- & 1 & \multicolumn{2}{|c|}{480} & \multicolumn{2}{|c|}{64} \\
\hline Thrust/Emitter & $(\mu \mathrm{N})$ & 0.38 & $0.040^{*}$ & $0.023-0.026$ & $0.16-1.1$ & $0.13-0.84$ \\
\hline Power/Emitter & $(\mathrm{mW})$ & 0.4 & \multicolumn{2}{|c|}{0.28} & \multicolumn{2}{|c|}{$0.23-0.65$} \\
\hline
\end{tabular}

* These values were derived for this purpose using measurements provided by the respective authors within their publication. They were not reported directly by the authors. 


\subsection{Electrically and Magnetically Stressed Fluid Surface Modeling Techniques}

In Chapter 4, a literary review was presented on previous work on electrohydrodynamic, ferrohydrodynamic, and paramagnetic modeling of freely suspended or pinned sessile drops. Of the work reviewed, a majority employed semi-analytical ${ }^{76-78,80 \text {, }}$ 82 or finite element methods ${ }^{84-86}$ that did not consider fluid motion. Only three sources in the literature were found that employed finite element methods capable of solving for arbitrary geometries while resolving internal fluid motion. ${ }^{81,87,89}$

The modeling technique presented in this dissertation is the only work known to the author to exist in the literature that presents a methodology to simulate surface and fluid motion under combined ferrohydrodynamic and electrohydrodynamic stresses. The mesh tracking technique incorporated is capable of very accurately tracking the interface movement and stresses under the limitation that the fluid does not experience topological changes (i.e. breakup). The developed methodology with slight modifications can serve to analyze several remaining questions regarding ionic liquid ferrofluid electrospray and within the broader electrospray field. The presented work utilizes a commercially available code which permits additional physics, such as ohmic heating, bulk and surface charge transport, or any custom differential equation can be added to the physics with ease.

One area of electrospray physics for which a large uncertainty remains is the cone-jet transition region. The physics of this region govern emission current, flow rate, and jet diameter. A numerical model of this region would help to (1) clarify the scaling the laws in electrospray and (2) reduce reliance on extrapolation of scaling laws to unexplored electrospray regimes.

One pressing matter in the expansion of electrospray from a normal-field instability is the expansion of spray from a single to multiple emission sites. During early research into ionic liquid ferrofluid emitters, both Meyer ${ }^{91}$ and den Hartog ${ }^{113}$ performed experiments using multipeak instabilities and faced challenges getting multiple peaks to emit. Both authors observed a phenomenon where only one tip would begin emitting. Further increasing the extraction potential would permit additional peaks to emit, however, the first 
emitter would transition into an nonideal high-flow rate jetting mode. It is the current hypothesis of the author that differences in the magnetic and electric stresses between peaks is resulting in fluid transfer between instabilities - permitting one to grow at the sacrifice of a neighboring peak. Implementation of the developed modeling technique would permit further analysis into this issue. 



\section{Appendix A Electric Stress Component Derivations}

The following are the complete derivations of the components of the interface surface stress. The following assumptions will be made: (1) only one fluid exhibits a relative

permittivity $\left(\varepsilon_{r}\right)$ greater than one, (2) the fluids are incompressible, (3) the fluids are isothermal.

Note: These derivations utilize the incompressible non-linear form of the fluid stress tensor.

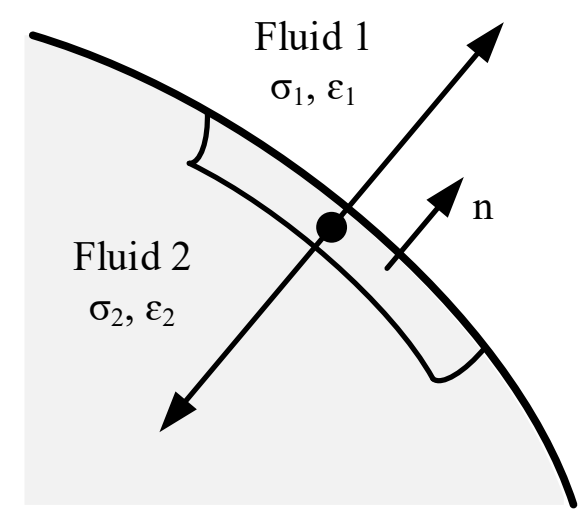

Figure A.1: Fluid interface of high permittivity fluid (Fluid 2) with low permittivity fluid (Fluid 1).

\section{A1. Surface Stress Components}

For an incompressible nonlinear medium the stress tensor is: ${ }^{114}$ 


$$
\mathbf{T}_{e}=\left\{-\vec{E} \cdot \vec{D}+\int_{0}^{\vec{D}} \vec{E} \cdot d \vec{D}\right\} \mathbf{I}+\vec{D} \vec{E}
$$

Assuming the material is linear $(\vec{D}=\varepsilon \vec{E})$, the integral term can be expressed as follows:

$$
\mathbf{T}_{e}=-\frac{1}{2} \varepsilon E^{2}+\varepsilon \vec{E} \vec{E}
$$

The electric stress vector along the interface becomes:

$$
\vec{t}_{n}=\hat{n} \cdot \mathbf{T}_{e}=-\frac{1}{2} \varepsilon E^{2} \hat{n}+\varepsilon E_{n} \vec{E}
$$

\section{A2. Normal Stress Component}

The normal component of this stress vector can be determined as follows:

$$
\vec{t}_{n} \cdot \hat{n}=-\frac{1}{2} \varepsilon E^{2} \hat{n} \cdot \hat{n}+\varepsilon E_{n} \vec{E}_{n}
$$

The normal component of the surface traction thus becomes:

$$
\begin{aligned}
t_{n} \cdot \hat{n} & =\hat{n} \cdot\left[T_{e}\right] \cdot \hat{n} \\
& =-\frac{1}{2} \varepsilon E^{2}+\varepsilon E_{n} E_{n}
\end{aligned}
$$

The difference in the normal component of the electric surface traction becomes the normal interfacial stress:

$$
\begin{aligned}
\sigma_{n, \text { elec }} & =t_{e, 1} \cdot \hat{n}-t_{e, 2} \cdot \hat{n} \\
& =-\frac{1}{2} \varepsilon_{1} E_{1}^{2}+\varepsilon_{1} E_{n, 1}^{2}+\frac{1}{2} \varepsilon_{2} E_{2}^{2}-\varepsilon_{2} E_{n, 2}^{2}
\end{aligned}
$$

In the case where fluid 1 is an insulator and fluid 2 is a highly conductive medium, $E_{2}$ becomes zero. The resulting stress is therefore: 


$$
\sigma_{n, \text { elec }}^{\text {cond }}=\frac{1}{2} \varepsilon_{1} E_{1}^{2}
$$

Note, in the case that in the case where fluid 2 is a conductor, $E_{2}=0$ and consequently $E_{t, 2}=0$. Since $E_{t, 1}=E_{t, 2}$ per boundary conditions, $E_{2}=E_{n, 2}$.

\section{A3. Tangential Stress Component}

The normal component of the surface traction thus becomes:

$$
\begin{aligned}
\vec{t}_{n} \cdot \hat{t} & =\hat{n} \cdot \mathbf{T}_{e} \cdot \hat{t} \\
& =-\frac{1}{2} \varepsilon E^{2} \hat{n} \cdot \hat{t}+\varepsilon E_{n} E_{t}
\end{aligned}
$$

In the previous equation, the scalar component $-(1 / 2) \varepsilon E^{2} \hat{n}$ is null since $\hat{n} \cdot \hat{t}=0$.

$$
\begin{aligned}
\sigma_{t, \text { elec }} & =\vec{t}_{e, 1} \cdot \hat{t}-\vec{t}_{e, 2} \cdot \hat{t} \\
& =\varepsilon_{1} E_{1, n} E_{1, t}-\varepsilon_{2} E_{2, n} E_{2, t}
\end{aligned}
$$

Recalling, in the absence of surface charge: $E_{1, T}=E_{2, T}$ and $\varepsilon_{1} E_{1, n}=\varepsilon_{2} E_{2, n}$, the resulting stress becomes:

$$
\sigma_{t, \text { elec }}=0
$$

In the case of a perfect conductor, the internal electric field and tangential electric field is zero. Likewise, the surface stress becomes zero. These results are independent of the form of the stress tensor utilized. 



\section{Appendix B Magnetic Stress Component Derivations}

The following are the complete derivations of the components of the interface surface stress. The following assumptions will be made: (1) only one fluid exhibits magnetic properties, (2) the fluids are incompressible, (3) the fluids are isothermal.

Note: These derivations utilize the incompressible non-linear form of the fluid stress tensor.

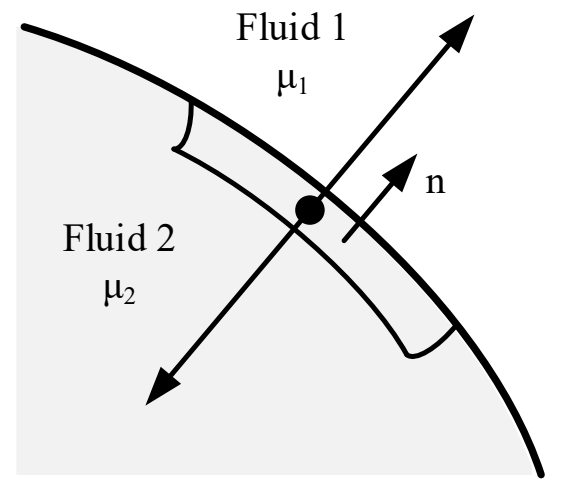

Figure B.1: Fluid interface of ferrofluid (Fluid 2) with non-magnetic fluid (Fluid 1).

\section{B1. Surface Stress Components}

Consider the stress tensor for a non-linear incompressible ferrofluid (Eq. (3.5)): ${ }^{.5}$

$$
\mathbf{T}_{m}=-\left\{\mu_{0} \int_{0}^{H} M d H+\frac{1}{2} \mu_{0} H^{2}\right\} \mathbf{I}+\vec{B} \vec{H}
$$

The stress vector along the interface becomes: 


$$
\vec{t}_{n}=\hat{n} \cdot \mathbf{T}_{m}=-\left\{\mu_{0} \int_{0}^{H} M d H+\frac{1}{2} \mu_{0} H^{2}\right\} \hat{n}+B_{n} \vec{H}
$$

\section{B2. Normal Stress Components}

The normal component of this stress vector can be determined as follows:

$$
\vec{t}_{n} \cdot \hat{n}=-\left\{\mu_{0} \int_{0}^{H} M d H+\frac{1}{2} \mu_{0} H^{2}\right\} \hat{n} \cdot \hat{n}+B_{n} H_{n}
$$

If fluid 1 is a non-magnetic medium, then the difference in the normal stress vector over the interface becomes:

$$
\begin{aligned}
\sigma_{n, \text { mag }} & =\vec{t}_{n, 1} \cdot \hat{n}-\vec{t}_{n, 2} \cdot \hat{n} \\
& =-\frac{1}{2} \mu_{0} H_{1}^{2}+B_{n, 1} H_{n, 1}+\mu_{0} \int_{0}^{H} M d H+\frac{1}{2} \mu_{0} H_{2}^{2}-B_{n, 2} H_{n, 2} \\
& =\frac{1}{2} \mu_{0}\left(H_{2}^{2}-H_{1}^{2}\right)+\left(B_{n, 1} H_{n, 1}-B_{n, 2} H_{n, 2}\right)+\mu_{0} \int_{0}^{H} M d H
\end{aligned}
$$

The following substitution can be made (since $H_{t, 1}=H_{t, 2}$ from the interfacial boundary conditions):

$$
\begin{aligned}
H_{2}^{2}-H_{1}^{2} & =\left(H_{n, 2}^{2}+H_{t, 2}^{2}\right)-\left(H_{n, 1}^{2}+H_{t, 1}^{2}\right) \\
& =H_{n, 2}^{2}-H_{n, 1}^{2}
\end{aligned}
$$

Finally, given that:

$$
H_{n, 1}=\frac{B_{n, 1}}{\mu_{0}}, \quad H_{n, 2}=\frac{B_{n, 2}}{\mu_{0}}-M_{n, 2}
$$

Substituting these relations into Eq. and simplifying, yields: 


$$
\begin{aligned}
\sigma_{n, \text { mag }}= & \frac{1}{2} \mu_{0}\left(\frac{B_{n}^{2}}{\mu_{0}^{2}}-2 \frac{B_{n} M_{n, 2}}{\mu_{0}}+M_{n, 2}^{2}-\frac{B_{n}^{2}}{\mu_{0}^{2}}\right) \\
& +B_{n}\left(\frac{B_{n}}{\mu_{0}}-\frac{B_{n}}{\mu_{0}}+M_{n, 2}\right)+\mu_{0} \int_{0}^{H} M d H \\
= & \underbrace{\frac{1}{2} \mu_{0} M_{n, 2}^{2}}_{1}+\underbrace{\mu_{0} \int_{0}^{H} M d H}_{2}
\end{aligned}
$$

As can be seen, two components arise in the normal component of the magnetic stress on the fluid interface. The first component is the magnetic normal pressure and the second turn is the fluid magnetic pressure.

\section{B3. Tangential Stress Components}

The tangential component of this stress can be determined as follows:

$$
\vec{t}_{n} \cdot \hat{t}=\left\{\mu_{0} \int_{0}^{H} M d H+\frac{1}{2} \mu_{0} H^{2}\right\} \hat{n} \cdot \hat{t}+B_{n} H_{t}
$$

Recognizing that $\hat{n} \cdot \hat{t}=0$, the previous equation can be simplified to:

$$
\begin{aligned}
\vec{t}_{n} \cdot \hat{t} & =\left\{\mu_{0} \int_{0}^{H} M d H+\frac{1}{2} \mu_{0} H^{2}\right\} \hat{n} \cdot \hat{t}+B_{n} H_{t} \\
& =B_{n} H_{t}
\end{aligned}
$$

Finally, the normal component $B_{n}$ and tangential component $H_{t}$ are continuous over the fluid interface; therefore:

$$
\begin{aligned}
\sigma_{t, \text { mag }} & =\vec{t}_{n, 1} \cdot \hat{t}-\vec{t}_{n, 2} \cdot \hat{t} \\
& =B_{n, 1} H_{t, 1}-B_{n, 2} H_{t, 2} \\
& =0
\end{aligned}
$$

This result is independent of the form of the stress tensor utilized. 



\section{Appendix C Methodology of Processing Time- lapse Imagery to Estimate Mass Flow Rate}

Mass flow rates of the single tip ionic liquid ferrofluid emitter were estimated by analyzing time-lapse imagery. Images of the emitter were taken using an OptixCam Summit K2 5.1 MP USB camera integrated with a stereo microscope at 30 second intervals. The emitter was imaged in in grayscale and saved in a .tiff format.

The following methodology was utilized to approximate the volume of the tip:

1. The top of the fluid reservoir was selected via user input. (First image only)

2. The extractor diameter was selected via user input which was used to set a global scale for the image. (First Image only)

3. Each image was converted to black and white using an inputted threshold value.

4. Black-white boundaries were detected and an algorithm was employed to determine which boundary was associated with the ferrofluid.

5. The reservoir boundary was split into the left and right half and each half was integrated to determine the respective volume.

Edges were detected using the bwboundaries function in MATLAB which is based on the Moore-Neighbor tracing algorithm. The average volume was then plotted against time and fit using a spline curve. The derivative of this curve was then used to estimate volume and mass flow rates. 
A sample image with the detected boundaries is presented in Figure C.1. The estimated volume during a long duration test obtained by analyzing a time-lapse of images is presented in Figure C.2.
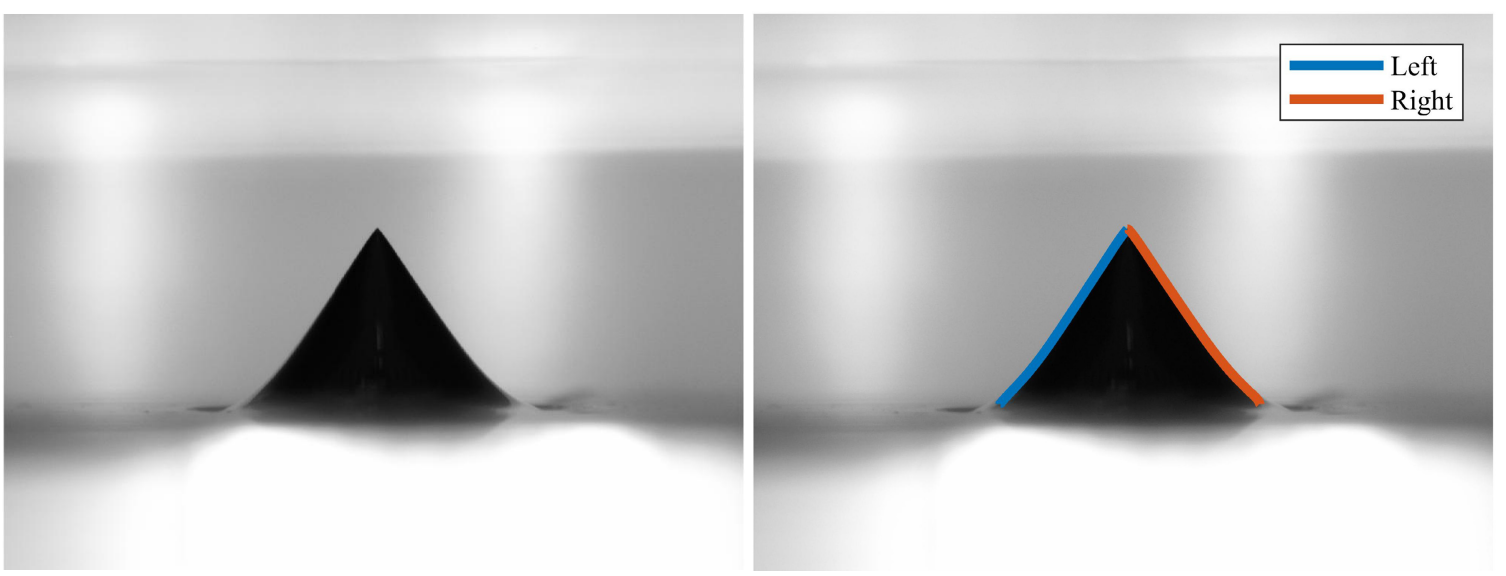

Figure C.1: Typical image acquired through the imaging setup. (Right) Boundaries detected through the image processing algorithm. Left and Right boundaries were integrated to determine peak volume. The average of these volumes was utilized to determine the mean volume.

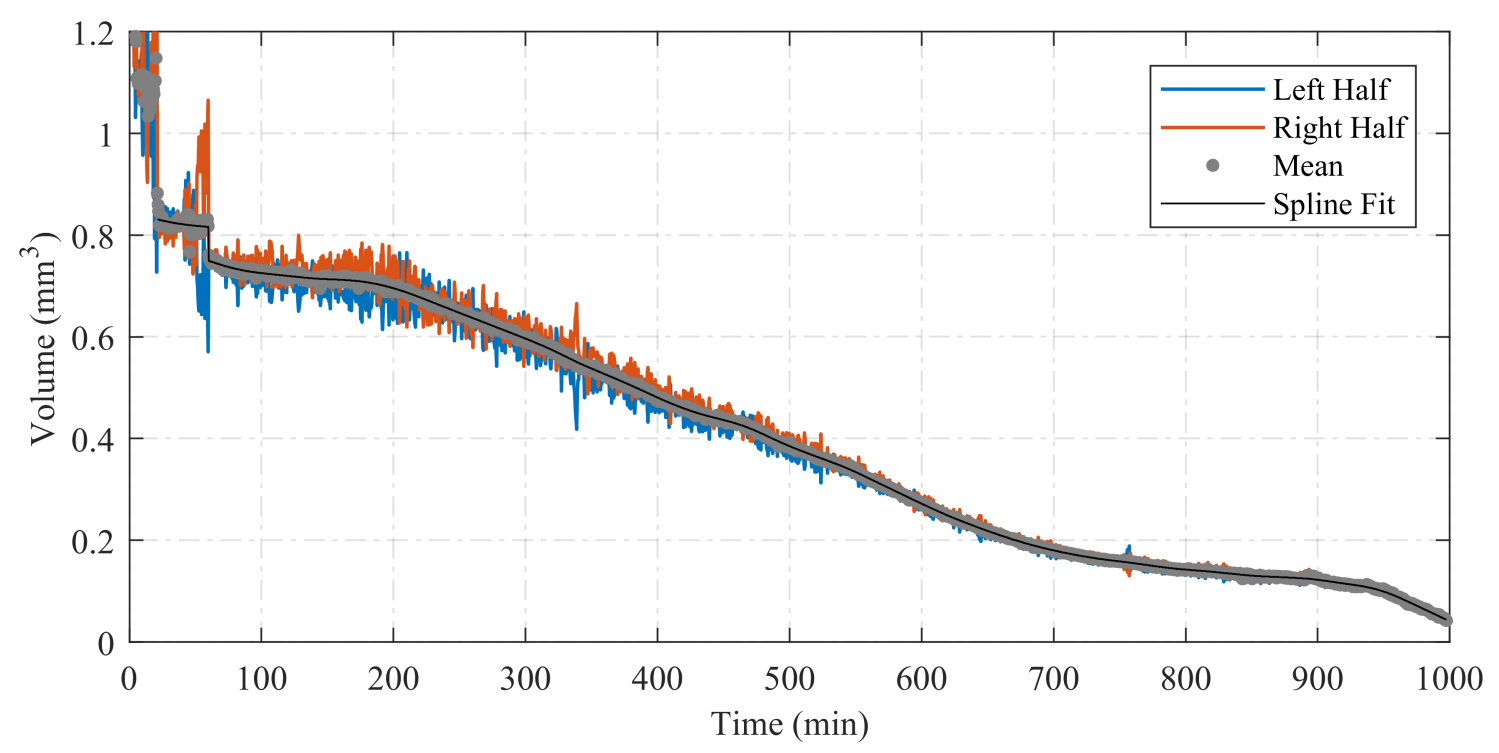

Figure C.2: Spline fit performed on the average of the left and right half integrated volumes. During startup, the source would occasionally spray in a high current mode, which would saturate the current collector. If this occurred, the source was shut down and restarted, resulting in an abrupt change in volume as can be observed above. In this event, separate spline fits were performed before and after the event. 


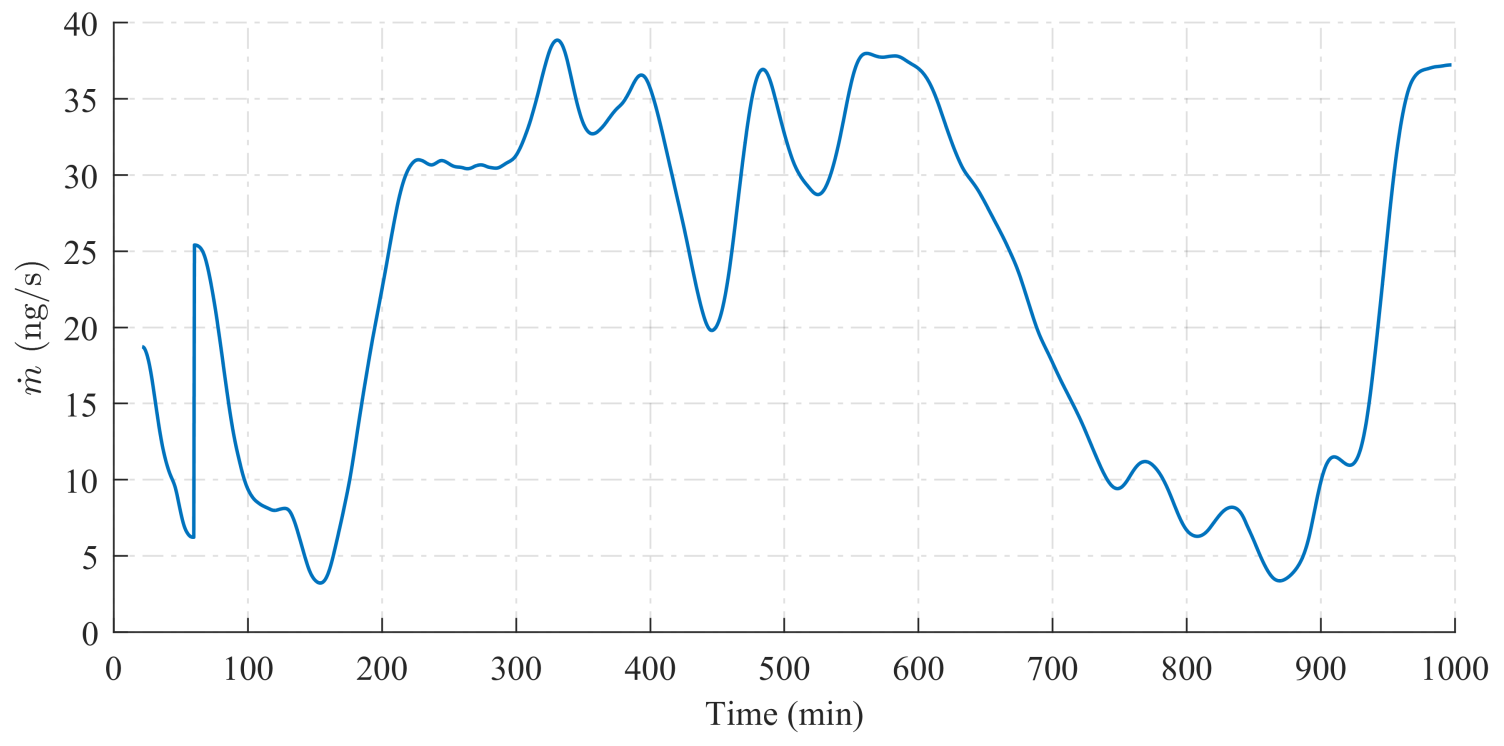

Figure C.3: Mass flow rate obtained by differentiating the volumetric fit spline. 



\section{Appendix D Initial Angular Divergence Studies via Rotating Faraday Collector}

I performed an initial attempt to perform angular divergence measurements of an ionic liquid ferrofluid emission which was presented in 2014 at the 50th AIAA/ASME/SAE/ASEE Joint Propulsion Conference \& Exhibit. ${ }^{105}$ The approach employed for this study rotated a Faraday probe over an emission source and collected in $0.9^{\circ}$ increments within the emission plume. A diagram of the experimental setup is presented in Figure D.1 and Figure D.2.

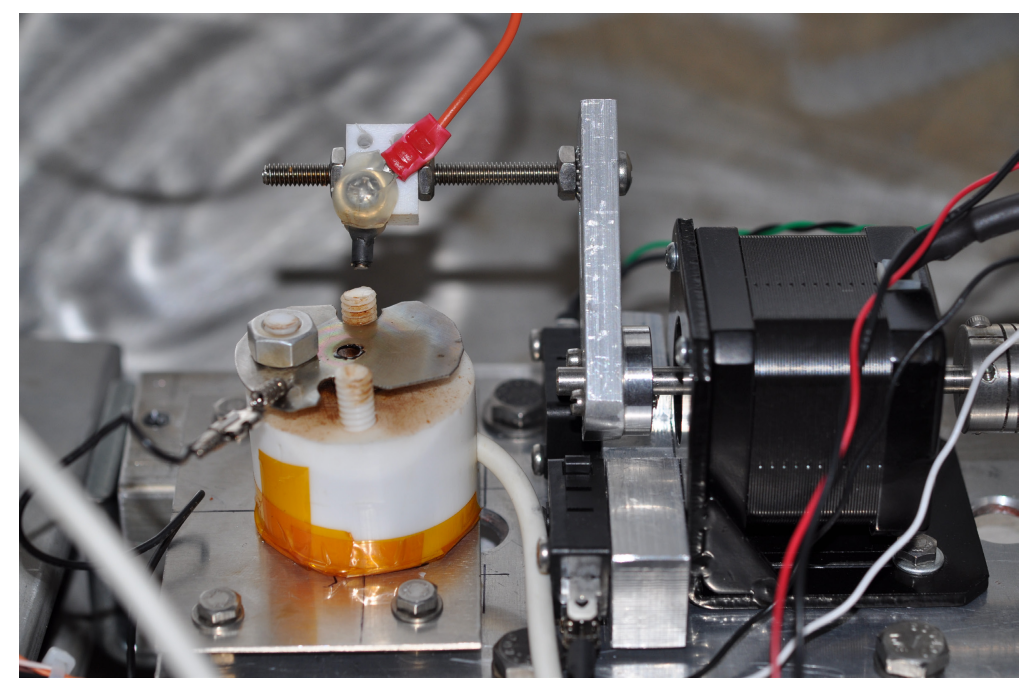

Figure D.1: Experimental setup for initial angular divergence studies. 


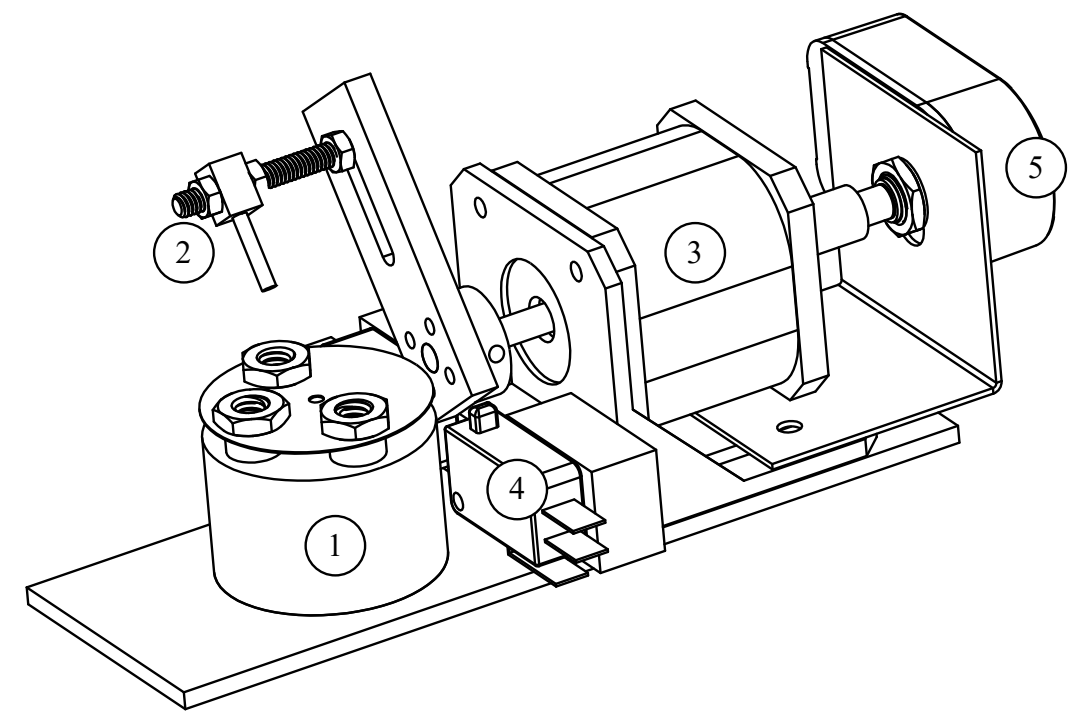

Figure D.2: Diagram of initial angular divergence measurement apparatus. Spray current from the emission source (1) was measured by a Faraday probe (2) mounted on a rotation arm controlled by a servo motor (3). Limit switches (4) were included to position the arm at the datum location and an angular encoder (5) was used to measure the position of the arm.

The goal of this research was to test a new approach for obtaining current distribution measurements for an electrospray while using a normal-field source. The findings and limitations from this study motivated the multiple collector simultaneously reading approach employed within this dissertation.

To perform a collection sequence, an initial angular alignment was obtained by driving the collector arm into a hard stop which was $90^{\circ}$ off axis of the emission centerline. The setup would then proceed to rotate the Faraday probe over the emission site in a semicircle with the base of the circle align with the top of the ferrofluid reservoir. Measurements were taken in one-half step increments, corresponding to $0.9^{\circ}$. At each angular location 100 samples were taken at $1000 \mathrm{~Hz}$ before proceeding to the next measurement point. A complete sweep of the measurement zone would take approximately 25 seconds. This process was repeated for 2 and 3 magnet excitations, corresponding to 115 and 230 gauss, respectively. A sample collected emission plume is shown in Figure D.3 and Figure D.4. 


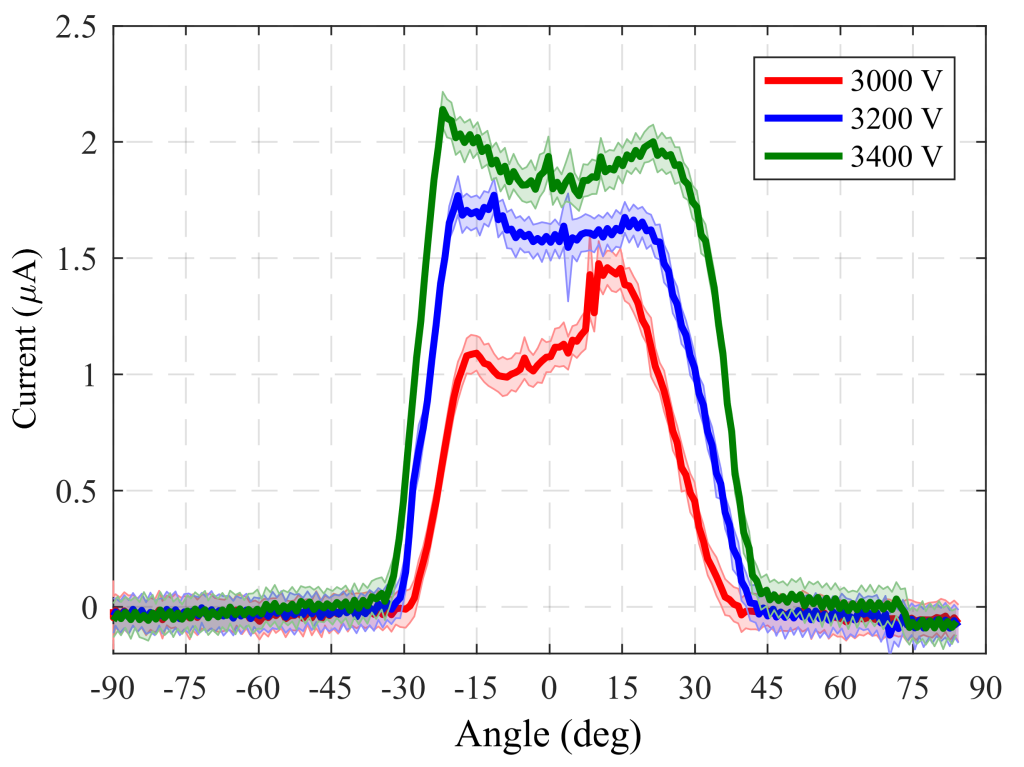

Figure D.3: Divergence map for EMIM-NTf2 ferrofluid emission with 2-magnets configuration (115 G). A 1-sigma standard deviation is shown with the shaded patch.

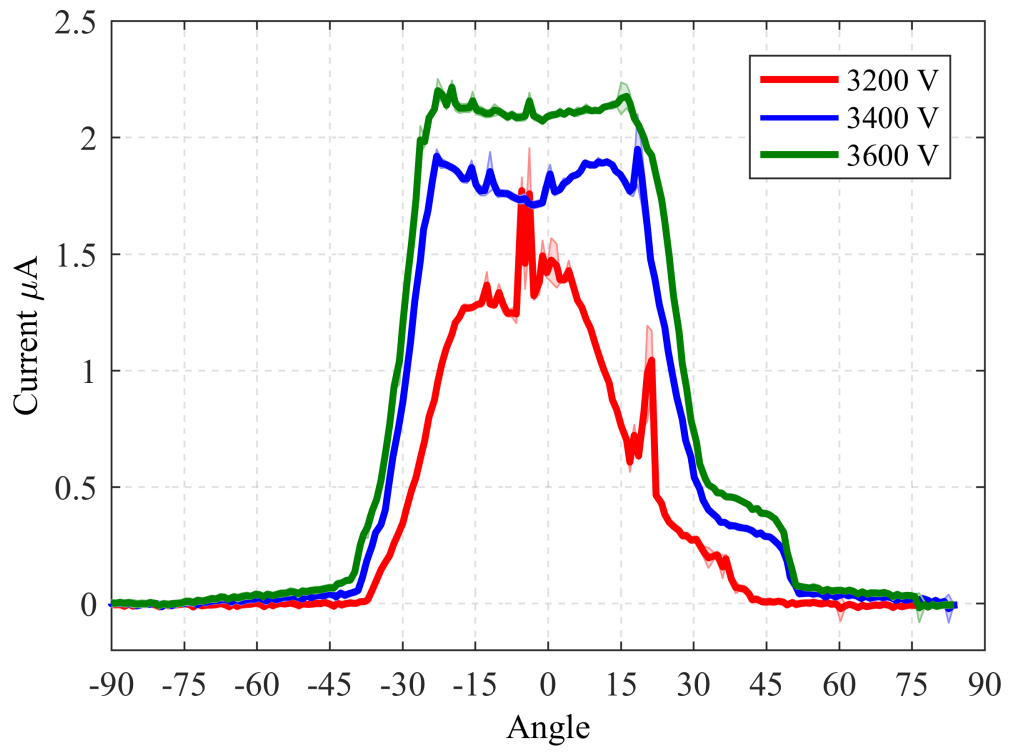

Figure D.4: Divergence map for EMIM-NTf2 ferrofluid emission with 3-magnets configuration ( $230 \mathrm{G})$.

The experimental approach employed in this study had excellent sensitivity for resolving the angular distribution considering a $0.9^{\circ}$ accuracy. However, this approach had several limitations which were undesirable for this application. They are as follows: 
1. The slow sweep rate was unable to attribute variations in the plume pattern to unsteady emission or variations in the plume.

2. This testing required a single polarity of emission be applied for the duration of the sweep. E.J Meyer observed a condition in which the fluid would experience bubbling, which likely due to electrochemistry and decomposition of the fluid - an observance not unique to ionic liquid ferrofluids. ${ }^{30}$ A Using a bipolar 0.5 $\mathrm{Hz}$ oscillation has been observed by researchers in the Ion Space Propulsion Laboratory at Michigan Technological University to resolve this for these fluids. The resulting sweep would need to be on the order of $90 \%$ s to resolve the distribution. 


\section{Appendix E Angular Power Efficiency Factor and Correlations Calculation Methodology}

\section{E1. Angular Power Efficiency Factor}

The angular power efficiency factor, $\eta_{\theta}$, was calculated during the duration of the

emission telemetry. The current density function, $\rho_{I}(\theta)$, was approximated by applying an interpolation function to the average measured emission current of a single polarity. Efficiencies were then averaged over a 1-minute interval to obtain a mean and standard deviation. Two methods of interpolating were investigated, a cubic spline and a Piecewise Cubic Hermite Interpolation Polynomial, or PCHIP. An example of these fits is presented below in Figure E.1. Overall, it was found the PCHIP method yielded more desirable results, owing to the lack of oscillations presented in the fit.

Fitting was performed using the spline and pchip functions in MATLAB which returned a piecewise polynomial structure, which was compatible with the pval and integrate functions to solve Eq. (6.4). A comparison of the efficiency obtained using the Spline and PCHIP integration methods is presented in Figure E.2. 

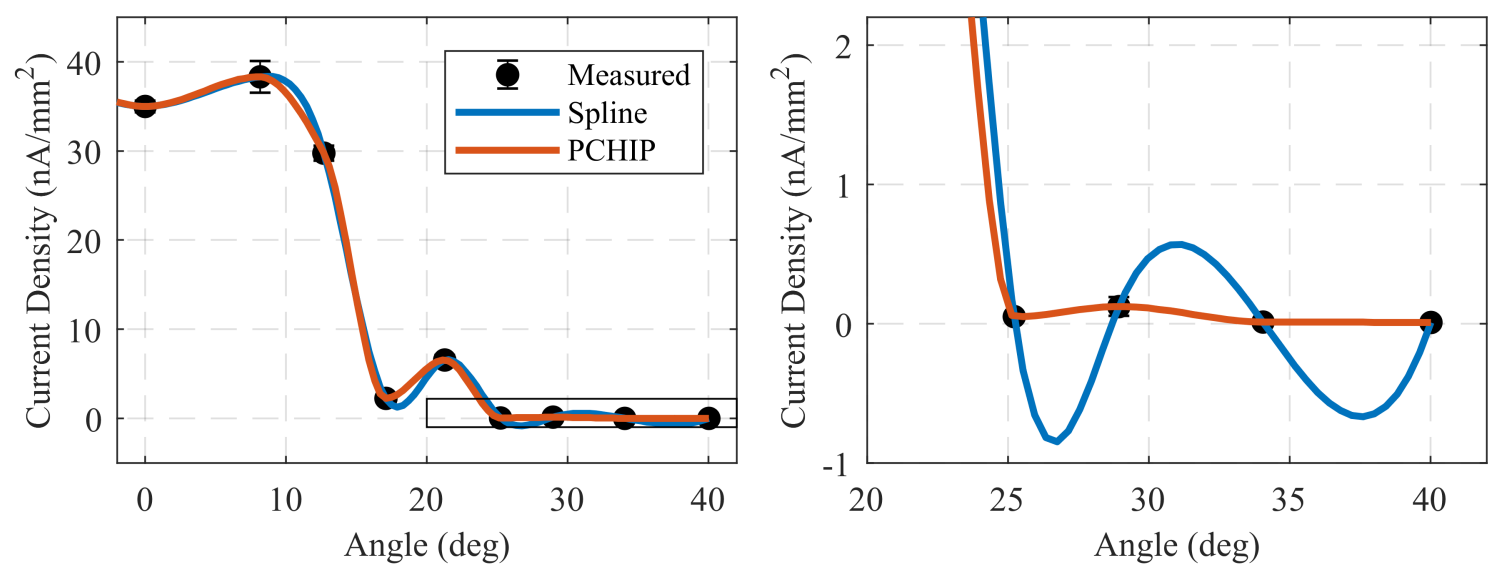

Figure E.1: Angular current density fitting using a cubic spline and a Piecewise Cubic Hermite Interpolation Polynomial (PCHIP) method. (Left) Full profile fit, (Right) Highlight of oscillations formed by spline interpolation between data points of similar density. Angular density was assumed to be symmetric about $0^{\circ}$.

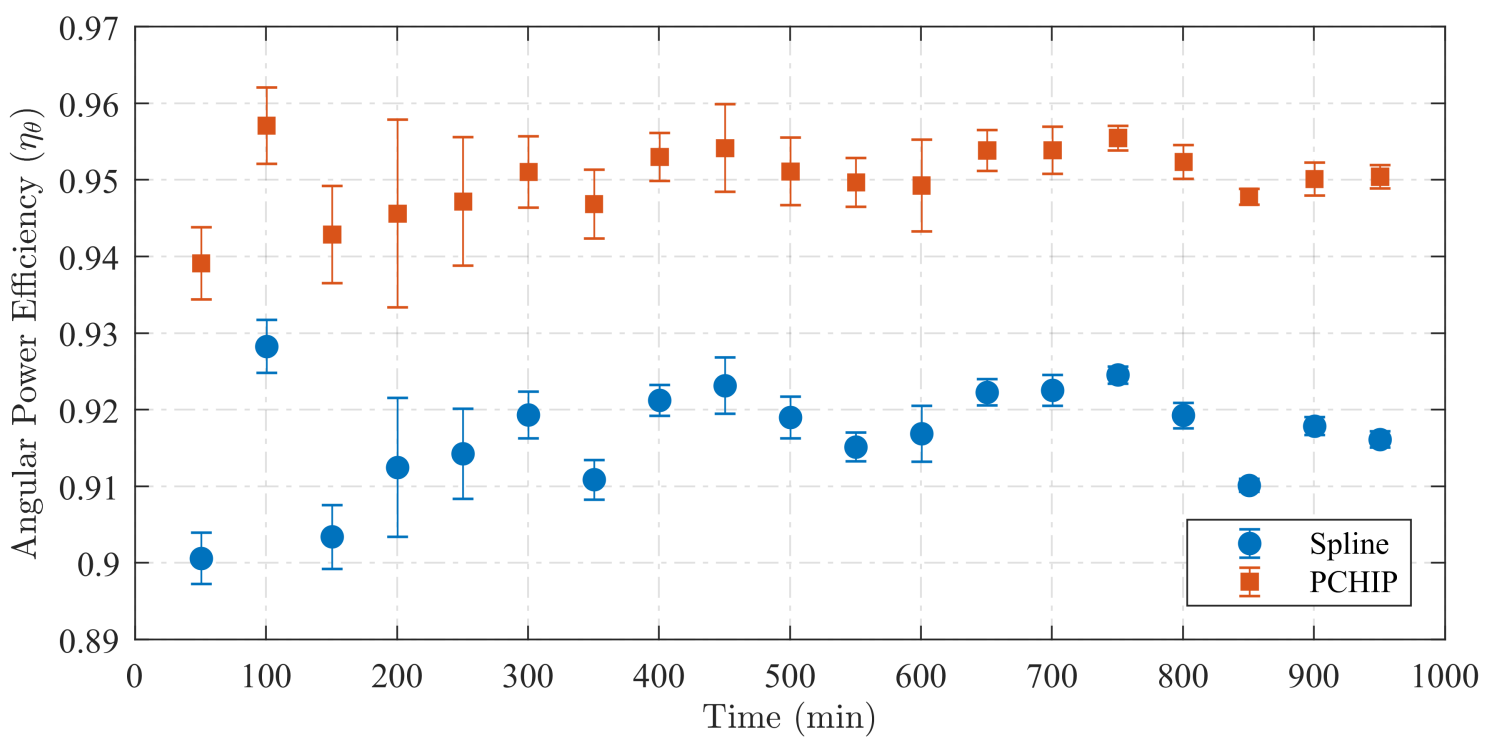

Figure E.2: Comparison in the angular efficiency calculated using the spline and PCHIP integration techniques.

While calculating the angular power efficiency, certain sample points within the telemetry had angular efficiencies which deviated significantly from the median for telemetry. These values were correlated with occurring during the startup and terminal phase of the telemetry. The angular efficiency with excluded sample points noted is presented in Figure E.3. 


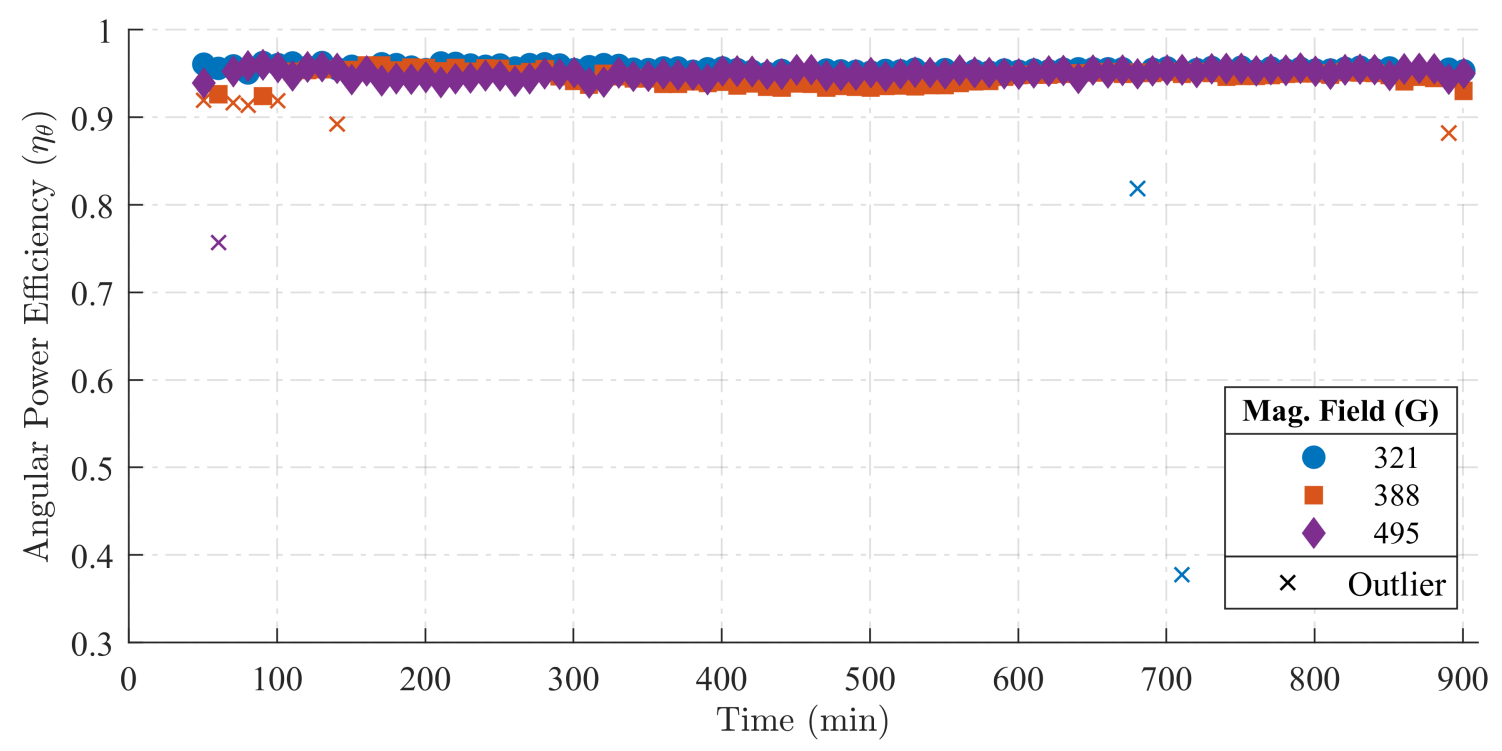

Figure E.3: Angular efficiency telemetry with outlying sample points noted.

\section{E2. Correlation Coefficients}

Correlation coefficients between mass flow rate, current, and angular efficiency were calculated using three correlation techniques. This analysis was performed using the corr function within MATLAB. Pearson, Kendall, and Spearman correlations were performed, where Pearson is a linear correlation and Kendall and Spearman are both rank order correlations. The results of these three analyses are presented in the tables below. Overall, the methods performed comparably well. The Spearman correlation was presented in Chapter 6, since the methodology does not assume a linear relation. 
Table E-1: Correlation coefficients obtained using the Pearson correlation method.

\begin{tabular}{|c|c|c|c|c|}
\hline & $\begin{array}{c}\text { B Fiel } \\
(\mathrm{G})\end{array}$ & & $\eta_{\theta}$ & $I$ \\
\hline \multirow{6}{*}{$\dot{m}$} & \multirow{2}{*}{321} & $\mathrm{R}$ & -0.562 & 0.480 \\
\hline & & $\mathrm{P}$ & $3.31 \mathrm{E}-08$ & $4.50 \mathrm{E}-06$ \\
\hline & \multirow{2}{*}{388} & $\mathrm{R}$ & -0.417 & 0.947 \\
\hline & & $\mathrm{P}$ & $1.18 \mathrm{E}-04$ & $3.77 \mathrm{E}-40$ \\
\hline & \multirow{2}{*}{495} & $\mathrm{R}$ & -0.327 & 0.795 \\
\hline & & $\mathrm{P}$ & $2.25 \mathrm{E}-03$ & $1.04 \mathrm{E}-19$ \\
\hline \multirow{6}{*}{$I$} & \multirow{2}{*}{321} & $\mathrm{R}$ & -0.806 & \multirow{2}{*}{$\mathrm{n}=83$} \\
\hline & & $\mathrm{P}$ & $4.15 \mathrm{E}-20$ & \\
\hline & \multirow{2}{*}{388} & $\mathrm{R}$ & -0.463 & \multirow{2}{*}{$\mathrm{n}=80$} \\
\hline & & $\mathrm{P}$ & $1.54 \mathrm{E}-05$ & \\
\hline & \multirow{2}{*}{495} & $\mathrm{R}$ & -0.482 & \multirow{2}{*}{$\mathrm{n}=85$} \\
\hline & & $\mathrm{P}$ & $3.00 \mathrm{E}-06$ & \\
\hline
\end{tabular}

Table E-2: Correlation coefficients obtained using the Kendall correlation method.

\begin{tabular}{|c|c|c|c|c|}
\hline & $\begin{array}{c}\text { B Fiel } \\
\text { (G) }\end{array}$ & & $\eta_{\theta}$ & $I$ \\
\hline \multirow{6}{*}{$\dot{m}$} & \multirow{2}{*}{321} & $\mathrm{R}$ & -0.412 & 0.365 \\
\hline & & $\mathrm{P}$ & $3.68 \mathrm{E}-08$ & $1.08 \mathrm{E}-06$ \\
\hline & \multirow{2}{*}{388} & $\mathrm{R}$ & -0.269 & 0.735 \\
\hline & & $\mathrm{P}$ & $4.20 \mathrm{E}-04$ & $4.85 \mathrm{E}-22$ \\
\hline & \multirow{2}{*}{495} & $\mathrm{R}$ & -0.241 & 0.575 \\
\hline & & $\mathrm{P}$ & $1.08 \mathrm{E}-03$ & $6.59 \mathrm{E}-15$ \\
\hline \multirow{6}{*}{$I$} & \multirow{2}{*}{321} & $\mathrm{R}$ & -0.646 & \multirow{2}{*}{$\mathrm{n}=83$} \\
\hline & & $\mathrm{P}$ & $5.42 \mathrm{E}-18$ & \\
\hline & \multirow{2}{*}{388} & $\mathrm{R}$ & -0.320 & \multirow{2}{*}{$\mathrm{n}=80$} \\
\hline & & $\mathrm{P}$ & $2.76 \mathrm{E}-05$ & \\
\hline & \multirow{2}{*}{495} & $\mathrm{R}$ & -0.377 & \multirow{2}{*}{$\mathrm{n}=85$} \\
\hline & & $\mathrm{P}$ & $3.31 \mathrm{E}-07$ & \\
\hline
\end{tabular}


Table E-3: Correlation coefficients obtained using the Spearman correlation method.

\begin{tabular}{|c|c|c|c|c|}
\hline \multicolumn{3}{|c|}{$\begin{array}{l}\text { B Field } \\
(\mathrm{G})\end{array}$} & $\eta_{\theta}$ & $I$ \\
\hline \multirow{6}{*}{$\dot{m}$} & \multirow{2}{*}{321} & $\mathrm{R}$ & -0.557 & 0.507 \\
\hline & & $\mathrm{P}$ & $8.07 \times 10^{-8}$ & $1.42 \times 10^{-6}$ \\
\hline & \multirow{2}{*}{388} & $\mathrm{R}$ & -0.383 & 0.893 \\
\hline & & $\mathrm{P}$ & $4.90 \times 10^{-4}$ & 0 \\
\hline & \multirow{2}{*}{495} & $\mathrm{R}$ & -0.355 & 0.774 \\
\hline & & $\mathrm{P}$ & $9.31 \times 10^{-4}$ & 0 \\
\hline \multirow{6}{*}{$I$} & \multirow{2}{*}{321} & $\mathrm{R}$ & -0.829 & \multirow{2}{*}{$\mathrm{n}=83$} \\
\hline & & $\mathrm{P}$ & 0 & \\
\hline & \multirow{2}{*}{388} & $\mathrm{R}$ & -0.457 & \multirow{2}{*}{$\mathrm{n}=80$} \\
\hline & & $\mathrm{P}$ & $2.47 \times 10^{-5}$ & \\
\hline & \multirow{2}{*}{495} & $\mathrm{R}$ & -0.506 & \multirow{2}{*}{$\mathrm{n}=85$} \\
\hline & & $\mathrm{P}$ & $1.15 \times 10^{-6}$ & \\
\hline
\end{tabular}





\section{References}

1. Krejci, D., Mier-Hicks, F., Thomas, R., Haag, T., and Lozano, P. "Emission Characteristics of Passively Fed Electrospray Microthrusters with Propellant Reservoirs," Journal of Spacecraft and Rockets, 2017, pp. 1-12.doi: $10.2514 / 1 . \mathrm{A} 33531$

2. Grustan-Gutierrez, E., and Gamero-Castaño, M. "Microfabricated Electrospray Thruster Array with High Hydraulic Resistance Channels," Journal of Propulsion and Power, 2017, pp. 1-8.doi: 10.2514/1.B36268

3. $\quad$ Jahn, R. G., and Choueiri, E. Y. "Electric Propulsion."

4. Johnson, W. "Contents and commentary on William Moore's A treatise on the motion of rockets and an essay on naval gunnery," International journal of impact engineering Vol. 16, No. 3, 1995, pp. 499-521.

5. Sutton, G. P., and Biblarz, O. Rocket Propulsion Elements. New Jersey: Wiley, 2010.

6. $\quad$ Pote, B., and Tedrake, R. "Performance of a high specific impulse Hall thruster," International Electric Propulsion Conference, IEPC-01-35, Pasadena, Ca. 2001.

7. Hofer, R. R., and Gallimore, A. D. "High-Specific Impulse Hall Thrusters, Part 2: Efficiency Analysis," Journal of Propulsion and Power Vol. 22, No. 4, 2006, pp. 732-740.

8. Hoskins, W. A., and Cassady, R. J. "30 years of electric propulsion flight experience at Aerojet Rocketdyne." 2013.

9. Foster, C., Hallam, H., and Mason, J. "Orbit determination and differential-drag control of planet labs cubesat constellations," arXiv preprint arXiv:1509.03270, 2015.

10. Specification, C. D. "CubeSat Design Rev. 12," Cal Poly, August, 2009.

11. Kidd, P. W. "Parametric studies with a single-needle colloid thruster," Journal of Spacecraft and Rockets Vol. 5, No. 9, 1968, pp. 1034-1039.doi: 10.2514/3.29417

12. Mahoney, J. F., Moore, R. D., Perel, J., and Yahiku, A. Y. "Research and development of a charged-particle bipolar thruster," AIAA Journal Vol. 7, No. 3, 1969, pp. 507-511.doi: $10.2514 / 3.5137$

13. Gamero-Castaño, M., and Hruby, V. "Electrospray as a Source of Nanoparticles for Efficient Colloid Thrusters," Journal of Propulsion and Power Vol. 17, No. 5, 2001, pp. 977-987.doi: 10.2514/2.5858 
14. Guerra-Garcia, C., Krejci, D., and Lozano, P. "Spatial uniformity of the current emitted by an array of passively fed electrospray porous emitters," Journal of Physics D: Applied Physics Vol. 49, No. 11, 2016, p. 115503.

15. Krpoun, R. "Micromachined electrospray thrusters for spacecraft propulsion," 2009.

16. Bier, M., and Dietrich, S. "Vapour pressure of ionic liquids," Molecular Physics Vol. 108, No. 2, 2010, pp. 211-214.

17. Galiński, M., Lewandowski, A., and Stępniak, I. "Ionic liquids as electrolytes," Electrochimica Acta Vol. 51, No. 26, 2006, pp. 5567-5580.doi: http://dx.doi.org/10.1016/j.electacta.2006.03.016

18. Romero-Sanz, I., Bocanegra, R., de la Mora, J. F., and Gamero-Castano, M. "Source of heavy molecular ions based on Taylor cones of ionic liquids operating in the pure ion evaporation regime," Journal of Applied Physics Vol. 94, No. 5, 2003, pp. 3599-3605.

19. Fröba, A. P., Kremer, H., and Leipertz, A. "Density, refractive index, interfacial tension, and viscosity of ionic liquids [EMIM][EtSO4],[EMIM][NTf2],[EMIM][N (CN) 2], and [OMA][NTf2] in dependence on temperature at atmospheric pressure," The Journal of Physical Chemistry B Vol. 112, No. 39, 2008, pp. 1242012430.

20. Williams, D. "Busek Electrospray Thrusters Launch aboard ESA's LISA Pathfinder," 2015.

21. Lozano, P., and Martinez-Sanchez, M. "Efficiency estimation of EMI-BF4 ionic liquid electrospray thrusters," 41st AIAA/ASME/SAE/ASEE Joint Propulsion Conference \& Exhibit. 2005, p. 4388.

22. Lemmer, K. "Propulsion for CubeSats," Acta Astronautica.doi: http://dx.doi.org/10.1016/j.actaastro.2017.01.048

23. Chakraborty, S., Courtney, D. G., and Shea, H. "A $10 \mathrm{nN}$ resolution thrust-stand for micro-propulsion devices," Review of Scientific Instruments Vol. 86, No. 11, 2015, p. 115109.

24. Hicks, F. M., Perna, L., Coffman, C. S., and Lozano, P. C. "Characterization of a CubeSat compatible magnetically levitated thrust balance for electrospray propulsion systems," 49th AIAA/ASME/SAE/ASEE Joint Propulsion Conference. American Institute of Aeronautics and Astronautics, 2013.

25. Courtney, D. G., Dandavino, S., and Shea, H. "Comparing Direct and Indirect Thrust Measurements from Passively Fed Ionic Electrospray Thrusters," Journal of Propulsion and Power, 2015.

26. Meyer IV, E. J., and King, L. B. "Electrospray from an Ionic Liquid Ferrofluid utilizing the Rosensweig Instability." 49th AIAA/ASME/SAE/ASEE Joint Propulsion Conferene \& Exhibit, Paper No. AIAA-2013-3823, 14-17 July 2013, San Jose, CA, 2013.

27. King, L. B., Jain, N., and Hawkett, B. S. "Ionic liquid ferrofluid electrospray with EMIM-NTf2 and ferrofluid mode studies with FerroTec EFH-1 in a non-uniform magnetic field." 2013. 
28. Patel, A. "Magnetically Levitating Low-Friction Test Stand for the Measurement of Micro-Thruster Performance Characteristics," 55th AIAA Aerospace Sciences Meeting. American Institute of Aeronautics and Astronautics, 2017.

29. Mkrtchyan, L., Zakinyan, A., and Dikansky, Y. "Electrocapillary instability of magnetic fluid peak," Langmuir Vol. 29, No. 29, 2013, pp. 9098-9103.

30. Meyer, E. J. "Development of an ionic liquid ferrofluid electrospray source and mode shape studies of a ferrofluid in a non-uniform magnetic field," Mechanical Engineering - Engineering Mechanics. Vol. Ph.D., Michigan Technological University, 2014.

31. Terhune, K. J. "Influence of Magnetic Nanoparticles and Magnetic Stress on an Ionic Liquid Electrospray Source," 2017.

32. Terhune, K. J., King, L. B., Hause, M. L., Prince, B. D., Jain, N., and Hawkett, B. S. "Species measurements in the beam of an ionic liquid ferrofluid electrospray source," 50th AIAA/ASME/SAE/ASEE Joint Propulsion Conference \& Exhibit. AIAA, Cleveland, OH, 2014.

33. Terhune, K. J., King, L. B., and Juillard, M. Y. "Influence of nanoparticles and magnetic surface stress on the emitted beam of an ionic liquid ferrofluid electrospray," 2017.

34. Terhune, K. J., King, L. B., Prince, B. D., Jain, N., and Hawkett, B. S. "The effects of magnetic surface stress on electrospray of an ionic liquid ferrofluid," 52nd AIAA/SAE/ASEE Joint Propulsion Conference. 2016, p. 4549.

35. Terhune, K. J., King, L. B., Prince, B. D., Jain, N., and Hawkett, B. S. "Species measurements in the beam of an ionic liquid ferrofluid capillary electrospray source under magnetic stress," 52nd AIAA/SAE/ASEE Joint Propulsion Conference. 2016, p. 4550.

36. Madden, A., Fernandez de la Mora, J., Jain, N., Sabouri, H., and Hawkett, B. "Effect of a homogeneous magnetic field on the electrospraying characteristics of sulfolane ferrofluids," Journal of Fluid Mechanics Vol. 833, 2017, pp. 430444.doi: $10.1017 / \mathrm{jfm} .2017 .589$

37. Taylor, G. "Disintegration of water drops in an electric field," Proceedings of the Royal Society of London A: Mathematical, Physical and Engineering Sciences. Vol. 280, The Royal Society, 1964, pp. 383-397.

38. Fernández de la Mora, J. "The Fluid Dynamics of Taylor Cones," Annual Review of Fluid Mechanics Vol. 39, No. 1, 2007, pp. 217-243.doi: 10.1146/annurev.fluid.39.050905.110159

39. Garoz, D., Bueno, C., Larriba, C., Castro, S., Romero-Sanz, I., de La Mora, J. F., Yoshida, Y., and Saito, G. "Taylor cones of ionic liquids from capillary tubes as sources of pure ions: The role of surface tension and electrical conductivity," Journal of Applied Physics Vol. 102, No. 6, 2007, p. 064913.

40. Gamero-Castaño, M. "Characterization of the electrosprays of 1-ethyl-3methylimidazolium bis (trifluoromethylsulfonyl) imide in vacuum," Physics of Fluids (1994-present) Vol. 20, No. 3, 2008, p. 032103.

41. Wang, Y., Tan, M. K., Go, D. B., and Chang, H.-C. "Electrospray cone-jet breakup and droplet production for electrolyte solutions," EPL (Europhysics Letters) Vol. 99, No. 6, 2012, p. 64003. 
42. Gamero-Castano, M., and Fernandez De La Mora, J. "Direct measurement of ion evaporation kinetics from electrified liquid surfaces," The Journal of Chemical Physics Vol. 113, No. 2, 2000, pp. 815-832.

43. Dumont, Q., and Cole, R. B. "Jean-Antoine Nollet: The father of experimental electrospray," Mass spectrometry reviews Vol. 33, No. 6, 2014, pp. 418-423.

44. Krause, C. A., Bill. "ORNL Review, State of the Laboratory." Vol. 29, 1995.

45. Zeleny, J. "The Electrical Discharge from Liquid Points, and a Hydrostatic Method of Measuring the Electric Intensity at Their Surfaces," Physical Review Vol. 3, No. 2, 1914, pp. 69-91.doi: 10.1103/PhysRev.3.69

46. Zeleny, J. "Instability of Electrified Liquid Surfaces," Physical Review Vol. 10, No. 1, 1917, pp. 1-6.

47. Melcher, J., and Taylor, G. "Electrohydrodynamics: a review of the role of interfacial shear stresses," Annual Review of Fluid Mechanics Vol. 1, No. 1, 1969, pp. 111-146.

48. Taylor, G. "Studies in Electrohydrodynamics. I. The Circulation Produced in a Drop by Electrical Field," Proceedings of the Royal Society of London. Series A, Mathematical and Physical Sciences Vol. 291, No. 1425, 1966, pp. 159-166.

49. "Press Release: The Nobel Prize in Chemistry 2002"." Nobel Media AB 2014, nobelprize.org.

50. Castellanos, A., and Sciences, I. C. f. M. Electrohydrodynamics: Springer, 1998.

51. Balanis, C. A. Advanced Engineering Electromagnetics: Wiley, 2012.

52. Wakai, C., Oleinikova, A., Ott, M., and Weingärtner, H. "How polar are ionic liquids? Determination of the static dielectric constant of an imidazolium-based ionic liquid by microwave dielectric spectroscopy," The Journal of Physical Chemistry B Vol. 109, No. 36, 2005, pp. 17028-17030.

53. De La Mora, J. F., and Loscertales, I. G. "The current emitted by highly conducting Taylor cones," Journal of Fluid Mechanics Vol. 260, 1994, pp. 155-184.

54. Dorf, R. C. CRC handbook of engineering tables: CRC Press, 2003.

55. Rosensweig, R. E. Ferrohydrodynamics: Courier Dover Publications, 1997.

56. Stephen, P. S. "Low viscosity magnetic fluid obtained by the colloidal suspension of magnetic particles." Google Patents, 1965.

57. Rosensweig, R., Hirota, Y., Tsuda, S., and Raj, K. "Study of audio speakers containing ferrofluid," Journal of Physics: Condensed Matter Vol. 20, No. 20, 2008, p. 204147.

58. Raj, K. "Ferrofluid rotary-shaft seal apparatus and method." Google Patents, 1982.

59. Hajec, C. S. "Electric motor with ferrofluid bearing." Google Patents, 1988.

60. Ruuge, E., and Rusetski, A. "Magnetic fluids as drug carriers: targeted transport of drugs by a magnetic field," Journal of Magnetism and Magnetic Materials Vol. 122, No. 1, 1993, pp. 335-339.

61. Maria, R. R., Simonetta Geninatti, C., Elisabetta, S., Paolo, S., Michele, F., Eleonora, C., Rachele, S., Fabrizio, D., and Silvio, A. "Magnetic hyperthermia efficiency and $1 \mathrm{H}-\mathrm{NMR}$ relaxation properties of iron oxide/paclitaxel-loaded PLGA nanoparticles," Nanotechnology Vol. 27, No. 28, 2016, p. 285104. 
62. Jain, N., Zhang, X., Hawkett, B. S., and Warr, G. G. "Stable and water-tolerant ionic liquid ferrofluids," ACS Applied Materials \& Interfaces Vol. 3, No. 3, 2011, pp. 662-667.

63. Huang, W., and Wang, X. "Study on the properties and stability of ionic liquidbased ferrofluids," Colloid and Polymer Science Vol. 290, No. 16, 2012, pp. 16951702.

64. Medeiros, A. M., Parize, A. L., Oliveira, V. M., Neto, B. A., Bakuzis, A. F., Sousa, M. H., Rossi, L. M., and Rubim, J. C. "Magnetic Ionic Liquids Produced by the Dispersion of Magnetic Nanoparticles in 1-n-Butyl-3-methylimidazolium bis (trifluoromethanesulfonyl) imide (BMI. NTf2)," ACS applied materials \& interfaces Vol. 4, No. 10, 2012, pp. 5458-5465.

65. Rodríguez-Arco, L., López-López, M. T., González-Caballero, F., and Durán, J. D. "Steric repulsion as a way to achieve the required stability for the preparation of ionic liquid-based ferrofluids," Journal of colloid and interface science Vol. 357, No. 1, 2011, pp. 252-254.

66. Smith, D. O. "Development of a Vibrating-Coil Magnetometer," Review of Scientific Instruments Vol. 27, No. 5, 1956, pp. 261-268.doi: 10.1063/1.1715538

67. Huke, B., and Lücke, M. "Magnetic properties of colloidal suspensions of interacting magnetic particles," Reports on Progress in Physics Vol. 67, No. 10, 2004, p. 1731.

68. Griffiths, D. J. Introduction to Electrodynamics: Prentice Hall, 1999.

69. Cowley, M., and Rosensweig, R. E. "The interfacial stability of a ferromagnetic fluid," Journal of Fluid mechanics Vol. 30, No. 04, 1967, pp. 671-688.

70. Boudouvis, A. G., Puchalla, J. L., Scriven, L. E., and Rosensweig, R. E. "Normal field instability and patterns in pools of ferrofluid," Journal of Magnetism and Magnetic Materials Vol. 65, No. 2, 1987, pp. 307-310.doi: https://doi.org/10.1016/0304-8853(87)90057-6

71. Abou, B., Wesfreid, J.-E., and Roux, S. "The normal field instability in ferrofluids: hexagon-square transition mechanism and wavenumber selection," Journal of Fluid Mechanics Vol. 416, 2000, pp. 217-237.doi: 10.1017/S002211200000882X

72. Zelazo, R. E., and Melcher, J. R. "Dynamics and stability of ferrofluids: surface interactions," Journal of Fluid Mechanics Vol. 39, No. 1, 2006, pp. 1-24.doi: 10.1017/S0022112069002011

73. Rosensweig, R. E. "Magnetic fluids," Annual review of fluid mechanics Vol. 19, No. 1, 1987, pp. 437-461.

74. Prewett, P. D., and Mair, G. L. R. Focused ion beams from liquid metal ion sources: Research Studies Press, 1991.

75. Krpoun, R., and Shea, H. R. "A method to determine the onset voltage of single and arrays of electrospray emitters," Journal of Applied Physics Vol. 104, No. 6, 2008, p. 064511.

76. Sherwood, J. "The deformation of a fluid drop in an electric field: a slender-body analysis," Journal of Physics A: Mathematical and General Vol. 24, No. 17, 1991, p. 4047.

77. Brancher, J., and Zouaoui, D. "Equilibrium of a magnetic liquid drop," Journal of magnetism and Magnetic Materials Vol. 65, No. 2, 1987, pp. 311-314. 
78. Stone, H. A., Lister, J. R., and Brenner, M. P. "Drops with conical ends in electric and magnetic fields," Proceedings of the Royal Society of London A: Mathematical, Physical and Engineering Sciences. Vol. 455, The Royal Society, 1999, pp. 329347.

79. Koromyslov, V., Grigor'ev, A., and Rybakova, M. "Disintegration of a drop in an external electrostatic field," Technical Physics Vol. 47, No. 6, 2002, pp. 682-689.

80. O'Konski, C. T., and Thacher Jr, H. C. "The distortion of aerosol droplets by an electric field," The Journal of Physical Chemistry Vol. 57, No. 9, 1953, pp. 955958.

81. Rowghanian, P., Meinhart, C. D., and Campàs, O. "Dynamics of ferrofluid drop deformations under spatially uniform magnetic fields," Journal of Fluid Mechanics Vol. 802, 2016, pp. 245-262.doi: 10.1017/jfm.2016.447

82. Tyatyushkin, A. N., and Velarde, M. G. "On the interfacial deformation of a magnetic liquid drop under the simultaneous action of electric and magnetic fields," Journal of colloid and interface science Vol. 235, No. 1, 2001, pp. 46-58.

83. Zakinyan, A., Tkacheva, E., and Dikansky, Y. "Dynamics of a dielectric droplet suspended in a magnetic fluid in electric and magnetic fields," Journal of Electrostatics Vol. 70, No. 2, 2012, pp. 225-232.

84. Gollwitzer, C., Spyropoulos, A., Papathanasiou, A., Boudouvis, A., and Richter, R. "The normal field instability under side-wall effects: comparison of experiments and computations," New Journal of Physics Vol. 11, No. 5, 2009, p. 053016.

85. Boudouvis, A., Puchalla, J., and Scriven, L. "Magnetohydrostatic equilibria of ferrofluid drops in external magnetic fields," Chemical Engineering Communications Vol. 67, No. 1, 1988, pp. 129-144.

86. Papathanasiou, A., and Boudouvis, A. "Three-dimensional instabilities of ferromagnetic liquid bridges," Computational mechanics Vol. 21, No. 4-5, 1998, pp. 403-408.

87. Reznik, S. N., Yarin, A. L., Theron, A., and Zussman, E. "Transient and steady shapes of droplets attached to a surface in a strong electric field," Journal of Fluid Mechanics Vol. 516, 2004, pp. 349-377.doi: 10.1017/s0022112004000679

88. Ramos, A., and Castellanos, A. "Equilibrium shapes and bifurcation of captive dielectric drops subjected to electric fields," Journal of electrostatics Vol. 33, No. 1, 1994, pp. 61-86.

89. Lavrova, O., Matthies, G., Mitkova, T., Polevikov, V., and Tobiska, L. "Numerical treatment of free surface problems in ferrohydrodynamics," Journal of Physics: Condensed Matter Vol. 18, No. 38, 2006, p. S2657.

90. Hawket, B., and Sabouri, H. "Saturatization Magnetization for ILFF Samples." 9/21/16, 2016.

91. King, L. B., Meyer, E., Hopkins, M. A., Hawkett, B. S., and Jain, N. "SelfAssembling Array of Magnetoelectrostatic Jets from the Surface of a Superparamagnetic Ionic Liquid," Langmuir Vol. 30, No. 47, 2014, pp. 1414314150.

92. Bryce, N. S., Pham, B. T., Fong, N. W., Jain, N., Pan, E. H., Whan, R. M., Hambley, T. W., and Hawkett, B. S. "The composition and end-group functionality of 
sterically stabilized nanoparticles enhances the effectiveness of co-administered cytotoxins," Biomaterials Science Vol. 1, No. 12, 2013, pp. 1260-1272.

93. Lozano, P., and Martínez-Sánchez, M. "Ionic liquid ion sources: suppression of electrochemical reactions using voltage alternation," Journal of Colloid and Interface Science Vol. 280, No. 1, 2004, pp. 149-154.doi: http://dx.doi.org/10.1016/j.jcis.2004.07.037

94. UltraVolt. "UltraVolt HVA Series Precision High voltage Amplifier." Vol. 2017, http://www.advanced-energy.com/upload/file/ultravolt/eng-hv-hvaseries-230h.pdf, 2017.

95. Jones, D. "uCurrent Original." Vol. 2017, 2010, p. https://www.eevblog.com/projects/ucurrentoriginal/.

96. Lozano, P., Martínez-Sánchez, M., and Lopez-Urdiales, J. M. "Electrospray emission from nonwetting flat dielectric surfaces," Journal of Colloid and Interface Science Vol. 276, No. 2, 2004, pp. 392-399.

97. Lenguito, G., de la Mora, J. F., and Gomez, A. "Scaling up the power of an electrospray microthruster," Journal of Micromechanics and Microengineering Vol. 24, No. 5, 2014, p. 055003.

98. Perez Martinez, C. S. "Engineering ionic liquid ion sources for ion beam applications." Massachusetts Institute of Technology, 2016.

99. Ryan, C., Stark, J. P., Ataman, C., Dandavino, S., Chakraborty, S., and Shea, H. "MicroThrust MEMS electrospray emitters-integrated microfabrication and test results," Space Propulsion 2012. Citeseer, 2012.

100. Lozano, P., Glass, B., and Martinez-Sanchez, M. "Performance characteristics of a linear ionic liquid electrospray thruster," 29th International Electric Propulsion Conference. Electric Rocket Propulsion Society Fairview Park, OH, 2005, pp. 2005-0192.

101. Lozano, P. C., Brikner, N. A., and Coffman, C. S. "Method and apparatus for a porous electrospray emitter." Google Patents, 2014.

102. Dandavino, S., Ataman, C., Ryan, C., Chakraborty, S., Courtney, D., Stark, J., and Shea, H. "Microfabricated electrospray emitter arrays with integrated extractor and accelerator electrodes for the propulsion of small spacecraft," Journal of Micromechanics and Microengineering Vol. 24, No. 7, 2014, p. 075011.

103. Miller, S. W. Analysis of ion emitting jet structures during ionic liquid electrospraying: Missouri University of Science and Technology, 2015.

104. Terhune, K. J., King, L. B., He, K., and Cumings, J. "Radiation-induced solidification of ionic liquid under extreme electric field," Nanotechnology Vol. 27, No. 37, 2016, p. 375701.

105. Jackson, B. A., King, L. B., Jain, N., and Hawkett, B. S. "Characterization of an Ionic Liquid Ferrofluid Electrospray Emission Pattern," 50th AIAA/ASME/SAE/ASEE Joint Propulsion Conference \& Exhibit. AIAA, Cleveland, $\mathrm{OH}, 2014$.

106. Gamero-Castano, M. "The structure of electrospray beams in vacuum," Journal of Fluid Mechanics Vol. 604, 2008, pp. 339-368. 
107. Morris, T., Malardier-Jugroot, C., and Jugroot, M. "Characterization of electrospray beams for micro-spacecraft electric propulsion applications," Journal of Electrostatics Vol. 71, No. 5, 2013, pp. 931-938.

108. Lozano, P., and Martínez-Sánchez, M. "Studies on the ion-droplet mixed regime in colloid thrusters," Department of Aeronautics and Astronautics, Massachusetts Institute of Technology, 2003.

109. Miller, S. W., Prince, B. D., Bemish, R. J., and Rovey, J. L. "Electrospray of 1Butyl-3-Methylimidazolium Dicyanamide Under Variable Flow Rate Operations," Journal of Propulsion and Power Vol. 30, No. 6, 2014, pp. 1701-1710.

110. Hofstadler, S. A., and Laude, D. A. "Electrospray ionization in the strong magnetic field of a Fourier transform ion cyclotron resonance mass spectrometer," Analytical Chemistry Vol. 64, No. 5, 1992, pp. 569-572.

111. Senko Michael, W., Hendrickson Christopher, L., Paša-Tolić, L., Marto Jarrod, A., White Forest, M., Guan, S., and Marshall Alan, G. "Electrospray Ionization Fourier Transform Ion Cyclotron Resonance at 9.4 T," Rapid Communications in Mass Spectrometry Vol. 10, No. 14, 1998, pp. 1824-1828.doi: 10.1002/(SICI)10970231(199611)10:14<1824::AID-RCM695>3.0.CO;2-E

112. Jaworek, A. "Electrospray droplet sources for thin film deposition," Journal of Materials Science Vol. 42, No. 1, 2007, pp. 266-297.

113. den Hartog, S. "Ionic liquid ferrofluid electrospray: a new propulsion for satellites," 2015.

114. Castellanos, A. Electrohydrodynamics: Springer Vienna, 2014. 